Supporting Information

\title{
1,2,4-Trifunctionalized Cyclohexane Synthesis via a Diastereoselective Reductive Cope Rearrangement and Functional Group Interconversion Strategy
}

\author{
Michael D. Mannchen, ${ }^{a}$ Ion Ghiviriga, ${ }^{b}$ Khalil A. Abboud, ${ }^{c}$ and Alexander J. Grenning ${ }^{a *}$

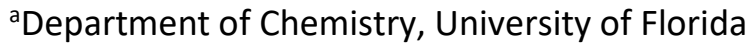 \\ ${ }^{b}$ Center for NMR Spectroscopy, Department of Chemistry, University of Florida \\ ${ }^{c}$ Center for X-ray Crystallography, Department of Chemistry, University of Florida \\ *Corresponding author, email: grenning@ufl.edu
}

\begin{abstract}
Polyfunctionalized cyclohexanes are privileged scaffolds in drug discovery. Reported herein is a method for synthesizing 1,2,4-trifunctionalized cyclohexanes via diastereoselective Cope rearrangement. The scaffolds obtained can be derivatized by orthogonal functional group interconversion to cyclohexanes bearing a 1-amide, 2-branched arylallyl, and variable 4-functional group
\end{abstract}


Table of Contents

General $\quad$ S-3

Experimental Procedures $\quad$ S-3

Electrophile synthesis $\quad$ S-3

Cyclohexylidene malononitrile synthesis $\quad$ S-3

6-aryl-3,3-dicyano-1,5 diene synthesis S-4

Reductive Cope Rearrangement $\quad$ S-4

Oxidative Amination S-5

$\begin{array}{lr}\text { Oxidative Esterification } & \text { S-6 }\end{array}$

$\begin{array}{lr}\text { Novel Molecules and Data } & \text { S-7 }\end{array}$

SI-1 to SI-4 S-7

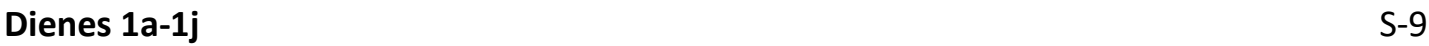

Alkylmalononitrile $3 f \quad S-15$

$\begin{array}{ll}\text { Amides 4a-4o } & \text { S-15 }\end{array}$

$\begin{array}{ll}\text { Molecules 5-7 } & \text { S-25 }\end{array}$

$\begin{array}{lr}\text { Molecules 8-10 S-29 } & \end{array}$

Stereochemical Assignment for malononitrile 3f $\quad$ S-31

$\begin{array}{ll}\text { Stereochemical Assignment for amide 4j } & \text { S-49 }\end{array}$

References $\quad$ S-54

NMR Spectra reprints $\quad$ S-55 


\section{General}

All commercial materials were used without further purification. ${ }^{1} \mathrm{H} N M R$ and ${ }^{13} \mathrm{C} N M R$ spectra were recorded in $\mathrm{CDCl}_{3}$, using a $400 \mathrm{MHz}, 500 \mathrm{MHz}$, or $600 \mathrm{MHz}$ spectrometer. All ${ }^{13} \mathrm{C} \mathrm{NMR}$ spectra were recorded with complete proton decoupling. HRMS data were recorded on Agilent Time of Flight 6230 spectrometer using ESI+ mode. Reaction progress was monitored by thin-layer chromatography (TLC) and visualized by UV light, phosphomolybdic acid stain, and $\mathrm{KMnO} 4$ stain. Commercially available anhydrous DMF (Arcos) stored over molecular sieves was used for $\alpha$-alkylation of Knoevenagel adducts, the reductive Cope rearrangement. Commercially available anhydrous $\mathrm{MeCN}$ was used for oxidative amidations and esterification. All other reactions (with the exception of Knoevenagel condensations) were carried out using anhydrous solvents obtained dried by passing through activated alumina columns (Pure Process Technology SPS). Unless otherwise noted, reactions were heated using preheated aluminum heating blocks from Pie-Blocks. Hantzsch ester was purchased from Oakwood and used without purification. Hantzsch ketone was synthesized from a known procedure. ${ }^{1}$

\section{Experimental Procedures}

\section{General procedure for all $[3,3]$ studies:}

1,5-diene 1a was dissolved in solvent (PhMe or DMF, $0.1 \mathrm{M}$ ) in a flame-dried pressure vial. The vials were then added to a preheated heating block and heated until the end of the time trial. The vials were then removed. If heated in toluene, solutions were concentrated under reduced pressure. If heated in DMF, the solvent was removed by a test tube workup with $2 \mathrm{~N}$ aq. $\mathrm{HCl} /$ ethyl acetate extraction. Conversions and diastereoselectivities were determined by ${ }^{1} \mathrm{H}$ NMR spectroscopy.

\section{Electrophile synthesis:}

Electrophiles E1-E4 were prepared according to literature procedures. ${ }^{2-7}$<smiles>Fc1ccc(/C=C/CBr)cc1</smiles>

E-1<smiles>COc1ccc(/C=C/CBr)cc1</smiles>

E-2<smiles>Fc1ccccc1/C=C/CBr</smiles>

E-3

\section{Cyclohexylidene malononitrile synthesis:}

The following cyclohexylidene malononitriles M1-M4 were prepared according to literature procedures. 8,9<smiles>N#CC(C#N)=C1CCC(c2ccccc2)CC1</smiles>

M1<smiles>CC1CCC(=C(C#N)C#N)CC1</smiles>

M2<smiles>CCOC(=O)C1CCC(=C(C#N)C#N)CC1</smiles>

M3<smiles>CC(C)NC1CCC(=C(C#N)C#N)CC1</smiles>

M4 
Procedure A.

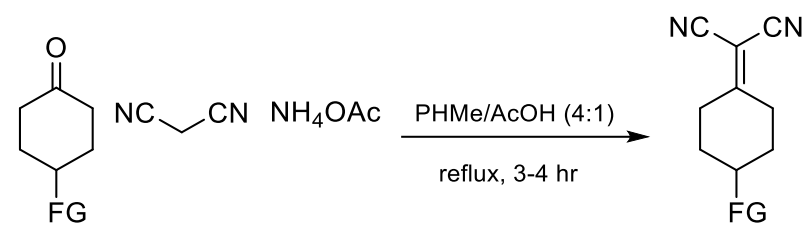

The following cyclohexylidene malononitriles are novel and were prepared by the following procedure: The cyclohexanone was dissolved in toluene $(1 \mathrm{M})$. Malononitrile (1.2 equiv.), ammonium acetate (1 equivalent), and glacial acetic acid $(4 \mathrm{M})$ were added. The reaction is heated at reflux $\left(120-140^{\circ} \mathrm{C}\right)$ with a Dean-Stark apparatus until complete by TLC. Reaction was the concentrated by rotary evaporation. Excess acetic acid was quenched with $2 \mathrm{~N}$ aq. $\mathrm{HCl}$. Aqueous layer was extracted with $3 \times 1 / 2$ volume with ethyl acetate. Combined organics were washed with brine, dried with sodium sulfate, and concentrated. Cyclohexylidene malononitrile was purified by recrystallization from ethanol.

\section{1,5-Diene Syntheses}<smiles>N#CC(C#N)=C1CCC(C(=O)O)CC1</smiles><smiles></smiles><smiles>CC(C#N)(C/C=C\[Al])C1=CCC(C(=O)O[Na])CC1</smiles>

Procedure B: Cyclohexlidene malononitrile $\mathrm{K}_{2} \mathrm{CO}_{3}$ (2 equiv.), and electrophile (1.5 equiv.) were dissolved in DMF (0.5M). Reactions were stirred at room temperature until starting material was consumed per TLC. Upon completion the reactions were quenched with $2 \mathrm{~N}$ aq. $\mathrm{HCl}$. The aqueous phase was extracted with EtOAc ( $2 \times 0.5$ volume). Combined organic layers were washed with brine, dried with $\mathrm{Na}_{2} \mathrm{SO}_{4}$ and concentrated in vacuo. Title compound was purified via flash column chromatography on silica gel.<smiles>C=CC(OC(C)(C)C)c1ccc(C2OCCO2)cc1</smiles>

Procedure C: To a flame-dried Schlenk flask under argon was added $\mathrm{Pd}\left(\mathrm{PPh}_{3}\right)_{4}(13.9 \mathrm{mg}, 12 \mu \mathrm{mol}, 5$ mol\%). The flask was evacuated and backfilled with $\mathrm{N}_{2} 3$ times. The electrophile $(73.5 \mathrm{mg}, 240 \mu \mathrm{mol}, 1$ equiv.) and $\mathrm{CH}_{2} \mathrm{Cl}_{2}(2.4 \mathrm{~mL}, 0.1 \mathrm{M})$ were added followed by cyclohexylidene malononitrile $\mathbf{S I - 1}$ (69.9 mg $240 \mu \mathrm{mol}, 1$ equiv.). The reaction was stirred at room temperature until complete per TLC. Upon completion the reactions were vacuum filtered over a silica plug and washed with ethyl acetate. Title compound was purified via flash column chromatography on silica gel. 


\section{Reductive Cope Rearrangement}

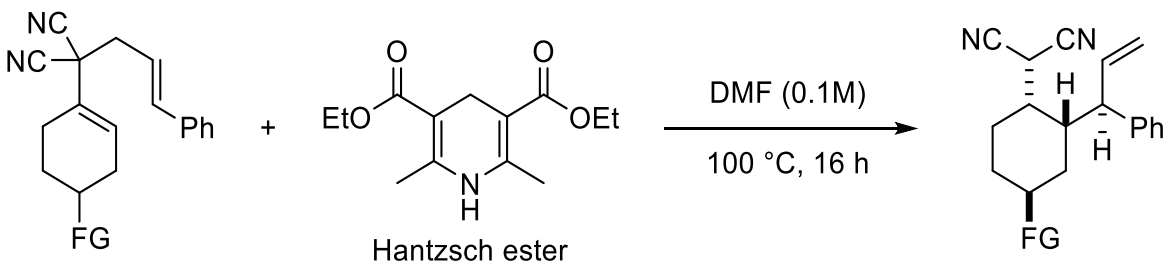

Procedure D: To a flame-dried Schlenk flask was added 1,5-diene and Hantzsch ester (10 equivalents). A septum was affixed and the flask was vacuum degassed and backfilled with $\mathrm{N}_{2}$ three times. Anhydrous DMF was added and the flask was placed in a preheated aluminum block and stirred at $100{ }^{\circ} \mathrm{C}$ for the 16 h. Upon completion, 30 equiv. of $\mathrm{LiOH}(2 \mathrm{~N}$ in $\mathrm{EtOH})$ solution was added and the mixture was stirred at $80^{\circ} \mathrm{C}$ for $30 \mathrm{~min}$. The reaction turned from bright yellow to orange. Reaction was then cooled in an ice bath and subsequently vacuum filtrated to remove excess Hantzsch ester and byproducts. A work-up was performed by first washing twice with $2 \mathrm{~N} \mathrm{NaHCO}_{3}$ (aq.) and the combined aqueous layers were back extracted twice with ethyl acetate. Combined organics were washed with water, dried with anhydrous $\mathrm{Na}_{2} \mathrm{SO}_{4}$ and concentrated by rotary evaporation. Flash column chromatography was performed. Purified malononitriles were then used in the oxidative amidations.

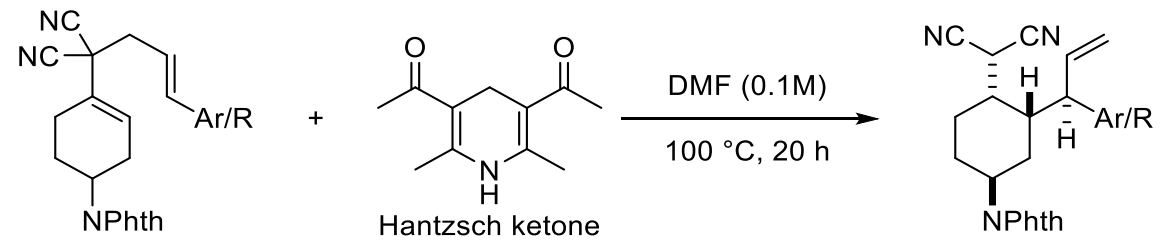

Procedure E: To a flame-dried Schlenk flask was added 1,5-diene $(0.2 \mathrm{mmol})$ and Hantzsch methyl ketone (10 or 20 equiv.). A septum was affixed and the flask was vacuum degassed and backfilled with $\mathrm{N}_{2}$ three times. DMF ( $2 \mathrm{~mL}, 0.1 \mathrm{M}$ ) was added and the flask was placed in a preheated aluminum block and stirred at $100^{\circ} \mathrm{C}$ for the $1 \mathrm{~h}$. Reaction was then cooled in an ice bath and subsequently vacuum filtrated to remove excess Hantzsch ester and byproducts. A work-up was performed by first washing twice with $2 \mathrm{~N} \mathrm{HCl}$ (aq.) and the combined aqueous layers were back extracted twice with ethyl acetate. Combined organics were washed with water, dried with anhydrous $\mathrm{Na}_{2} \mathrm{SO}_{4}$ and concentrated by rotary evaporation. Flash column chromatography was performed in most cases with an ethyl acetate/hexanes solvent system. Purified malononitriles were then used in the oxidative amidations. 

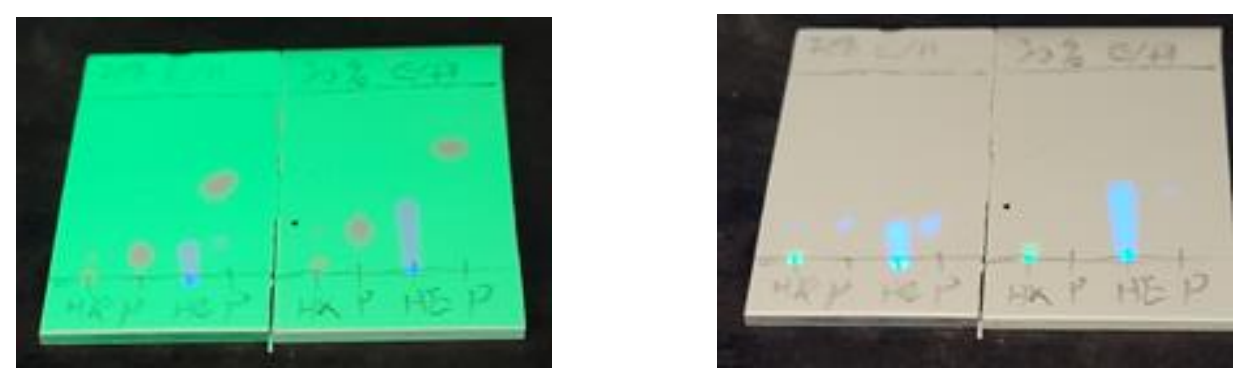

$20 \%$ ethyl acetate $30 \%$ ethyl acetate in hexanes

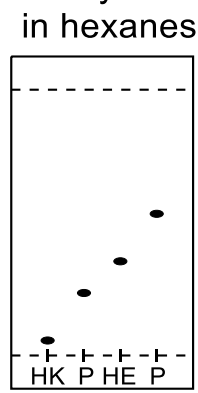

Figure S1. TLC plates comparing Rf of reductant and pyridines under long- and short-wave UV light. In $20 \%$ ethyl acetate/hexanes (left) and $30 \%$ ethyl acetate/hexanes (right). HK = Hantzsch ketone, $\mathrm{HE}=$ Hantzsch ester. $\mathrm{P}=$ pyridine byproduct. (Photos taken by Michael Mannchen)

\section{Oxidative amidation}<smiles>C=CC(Br)C1CC(C(F)F)CCC1C(C)C#N</smiles><smiles></smiles>

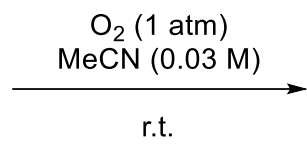

r.t.<smiles>[R16]C(=O)C1CCC([O-])CC1C([Al])C=C</smiles>

Procedure F: The malononitrile $(0.2 \mathrm{mmol})$ was dissolved in acetonitrile $(0.03 \mathrm{M})$. The amine (5 equiv.) and $\mathrm{K}_{2} \mathrm{CO}_{3}$ (5 equiv.) were added. An oxygen balloon was bubbled through the reaction mixture and the reaction was stirred at room temperature until complete by TLC. Once complete, the reaction mixture was vacuum filtered through a silica plug and washed with ethyl acetate. Crude mixture was purified using column chromatography.<smiles>C=CC(Br)C1CC(C(F)F)CCC1C(C#N)C#N</smiles>

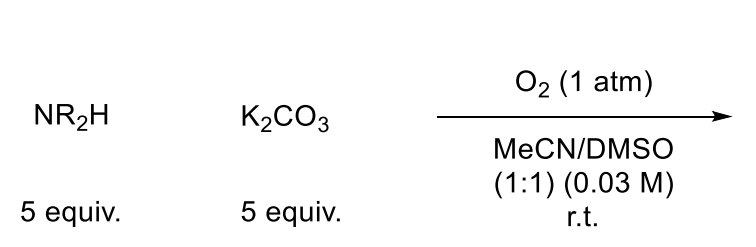

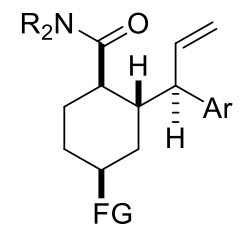

Procedure G: The malononitrile was dissolved in acetonitrile/DMSO (3:1) (0.03M). The amine (5 equiv.) and $\mathrm{K}_{2} \mathrm{CO}_{3}$ (5 equiv.) were added. An oxygen balloon was bubbled through the reaction mixture and the reaction was stirred at room temperature until complete by TLC. Once complete, the reaction mixture was vacuum filtered through a silica plug and washed with ethyl acetate. DMSO was removed by washing with $5 x$ volume of $2 \mathrm{~N}$ aq. $\mathrm{HCl}$ and back extracting with ethyl acetate. Combined organics were washed with brine, dried with $\mathrm{Na}_{2} \mathrm{SO}_{4}$, and concentrated. Crude mixture was purified using column chromatography. 


\section{Novel molecules and data}<smiles>N#CC(C#N)=C1CCC([PbH])CC1</smiles>

2-(4-(1,3-dioxoisoindolin-2-yl)cyclohexylidene)malononitrile (SI-1)

Prepared using Procedure A from the corresponding ketone (10 g, $41.28 \mathrm{mmol})$

Isolated: $9.86 \mathrm{~g}$;

Yield: 82\%;

Appearance: Light brown solid;

TLC: $\mathrm{Rf}=0.24$ in $30 \%$ ethyl acetate/hexane

${ }^{1} \mathrm{H}$ NMR $\left(600 \mathrm{MHz}, \mathrm{CDCl}_{3}\right) \delta 7.84(\mathrm{dd}, J=5.4,3.0 \mathrm{~Hz}, 2 \mathrm{H}), 7.74(\mathrm{dd}, J=5.5,3.0 \mathrm{~Hz}, 2 \mathrm{H}), 4.50-4.41(\mathrm{~m}$, $1 \mathrm{H}), 3.27-3.16(\mathrm{~m}, 2 \mathrm{H}), 2.56-2.44(\mathrm{~m}, 5 \mathrm{H}), 2.09(\mathrm{ddt}, J=9.0,3.5,1.5 \mathrm{~Hz}, 2 \mathrm{H}) .{ }^{13} \mathrm{C} \mathrm{NMR}\left(151 \mathrm{MHz}, \mathrm{CDCl}_{3}\right)$ $\delta 180.54,168.05,134.40,131.81,123.56,111.47,84.68,48.05,33.30,29.32$.

${ }^{13} \mathrm{C}$ NMR $\left(151 \mathrm{MHz}, \mathrm{CDCl}_{3}\right) \delta 180.5,168.1,134.4,131.8,123.6,111.5,84.7,48.1,33.3,29.3$.

HRMS: (ESI-TOF): Calculated for $\mathrm{m} / \mathrm{z}:[\mathrm{M}+\mathrm{H}]^{+}=292.1081$. Found 292.1086

Novel Molecules and Data<smiles>CC(C)C1CCC(=C(C#N)C#N)CC1</smiles>

2-(4-isopropylcyclohexylidene)malononitrile (SI-2)

Prepared using Procedure A from the corresponding ketone $(1 \mathrm{~g}, 7.13 \mathrm{mmol})$

Isolated: $1.19 \mathrm{~g}$; Yield: 89\%;

Appearance: Light yellow oil;

TLC: $\mathrm{Rf}=0.53$ in $20 \%$ ethyl acetate/hexanes.

${ }^{1} \mathrm{H}$ NMR $\left(600 \mathrm{MHz}, \mathrm{CDCl}_{3}\right) \delta 3.09-3.02(\mathrm{~m}, 2 \mathrm{H}), 2.31$ (ddd, $\left.J=14.3,12.7,5.2 \mathrm{~Hz}, 2 \mathrm{H}\right), 2.06$ (ddt, $J=13.4$, 5.4, $2.8 \mathrm{~Hz}, 2 \mathrm{H}), 1.58-1.47(\mathrm{~m}, J=6.7 \mathrm{~Hz}, 1 \mathrm{H}), 1.41$ (dddt, $J=12.2,9.1,6.1,3.2 \mathrm{~Hz}, 1 \mathrm{H}), 1.28(q d, J=$ $12.9,3.7 \mathrm{~Hz}, 2 \mathrm{H}), 0.90(\mathrm{~d}, J=6.8 \mathrm{~Hz}, 6 \mathrm{H})$.

${ }^{13} \mathrm{C}$ NMR $(151 \mathrm{MHz}, \mathrm{CDCl} 3) \delta$ 185.2, 111.8, 82.6, 42.8, 34.5, 31.9, 30.9, 19.9. 
HRMS: (ESI-TOF): $\mathrm{m} / \mathrm{z}:[\mathrm{M}-\mathrm{H}]$ ]: Calculated for $\mathrm{C}_{12} \mathrm{H}_{15} \mathrm{~N}_{2}=187.1241 \mathrm{~m} / \mathrm{z}$. Found $187.1248 \mathrm{~m} / \mathrm{z}$.
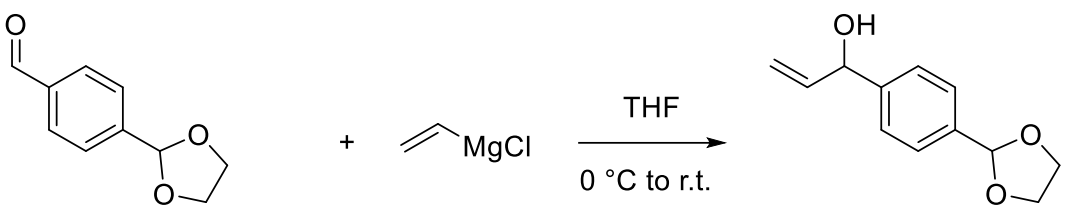

1-(4-(1,3-dioxolan-2-yl)phenyl)prop-2-en-1-ol (SI-3)

To a flame dried flask under nitogen was added aldehyde ${ }^{11}$ (480 mg, $2.69 \mathrm{mmol}$ ). Anhydrous THF (27 mL) was added and the reaction was cooled to $0{ }^{\circ} \mathrm{C}$. Vinylmagnesium chloride $(2.52 \mathrm{~mL}, 1.6 \mathrm{M}$ in THF, 1.5 equiv.) was added dropwise and the reaction was allowed to slowly warm to room temperature. Upon completion by $\mathrm{TLC}$, the reaction was quenched with saturated aqueous $\mathrm{NH}_{4} \mathrm{Cl}$. The aqueus layer was extracted with ethyl acetate $(2 \times 25 \mathrm{~mL})$. The combined organic layer was washed with brine, dried with $\mathrm{Na}_{2} \mathrm{SO}_{4}$, and concentrated in vacuo. The reaction mixture was purified by flash column chromatography on silica gel. (20-30\% ethyl acetate in hexanes.

Yield: $392 \mathrm{mg}$

Isolated: $70 \%$

Appearance: colorless oil

TLC: $\mathrm{Rf}=0.35$ in $30 \%$ ethyl acetate/hexanes

${ }^{1}$ H NMR $\left(600 \mathrm{MHz}, \mathrm{CDCl}_{3}\right) \delta 7.48-7.45(\mathrm{~m}, 2 \mathrm{H}), 7.38(\mathrm{dd}, J=8.3,1.9 \mathrm{~Hz}, 2 \mathrm{H}), 5.81(\mathrm{~s}, 1 \mathrm{H}), 5.33(\mathrm{dt}, J=$ $17.2,1.5 \mathrm{~Hz}, 1 \mathrm{H}), 5.21(\mathrm{~s}, 1 \mathrm{H}), 5.19(\mathrm{dt}, J=10.3,1.5 \mathrm{~Hz}, 1 \mathrm{H}), 4.16-4.07(\mathrm{~m}, 2 \mathrm{H}), 4.07-3.99(\mathrm{~m}, 3 \mathrm{H})$.

${ }^{13} \mathrm{C}$ NMR $\left(151 \mathrm{MHz}, \mathrm{CDCl}_{3}\right) \delta 143.8,140.2,137.5,126.8,115.4,103.6,75.2,65.4$.

HRMS: m/z: $[\mathrm{M}-\mathrm{H}]^{+}$Calculated for $\mathrm{C}_{12} \mathrm{H}_{13} \mathrm{O}_{3}=205.0865$. Found 205.0841 .<smiles>C=CC(O)c1ccc(C2OCCO2)cc1</smiles>

1-(4-(1,3-dioxolan-2-yl)phenyl)allyl tert-butyl carbonate (SI-4)

To a flame dried flask under nitrogen was added alcohol (SI-3) (199.6 mg, $0.968 \mathrm{mmol}$ ) dissolved in THF $(4.8 \mathrm{~mL}, 0.2 \mathrm{M})$. The reaction mixture was cooled to $0{ }^{\circ} \mathrm{C}$. ${ }^{n} \mathrm{BuLi}(1.06 \mathrm{~mL}, 1.06 \mathrm{mmol}, 1.1$ equiv. $1.6 \mathrm{M}$ in hexane) was added dropwise and the reaction was allowed to stir for 10 minutes. $\mathrm{Boc}_{2} \mathrm{O}(.0245 \mathrm{~mL}, 1.06$ mmol, 1.1 equiv.) was added and the reaction was allowed to stir until completion by TLC. The reaciton was quenched with saturated aqueous $\mathrm{NH}_{4} \mathrm{Cl}$. The aqueous layer was extracted with ethyl acetate $(2 \times 25$ $\mathrm{mL}$ ) and the combined organics were washed with brine, dried with $\mathrm{Na}_{2} \mathrm{SO}_{4}$, and concentrated. The reaction was purified via flash column chromatography on silica gel (10\% ethyl acetate in hexanes).

Yield: $87 \%$

Isolated: $257 \mathrm{mg}$ 
Appearance: colorless oil

TLC: $\mathrm{Rf}=0.65$ in $30 \%$ ethyl acetate in hexanes

${ }^{1}$ H NMR $\left(600 \mathrm{MHz}, \mathrm{CDCl}_{3}\right) \delta 7.51-7.48(\mathrm{~m}, 2 \mathrm{H}), 7.42-7.40(\mathrm{~m}, 2 \mathrm{H}), 6.06-6.04(\mathrm{~m}, 2 \mathrm{H}), 6.02(\mathrm{dd}, J=$ $10.2,6.1 \mathrm{~Hz}, 1 \mathrm{H}), 5.83(\mathrm{~s}, 1 \mathrm{H}), 5.37-5.29(\mathrm{~m}, 1 \mathrm{H}), 5.29-5.24(\mathrm{~m}, 1 \mathrm{H}), 4.17-4.11(\mathrm{~m}, 2 \mathrm{H}), 4.10-4.03$ $(\mathrm{m}, 2 \mathrm{H}), 1.49(\mathrm{~s}, 9 \mathrm{H})$.

${ }^{13} \mathrm{CNMR}\left(151 \mathrm{MHz}, \mathrm{CDCl}_{3}\right) \delta 152.7,139.8,137.9,136.1,127.0,126.7,117.4,103.5,82.4,78.9265 .3$, $65.3,27.8$.

HRMS: (ESI-TOF) m/z: [M+H] $]^{+}$Calculated for $\mathrm{C}_{17} \mathrm{H}_{23} \mathrm{O}_{5}=307.1540$, found 307.1548.<smiles>N#CC(C#N)(CC/C=C\c1ccccc1)C1=CCC(c2ccccc2)CC1</smiles>

2-cinnamyl-2-(1,2,3,6-tetrahydro-[1,1'-biphenyl]-4-yl)malononitrile (1a)

Prepared using General Procedure B from N1 (705 mg, 3.17 mmol) and cinnamyl bromide

Isolated: $943 \mathrm{mg}$;

Yield: $88 \%$ yield;

Physical state: white solid; TLC: $R_{f}=0.48$ in $20 \%$ Ethyl acetate in hexanes, purified with column chromatography 0-5\% ethyl acetate hexanes.

${ }^{1} \mathrm{H}$ NMR $\left(600 \mathrm{MHz}, \mathrm{CDCl}_{3}\right) \delta 7.43(\mathrm{~d}, J=7.2 \mathrm{~Hz}, 2 \mathrm{H}), 7.37(\mathrm{t}, J=7.5 \mathrm{~Hz}, 2 \mathrm{H}), 7.35-7.30(\mathrm{~m}, 3 \mathrm{H}), 7.28-$ $7.24(\mathrm{~m}, 1 \mathrm{H}), 7.22(\mathrm{~d}, J=7.7 \mathrm{~Hz}, 2 \mathrm{H}), 6.71(\mathrm{~d}, J=15.7 \mathrm{~Hz}, 1 \mathrm{H}), 6.37(\mathrm{dt}, J=4.8,2.1 \mathrm{~Hz}, 1 \mathrm{H}), 6.18(\mathrm{dt}, J=$ 15.3, 7.4 Hz, 1H), $2.98(\mathrm{q}, J=6.5 \mathrm{~Hz}, 2 \mathrm{H}), 2.85$ (tdt, $J=8.5,5.1,2.8 \mathrm{~Hz}, 1 \mathrm{H}), 2.53(\mathrm{dt}, J=18.3,5.4 \mathrm{~Hz}, 1 \mathrm{H}$ ), $2.41-2.35(\mathrm{~m}, 2 \mathrm{H}), 2.35-2.27(\mathrm{~m}, 1 \mathrm{H}), 2.17-2.10(\mathrm{~m}, 1 \mathrm{H}), 1.89(\mathrm{tdd}, J=12.5,9.8,5.9 \mathrm{~Hz}, 1 \mathrm{H})$.

${ }^{13} \mathrm{C}$ NMR: ${ }^{13} \mathrm{C}$ NMR $\left(151 \mathrm{MHz}, \mathrm{CDCl}_{3}\right) \delta 145,137.5,135.8,129.5,128.6,128 ., 128.3,127.6,126.6,126.61$, $126.5,119.2,114.3,114.1,43.8,40.8,38.7,32.9,29.2,25.0$.

HRMS: (ESI-TOF) m/z: $[\mathrm{M}+\mathrm{H}]^{+}$Calculated fo $\mathrm{C}_{24} \mathrm{H}_{23} \mathrm{~N}_{2}=339.1856$ Found $=339.1873$.

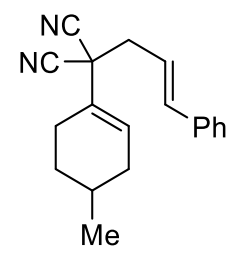

2-cinnamyl-2-(4-methylcyclohex-1-en-1-yl)malononitrile (1b)

Prepared using General Procedure B from N2 (250 mg, $1.56 \mathrm{mmol})$ and cinnamyl bromide Isolated: $348 \mathrm{mg}$ 
Yield: $81 \%$

Physical state: yellow/white solid.

TLC: $R_{f} 0.61$ in $20 \%$ ethyl acetate/hexanes. Column $5 \%$ ethyl acetate hexanes

${ }^{1}$ H NMR: $\left(600 \mathrm{MHz}, \mathrm{CDCl}_{3}\right) \delta 7.42(\mathrm{~d}, J=7.2 \mathrm{~Hz}, 2 \mathrm{H}), 7.37(\mathrm{t}, J=7.5 \mathrm{~Hz}, 2 \mathrm{H}), 7.35-7.30(\mathrm{~m}, 1 \mathrm{H}), 6.69(\mathrm{~d}, J$ $=15.7 \mathrm{~Hz}, 1 \mathrm{H}), 6.24(\mathrm{dt}, J=4.7,2.3 \mathrm{~Hz}, 1 \mathrm{H}), 6.16(\mathrm{dt}, J=15.3,7.4 \mathrm{~Hz}, 1 \mathrm{H}), 3.00-2.90(\mathrm{~m}, 2 \mathrm{H}), 2.34-2.20$ $(\mathrm{m}, 3 \mathrm{H}), 1.92-1.85(\mathrm{~m}, 1 \mathrm{H}), 1.80(\mathrm{ddt}, J=11.5,5.7,3.0 \mathrm{~Hz}, 1 \mathrm{H}), 1.75-1.66(\mathrm{~m}, 1 \mathrm{H}), 1.36(\mathrm{dtd}, J=12.9$, $10.3,5.9 \mathrm{~Hz}, 1 \mathrm{H}), 1.02(\mathrm{~d}, J=6.6 \mathrm{~Hz}, 3 \mathrm{H})$.

${ }^{13} \mathrm{C}$ NMR $\left(151 \mathrm{MHz}, \mathrm{CDCl}_{3}\right) \delta 137.6,136.0,129.7,128.8,128.5,127.4,126.8,119.5,114.7,114.4,44.0$, $41.1,33.7,30.4,27.6,24.7,21.2$.

HRMS: (ESI-TOF) m/z: $[\mathrm{M}+\mathrm{H}]^{+}$Calculated for $\mathrm{C}_{19} \mathrm{H}_{21} \mathrm{~N}_{2}=277.1699$, Found $=277.1696$

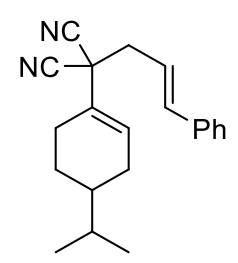

2-cinnamyl-2-(4-isopropylcyclohex-1-en-1-yl)malononitrile (1c)

Prepared using Procedure B from SI-2 (300 mg, $1.59 \mathrm{mmol})$ and cinnamyl bromide

Isolated: $427.8 \mathrm{mg}$

Yield: $88 \%$

Physical state: colorless oil

TLC: $\mathrm{Rf}=0.68$ in $20 \%$ ethyl acetate/hexanes. Column: $5 \%$ ethyl acetate/ hexanes

${ }^{1} \mathrm{H}$ NMR: ${ }^{1} \mathrm{H}$ NMR $\left(600 \mathrm{MHz}, \mathrm{CDCl}_{3}\right) \delta 7.43-7.38(\mathrm{~m}, 2 \mathrm{H}), 7.35(\mathrm{~d}, J=7.4 \mathrm{~Hz}, 2 \mathrm{H}), 7.33-7.27(\mathrm{~m}, 1 \mathrm{H})$, $6.67(\mathrm{~d}, J=15.7 \mathrm{~Hz}, 1 \mathrm{H}), 6.24(\mathrm{dt}, J=5.1,2.3 \mathrm{~Hz}, 1 \mathrm{H}), 6.15$ (dt, $J=15.3,7.4 \mathrm{~Hz}, 1 \mathrm{H}$ ), 2.92 (qdd, $J=13.9$, 7.5, $1.3 \mathrm{~Hz}, 2 \mathrm{H}), 2.34-2.20(\mathrm{~m}, 2 \mathrm{H}), 1.94(\mathrm{ddt}, J=10.0,4.6,2.3 \mathrm{~Hz}, 1 \mathrm{H}), 1.92-1.84(\mathrm{~m}, 1 \mathrm{H}), 1.52(\mathrm{dq}, J=$ $13.2,6.6 \mathrm{~Hz}, 1 \mathrm{H}), 1.36-1.30(\mathrm{~m}, 2 \mathrm{H}), 0.92(\mathrm{dd}, J=8.7,6.7 \mathrm{~Hz}, 6 \mathrm{H})$.

${ }^{13} \mathrm{C}$ NMR $\left(151 \mathrm{MHz}, \mathrm{CDCl}_{3}\right)$ $\delta 137.6,136.0,130.1,128.8,128.5,127.6,126.8,119.5,114.7,114.4,43.9$, 41.0, 39.3, 31.9, 29.1, 25.9, 25.4, 19.9, 19.7.

HRMS: (ESI-TOF): Calculated for m/z: $[\mathrm{M}-\mathrm{H}]^{-} \mathrm{C}_{21} \mathrm{H}_{23} \mathrm{~N}_{2}=303.1867$. Found 303.1871.<smiles>CCOC(=O)C1CC=C(C(C#N)(C#N)C/C=C\c2ccccc2)CC1</smiles> 
ethyl (E)-4-(1,1-dicyano-4-phenylbut-3-en-1-yl)cyclohex-3-ene-1-carboxylate (1d)

Prepared by General Procedure B from N3 (500 mg, 2.29 mmol) and cinnamyl bromide

Isolated: $702 \mathrm{mg}$

Yield: $92 \%$

Physical state: white solid

TLC: $R_{f}=0.32$ in $20 \%$ ethyl acetate in hexanes. Column $15 \%$ ethyl acetate/hexanes

${ }^{1}$ H NMR: $\left(600 \mathrm{MHz}, \mathrm{CDCl}_{3}\right) \delta 7.43-7.36(\mathrm{~m}, 2 \mathrm{H}), 7.34(\mathrm{dd}, J=8.5,6.7 \mathrm{~Hz}, 2 \mathrm{H}), 7.30-7.26(\mathrm{~m}, 1 \mathrm{H}), 6.66$ $(\mathrm{d}, J=15.7 \mathrm{~Hz}, 1 \mathrm{H}), 6.24(\mathrm{tt}, J=3.7,1.5 \mathrm{~Hz}, 1 \mathrm{H}), 6.12(\mathrm{dt}, J=15.3,7.4 \mathrm{~Hz}, 1 \mathrm{H}), 4.14(\mathrm{qq}, J=7.4,3.7 \mathrm{~Hz}$, $2 \mathrm{H}), 2.96-2.87(\mathrm{~m}, 2 \mathrm{H}), 2.61-2.54(\mathrm{~m}, 1 \mathrm{H}), 2.46-2.40(\mathrm{~m}, 2 \mathrm{H}), 2.37-2.27(\mathrm{~m}, 1 \mathrm{H}), 2.25(\mathrm{ddq}, J=14.7$, 8.9, $2.8 \mathrm{~Hz}, 1 \mathrm{H}$ ), 2.13 (dtd, $J=13.3,5.1,3.3 \mathrm{~Hz}, 1 \mathrm{H}$ ), 1.84 (dddd, $J=13.2,10.4,9.2,5.6 \mathrm{~Hz}, 1 \mathrm{H}), 1.26(\mathrm{t}, J=$ $7.1 \mathrm{~Hz}, 3 \mathrm{H})$.

${ }^{13}$ C NMR: $\left(151 \mathrm{MHz}, \mathrm{CDCl}_{3}\right) \delta$ 174.3, 137.6, 135.8, 128.7, 128.4, 128.1, 127.4, 126.7, 119.1, 114.2, 114.08, $60.7,43.7,40.8,37.9,27.4,24.7,23.7,14.2$.

HRMS: $\mathrm{m} / \mathrm{z}:[\mathrm{M}+\mathrm{H}]^{+}$Calculated for $\mathrm{C}_{21} \mathrm{H}_{23} \mathrm{~N}_{2} \mathrm{O}_{2}=335.1754$ Found $=335.1769$.

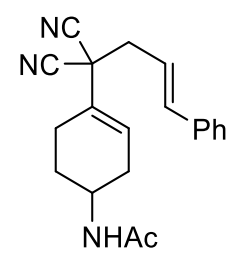

(E)-N-(4-(1,1-dicyano-4-phenylbut-3-en-1-yl)cyclohex-3-en-1-yl)acetamide (1e)

Prepared using General Procedure B from N4 (500 mg, 2.47 mmol) and cinnamyl bromide

Isolated: $569 \mathrm{mg}$

Yield: $72 \%$

Physical state: white solid

TLC: $\mathrm{Rf}=0.24$ in $2.5 \% \mathrm{MeOH} / \mathrm{DCM}$, Column: $0-2.5 \% \mathrm{MeOH}$ in DCM

${ }^{1}$ H NMR: $\left(600 \mathrm{MHz}, \mathrm{CDCl}_{3}\right) \delta 7.34(\mathrm{t}, J=7.6 \mathrm{~Hz}, 2 \mathrm{H}), 7.31-7.26(\mathrm{~m}, 1 \mathrm{H}), 6.67(\mathrm{~d}, J=15.7 \mathrm{~Hz}, 1 \mathrm{H}), 6.20$ (ddt, $J=4.8,3.2,1.4 \mathrm{~Hz}, 1 \mathrm{H}), 6.12(\mathrm{dt}, J=15.3,7.4 \mathrm{~Hz}, 1 \mathrm{H}), 5.37$ (d, $J=7.8 \mathrm{~Hz}, 1 \mathrm{H}$ ), 4.11 (dtdd, $J=10.8$, 8.1, 5.5, 3.1 Hz, 1H), $3.02-2.87(\mathrm{~m}, 2 \mathrm{H}), 2.62-2.54(\mathrm{~m}, 1 \mathrm{H}), 2.33(\mathrm{ddd}, J=9.7,6.2,3.2 \mathrm{~Hz}, 2 \mathrm{H}), 2.08-$ $1.99(\mathrm{~m}, 2 \mathrm{H}), 1.92(\mathrm{~s}, 3 \mathrm{H}), 1.70$ (dddd, $J=12.8,9.8,8.3,5.9 \mathrm{~Hz}, 1 \mathrm{H}$ ).

${ }^{13} \mathrm{C}$ NMR: $\left(151 \mathrm{MHz}, \mathrm{CDCl}_{3}\right) \delta 169.8,137.8,135.8,128.9,128.7,128.0,127.7,126.8,119.1,114.3,114.1$, $43.8,43.8,41.1,31.8,27.8,23.3$.

HRMS: (ESI-TOF) m/z: m/z: [M+H] ${ }^{+}$Calculated for $\mathrm{C}_{20} \mathrm{H}_{22} \mathrm{~N}_{3} \mathrm{O}: 320.1757$. Found 320.1760 . 
<smiles>N#CC(C#N)(CC/C=C\c1ccccc1)C1=CCC([NH2+]c2ccccc2)CC1</smiles>

2-cinnamyl-2-(4-(1,3-dioxoisoindolin-2-yl)cyclohex-1-en-1-yl)malononitrile (1f)

Prepared using General Procedure B from SI-1 (3.49 g, $12 \mathrm{mmol})$ and cinnamyl bromide (2.84 g, 14.4 mmol, 1.2 equiv.), with $\mathrm{K}_{2} \mathrm{CO}_{3}(2.49 \mathrm{~g}, 18 \mathrm{mmol}, 1.5$ equiv.)

Isolated: $4.15 \mathrm{~g}$

Yield: $92 \%$

Physical state: light yellow solid

TLC: $\mathrm{Rf}=0.41$ in $30 \%$ ethyl acetate/hexanes, column $20-30 \%$ ethyl acetate hexanes

${ }^{1} \mathbf{H}$ NMR $\left(600 \mathrm{MHz}, \mathrm{CDCl}_{3}\right) \delta 7.89-7.82(\mathrm{~m}, 2 \mathrm{H}), 7.78-7.70(\mathrm{~m}, 2 \mathrm{H}), 6.74(\mathrm{~d}, J=15.7 \mathrm{~Hz}, 1 \mathrm{H}), 6.33-6.27$ $(\mathrm{m}, 1 \mathrm{H}), 6.20(\mathrm{dt}, J=15.3,7.4 \mathrm{~Hz}, 1 \mathrm{H}), 4.44$ (dddd, $J=12.6,10.7,5.7,3.2 \mathrm{~Hz}, 1 \mathrm{H}), 3.06$ (ddq, $J=17.0$, 10.9, $3.0 \mathrm{~Hz}, 1 \mathrm{H}$ ), 2.97 (qdd, $J=13.8,7.4,1.3 \mathrm{~Hz}, 2 \mathrm{H}$ ), 2.65 (tdd, $J=12.6,9.8,7.2 \mathrm{~Hz}, 1 \mathrm{H}$ ), 2.47 (ddd, $J=$ 8.0, 3.9, $2.2 \mathrm{~Hz}, 2 \mathrm{H}), 2.44-2.35(\mathrm{~m}, 1 \mathrm{H}), 2.06-1.99(\mathrm{~m}, 1 \mathrm{H})$.

${ }^{13}$ C NMR: $\left(151 \mathrm{MHz}, \mathrm{CDCl}_{3}\right) \delta 168.2,137.9,135.8,134.2,131.9,128.8,128.5,128.3,127.7,126.8,123.3$, $119.1,114.3,114.0,46.1,43.82,41.2,28.5,26.0,25.3$.

HRMS: (ESI-TOF) m/z: [M+H] $]^{+}$Calculated for $\mathrm{C}_{26} \mathrm{H}_{22} \mathrm{~N}_{3} \mathrm{O}_{2}=408.1707$. Found 408.1704.

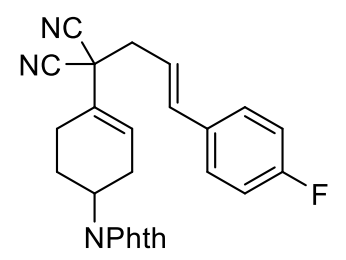

(E)-2-(4-(1,3-dioxoisoindolin-2-yl)cyclohex-1-en-1-yl)-2-(3-(4-fluorophenyl)allyl)malononitrile (1g)

Prepared using General Procedure B from SI-1 $(230 \mathrm{mg}, 0.79 \mathrm{mmol})$ and E-1

Isolated: $145 \mathrm{mg}$

Yield: $43 \%$

Physical state: light yellow solid

TLC: $\mathrm{Rf}=0.5$ in $30 \%$ ethyl acetate/hexanes

${ }^{1} \mathrm{H}$ NMR $\left(600 \mathrm{MHz}, \mathrm{CDCl}_{3}\right) \delta 7.86(\mathrm{td}, J=5.7,2.7 \mathrm{~Hz}, 2 \mathrm{H}), 7.75(\mathrm{td}, J=6.6,6.0,2.8 \mathrm{~Hz}, 2 \mathrm{H}), 7.43$ (ddd, $J=$ 8.6, 5.6, 3.0 Hz, 2H), $7.06(\mathrm{t}, J=8.6 \mathrm{~Hz}, 2 \mathrm{H}), 6.70(\mathrm{~d}, J=15.7 \mathrm{~Hz}, 1 \mathrm{H}), 6.31-6.27(\mathrm{~m}, 1 \mathrm{H}), 6.12(\mathrm{dt}, J=$ 15.3, $7.5 \mathrm{~Hz}, 1 \mathrm{H}$ ), 4.44 (dddd, $J=13.3,10.7,5.7,3.3 \mathrm{~Hz}, 1 \mathrm{H}$ ), 3.06 (tdt, $J=11.1,5.9,3.2 \mathrm{~Hz}, 1 \mathrm{H}$ ), 2.97 (qd, $J=13.9,7.4 \mathrm{~Hz}, 2 \mathrm{H}), 2.66(\mathrm{tdd}, J=12.6,9.7,7.3 \mathrm{~Hz}, 1 \mathrm{H}), 2.47(\mathrm{dd}, J=7.5,3.8 \mathrm{~Hz}, 2 \mathrm{H}), 2.45-2.38(\mathrm{~m}, 1 \mathrm{H})$, $2.04-1.99(\mathrm{~m}, 1 \mathrm{H})$. 
${ }^{13} \mathrm{C}$ NMR $\left(151 \mathrm{MHz}, \mathrm{CDCl}_{3}\right) \delta 168.2,162.8(\mathrm{~d}, J=247.8 \mathrm{~Hz}), 136.7,134.2,131.8,127.6,123.3,118.8$, $118.8,115.8,114.2,113.9,46.0,43.8,41.1,25.9,25.3$.

${ }^{19} \mathrm{~F}$ NMR $\left(565 \mathrm{MHz}, \mathrm{CDCl}_{3}\right) \delta-112.93$.

HRMS: (ESI-TOF) m/z: [M+H] ${ }^{+}$Calculated for $\mathrm{C}_{26} \mathrm{H}_{21} \mathrm{FN}_{3} \mathrm{O}_{2}$ : 426.1612. Found: 426.1624.<smiles>N#CC(C#N)(C/C=C/c1ccccc1F)C1=CCC(Nc2ccccc2)CC1</smiles>

(S,E)-2-(4-(1,3-dioxoisoindolin-2-yl)cyclohex-1-en-1-yl)-2-(3-(2-fluorophenyl)allyl)malononitrile (1h) Prepared using General Procedure B from SI-1 (291.3 mg, $1 \mathrm{mmol})$ and E-3

Isolated: $237 \mathrm{mg}$

Yield: $56 \%$

Physical state: yellow solid

TLC: $\mathrm{Rf}=0.46$ in $30 \%$ ethyl acetate/hexanes

${ }^{1} \mathbf{H}$ NMR $\left(600 \mathrm{MHz}, \mathrm{CDCl}_{3}\right) \delta 7.83(\mathrm{td}, J=6.9,6.2,3.1 \mathrm{~Hz}, 3 \mathrm{H}), 7.79-7.64(\mathrm{~m}, 3 \mathrm{H}), 7.48(\mathrm{td}, J=7.7,1.7 \mathrm{~Hz}$, $1 \mathrm{H}), 7.26(\mathrm{td}, J=10.6,9.0,4.1 \mathrm{~Hz}, 1 \mathrm{H}), 7.13(\mathrm{t}, J=7.5 \mathrm{~Hz}, 1 \mathrm{H}), 7.05(\mathrm{dd}, J=10.7,8.3 \mathrm{~Hz}, 1 \mathrm{H}), 6.85(\mathrm{~d}, J=$ $15.9 \mathrm{~Hz}, 1 \mathrm{H}), 6.26(\mathrm{p}, J=7.6 \mathrm{~Hz}, 2 \mathrm{H}), 4.41$ (dddd, $J=13.4,10.8,5.7,3.2 \mathrm{~Hz}, 1 \mathrm{H}), 3.00$ (dqd, $J=28.3,13.9$, $9.1 \mathrm{~Hz}, 3 \mathrm{H}), 2.81-2.70(\mathrm{~m}, \mathrm{OH}), 2.63(\mathrm{tdd}, J=12.6,9.7,7.3 \mathrm{~Hz}, 1 \mathrm{H}), 2.54-2.48(\mathrm{~m}, \mathrm{OH}), 2.48-2.35(\mathrm{~m}$, $3 \mathrm{H}), 2.27-2.20(\mathrm{~m}, \mathrm{OH}), 2.04-1.97(\mathrm{~m}, 1 \mathrm{H})$.

${ }^{13} \mathrm{C}$ NMR (151 MHz, CDCl 3 ) $\delta$ 168.32, 160.44 (d, $\left.J=250.4 \mathrm{~Hz}\right), 134.3,131.9,130.5$ (d, $\left.J=3.3 \mathrm{~Hz}\right), 130.0$ (d, $J=8.6 \mathrm{~Hz}$ ), 128.6, 128.0, 127.9, 127.7, 124.4 (d, $J=3.7 \mathrm{~Hz}), 123.4,122.0$ (d, $J=5.1 \mathrm{~Hz}$ ), 116.1, 115.9, $114.3,114.0,46.2,43.8,41.5,28.6,26.0,25.4$.

${ }^{19} \mathrm{~F}$ NMR $\left(565 \mathrm{MHz} \mathrm{CDCl}_{3}\right) \delta-117.31$.

HRMS: (ESI-TOF) m/z: [M+Na] ${ }^{+}$Calculated for $\mathrm{C}_{26} \mathrm{H}_{20} \mathrm{FNaN}_{3} \mathrm{O}_{2}=448.1432$. Found 448.1428.

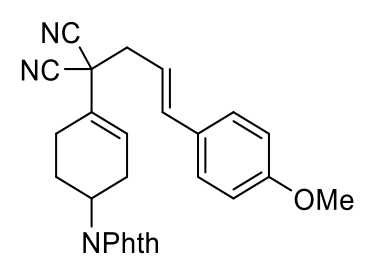

(S,E)-2-(4-(1,3-dioxoisoindolin-2-yl)cyclohex-1-en-1-yl)-2-(3-(4-methoxyphenyl)allyl)malononitrile (1i) Prepared using General Procedure B from SI-1 (134 mg, $0.46 \mathrm{mmol}$ ) and E-2

Isolated: $149 \mathrm{mg}$ 
Yield: $74 \%$

Physical state: light yellow solid

TLC: 0.43 in $40 \%$ ethyl acetate/hexanes.

${ }^{1} \mathbf{H}$ NMR $\left(600 \mathrm{MHz}, \mathrm{CDCl}_{3}\right) \delta 7.87-7.78(\mathrm{~m}, 2 \mathrm{H}), 7.76-7.67(\mathrm{~m}, 2 \mathrm{H}), 7.40-7.34(\mathrm{~m}, 2 \mathrm{H}), 6.91-6.84(\mathrm{~m}$, $2 \mathrm{H}), 6.65(\mathrm{~d}, J=15.6 \mathrm{~Hz}, 1 \mathrm{H}), 6.25(\mathrm{dt}, J=5.3,1.9 \mathrm{~Hz}, 1 \mathrm{H}), 6.03(\mathrm{dt}, J=15.3,7.4 \mathrm{~Hz}, 1 \mathrm{H}), 4.45-4.37(\mathrm{~m}$, $1 \mathrm{H}$ ), $3.82(\mathrm{~s}, 3 \mathrm{H}$ ), 3.03 (tdd, $J=13.4,5.8,2.9 \mathrm{~Hz}, 1 \mathrm{H}$ ), 2.93 (qdd, $J=13.8,7.5,1.2 \mathrm{~Hz}, 2 \mathrm{H}$ ), 2.63 (tdd, $J=$ 12.6, 9.9, $7.1 \mathrm{~Hz}, 1 \mathrm{H}), 2.44(\mathrm{dp}, J=6.8,4.1,3.0 \mathrm{~Hz}, 2 \mathrm{H}), 2.41-2.32(\mathrm{~m}, 1 \mathrm{H}), 2.04-1.96(\mathrm{~m}, 1 \mathrm{H})$.

${ }^{13} \mathrm{C}$ NMR $\left(151 \mathrm{MHz}, \mathrm{CDCl}_{3}\right) \delta 168.2,159.9,137.4,134.2,131.8,128.6,128.1,127.7,123.3,116.6,114.13$, $55.4,46.1,43.9,41.3,28.4,25.9,25.3$.

HRMS: (ESI-TOF) m/z: [M+H] $]^{+}$Calculated for $\mathrm{C}_{27} \mathrm{H}_{24} \mathrm{~N}_{3} \mathrm{O}_{3}=438.1812$ Found $=438.1835$.

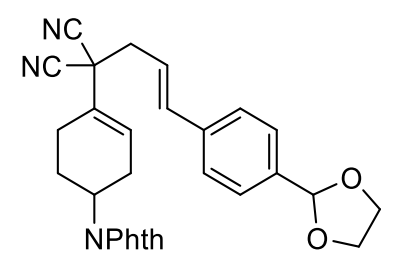

(E)-2-(3-(4-(1,3-dioxolan-2-yl)phenyl)allyl)-2-(4-(1,3-dioxoisoindolin-2-yl)cyclohex-1-en-1-yl)malononitrile (1j)

Synthesized using General Procedure C from SI-1 and SI-4

Yield: $83 \%$

Isolated: $95 \mathrm{mg}$

Appearance: yellow solid

TLC: $\mathrm{Rf}=0.28$ in $30 \%$ ethyl acetate/hexanes

${ }^{1} \mathrm{H}$ NMR $\left(600 \mathrm{MHz}, \mathrm{CDCl}_{3}\right) \delta 7.83(\mathrm{dd}, J=5.4,3.1 \mathrm{~Hz}, 2 \mathrm{H}), 7.72(\mathrm{dd}, J=5.5,3.0 \mathrm{~Hz}, 2 \mathrm{H}), 7.46(\mathrm{~d}, J=8.4 \mathrm{~Hz}$, $2 \mathrm{H}), 7.43(\mathrm{~d}, J=8.4 \mathrm{~Hz}, 2 \mathrm{H}), 6.71(\mathrm{~d}, J=15.7 \mathrm{~Hz}, 1 \mathrm{H}), 6.28-6.23(\mathrm{~m}, 1 \mathrm{H}), 6.19(\mathrm{dt}, J=15.4,7.5 \mathrm{~Hz}, 1 \mathrm{H})$, $5.81(\mathrm{~s}, 1 \mathrm{H}), 4.40$ (dddd, $J=12.6,10.7,5.6,3.2 \mathrm{~Hz}, 1 \mathrm{H}), 4.15-4.08(\mathrm{~m}, 2 \mathrm{H}), 4.06-3.99(\mathrm{~m}, 2 \mathrm{H}), 3.03$ (ddq, $J=16.7,10.9,3.0 \mathrm{~Hz}, 1 \mathrm{H}$ ), 2.94 (qdd, $J=13.8,7.4,1.3 \mathrm{~Hz}, 2 \mathrm{H}$ ), 2.62 (tdd, $J=12.6,9.6,7.3 \mathrm{~Hz}, 1 \mathrm{H}$ ), 2.44 (ddd, $J=8.4,4.3,2.3 \mathrm{~Hz}, 2 \mathrm{H}$ ), 2.38 (dtd, $J=17.6,5.5,1.5 \mathrm{~Hz}, 1 \mathrm{H}), 2.01-1.96(\mathrm{~m}, 1 \mathrm{H})$.

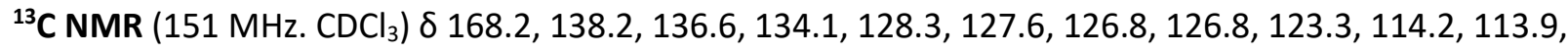
$103.4,65.3,65.3,46.0,43.7,41.2,28.4,25.9,25.3$.

HRMS: (ESI-TOF) m/z: $[\mathrm{M}+\mathrm{H}]^{+}$Calculated for $\mathrm{C}_{29} \mathrm{H}_{26} \mathrm{~N}_{3} \mathrm{O}_{4}=480.1918$, found 480.1937 . 


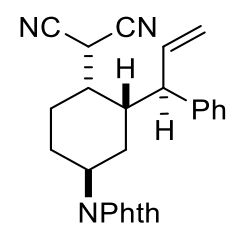

2-((1S,2R,4S)-4-(1,3-dioxoisoindolin-2-yl)-2-((R)-1-phenylallyl)cyclohexyl)malononitrile (3f)

Synthesized using General Procedure D on the $1 \mathrm{mmol}$ (407.4 mg) scale from $1 \mathrm{f}$

Note: $30 \mathrm{hr}$ reaction time, 20 equivalents $(3.86 \mathrm{~g}, 20 \mathrm{mmol}$ Hantzsch ketone. used thermometer in reaction mixture to ensure solution was at $100^{\circ} \mathrm{C}$. Recrystallized using 1:1 Ethanol/acetonitrile. Crystals grew in $-20^{\circ} \mathrm{C}$ freezer over 48 hours depending on with a concentration of approximately $10 \mathrm{~mL}$ solvent per mmol of $\mathbf{3 f}$.

Isolated: $241 \mathrm{mg}$

Yield: $60 \%$

Recrystallized yield: $65 \%, 156 \mathrm{mg}, 25: 1$ d.r.

Physical state: white solid

TLC: $\mathrm{Rf}=0.35$ in $30 \%$ ethyl acetate in hexanes, column 10-30\% ethyl acetate/hexanes

${ }^{1} \mathrm{H}$ NMR: $\left(600 \mathrm{MHz}, \mathrm{CDCl}_{3}\right) \delta 7.77(\mathrm{dd}, J=5.4,3.1 \mathrm{~Hz}, 2 \mathrm{H}), 7.71-7.64(\mathrm{~m}, 2 \mathrm{H}), 7.32(\mathrm{t}, J=7.6 \mathrm{~Hz}, 2 \mathrm{H})$, $7.29(\mathrm{dd}, J=8.2,1.6 \mathrm{~Hz}, 2 \mathrm{H}), 7.19(\mathrm{tt}, J=7.0,1.5 \mathrm{~Hz}, 1 \mathrm{H}), 6.27(\mathrm{dt}, J=17.0,10.0 \mathrm{~Hz}, 1 \mathrm{H}), 5.31(\mathrm{dd}, J=$ 17.0, $1.5 \mathrm{~Hz}, 1 \mathrm{H}), 5.13(\mathrm{dd}, J=9.9,1.4 \mathrm{~Hz}, 1 \mathrm{H}), 4.54(\mathrm{tt}, J=12.9,4.2 \mathrm{~Hz}, 1 \mathrm{H}), 4.44(\mathrm{~d}, J=12.1 \mathrm{~Hz}, 1 \mathrm{H}), 3.75$ $(\mathrm{t}, J=10.9 \mathrm{~Hz}, 1 \mathrm{H}), 2.79(\mathrm{~d}, J=11.5 \mathrm{~Hz}, 1 \mathrm{H}), 2.58(\mathrm{ddt}, J=15.6,12.5,3.1 \mathrm{~Hz}, 1 \mathrm{H}), 2.41$ (qd, $J=13.0,4.6$ $\mathrm{Hz}, 1 \mathrm{H}), 2.30(\mathrm{td}, J=13.2,4.2 \mathrm{~Hz}, 1 \mathrm{H}), 2.23-2.16(\mathrm{~m}, 1 \mathrm{H}), 2.01(\mathrm{~d}, J=13.7 \mathrm{~Hz}, 1 \mathrm{H}), 1.83(\mathrm{qd}, J=13.2,4.0$ $\mathrm{Hz}, 1 \mathrm{H}), 1.41$ (dd, $J=13.2,2.1 \mathrm{~Hz}, 1 \mathrm{H}$ ).

${ }^{13}$ C NMR: $\left(151 \mathrm{MHz}, \mathrm{CDCl}_{3}\right) \delta 173.9,168.2,142.9,141.0,138.8,133.9,132.1,129.0,128.9,128.1,127.8$, 127.6, 126.7, 123.1, 116.1, 51.5, 45.5, 40.6, 40.1, 27.2, 26.1, 2.

HRMS: (ESI-TOF) m/z: [M+Na] $]^{+}=$Calculated for $\mathrm{C}_{26} \mathrm{H}_{23} \mathrm{NaN}_{3} \mathrm{O}_{2}=432.1682$. Found 432.1696 .

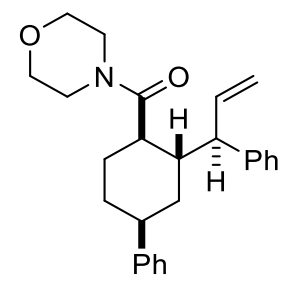

morpholino((1S,2S,4S)-4-phenyl-2-((R)-1-phenylallyl)cyclohexyl)methanone (4a)

Prepared by General Procedures D and F from 1a $(67.7 \mathrm{mg}, 0.2 \mathrm{mmol})$

Isolated: $41 \mathrm{mg}$ 
Yield: $53 \%$

Physical state: white solid

TLC: $\mathrm{Rf}=0.27$ in $20 \%$ ethyl acetate in hexanes, column $15-20 \%$ ethyl acetate in hexanes

${ }^{1}$ H NMR: $\left(600 \mathrm{MHz}, \mathrm{CDCl}_{3}\right) \delta 7.34(\mathrm{t}, J=7.7 \mathrm{~Hz}, 2 \mathrm{H}), 7.26-7.22(\mathrm{~m}, 5 \mathrm{H}), 7.18-7.11(\mathrm{~m}, 3 \mathrm{H}), 5.99(\mathrm{dt}, J=$ 17.0, $9.7 \mathrm{~Hz}, 1 \mathrm{H}), 5.17(\mathrm{dd}, J=17.2,1.7 \mathrm{~Hz}, 1 \mathrm{H}), 5.10(\mathrm{dd}, J=10.1,1.7 \mathrm{~Hz}, 1 \mathrm{H}), 3.79-3.68(\mathrm{~m}, 6 \mathrm{H}), 3.57$ $(\mathrm{t}, J=9.7 \mathrm{~Hz}, 1 \mathrm{H}), 3.51(\mathrm{~s}, 2 \mathrm{H}), 2.88(\mathrm{q}, J=4.7 \mathrm{~Hz}, 1 \mathrm{H}), 2.87-2.80(\mathrm{~m}, 1 \mathrm{H}), 2.42(\mathrm{dq}, J=9.3,4.5 \mathrm{~Hz}, 1 \mathrm{H})$, $2.20-2.14(\mathrm{~m}, 1 \mathrm{H}), 2.14-2.06(\mathrm{~m}, 1 \mathrm{H}), 1.86-1.81(\mathrm{~m}, 2 \mathrm{H}), 1.76(\mathrm{dq}, J=13.7,4.6 \mathrm{~Hz}, 1 \mathrm{H}), 1.40(\mathrm{dt}, J=$ $13.9,4.5 \mathrm{~Hz}, 1 \mathrm{H})$.

${ }^{13}$ C NMR: $\left(151 \mathrm{MHz}, \mathrm{CDCl}_{3}\right) \delta 174.8,146.3,142.7,141.7,128.9,128.1,127.1,126.7,125.9,115.7,67.2$, 66.8, 53.0, 46.4, 42.1, 39.3, 37.3, 31.4, 29..

HRMS: (ESI-TOF) Calculated for $\mathrm{m} / \mathrm{z}:[\mathrm{M}+\mathrm{H}]^{+} \mathrm{C}_{26} \mathrm{H}_{32} \mathrm{NO}_{2}=390.2433$. Found 390.2445.<smiles>C=CC(c1ccccc1)[C@H]1C[C@@H](c2ccccc2)CCC1C(=O)N(C)OC</smiles>

$(1 \mathrm{~S}, 2 \mathrm{~S}, 4 \mathrm{~S})-\mathrm{N}$-methoxy-N-methyl-4-phenyl-2-((R)-1-phenylallyl)cyclohexane-1-carboxamide 4b

Prepared using General Procedures D and F from 1a $(67.7 \mathrm{mg}, 0.2 \mathrm{mmol})$

Isolated: $28 \mathrm{mg}$

Yield: $39 \%$

Appearance: White solid

TLC: $\mathrm{Rf}=0.27$ in $20 \%$ Ethyl acetate/hexanes

${ }^{1} \mathrm{H}$ NMR $\left(600 \mathrm{MHz}, \mathrm{CDCl}_{3}\right) \delta 7.32-7.26(\mathrm{~m}, 3 \mathrm{H}), 7.21-7.18(\mathrm{~m}, 1 \mathrm{H}), 7.17-7.10(\mathrm{~m}, 2 \mathrm{H}), 5.99$ (ddd, $J=$ 16.9, 10.1, 8.6 Hz, 1H), $5.04(\mathrm{dt}, J=16.9,1.4 \mathrm{~Hz}, 1 \mathrm{H}), 4.97(\mathrm{dd}, J=10.0,1.8 \mathrm{~Hz}, 1 \mathrm{H}), 3.67$ (ddt, $J=57.2$, 22.1, $5.8 \mathrm{~Hz}, 4 \mathrm{H}), 3.49(\mathrm{dd}, J=16.7,8.1 \mathrm{~Hz}, 1 \mathrm{H}), 3.42-3.31(\mathrm{~m}, 2 \mathrm{H}), 3.29(\mathrm{t}, J=8.4 \mathrm{~Hz}, 1 \mathrm{H}), 2.43(\mathrm{ddt}, J=$ $11.8,8.3,3.8 \mathrm{~Hz}, 1 \mathrm{H}$ ), 2.38 (td, $J=8.2,4.7 \mathrm{~Hz}, 1 \mathrm{H}$ ), 1.72 (dddd, $J=22.2,10.7,5.2,2.7 \mathrm{~Hz}, 2 \mathrm{H}$ ), 1.63 (dddd, $J=12.4,10.5,8.3,4.6 \mathrm{~Hz}, 2 \mathrm{H}), 1.52(\mathrm{ddt}, J=13.8,6.5,4.4 \mathrm{~Hz}, 1 \mathrm{H}), 1.33(\mathrm{ddt}, J=14.0,10.1,4.1 \mathrm{~Hz}, 1 \mathrm{H})$, $1.20(\mathrm{ddt}, J=9.8,7.6,4.0 \mathrm{~Hz}, 1 \mathrm{H}$ ), 1.06 (ddd, $J=13.4,9.4,3.8 \mathrm{~Hz}, 1 \mathrm{H}), 0.78(\mathrm{~d}, J=6.6 \mathrm{~Hz}, 3 \mathrm{H}), 0.72(\mathrm{~d}, J=$ $6.6 \mathrm{~Hz}, 3 \mathrm{H})$.

${ }^{13} \mathrm{C}$ NMR $\left(151 \mathrm{MHz} \mathrm{CDCl}_{3}\right) \delta 175.1,142.6,142.0,128.5,126.5,114.8,67.1,66.7,54.4,46.0,42.0,41.1$, $39.3,38.5,29.2,28.1,27.1,25.0,20.8,20.5$.

HRMS: (ESI-TOF) m/z: [M+Na] $]^{+}$Calculated for $\mathrm{C}_{24} \mathrm{H}_{29} \mathrm{NaNO}_{2}=386.2091$. Found 386.2086. 


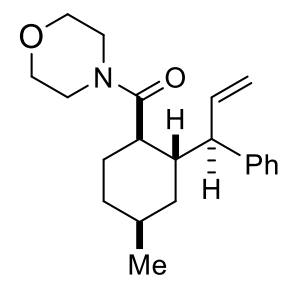

((1S,2S,4S)-4-methyl-2-((R)-1-phenylallyl)cyclohexyl)(morpholino)methanone (4c)

Prepared using General Procedures D and F from 1a $(52.4 \mathrm{mg}, 0.2 \mathrm{mmol})$

Isolated: $30 \mathrm{mg}$

Yield: $46 \%$

Physical state: white solid

TLC: $R_{f}=0.48$ in $50 \%$ ethyl acetate/hexanes

${ }^{1} \mathbf{H}$ NMR $\left(600 \mathrm{MHz}, \mathrm{CDCl}_{3}\right) \delta 7.31-7.24(\mathrm{~m}, 2 \mathrm{H}), 7.14-7.09(\mathrm{~m}, 2 \mathrm{H}), 5.99$ (ddd, J = 17.0, 10.1, 8.2 Hz, $1 \mathrm{H}), 5.03(\mathrm{dt}, J=17.1,1.5 \mathrm{~Hz}, 1 \mathrm{H}), 4.99-4.95(\mathrm{~m}, 1 \mathrm{H}), 3.78-3.72(\mathrm{~m}, 2 \mathrm{H}), 3.72-3.62(\mathrm{~m}, 1 \mathrm{H}), 3.61-$ $3.55(\mathrm{~m}, 1 \mathrm{H}), 3.55-3.43(\mathrm{~m}, 1 \mathrm{H}), 3.34(\mathrm{t}, J=8.0 \mathrm{~Hz}, 2 \mathrm{H}), 2.51$ (ddq, $J=14.2,8.0,3.8 \mathrm{~Hz}, 1 \mathrm{H}$ ), 2.27 (ddd, $J$ $=9.7,8.2,4.3 \mathrm{~Hz}, 1 \mathrm{H}), 1.91-1.82(\mathrm{~m}, 1 \mathrm{H}), 1.69(\mathrm{dtd}, J=13.7,10.2,4.0 \mathrm{~Hz}, 1 \mathrm{H}), 1.56(\mathrm{dt}, J=13.4,4.8 \mathrm{~Hz}$, $1 \mathrm{H}), 1.50(\mathrm{dq}, J=13.5,4.5 \mathrm{~Hz}, 1 \mathrm{H}), 1.45(\mathrm{dq}, J=9.7,4.9 \mathrm{~Hz}, 1 \mathrm{H}), 1.40(\mathrm{ddt}, J=13.2,10.9,4.4 \mathrm{~Hz}, 1 \mathrm{H}), 1.16$ (ddd, $J=13.9,10.2,4.2 \mathrm{~Hz}, 1 \mathrm{H}), 0.92(\mathrm{~d}, J=7.0 \mathrm{~Hz}, 3 \mathrm{H}$ ).

${ }^{13} \mathrm{C}$ NMR $\left(151 \mathrm{MHz}, \mathrm{CDCl}_{3}\right) \delta 174.9,142.5,142.0,128.8,128.4,126.4,114.7,67.4,66.7,53.9,45.9,41.98$, 41.7, 37.7, 33.1, 30.9, 26.9, 24.7, 18.8 .

HRMS: (ESI-TOF) m/z: m/z: [M+H] ${ }^{+}$Calculated for $\mathrm{C}_{21} \mathrm{H}_{30} \mathrm{NO}_{2}: 328.2271$. Found 328.2278.<smiles>[M]C1CCC(C(=O)N(C)OC)C(C(C=C)c2ccccc2)C1</smiles>

(1S,2S,4S)-N-methoxy-N,4-dimethyl-2-((R)-1-phenylallyl)cyclohexane-1-carboxamide (4d) Prepared using Procedures D and F from 1b $(55 \mathrm{mg}, 0.2 \mathrm{mmol})$

Isolated: $11.5 \mathrm{mg}$

Yield: $19 \%$

Physical state: white solid

TLC: $R_{f}=0.57$ in $30 \%$ Ethyl acetate in hexanes purified with $0-15 \%$ ethyl acetate/hexanes

${ }^{1} \mathbf{H}$ NMR $\left(600 \mathrm{MHz}, \mathrm{CDCl}_{3}\right) \delta 7.31-7.25(\mathrm{~m}, 3 \mathrm{H}), 7.25-7.12(\mathrm{~m}, 3 \mathrm{H}), 5.96$ (ddd, $J=17.0,10.1,9.0 \mathrm{~Hz}$, $1 \mathrm{H}), 5.08-4.99(\mathrm{~m}, 1 \mathrm{H}), 4.96(\mathrm{dd}, J=10.0,1.8 \mathrm{~Hz}, 1 \mathrm{H}), 3.64(\mathrm{~s}, 3 \mathrm{H}), 3.29(\mathrm{t}, J=9.1 \mathrm{~Hz}, 1 \mathrm{H}), 3.16(\mathrm{~s}, 3 \mathrm{H})$, 
$2.41(\mathrm{tdd}, J=8.7,7.0,4.0 \mathrm{~Hz}, 1 \mathrm{H}), 1.82-1.69(\mathrm{~m}, 2 \mathrm{H}), 1.69-1.60(\mathrm{~m}, 1 \mathrm{H}), 1.54-1.44(\mathrm{~m}, 2 \mathrm{H}), 1.44-$ $1.36(\mathrm{~m}, 1 \mathrm{H}), 1.12$ (ddd, $J=13.2,8.5,4.1 \mathrm{~Hz}, 1 \mathrm{H}), 0.86(\mathrm{~d}, J=6.9 \mathrm{~Hz}, 3 \mathrm{H})$.

${ }^{13} \mathrm{C}$ NMR $\left(151 \mathrm{MHz}, \mathrm{CDCl}_{3}\right)$ $\delta$ 143.2, 142.1, 128.4, 126.2, 114.34 61.1, 54.5, 40.7, 37.8, 33.6, 31.0, 29.7, 26.7, 24.3, 19.6.

HRMS: (ESI-TOF) m/z: [M+Na] $]^{+}$Calculated for $\mathrm{C}_{19} \mathrm{H}_{27} \mathrm{NO}_{2} \mathrm{Na}=324.1934$, found 324.1936.

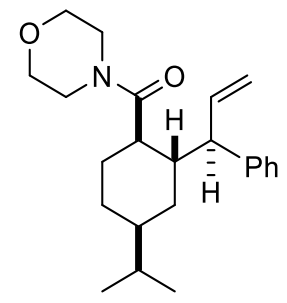

((1S,2S,4S)-4-isopropyl-2-((R)-1-phenylallyl)cyclohexyl)(morpholino)methanone (4e)

Prepared using Procedures D and F from 1c (58.0 mg, $0.2 \mathrm{mmol})$

Isolated" $36 \mathrm{mg}$

Yield: $62 \%$ yield

Appearance: White solid

TLC: $R_{f}=0.22$ in $20 \%$ ethyl acetate hexanes

${ }^{1} \mathbf{H}$ NMR $\left(600 \mathrm{MHz}, \mathrm{CDCl}_{3}\right) \delta 7.32-7.26(\mathrm{~m}, 3 \mathrm{H}), 7.21-7.18(\mathrm{~m}, 1 \mathrm{H}), 7.17-7.10(\mathrm{~m}, 2 \mathrm{H}), 5.99$ (ddd, $J=$ 16.9, 10.1, 8.6 Hz, 1H), 5.04 (dt, $J=16.9,1.4 \mathrm{~Hz}, 1 \mathrm{H}), 4.97$ (dd, $J=10.0,1.8 \mathrm{~Hz}, 1 \mathrm{H}$ ), 3.67 (ddt, $J=57.2$, 22.1, $5.8 \mathrm{~Hz}, 4 \mathrm{H}), 3.49(\mathrm{dd}, J=16.7,8.1 \mathrm{~Hz}, 1 \mathrm{H}), 3.42-3.31(\mathrm{~m}, 2 \mathrm{H}), 3.29(\mathrm{t}, J=8.4 \mathrm{~Hz}, 1 \mathrm{H}), 2.43(\mathrm{ddt}, J=$ $11.8,8.3,3.8 \mathrm{~Hz}, 1 \mathrm{H}$ ), 2.38 (td, $J=8.2,4.7 \mathrm{~Hz}, 1 \mathrm{H}$ ), 1.72 (dddd, $J=22.2,10.7,5.2,2.7 \mathrm{~Hz}, 2 \mathrm{H}$ ), 1.63 (dddd, $J=12.4,10.5,8.3,4.6 \mathrm{~Hz}, 2 \mathrm{H}), 1.52(\mathrm{ddt}, J=13.8,6.5,4.4 \mathrm{~Hz}, 1 \mathrm{H}), 1.33(\mathrm{ddt}, J=14.0,10.1,4.1 \mathrm{~Hz}, 1 \mathrm{H}$ ), $1.20(\mathrm{ddt}, J=9.8,7.6,4.0 \mathrm{~Hz}, 1 \mathrm{H}), 1.06(\mathrm{ddd}, J=13.4,9.4,3.8 \mathrm{~Hz}, 1 \mathrm{H}), 0.78(\mathrm{~d}, J=6.6 \mathrm{~Hz}, 3 \mathrm{H}), 0.72(\mathrm{~d}, J=$ $6.6 \mathrm{~Hz}, 3 \mathrm{H})$.

${ }^{13} \mathrm{C}$ NMR $\left(151 \mathrm{MHz}, \mathrm{CDCl}_{3}\right) \delta 175.1,142.6,142.0,128.5,126.5,114.8,67.1,66.7,54.4,46.0,42.0,41.1$, $39.3,38.5,29.2,28.0,27.1,25.0,20.8,20.5$.

HRMS: (ESI-TOF) m/z: $[\mathrm{M}+\mathrm{H}]^{+}$Calculated for $\mathrm{C}_{23} \mathrm{H}_{33} \mathrm{NO}_{2}=356.2584$. Found 356.2584.

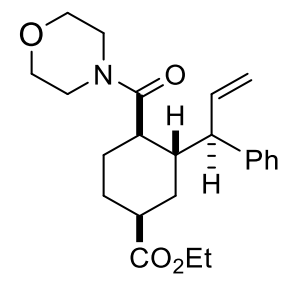

ethyl (1S,3S,4S)-4-(morpholine-4-carbonyl)-3-((R)-1-phenylallyl)cyclohexane-1-carboxylate (4f)

Prepared using General Procedure D and F, from of 1d (66.9 mg $0.2 \mathrm{mmol})$

Note: Hydrolysis was not performed ester would also be hydrolyzed. 
Isolated: $45.3 \mathrm{mg}$

Yield: 59\%

Physical state: off-white solid

TLC: $\mathbf{R}_{\mathbf{f}}=0.29$ in $30 \%$ ethyl acetate/hexanes. Column chromatography: 0-30\% ethyl acetate/hexanes

${ }^{1}$ H NMR: $\left(600 \mathrm{MHz}, \mathrm{CDCl}_{3}\right) \delta 7.28(\mathrm{t}, J=7.6 \mathrm{~Hz}, 2 \mathrm{H}), 7.23-7.18(\mathrm{~m}, 1 \mathrm{H}), 7.13-7.09(\mathrm{~m}, 2 \mathrm{H}), 6.01$ (ddd, $J$ $=17.4,10.3,7.4 \mathrm{~Hz}, 1 \mathrm{H}), 5.07(\mathrm{dt}, J=17.1,1.6 \mathrm{~Hz}, 1 \mathrm{H}), 5.01(\mathrm{dt}, J=10.3,1.5 \mathrm{~Hz}, 1 \mathrm{H}), 4.17(\mathrm{dq}, J=10.7$, $7.1 \mathrm{~Hz}, 1 \mathrm{H}), 4.09(\mathrm{dq}, J=10.7,7.1 \mathrm{~Hz}, 1 \mathrm{H}), 3.79-3.70(\mathrm{~m}, 2 \mathrm{H}), 3.71-3.60(\mathrm{~m}, 1 \mathrm{H}), 3.56(\mathrm{ddd}, J=11.1$, 7.4, $3.5 \mathrm{~Hz}, 1 \mathrm{H}$ ), 3.50 (ddd, $J=12.9,10.0,4.1 \mathrm{~Hz}, 1 \mathrm{H}), 3.37(\mathrm{t}, J=7.0 \mathrm{~Hz}, 1 \mathrm{H}), 3.36-3.24(\mathrm{~m}, 2 \mathrm{H}), 2.62(\mathrm{p}$, $J=4.2 \mathrm{~Hz}, 1 \mathrm{H}$ ), 2.50 (dddd, $J=12.1,9.7,6.4,3.4 \mathrm{~Hz}, 1 \mathrm{H}$ ), 2.28 (dtd, $J=13.7,3.7,1.7 \mathrm{~Hz}, 1 \mathrm{H}$ ), 2.15 (ddd, $J$ $=13.6,6.8,4.2 \mathrm{~Hz}, 2 \mathrm{H}), 1.68-1.59(\mathrm{~m}, 1 \mathrm{H}), 1.57(\mathrm{tt}, J=13.5,4.0 \mathrm{~Hz}, 1 \mathrm{H}), 1.34(\mathrm{ddt}, J=12.9,8.4,4.6 \mathrm{~Hz}$, $1 \mathrm{H}), 1.24(\mathrm{t}, J=7.1 \mathrm{~Hz}, 4 \mathrm{H}), 1.22-1.09(\mathrm{~m}, 1 \mathrm{H})$.

${ }^{13}$ C NMR: $\left(151 \mathrm{MHz}, \mathrm{CDCl}_{3}\right) \delta$ 174.1, 141.7, 141.2, 129.1, 128.3, 126.6, 114.8, 67.0, 66.6, 60.5, 53.2, 45.8, 42.2, $39.3338 .9,29.8,28.3,26.8,26.3,14.3$.

HRMS: (ESI-TOF) m/z: m/z: [M+Na] $]^{+} . \mathrm{C}_{23} \mathrm{H}_{31} \mathrm{NNaO}_{4}: 408.2145$, found 408.2153.<smiles>C=CC(c1ccccc1)[C@H]1CC(C(C)=O)CCC1C(=O)N(C)OC</smiles>

Ethyl (1S,3S,4S)-4-(methoxy(methyl)carbamoyl)-3-((R)-1-phenylallyl)cyclohexane-1-carboxylate (4g) Prepared using Procedures $\mathbf{D}$ and $\mathbf{F}$, from $66.9 \mathrm{mg}(0.2 \mathrm{mmol})$ of $\mathbf{1 d}$

Note: Hydrolysis was not performed, ester would also be hydrolyzed.

Isolated: $18.2 \mathrm{mg}$

Yield: $25 \%$ yield

Physical state: white solid

TLC: $R_{f}=0.25$ in $50 \%$ ethyl acetate/hexanes

${ }^{1}$ H NMR $\left(600 \mathrm{MHz}, \mathrm{CDCl}_{3}\right) \delta 7.31-7.24(\mathrm{~m}, 3 \mathrm{H}), 7.17-7.14(\mathrm{~m}, 2 \mathrm{H}), 5.97$ (ddd, J = 17.1, 10.1, 8.4 Hz, $1 \mathrm{H}), 5.05(\mathrm{dt}, J=17.1,1.4 \mathrm{~Hz}, 1 \mathrm{H}), 4.98(\mathrm{dt}, J=10.1,1.2 \mathrm{~Hz}, 1 \mathrm{H}), 4.11(\mathrm{ddq}, J=37.5,10.8,7.1 \mathrm{~Hz}, 2 \mathrm{H}$ ), $3.62(\mathrm{~s}, 3 \mathrm{H}), 3.27(\mathrm{t}, J=8.1 \mathrm{~Hz}, 1 \mathrm{H}), 3.14(\mathrm{~s}, 3 \mathrm{H}), 2.59(\mathrm{p}, J=4.7 \mathrm{~Hz}, 1 \mathrm{H}), 2.44(\mathrm{dtd}, J=11.0,8.3,3.6 \mathrm{~Hz}$, $1 \mathrm{H}$ ), 2.17 (ddd, $J=13.4,6.8,2.9 \mathrm{~Hz}, 1 \mathrm{H}$ ), $2.15-2.10(\mathrm{~m}, 1 \mathrm{H}), 1.73(\mathrm{dq}, J=13.6,4.4 \mathrm{~Hz}, 1 \mathrm{H}$ ), 1.64 (dddd, $J$ $=13.8,11.5,10.0,3.8 \mathrm{~Hz}, 1 \mathrm{H}), 1.51-1.43(\mathrm{~m}, 1 \mathrm{H}), 1.23(\mathrm{t}, J=7.1 \mathrm{~Hz}, 3 \mathrm{H}), 1.17$ (ddd, $J=13.8,10.6,4.6$ $\mathrm{Hz}, 1 \mathrm{H})$.

${ }^{13} \mathrm{C}$ NMR $\left(151 \mathrm{MHz}, \mathrm{CDCl}_{3}\right) \delta 174.6,141.7,128.7,128.5,126.5,114.6,61.3,60.5,54.6,39.0,38.9,29.02$, 26.6, 26.4, 14.4.

HRMS: (ESI-TOF) m/z: m/z: [M+H] $]^{+}$. Calculated for $\mathrm{C}_{21} \mathrm{H}_{30} \mathrm{NO}_{4}: 360.2169$ found: 360.2182 . 


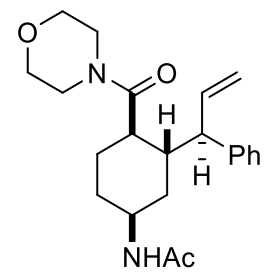

$\mathrm{N}-((1 \mathrm{~S}, 3 \mathrm{~S}, 4 \mathrm{~S})-4-($ morpholine-4-carbonyl)-3-((R)-1-phenylallyl)cyclohexyl)acetamide (4h)

Prepared using Procedures D and F from 1e (61.0 mg, $0.2 \mathrm{mmol})$

Isolated: $30.1 \mathrm{mg}$

Yield: $41 \%$

Physical state: white solid

TLC: $R_{f}=0.14$ in $5 \% \mathrm{MeOH} / \mathrm{DCM}$; Column 2.5 to $5 \%$ ethyl acetate/hexanes

${ }^{1} \mathrm{H}$ NMR $\left(600 \mathrm{MHz}, \mathrm{CDCl}_{3}\right) \delta 7.28(\mathrm{t}, J=7.6 \mathrm{~Hz}, 2 \mathrm{H}), 7.23-7.18(\mathrm{~m}, 1 \mathrm{H}), 7.13-7.08(\mathrm{~m}, 2 \mathrm{H}), 6.01$ (ddd, $J=$ 17.4, 10.3, 7.4 Hz, 1H), 5.07 (dt, $J=17.1,1.6 \mathrm{~Hz}, 1 \mathrm{H}$ ), 5.01 (dt, $J=10.3,1.5 \mathrm{~Hz}, 1 \mathrm{H}$ ), 4.17 (dq, $J=10.7,7.1$ $\mathrm{Hz}, 1 \mathrm{H}), 4.09(\mathrm{dq}, J=10.7,7.1 \mathrm{~Hz}, 1 \mathrm{H}), 3.79-3.70(\mathrm{~m}, 2 \mathrm{H}), 3.71-3.60(\mathrm{~m}, 1 \mathrm{H}), 3.56$ (ddd, $J=11.1,7.4$, $3.5 \mathrm{~Hz}, 1 \mathrm{H}), 3.50$ (ddd, $J=12.9,10.0,4.1 \mathrm{~Hz}, 1 \mathrm{H}), 3.37(\mathrm{t}, J=7.0 \mathrm{~Hz}, 1 \mathrm{H}), 3.36-3.24(\mathrm{~m}, 2 \mathrm{H}), 2.62(\mathrm{p}, J=$ $4.2 \mathrm{~Hz}, 1 \mathrm{H}$ ), 2.50 (dddd, $J=12.1,9.7,6.4,3.4 \mathrm{~Hz}, 1 \mathrm{H}$ ), 2.28 (dtd, $J=13.7,3.7,1.7 \mathrm{~Hz}, 1 \mathrm{H}$ ), 2.15 (ddd, $J=$ 13.6, 6.8, 4.2 Hz, 2H), $1.68-1.59(\mathrm{~m}, 1 \mathrm{H}), 1.57(\mathrm{tt}, J=13.5,4.0 \mathrm{~Hz}, 1 \mathrm{H}), 1.34$ (ddt, $J=12.9,8.4,4.6 \mathrm{~Hz}$, $1 \mathrm{H}), 1.24(\mathrm{t}, J=7.1 \mathrm{~Hz}, 4 \mathrm{H}), 1.22-1.09(\mathrm{~m}, 1 \mathrm{H})$.

${ }^{13} \mathrm{C}$ NMR $\left(151 \mathrm{MHz}, \mathrm{CDCl}_{3}\right) \delta 174.1,141.7,141.2,129.1,128.4,126.6,114.8,67.0,66.6,60.5,53.2,45.78$, $42.2,39.3,38.9,29.8,28.3,26.8,26.3,14.3$.

HRMS: (ESI-TOF) m/z: $[\mathrm{M}+\mathrm{H}]^{+}=$Calculated for $\mathrm{C}_{22} \mathrm{H}_{31} \mathrm{~N}_{2} \mathrm{O}_{3}=371.2329$ Found 371.2340<smiles>C=CC(c1ccccc1)C1CC(N)CCC1C(=O)N(C)OC</smiles>

(1S,2S,4S)-4-acetamido-N-methoxy-N-methyl-2-((R)-1-phenylallyl)cyclohexane-1-carboxamide (4i)

Prepared using Procedures D and F from 1e $(61.0 \mathrm{mg}, 0.2 \mathrm{mmol})$

Isolated: $11.7 \mathrm{mg}$

Yield: $17 \%$

Appearance: White solid

TLC: $R_{f}=0.19$ in $5 \% \mathrm{MeOH} / \mathrm{DCM}$.

${ }^{1} \mathrm{H}$ NMR $\left(400 \mathrm{MHz}, \mathrm{CDCl}_{3}\right) \delta 7.33-7.26(\mathrm{~m}, 2 \mathrm{H}), 7.21-7.17(\mathrm{~m}, 1 \mathrm{H}), 7.17-7.12(\mathrm{~m}, 2 \mathrm{H}), 5.97$ (ddd, $J=$ 17.0, 10.1, 8.8 Hz, 1H), 5.68 (d, $J=8.1 \mathrm{~Hz}, 1 \mathrm{H}$ ), 5.04 (ddd, $J=17.0,1.7,0.9 \mathrm{~Hz}, 1 \mathrm{H}$ ), 4.99 (ddd, $1 \mathrm{H}$ ), 4.14 
$(\mathrm{q}, J=4.2 \mathrm{~Hz}, 1 \mathrm{H}), 3.63(\mathrm{~s}, 3 \mathrm{H}), 3.30(\mathrm{t}, J=8.4 \mathrm{~Hz}, 1 \mathrm{H}), 3.16(\mathrm{~s}, 3 \mathrm{H}), 2.69(\mathrm{~s}, 1 \mathrm{H}), 2.47-2.31(\mathrm{~m}, 1 \mathrm{H}), 1.94$ $(\mathrm{s}, 3 \mathrm{H}), 1.81-1.67(\mathrm{~m}, 1 \mathrm{H}), 1.65(\mathrm{~d}, J=7.8 \mathrm{~Hz}, \mathrm{OH})$.

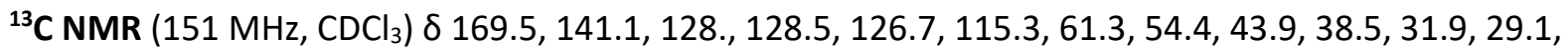
24.6, 23.8 .

HRMS: (ESI-TOF) m/z: [M+Na] Calculated for $\mathrm{m} / \mathrm{z}$ : $[\mathrm{M}+\mathrm{Na}]^{+} \mathrm{C}_{20} \mathrm{H}_{28} \mathrm{~N}_{2} \mathrm{NaO}_{3}=367.1992$, Found 367.1992.

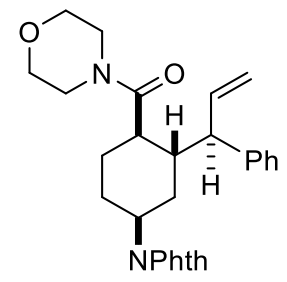

2-((1S,3S,4S)-4-(morpholine-4-carbonyl)-3-((R)-1-phenylallyl)cyclohexyl)isoindoline-1,3-dione (4j)

Prepared using Procedures E and G from $1 f(81.5 \mathrm{mg}, 0.2 \mathrm{mmol})$

Isolated: $61.3 \mathrm{mg}$

Yield: $67 \%$

Physical state: white solid

TLC: $\mathbf{R}_{\mathbf{f}}=0.11$ in $30 \%$ ethyl acetate/hexanes. Column: $30 \%$ to $50 \%$ ethyl acetate/hexanes

${ }^{1} \mathrm{H}$ NMR $\left(600 \mathrm{MHz}, \mathrm{CDCl}_{3}\right) \delta 7.78-7.71(\mathrm{~m}, 2 \mathrm{H}), 7.64(\mathrm{dd}, J=5.5,3.0 \mathrm{~Hz}, 2 \mathrm{H}), 7.29(\mathrm{dd}, J=8.1,7.1 \mathrm{~Hz}$, $2 \mathrm{H}), 7.28-7.23(\mathrm{~m}, 1 \mathrm{H}), 7.16(\mathrm{tt}, J=7.2,1.4 \mathrm{~Hz}, 1 \mathrm{H}), 5.94(\mathrm{dt}, J=17.0,9.9 \mathrm{~Hz}, 1 \mathrm{H}), 5.18(\mathrm{dd}, J=17.0,1.6$ $\mathrm{Hz}, 1 \mathrm{H}), 5.09(\mathrm{dd}, J=10.0,1.6 \mathrm{~Hz}, 1 \mathrm{H}), 4.40(\mathrm{tdt}, J=12.4,8.3,4.2 \mathrm{~Hz}, 1 \mathrm{H}), 3.71(\mathrm{~s}, 5 \mathrm{H}), 3.62(\mathrm{~s}, 0 \mathrm{H}), 3.56$ (dd, $J=11.7,9.7 \mathrm{~Hz}, 1 \mathrm{H}), 3.51(\mathrm{~s}, 2 \mathrm{H}), 2.99(\mathrm{p}, J=2.7 \mathrm{~Hz}, 1 \mathrm{H}), 2.78(\mathrm{td}, J=13.1,5.2 \mathrm{~Hz}, 1 \mathrm{H}), 2.70(\mathrm{td}, J=$ $12.3,4.8 \mathrm{~Hz}, 1 \mathrm{H}), 2.46(\mathrm{ddt}, J=11.3,5.1,2.5 \mathrm{~Hz}, 1 \mathrm{H}), 1.89(\mathrm{dtt}, J=26.2,14.0,4.2 \mathrm{~Hz}, 2 \mathrm{H}), 1.72-1.58(\mathrm{~m}$, $3 \mathrm{H}), 1.18(\mathrm{dd}, J=13.1,4.1 \mathrm{~Hz}, 1 \mathrm{H})$.

${ }^{13} \mathrm{C}$ NMR $\left(151 \mathrm{MHz}, \mathrm{CDCl}_{3}\right) \delta 174.1,168.1,142.6,141.0,133.7,132.1,129.0,127.8,126.7,123.0,116.3$, $67.2,66.8,52.2,46.6,45.6,42.3,40.2,35.4,26.9,25.8,23.6$.

HRMS: (ESI-TOF) m/z: m/z: [M+H] ${ }^{+}$Calculated for $\mathrm{C}_{22} \mathrm{H}_{31} \mathrm{~N}_{2} \mathrm{O}_{3}: 371.2329$. Found 371.2340.

$3 \mathrm{mmol}$ scale in $\mathbf{3} \times 1 \mathrm{mmol}$ reactions: Three $50 \mathrm{~mL}$ Schlenk flasks were prepared as follows: The Schlenk flasks were flame-dried and charged with Hantzsch ketone ( $3.86 \mathrm{~g}, 20 \mathrm{mmol}, 20$ equiv.) and diene $\mathbf{1 f}$ ( $407.5 \mathrm{mg}, 1 \mathrm{mmol}, 1$ equiv.). The flasks were evacuated and backfilled with nitrogen three times, then dry DMF (10 mL, $0.1 \mathrm{M})$ was added. The reactions were heated in a preheated oil bath at $100^{\circ} \mathrm{C}$ with temperatures being measured with a thermometer in the reaction flasks. The reactions were stirred for $20 \mathrm{hr}$, with an aliquot NMR analysis indicating completion. Upon completion, the flasks were cooled in an ice bath to precipitate the Hantzsch ketone. The reactions were vacuum filtered to separate reductant and the solution, washing with ethyl acetate. The filtrate was added to a separatory funnel and washed with $2 \mathrm{~N}$ aq. $\mathrm{HCl}(150 \mathrm{~mL})$. The aqueous layer was extracted with ethyl acetate $(3 \times 40 \mathrm{~mL})$. The combined organics were washed with brine, dried with $\mathrm{Na}_{2} \mathrm{SO}_{4}$ and concentrated. Hantzsch ketone was recrystallized from hot ethanol (approx. $200 \mathrm{~mL}$ ) and $8.3 \mathrm{~g}(43.1 \mathrm{mmol}, 75 \%$ recovery). In a $200 \mathrm{~mL}$ Schlenk flask, Crude $\mathbf{3 f}$ (ran as $1.23 \mathrm{~g}, 3 \mathrm{mmol}$ ) was subsequently dissolved in 3:1 MeCN/DMSO (0.05 M). 
Morpholine $(1.31 \mathrm{~g}, 1.29 \mathrm{~mL}, 15 \mathrm{mmol})$ and $\mathrm{K}_{2} \mathrm{CO}_{3}(2.07 \mathrm{~g}, 15 \mathrm{mmol})$ were then added. Three balloons of oxygen were bubbled into the solution and the reaction was allowed to stir overnight. Upon completion per an aliquot NMR, the reaction mixture was vacuum filtered through a silica plug, flushing with ethyl acetate (approx.. $100 \mathrm{~mL}$ ). DMSO was removed by washing the organic layer with $2 \mathrm{~N}$ aq. $\mathrm{HCl}(3 \times 30 \mathrm{~mL}$ ). The organic layer was washed with brine, dried with $\mathrm{Na}_{2} \mathrm{SO}_{4}$, and concentrated. Amide $4 \mathbf{j}$ was isolated via column chromatography (20-30-50-80-100\% ethyl acetate/hexanes). Isolated $0.9694 \mathrm{~g}, 71 \%$ yield.<smiles>C=CC(c1ccccc1)[C@H]1CC([NH+])CCC1C(=O)N(C)OC</smiles>

(1S,2S,4S)-4-(1,3-dioxoisoindolin-2-yl)-N-methoxy-N-methyl-2-((R)-1-phenylallyl)cyclohexane-1carboxamide (4k)

Prepared by using Procedure E and G from $1 \mathrm{f}(81.5 \mathrm{mg}, 0.2 \mathrm{mmol})$

Isolated: $57.9 \mathrm{mg}$

Yield: $64 \%$

Physical state: white solid

TLC: $\mathrm{Rf}=0.48$ in $50 \%$ ethyl acetate/hexanes, Column: column $30-40 \%$ ethyl acetate/hexanes

${ }^{1} \mathrm{H}$ NMR $\left(600 \mathrm{MHz}, \mathrm{CDCl}_{3}\right) \delta 7.74(\mathrm{dd}, J=5.4,3.0 \mathrm{~Hz}, 2 \mathrm{H}), 7.65-7.61(\mathrm{~m}, 2 \mathrm{H}), 7.28(\mathrm{~d}, J=5.3 \mathrm{~Hz}, 3 \mathrm{H}), 7.17$ $-7.13(\mathrm{~m}, 1 \mathrm{H}), 5.98(\mathrm{dt}, J=17.0,9.8 \mathrm{~Hz}, 1 \mathrm{H}), 5.17(\mathrm{dd}, J=16.9,1.7 \mathrm{~Hz}, 1 \mathrm{H}), 5.09(\mathrm{dd}, J=10.0,1.6 \mathrm{~Hz}, 1 \mathrm{H})$, $4.40(\mathrm{tt}, J=12.6,4.0 \mathrm{~Hz}, 1 \mathrm{H}), 3.71(\mathrm{~s}, 3 \mathrm{H}), 3.59(\mathrm{dd}, J=11.8,9.7 \mathrm{~Hz}, 1 \mathrm{H}), 3.24(\mathrm{~s}, 3 \mathrm{H}), 3.15-3.12(\mathrm{~m}, 1 \mathrm{H})$, $2.82(\mathrm{qd}, J=12.7,4.4 \mathrm{~Hz}, 1 \mathrm{H}), 2.66(\mathrm{td}, J=13.2,5.1 \mathrm{~Hz}, 1 \mathrm{H}), 2.51(\mathrm{~d}, J=11.3 \mathrm{~Hz}, 1 \mathrm{H}), 2.01$ (dd, $J=14.4$, $3.3 \mathrm{~Hz}, 1 \mathrm{H}), 1.85(\mathrm{tt}, J=13.9,5.0 \mathrm{~Hz}, 1 \mathrm{H}), 1.70-1.61(\mathrm{~m}, 0 \mathrm{H}), 1.15(\mathrm{~d}, J=13.2 \mathrm{~Hz}, 1 \mathrm{H})$.

${ }^{13}$ C NMR: $\left(151 \mathrm{MHz}, \mathrm{CDCl}_{3}\right) \delta 168.2,143.1,141.5,133.7,132.2,129.0,127.8,126.6,123.1,115.7,61.3$, 52.0, 45.7, 40.2, 35.6, 26.9, 26.0, 22.8.

HRMS: (ESI-TOF) m/z: $[\mathrm{M}+\mathrm{H}]^{+}$Calculated for $\mathrm{C}_{26} \mathrm{H}_{29} \mathrm{~N}_{2} \mathrm{O}_{4}=433.2122$. Found 433.2120.

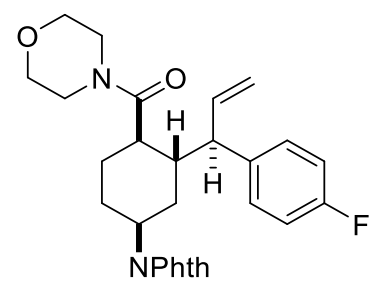

2-((1S,3S,4R)-3-((R)-1-(4-fluorophenyl)allyl)-4-(morpholine-4-carbonyl)cyclohexyl)isoindoline-1,3-dione (4I)

Prepared using Procedures E and G from $1 \mathrm{~h}(85.1 \mathrm{mg}, 0.2 \mathrm{mmol})$

Isolated: $47.5 \mathrm{mg}$ 
Yield: $50 \%$

Physical state: white solid

TLC: $R_{f}=0.27$ in $50 \%$ ethyl acetate/hexanes. Column in $50 \%$ ethyl acetate/hexanes

${ }^{1} \mathbf{H}$ NMR $\left(600 \mathrm{MHz}, \mathrm{CDCl}_{3}\right) \delta 7.8-7.7(\mathrm{~m}, 2 \mathrm{H}), 7.7-7.6(\mathrm{~m}, 2 \mathrm{H}), 7.2-7.2(\mathrm{~m}, 2 \mathrm{H}), 7.0-6.9(\mathrm{~m}, 2 \mathrm{H}), 5.9$ $(\mathrm{dt}, J=17.0,9.8 \mathrm{~Hz}, 1 \mathrm{H}), 5.2(\mathrm{dd}, J=16.9,1.6 \mathrm{~Hz}, 1 \mathrm{H}), 5.1-5.1(\mathrm{~m}, 1 \mathrm{H}), 4.3(\mathrm{tt}, J=12.4,4.2 \mathrm{~Hz}, 1 \mathrm{H}), 3.8-$ $3.6(\mathrm{~m}, 5 \mathrm{H}), 3.5(\mathrm{dd}, J=11.7,9.6 \mathrm{~Hz}, 1 \mathrm{H}), 3.5-3.4(\mathrm{~m}, 2 \mathrm{H}), 3.0(\mathrm{dt}, J=5.5,2.8 \mathrm{~Hz}, 1 \mathrm{H}), 2.8(\mathrm{td}, J=13.0$, $5.1 \mathrm{~Hz}, 1 \mathrm{H}$ ), $2.7(\mathrm{qd}, J=12.3,4.7 \mathrm{~Hz}, 1 \mathrm{H}), 2.4(\mathrm{ddd}, J=12.1,5.3,2.7 \mathrm{~Hz}, 1 \mathrm{H}$ ), 1.9 (ddd, $J=14.9,7.6,3.3 \mathrm{~Hz}$, 2H), $1.9-1.8(\mathrm{~m}, 1 \mathrm{H}), 1.7(\mathrm{dtt}, J=13.0,4.5,2.1 \mathrm{~Hz}, 1 \mathrm{H}), 1.3-1.2(\mathrm{~m}, 2 \mathrm{H}), 1.1(\mathrm{ddt}, J=13.2,4.1,2.2 \mathrm{~Hz}$, $1 \mathrm{H})$.

${ }^{13} \mathrm{C}$ NMR $\left(151 \mathrm{MHz}, \mathrm{CDCl}_{3}\right) \delta 174.0,168.2,161.6(\mathrm{~d}, J=244.7 \mathrm{~Hz}), 140.9,138.3(\mathrm{~d}, J=3.2 \mathrm{~Hz}), 133.8$, 132.1, 129.1 (d, $J=7.8 \mathrm{~Hz}), 123.1,116.4,115.8,66.9,51.5,45.5,40.2,35.5,29.8,26.9,25.7,23.6$.

${ }^{19} \mathrm{~F}$ NMR $\left.\left(565 \mathrm{MHz}^{\mathrm{CDCl}}\right)_{3}\right)-116.25$.

HRMS: (ESI-TOF): m/z: [M+Na] $]^{+}$Calculated for $\mathrm{C}_{28} \mathrm{H}_{29} \mathrm{FNaN}_{2} \mathrm{O}_{4}$ : 499.2004. Found: 499.2003.

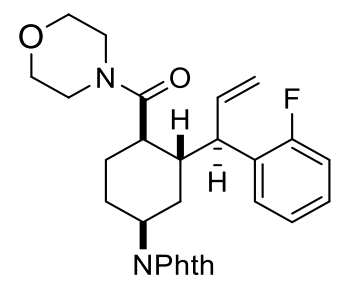

(1S,2S,4S)-4-(1,3-dioxoisoindolin-2-yl)-2-((R)-1-(2-fluorophenyl)allyl)-N-methoxy-N-methylcyclohexane1-carboxamide $(\mathbf{4 m})$

Prepared using Procedure E and G from 1g (42.5 mg, $0.1 \mathrm{mmol})$

Isolated: $23.1 \mathrm{mg}$

Yield: $50 \%$

Physical state: white solid

TLC: $R_{f}=0.23$ in 50 ethyl acetate/hexanes. Column $50 \%$ ethyl acetate hexanes

${ }^{1} \mathrm{H}$ NMR: $\left(600 \mathrm{MHz}, \mathrm{CDCl}_{3}\right) \delta 7.76(\mathrm{tt}, J=5.2,2.4 \mathrm{~Hz}, 2 \mathrm{H}), 7.68-7.63(\mathrm{~m}, 2 \mathrm{H}), 7.32(\mathrm{td}, J=7.5,1.8 \mathrm{~Hz}$, $1 \mathrm{H}), 7.17(\mathrm{dtt}, J=10.6,5.1,2.5 \mathrm{~Hz}, 1 \mathrm{H}), 7.11(\mathrm{td}, J=7.5,1.3 \mathrm{~Hz}, 1 \mathrm{H}$ ), 7.03 (ddd, $J=10.9,8.1,1.4 \mathrm{~Hz}, 1 \mathrm{H}$ ), $6.05(\mathrm{dtd}, J=17.0,9.9,2.0 \mathrm{~Hz}, 1 \mathrm{H}), 5.26(\mathrm{~d}, J=16.9 \mathrm{~Hz}, 1 \mathrm{H}), 5.16(\mathrm{ddd}, J=10.1,3.4,1.5 \mathrm{~Hz}, 1 \mathrm{H}), 4.42$ (ddt, $J=12.4,8.2,4.1 \mathrm{~Hz}, 1 \mathrm{H}), 3.86(\mathrm{dd}, J=11.7,9.7 \mathrm{~Hz}, 1 \mathrm{H}), 3.73(\mathrm{t}, J=3.9 \mathrm{~Hz}, 6 \mathrm{H}), 3.53(\mathrm{~s}, 2 \mathrm{H}), 2.99(\mathrm{p}, J$ $=2.6 \mathrm{~Hz}, 1 \mathrm{H}), 2.87-2.74(\mathrm{~m}, 2 \mathrm{H}), 2.65(\mathrm{~d}, J=11.2 \mathrm{~Hz}, 1 \mathrm{H}), 1.95(\mathrm{dq}, J=14.6,4.1 \mathrm{~Hz}, 1 \mathrm{H}), 1.89(\mathrm{tt}, J=$ $14.2,4.9 \mathrm{~Hz}, 1 \mathrm{H}), 1.79(\mathrm{~s}, 1 \mathrm{H}), 1.72-1.65(\mathrm{~m}, 1 \mathrm{H}), 1.27(\mathrm{~s}, 1 \mathrm{H}), 1.24-1.17(\mathrm{~m}, 1 \mathrm{H})$.

${ }^{13} \mathrm{C}$ NMR $\left(151 \mathrm{MHz}, \mathrm{CDCl}_{3}\right) \delta 174.0,168.2,139.1,133.8,132.2,129.8,128.3,124.7,123.2,123.1,117.40$, $116.2,116.0,47.1,45.7,38.8,35.4,27.2,25.8,23.3$.

${ }^{19}$ F NMR $\left(565 \mathrm{MHz}, \mathrm{CDCl}_{3}\right) \delta-116.7$. 
HRMS: (ESI-TOF) Calculated for $\mathrm{m} / \mathrm{z}$ : $[\mathrm{M}+\mathrm{Na}]^{+} \mathrm{C}_{28} \mathrm{H}_{29} \mathrm{FNaN}_{2} \mathrm{O}_{4}=499.2004$. Found 499.2014.<smiles>C=C[C@H](c1ccc(OC)cc1)[C@H]1C[C@H](Nc2ccccc2)CCC1C(=O)N(C)OC</smiles>

(1S,2S,4S)-4-(1,3-dioxoisoindolin-2-yl)-N-methoxy-2-((R)-1-(4-methoxyphenyl)allyl)-Nmethylcyclohexane-1-carboxamide (4n)

Prepared using Procedure E and G from 1i $(87.5 \mathrm{mg}, 0.2 \mathrm{mmol})$

Isolated: $42.1 \mathrm{mg}$

Yield: $46 \%$

Physical state: white solid

TLC: $R_{f}=0.45$ in 50\% ethyl acetate/hexanes Column: $30-50 \%$ ethyl acetate/hexanes

${ }^{1} \mathrm{H}$ NMR $\left(600 \mathrm{MHz}, \mathrm{CDCl}_{3}\right) \delta 7.73(\mathrm{dd}, J=5.4,3.1 \mathrm{~Hz}, 2 \mathrm{H}), 7.62(\mathrm{dd}, J=5.5,3.0 \mathrm{~Hz}, 2 \mathrm{H}), 7.21-7.17(\mathrm{~m}$, $2 \mathrm{H}), 6.84-6.80(\mathrm{~m}, 2 \mathrm{H}), 5.95(\mathrm{dt}, J=16.9,9.8 \mathrm{~Hz}, 1 \mathrm{H}), 5.14(\mathrm{dd}, J=17.0,1.7 \mathrm{~Hz}, 1 \mathrm{H}), 5.06(\mathrm{dd}, J=10.0$, $1.7 \mathrm{~Hz}, 1 \mathrm{H}), 4.38(\mathrm{tt}, J=12.7,4.0 \mathrm{~Hz}, 1 \mathrm{H}), 3.72(\mathrm{~s}, 3 \mathrm{H}), 3.70(\mathrm{~s}, 3 \mathrm{H}), 3.54(\mathrm{dd}, J=11.8,9.6 \mathrm{~Hz}, 1 \mathrm{H}), 3.23(\mathrm{~s}$, $3 \mathrm{H}), 2.82(\mathrm{qd}, J=12.6,4.4 \mathrm{~Hz}, 1 \mathrm{H}), 2.64(\mathrm{td}, J=13.1,5.1 \mathrm{~Hz}, 1 \mathrm{H}), 2.46-2.40(\mathrm{~m}, 1 \mathrm{H}), 2.04-1.95(\mathrm{~m}, 1 \mathrm{H})$, $1.84(\mathrm{tt}, J=13.8,4.8 \mathrm{~Hz}, 1 \mathrm{H}), 1.67-1.60(\mathrm{~m}, 1 \mathrm{H}), 1.16(\mathrm{dd}, J=10.6,6.6 \mathrm{~Hz}, 1 \mathrm{H})$

${ }^{13} \mathrm{C}$ NMR $\left(151 \mathrm{MHz}, \mathrm{CDCl}_{3}\right) \delta 168.2,158.2,141.6,135.1,133.7,132.2,128.6,115.3,114.3,114.2,61.2$, $55.3,51.0,45.7,40.3,35.6,26.8,25.9,22.7$.

HRMS: (ESI-TOF) $\mathrm{m} / \mathrm{z}$ : $[\mathrm{M}+\mathrm{H}]^{+}$Calculated for $\mathrm{C}_{27} \mathrm{H}_{31} \mathrm{~N}_{2} \mathrm{O}_{5}=463.2227$ Found $=463.2239$<smiles>C=CC(c1ccc(C2OCCO2)cc1)[C@H]1C[C@H](Nc2ccccc2)CCC1C(=O)N1CCOCC1</smiles>

2-((1S,3S,4S)-3-((R)-1-(4-(1,3-dioxolan-2-yl)phenyl)allyl)-4-(morpholine-4carbonyl)cyclohexyl)isoindoline-1,3-dione (40)

Prepared using Procedures E and G from 1j (95.9 mg, $0.2 \mathrm{mmol}$ )

Notes: Reaction was extracted with water instead of $2 \mathrm{~N}$ aq. $\mathrm{HCl}$ to avoid deprotection.

Yield: $47 \%$

Isolated: $49.8 \mathrm{mg}$ 
TLC: $R_{f}=0.13$ in $50 \%$ ethyl acetate in hexanes. Column $50-75 \%$ ethyl acetate/hexanes

${ }^{1} \mathrm{H}$ NMR $\left(600 \mathrm{MHz}, \mathrm{CDCl}_{3}\right) \delta 7.73(\mathrm{dd}, J=5.4,3.0 \mathrm{~Hz}, 2 \mathrm{H}), 7.65-7.60(\mathrm{~m}, 2 \mathrm{H}), 7.40(\mathrm{~d}, J=8.0 \mathrm{~Hz}, 2 \mathrm{H}), 7.27$ $(\mathrm{d}, J=8.0 \mathrm{~Hz}, 3 \mathrm{H}), 5.90(\mathrm{dt}, J=16.9,9.8 \mathrm{~Hz}, 1 \mathrm{H}), 5.71(\mathrm{~s}, 1 \mathrm{H}), 5.16(\mathrm{dd}, J=16.9,1.5 \mathrm{~Hz}, 1 \mathrm{H}), 5.07(\mathrm{dd}, J=$ 9.9, $1.5 \mathrm{~Hz}, 1 \mathrm{H}), 4.37(\mathrm{tt}, J=12.3,4.3 \mathrm{~Hz}, 1 \mathrm{H}), 4.13-4.04(\mathrm{~m}, 3 \mathrm{H}), 4.03-3.93(\mathrm{~m}, 3 \mathrm{H}), 3.77-3.64(\mathrm{~m}$, $4 \mathrm{H}), 3.59(\mathrm{td}, J=12.9,11.6,9.8 \mathrm{~Hz}, 2 \mathrm{H}), 3.53-3.47(\mathrm{~m}, 4 \mathrm{H}), 2.77(\mathrm{td}, J=13.0,5.2 \mathrm{~Hz}, 1 \mathrm{H}), 2.68(\mathrm{qd}, J=$ $12.3,4.7 \mathrm{~Hz}, 1 \mathrm{H}), 2.47(\mathrm{ddt}, J=11.3,5.4,2.6 \mathrm{~Hz}, 1 \mathrm{H}), 1.95-1.80(\mathrm{~m}, 2 \mathrm{H}), 1.69-1.61(\mathrm{~m}, 2 \mathrm{H}), 1.21-1.14$ $(\mathrm{m}, 1 \mathrm{H})$.

${ }^{13} \mathrm{C} \mathrm{NMR}\left(151 \mathrm{MHz}, \mathrm{CDCl}_{3}\right) \delta 17,168.1,143.7,140.8,133.7,132.0,127.7,127.1,123.0,116.3,103.4$, $67.1,66.8,65.3,65.3,65.3,52.0,45.4,40.0,35.4,26.7,25.7,23.5$.

HRMS: (ESI-TOF) m/z: [M+H] $]^{+}$Calculated for $\mathrm{C}_{31} \mathrm{H}_{35} \mathrm{~N}_{2} \mathrm{O}_{6}=531.2490$. Found 531.2466

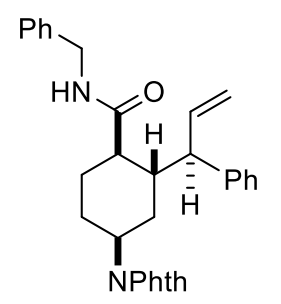

(1S,2S,4S)-N-benzyl-4-(1,3-dioxoisoindolin-2-yl)-2-((R)-1-phenylallyl)cyclohexane-1-carboxamide (5)

Prepared by General Procedure G from $3 f(15.8 \mathrm{mg}, 38.5 \mu \mathrm{mol})$

Isolated: $17.4 \mathrm{mg}$

Yield: $94 \%$ yield

Appearance: White solid

TLC: $R_{f}=0.16$ in $30 \%$ ethyl acetate/hexanes

${ }^{1} \mathrm{H}$ NMR $\left(600 \mathrm{MHz}, \mathrm{CDCl}_{3}\right) \delta 7.76(\mathrm{dd}, J=5.4,3.1 \mathrm{~Hz}, 2 \mathrm{H}), 7.68-7.62(\mathrm{~m}, 2 \mathrm{H}), 7.41-7.34(\mathrm{~m}, 4 \mathrm{H}), 7.33-$ $7.26(\mathrm{~m}, 5 \mathrm{H}), 7.16(\mathrm{tt}, J=6.4,2.2 \mathrm{~Hz}, 1 \mathrm{H}), 6.01-5.97(\mathrm{~m}, 1 \mathrm{H}), 5.97-5.93(\mathrm{~m}, 1 \mathrm{H}), 5.16(\mathrm{dd}, J=16.9,1.6$ $\mathrm{Hz}, 1 \mathrm{H}), 5.06(\mathrm{dd}, J=10.0,1.6 \mathrm{~Hz}, 1 \mathrm{H}), 4.62(\mathrm{dd}, J=14.7,5.7 \mathrm{~Hz}, 1 \mathrm{H}), 4.54(\mathrm{dd}, J=14.7,5.4 \mathrm{~Hz}, 1 \mathrm{H}), 4.44$ (tt, $J=12.7,4.1 \mathrm{~Hz}, 1 \mathrm{H}$ ), $3.56(\mathrm{dd}, J=11.7,9.6 \mathrm{~Hz}, 1 \mathrm{H}), 2.77$ (q, $J=6.6,5.6 \mathrm{~Hz}, 2 \mathrm{H}), 2.52$ (tdt, $J=12.7,9.5$, $4.3 \mathrm{~Hz}, 2 \mathrm{H}), 2.05(\mathrm{dq}, J=14.9,3.6 \mathrm{~Hz}, 1 \mathrm{H}), 2.00-1.91(\mathrm{~m}, 1 \mathrm{H}), 1.75-1.67(\mathrm{~m}, 1 \mathrm{H}), 1.19(\mathrm{ddq}, J=13.4$, 3.7, $1.8 \mathrm{~Hz}, 1 \mathrm{H})$.

${ }^{13} \mathrm{C}$ NMR $\left(151 \mathrm{MHz}, \mathrm{CDCl}_{3}\right) \delta 173.9,168.2,142.9,141.0,138.8,133.9,132.1,129.0,128.1,127.8,127.6$, $126.7,123.1,116.1,51.5,45.5,44.0,40.6,40.1,27.2,26.1,23.0$.

HRMS: (ESI-TOF) m/z: $[\mathrm{M}+\mathrm{H}]^{+}$Calculated for $\mathrm{C}_{31} \mathrm{H}_{31} \mathrm{~N}_{2} \mathrm{O}_{3}=479.2329$. Found 479.2324.

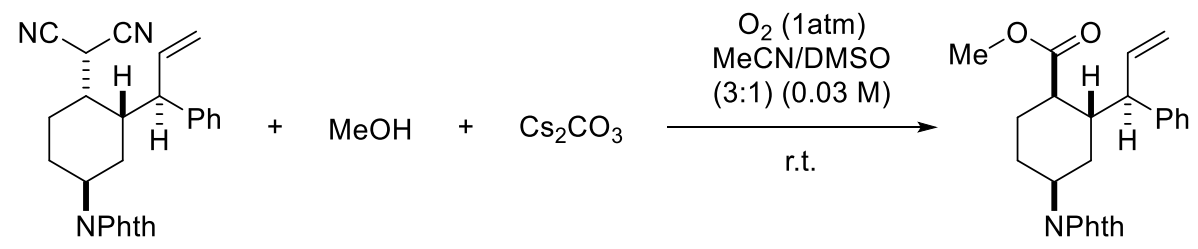


methyl (1S,2S,4S)-4-(1,3-dioxoisoindolin-2-yl)-2-((R)-1-phenylallyl)cyclohexane-1-carboxylate (6)

Adapted from Hayashi. ${ }^{10}$ The malononitrile $3 f(50 \mathrm{mg}, 0.122 \mathrm{mmol}$ ) was dissolved in in acetonitrile/DMSO (3:1) (0.03M). Methanol ( $25 \mu \mathrm{L}, 19.6 \mathrm{mg}, 061 \mathrm{mmol}, 5$ equiv.) and $\mathrm{Cs}_{2} \mathrm{CO}_{3}(199 \mathrm{mg}$, $0.61 \mathrm{mmol}, 5$ equiv.) were added. An oxygen balloon was bubbled through the reaction mixture and the reaction was stirred at room temperature overning. Once complete, the reaction mixture was vacuum filtered through a silica plug and washed with ethyl acetate. DMSO was removed by washing with $5 x$ volume of $2 \mathrm{~N}$ aq. $\mathrm{HCl}$ and back extracting with ethyl acetate. Combined organics were washed with brine, dried with $\mathrm{Na}_{2} \mathrm{SO}_{4}$, and concentrated. Crude mixture was purified using column chromatography.

Isolated: $33 \mathrm{mg}$

Yield: $67 \%$

Physical state: colorless oil

TLC: $R_{f}=0.56$ in $30 \%$ ethyl acetate/hexanes. Column $20-30 \%$ ethyl acetate/hexanes

${ }^{1}$ H NMR: $\left(600 \mathrm{MHz}, \mathrm{CDCl}_{3}\right) \delta 7.75-7.71(\mathrm{~m}, 2 \mathrm{H}), 7.64(\mathrm{td}, J=5.7,3.0 \mathrm{~Hz}, 3 \mathrm{H}), 7.37-7.29(\mathrm{~m}, 5 \mathrm{H}), 7.16$ (pt, $J=5.7,2.7 \mathrm{~Hz}, 1 \mathrm{H}$ ), $5.97(\mathrm{dt}, J=17.0,9.8 \mathrm{~Hz}, 1 \mathrm{H}$ ), 5.18 (dd, $J=17.0,1.6 \mathrm{~Hz}, 1 \mathrm{H}), 5.09$ (dd, $J=10.0,1.6$ $\mathrm{Hz}, 1 \mathrm{H}), 4.41(\mathrm{tt}, J=12.8,4.0 \mathrm{~Hz}, 1 \mathrm{H}), 3.80(\mathrm{~s}, 3 \mathrm{H}), 3.56(\mathrm{dd}, J=12.1,9.6 \mathrm{~Hz}, 1 \mathrm{H}), 2.90(\mathrm{dt}, J=6.3,3.2 \mathrm{~Hz}$, $1 \mathrm{H}), 2.42-2.28(\mathrm{~m}, 2 \mathrm{H}), 2.28-2.23(\mathrm{~m}, 1 \mathrm{H}), 2.14(\mathrm{dt}, J=14.1,3.7 \mathrm{~Hz}, \mathrm{OH}), 1.98(\mathrm{td}, J=13.5,3.9 \mathrm{~Hz}, \mathrm{OH})$, $1.94-1.88(\mathrm{~m}, \mathrm{OH}), 1.82(\mathrm{tt}, J=14.1,4.4 \mathrm{~Hz}, 1 \mathrm{H}), 1.69(\mathrm{dq}, J=12.9,3.4,2.5 \mathrm{~Hz}, 1 \mathrm{H}), 1.38-1.32(\mathrm{~m}, \mathrm{OH})$, $1.16(\mathrm{ddq}, J=13.4,3.9,2.0 \mathrm{~Hz}, 1 \mathrm{H})$.

${ }^{13} \mathrm{C}$ NMR: $\left(151 \mathrm{MHz}, \mathrm{CDCl}_{3}\right) \delta$ 175.0, 168.1, 142.7, 140.7, 133.7, 132.0, 128.9, 128.9, 126.6, 123.0, 116.1, 52.0, 51.0, 50.1, 45.3, 44.6, 39.9, 27.1, 26.2, 21.8.

HRMS: (ESI-TOF) m/z: [M+Na] ${ }^{+}$Calculated for $\mathrm{C}_{25} \mathrm{H}_{25} \mathrm{NNaO}_{4}=426.1676$. Found: 426.1667.

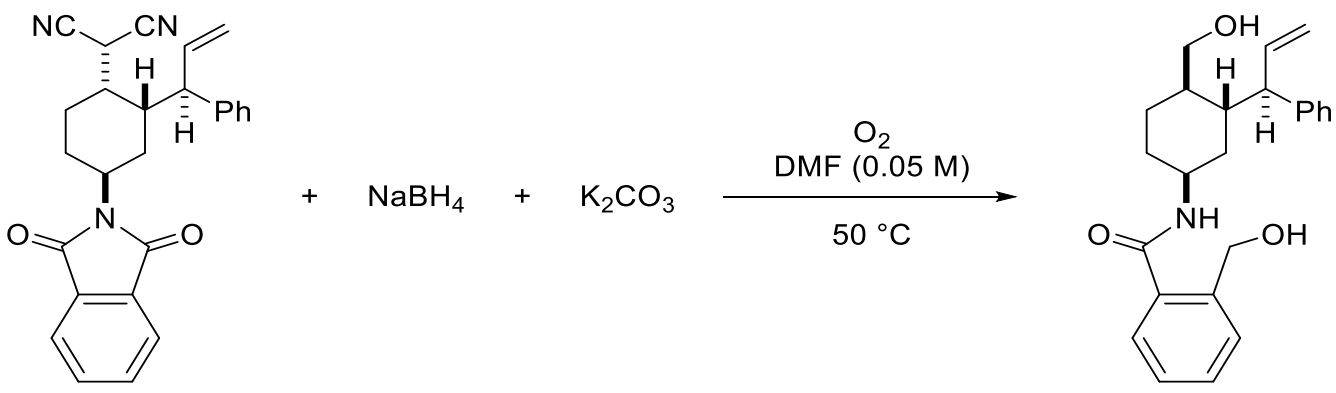

2-(hydroxymethyl)-N-((1S,3S,4R)-4-(hydroxymethyl)-3-((R)-1-phenylallyl)cyclohexyl)benzamide (7)

Procedure: Adapted from Emmetiere. ${ }^{12}$ To a flame-dried Schlenk flask was added $\mathbf{3 f}$ (19.2 mg, 0.046 mmol, >25:1 d.r.) and dry DMF (0.05 M, $1 \mathrm{~mL}$ ). The solution was freeze-pump-thaw degassed three times, backfilling with $\mathrm{N}_{2}$. 3 A Molecular sieves $(20 \mathrm{mg})$ were added and the reaction was bubbled with oxygen for 10 minutes, at which point, $\mathrm{NaBH}_{4}(3.6 \mathrm{mg}, 0.094 \mathrm{mmol})$ and $\mathrm{K}_{2} \mathrm{CO}_{3}(13 \mathrm{mg}, 0.094$ equiv.) were added. Reaction was stirred at $50{ }^{\circ} \mathrm{C}$ until complete by TLC. Reaction was quenched water ( $\left.1 \mathrm{~mL}\right)$ diluted with $2 \mathrm{M}$ aq. $\mathrm{HCl}(5 \mathrm{~mL})$. The resulting aqueous mixture was extracted with ethyl acetate $(3 \times 6$ $\mathrm{mL}$ ). The combined organic layers were washed with $2 \mathrm{M}$ aq. $\mathrm{HCl}(15 \mathrm{~mL})$ and brine (equal volume as the organic phase), and dried over $\mathrm{Na}_{2} \mathrm{SO}_{4}$. The combined organic layers were concentrated under reduced 
pressure and the crude was purified via silica gel column chromatography (25-50\% ethyl acetate/hexanes.)

isolated: $7.8 \mathrm{mg}$

Yield: $43 \%$

Appearance: white solid

TLC: $R_{f}=0.1$ in $75 \%$ ethyl acetate/hexanes.

${ }^{1} \mathbf{H}$ NMR $\left(600 \mathrm{MHz}, \mathrm{CDCl}_{3}\right) \delta 7.49-7.42(\mathrm{~m}, 2 \mathrm{H}), 7.40-7.34(\mathrm{~m}, 2 \mathrm{H}), 7.28(\mathrm{t}, J=7.6 \mathrm{~Hz}, 2 \mathrm{H}), 7.22-7.16$ $(\mathrm{m}, 1 \mathrm{H}), 7.16-7.11(\mathrm{~m}, 2 \mathrm{H}), 6.50(\mathrm{~s}, 1 \mathrm{H}), 6.02(\mathrm{dt}, J=17.0,9.8 \mathrm{~Hz}, 1 \mathrm{H}), 5.12(\mathrm{dd}, J=16.9,1.5 \mathrm{~Hz}, 1 \mathrm{H})$, $5.05(\mathrm{dd}, J=10.1,1.5 \mathrm{~Hz}, 1 \mathrm{H}), 4.51(\mathrm{~d}, J=11.8 \mathrm{~Hz}, 1 \mathrm{H}), 4.48(\mathrm{~d}, J=12.0 \mathrm{~Hz}, 1 \mathrm{H}), 4.29(\mathrm{dp}, J=8.8,4.4 \mathrm{~Hz}$, $1 \mathrm{H}), 3.90(\mathrm{dd}, J=10.5,4.9 \mathrm{~Hz}, 1 \mathrm{H}), 3.75(\mathrm{t}, J=10.0 \mathrm{~Hz}, 1 \mathrm{H}), 3.20(\mathrm{t}, J=10.4 \mathrm{~Hz}, 1 \mathrm{H}), 2.15(\mathrm{~d}, J=11.0 \mathrm{~Hz}$,

$2 \mathrm{H}), 2.00-1.88(\mathrm{~m}, 1 \mathrm{H}), 1.67-1.51(\mathrm{~m}, 5 \mathrm{H}), 1.45-1.33(\mathrm{~m}, 2 \mathrm{H}), 1.30-1.27(\mathrm{~m}, 4 \mathrm{H}), 0.88(\mathrm{t}, J=6.9 \mathrm{~Hz}$, $1 \mathrm{H})$.

${ }^{13} \mathrm{C}$ NMR $\left(151 \mathrm{MHz}, \mathrm{CDCl}_{3}\right) \delta 169.0,143.7,139.7,136.4,131.2,130.9,129.0,128.8,128.4,128.3,127.9$, $127.5,126.8,115.7,64.8,45.4,39.4,38.78,29.9,22.8,22.6,14.3$.

HRMS (DART-TOF) m/z: $[\mathrm{M}+\mathrm{H}]^{+}$Calculated for $\mathrm{C}_{24} \mathrm{H}_{30} \mathrm{NO}_{3}=380.2220$. Found 380.2335.

(ESI-TOF) $\mathrm{m} / \mathrm{z}:[\mathrm{M}+\mathrm{Na}]^{+}$Calculated for $\mathrm{C}_{24} \mathrm{H}_{29} \mathrm{NNaO}_{3}=402.2045$. Found 402.2039.

(ESI-TOF) m/z: [M-H] $]^{-}$Calculated for $\mathrm{C}_{24} \mathrm{H}_{28} \mathrm{NO}_{3}=278.2075$. Found 378.2086. 


\section{Divergent Functionalization}

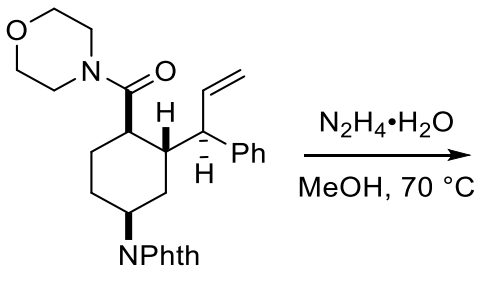

$4 \mathbf{j}$

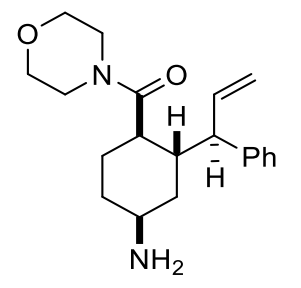

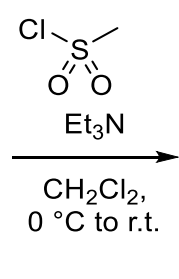

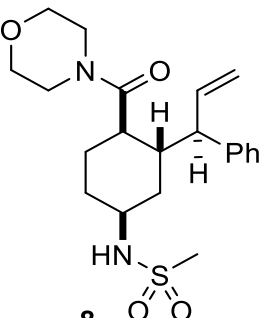

8 O

$\mathrm{N}-((1 \mathrm{~S}, 3 \mathrm{~S}, 4 \mathrm{~S})-4-($ morpholine-4-carbonyl)-3-((R)-1-phenylallyl)cyclohexyl)methanesulfonamide (8)

Adapted from Chung. ${ }^{13}$ Amide $4 \mathrm{j}$ (57.4 mg, $0.125 \mathrm{mmol}$ ) was dissolved in $\mathrm{MeOH}(0.1 \mathrm{M})$. Hydrazine hydrate ( 5 equiv. $30 \mathrm{~mL}, 0.625 \mathrm{mmol}$ ) was added and the mixture was heated at $70{ }^{\circ} \mathrm{C}$ until $\mathbf{4 j}$ disappeared by TLC ( 45 minutes). The reaction was cooled in an ice bath and $0.5 \mathrm{~mL}$ of conc. HCL was added. A white precipitate formed and was collected by vacuum filtration. The precipitate was basified with $5 \mathrm{~mL}$ of $15 \%$ aq. $\mathrm{NaOH}(\mathrm{w} / \mathrm{v})$. and extracted with ethyl acetate $(3 \times 10 \mathrm{~mL})$. Organics were washed with brine, dried with $\mathrm{Na}_{2} \mathrm{SO}_{4}$ and concentrated to yield the crude amine. The amine was dissolved in dichloromethane $(0.05 \mathrm{M})$, to which triethyl amine $(49 \mathrm{~mL}, 0.375 \mathrm{mmol}, 3$ equiv.) and the solution was cooled to $0{ }^{\circ} \mathrm{C}$. Methanesulfonyl chloride ( $43 \mathrm{~mL}, 0.5 \mathrm{mmol}, 3$ equiv.) was added and the reaction was stirred for 1 hour. Reaction was quenched with water $(10 \mathrm{~mL})$, extracted with dichloromethane $(3 \times 10$ $\mathrm{mL}$ ), washed with brine, dried with $\mathrm{Na}_{2} \mathrm{SO}_{4}$ and concentrated.

Note: 8 was observed as a 7:1 mixture of chair conformers in ${ }^{1} \mathrm{H}$ NMR. A low temperature ${ }^{1} \mathrm{H}$ NMR spectrum $\left(-50^{\circ} \mathrm{C}\right)$ showed the resolution to a single chair conformer.

Isolated: $37 \mathrm{mg}$

Yield: $73 \%$

Appearance: White solid

TLC: $R_{f}=0.21$ in ethyl acetate. Column: $50-70 \%$ ethyl acetate/hexanes to ethyl acetate.

${ }^{1} \mathrm{H}$ NMR $\left(600 \mathrm{MHz}, \mathrm{CDCl}_{3}\right) \delta 7.29(\mathrm{dt}, J=12.8,7.7 \mathrm{~Hz}, 3 \mathrm{H}), 7.25-7.18(\mathrm{~m}, 1 \mathrm{H}), 7.15-7.10(\mathrm{~m}, 2 \mathrm{H}), 7.03-$ $6.98(\mathrm{~m}, 1 \mathrm{H}), 5.99$ (ddd, $J=17.0,10.2,8.4 \mathrm{~Hz}, 1 \mathrm{H}), 5.06(\mathrm{dt}, J=17.1,1.4 \mathrm{~Hz}, 1 \mathrm{H}), 5.04-4.99(\mathrm{~m}, 1 \mathrm{H}), 4.93$ $(\mathrm{d}, J=8.1 \mathrm{~Hz}, 1 \mathrm{H}), 3.82-3.74(\mathrm{~m}, 3 \mathrm{H}), 3.73-3.67(\mathrm{~m}, 1 \mathrm{H}), 3.67-3.62(\mathrm{~m}, 1 \mathrm{H}), 3.59(\mathrm{ddd}, J=10.6,7.0$, $3.1 \mathrm{~Hz}, 1 \mathrm{H}), 3.52-3.47(\mathrm{~m}, 1 \mathrm{H}), 3.36(\mathrm{~d}, J=13.7 \mathrm{~Hz}, 1 \mathrm{H}), 3.32(\mathrm{t}, J=8.0 \mathrm{~Hz}, 3 \mathrm{H}), 2.96(\mathrm{~s}, 1 \mathrm{H}), 2.80(\mathrm{~s}, 3 \mathrm{H})$, $2.57-2.52(\mathrm{~m}, 1 \mathrm{H}), 2.35-2.28(\mathrm{~m}, 1 \mathrm{H}), 1.96-1.89(\mathrm{~m}, 1 \mathrm{H}), 1.83(\mathrm{ddd}, J=13.8,7.0,3.2 \mathrm{~Hz}, 1 \mathrm{H}), 1.67-$ $1.59(\mathrm{~m}, 1 \mathrm{H}), 1.59-1.48(\mathrm{~m}, 1 \mathrm{H}), 1.31$ (ddd, $J=14.3,11.1,3.5 \mathrm{~Hz}, 1 \mathrm{H}), 1.27-1.22(\mathrm{~m}, 1 \mathrm{H})$.

${ }^{13} \mathrm{C} \mathrm{NMR}\left(151 \mathrm{MHz}, \mathrm{CDCl}_{3}\right) \delta 173.7,141.6,140.9,129.4,128.7,128.2,126.9,126.7,115.6,67.1,66.6$, $66.6,53.8,48.7,48.5,48.5,46.0,42.2,42.2,40.79,38.3,37.1,32.5,30.6,26.1,24.5,12.6$.

HRMS: (ESI-TOF) m/z: $[\mathrm{M}+\mathrm{H}]^{+}$Calculated for $\mathrm{C}_{21} \mathrm{H}_{31} \mathrm{~N}_{2} \mathrm{O}_{4} \mathrm{~S}=407.1999$. Found 407.2000. 


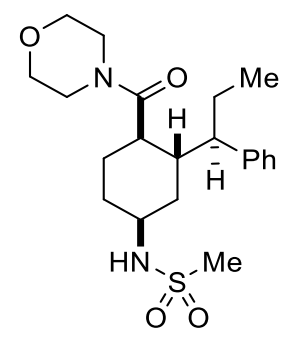

$\mathrm{N}$-((1S,3S,4S)-4-(morpholine-4-carbonyl)-3-((R)-1-phenylpropyl)cyclohexyl)methanesulfonamide (9)

To a flame-dried Schlenk flask was added $10 \%$ palladium on carbon ( $7.9 \mathrm{mg}, 3.7 \mathrm{mmol}, 10 \mathrm{~mol} \%$ ) in a glove box. Alkene 8 ( $15 \mathrm{mg}, 37 \mathrm{mmol}$ ) was added followed by anhydrous methanol ( $1 \mathrm{~mL},(0.037 \mathrm{M})$. A hydrogen balloon was added into the head space of the reaction and the reaction was stirred for 4 hours at room temperature. Upon completion, the reaction mixture was vacuum filtered through celite, washed with dichloromethane and concentrated. The product required no further purification.

Isolated: $12.4 \mathrm{mg}$

Yield: $82 \%$

Physical state: brown oil

TLC: $R_{f}=0.44$ in $100 \%$ ethyl acetate

${ }^{1} \mathrm{H}$ NMR $\left(400 \mathrm{MHz}, \mathrm{CDCl}_{3}\right) \delta 7.33-7.26(\mathrm{~m}, 2 \mathrm{H}), 7.25-7.18(\mathrm{~m}, 1 \mathrm{H}), 7.04-6.97(\mathrm{~m}, 2 \mathrm{H}), 4.93(\mathrm{~d}, J=7.7$ $\mathrm{Hz}, 1 \mathrm{H}), 3.85(\mathrm{dt}, J=7.3,3.5 \mathrm{~Hz}, 1 \mathrm{H}), 3.78(\mathrm{dq}, J=6.0,3.4,1.9 \mathrm{~Hz}, 2 \mathrm{H}), 3.74-3.62(\mathrm{~m}, 3 \mathrm{H}), 3.57$ (ddd, $J=$ $11.2,6.2,3.7 \mathrm{~Hz}, 1 \mathrm{H}), 3.25(\mathrm{dt}, J=9.4,4.4 \mathrm{~Hz}, 2 \mathrm{H}), 2.97(\mathrm{~s}, 3 \mathrm{H}), 2.75(\mathrm{dt}, J=9.8,4.7 \mathrm{~Hz}, 1 \mathrm{H}), 2.40(\mathrm{ddt}, J=$ $13.5,10.0,3.6 \mathrm{~Hz}, 1 \mathrm{H}), 2.09-2.03(\mathrm{~m}, 1 \mathrm{H}), 2.03-1.96(\mathrm{~m}, 1 \mathrm{H}), 1.81(\mathrm{dt}, J=13.8,2.7 \mathrm{~Hz}, 2 \mathrm{H}), 1.78-1.70$ $(\mathrm{m}, 1 \mathrm{H}), 1.63$ (qdd, $J=11.1,5.9,3.2 \mathrm{~Hz}, 1 \mathrm{H}), 1.52(\mathrm{dq}, J=13.7,3.9 \mathrm{~Hz}, 1 \mathrm{H}), 1.39$ (ddd, $J=17.0,8.5,3.8$ $\mathrm{Hz}, 1 \mathrm{H}), 1.35-1.27(\mathrm{~m}, 1 \mathrm{H}), 0.76(\mathrm{t}, J=7.3 \mathrm{~Hz}, 3 \mathrm{H})$.

${ }^{13} \mathrm{C}$ NMR $\left(151 \mathrm{MHz}, \mathrm{CDCl}_{3}\right) \delta 174.1,168.4,142.9,133.8,132.2,128.7,128.5,126.4,123.1,67.2,66.9$, $48.1,46.2,40.6,37.1,27.8,26.6,26.01,24.44,12.72$.

HRMS: (ESI-TOF) m/z: $[\mathrm{M}+\mathrm{H}]^{+} \mathrm{C}_{27} \mathrm{H}_{29} \mathrm{~N}_{2} \mathrm{O}_{5}=461.2071$ Found $=461.2070$<smiles>CCOC(=O)/C=C/C(=C/C=C\C(=O)C1CCC(NS(C)(=O)=O)CC1C(=O)N1CCOCC1)c1ccccc1</smiles>

ethyl (R,E)-4-((1S,2S,5S)-5-(methylsulfonamido)-2-(morpholine-4-carbonyl)cyclohexyl)-4-phenylbut-2enoate (10)

To an oven-dried 1-dram vial was added alkene $\mathbf{x x}(20 \mathrm{mg}, 0.049 \mathrm{mmol})$ and Hoveyda-Grubbs $2^{\text {nd }}$ generation catalyst (15 mol\%, $4.6 \mathrm{mg}, 0.007 \mathrm{mmol})$. The reaction was evacuated and backfilled with 
nitrogen. Anhydrous, nitrogen-sparged toluene from a solvent system was added, followed by ethyl acrylate $(19.7 \mathrm{mg}, 21 \mathrm{mmol}, 0.2 \mathrm{M})$. The reaction was capped and heated at $80^{\circ} \mathrm{C}$ for 16 hours. Upon completion the reaction was concentrated and purified by preparatory TLC (two elutions in $75 \%$ ethyl acetate/hexanes). The reaction reached $88 \%$ conversion.

Isolated: $10 \mathrm{mg}$

Yield: $42 \%$

Appearance: colorless oil

TLC: $R_{f}=0.37$ in ethyl acetate

${ }^{1} \mathrm{H}$ NMR $\left(600 \mathrm{MHz}, \mathrm{CDCl}_{3}\right) \delta 7.33(\mathrm{t}, J=7.5 \mathrm{~Hz}, 2 \mathrm{H}), 7.27(\mathrm{~d}, J=7.1 \mathrm{~Hz}, 2 \mathrm{H}), 7.14-7.12(\mathrm{~m}, 2 \mathrm{H}), 7.09$ (dd, J $=15.5,8.3 \mathrm{~Hz}, 1 \mathrm{H}), 5.80(\mathrm{dd}, J=15.5,1.2 \mathrm{~Hz}, 1 \mathrm{H}), 4.83(\mathrm{~d}, J=7.3 \mathrm{~Hz}, 1 \mathrm{H}), 4.15(\mathrm{q}, J=7.1 \mathrm{~Hz}, 2 \mathrm{H}), 3.82-$ $3.61(\mathrm{~m}, 5 \mathrm{H}), 3.57$ (ddd, $J=11.2,7.2,3.2 \mathrm{~Hz}, 1 \mathrm{H}), 3.47(\mathrm{t}, J=7.8 \mathrm{~Hz}, 2 \mathrm{H}), 3.31(\mathrm{dtq}, J=16.0,9.9,2.8 \mathrm{~Hz}$, $2 \mathrm{H}), 2.83(\mathrm{~s}, 3 \mathrm{H}), 2.66$ (dddd, $J=15.2,12.0,7.0,3.4 \mathrm{~Hz}, 1 \mathrm{H}), 2.26-2.19(\mathrm{~m}, 1 \mathrm{H}), 1.94-1.83(\mathrm{~m}, 2 \mathrm{H}), 1.74$ $-1.67(\mathrm{~m}, 1 \mathrm{H}), 1.63(\mathrm{tt}, J=13.9,3.9 \mathrm{~Hz}, 1 \mathrm{H}), 1.56-1.48(\mathrm{~m}, 1 \mathrm{H}), 1.40-1.32(\mathrm{~m}, 1 \mathrm{H}), 1.25(\mathrm{t}, J=7.1 \mathrm{~Hz}$, $3 \mathrm{H})$.

${ }^{13} \mathrm{C}$ NMR $\left(151 \mathrm{MHz}, \mathrm{CDCl}_{3}\right) \delta 173.3,166.2,150.1,139.4,128.9,128.8,127.5,121.9,66.9,66.5,60.5,52.3$, $48.3,45.8,42.2,42.0,41.3,38.1,32.6,30.3,24.24,14.3$.

HRMS: (ESI-TOF) m/z: $[\mathrm{M}+\mathrm{H}]^{+}$calculated for $\mathrm{C}_{24} \mathrm{H}_{34} \mathrm{~N}_{2} \mathrm{O}_{6} \mathrm{~S}=479.2216$, found $\mathrm{m} / \mathrm{z}:[\mathrm{M}+\mathrm{H}]=479.2203$. 


\section{Stereochemical assignment of malononitrile 3f with X-ray Crystallography}

X-Ray experimental: X-Ray Intensity data were collected at $100 \mathrm{~K}$ on a Bruker Dual micro source D8 Venture diffractometer and PHOTON III detector running APEX3 software package of programs and using MoKa radiation $(\mathrm{I}=0.71073 \AA$ ) $)$.

The data frames were integrated and multi-scan scaling was applied in APEX3. Intrinsic phasing structure solution provided the All of the non-H atoms.

The structure was refined using full-matrix least-squares refinement (SHELXL, Sheldrick G.M. 2015). ${ }^{14}$ The non- $\mathrm{H}$ atoms were refined with anisotropic displacement parameters and all of the $\mathrm{H}$ atoms were calculated in idealized positions and refined riding on their parent atoms. There are three regions of disorder in the molecules. Bothe cyanide groups are disordered and refined in two parts. The two groups on $\mathrm{C} 10$ are disordered and refined in three groups for each one. The site occupation factors for each set of disorder were refined using free variables while the three disorders on C10 refined in three part and their site occupation factors were dependently refined and were constrained to unity using the SIMP command line. In the final cycle of refinement, 4956 reflections (of which 4372 are observed with $\mathrm{I}>2 \mathrm{~s}(\mathrm{I})$ ) were used to refine 412 parameters and the resulting $\mathrm{R}_{1}, \mathrm{WR} \mathrm{R}_{2}$ and $\mathrm{S}$ (goodness of fit) were 4.45

$\%, 11.49 \%$ and 1.015 , respectively. The refinement was carried out by minimizing the $w_{2}$ function using $F^{2}$ rather than $F$ values. $R_{1}$ is calculated to provide a reference to the conventional $R$ value but its function is not minimized.
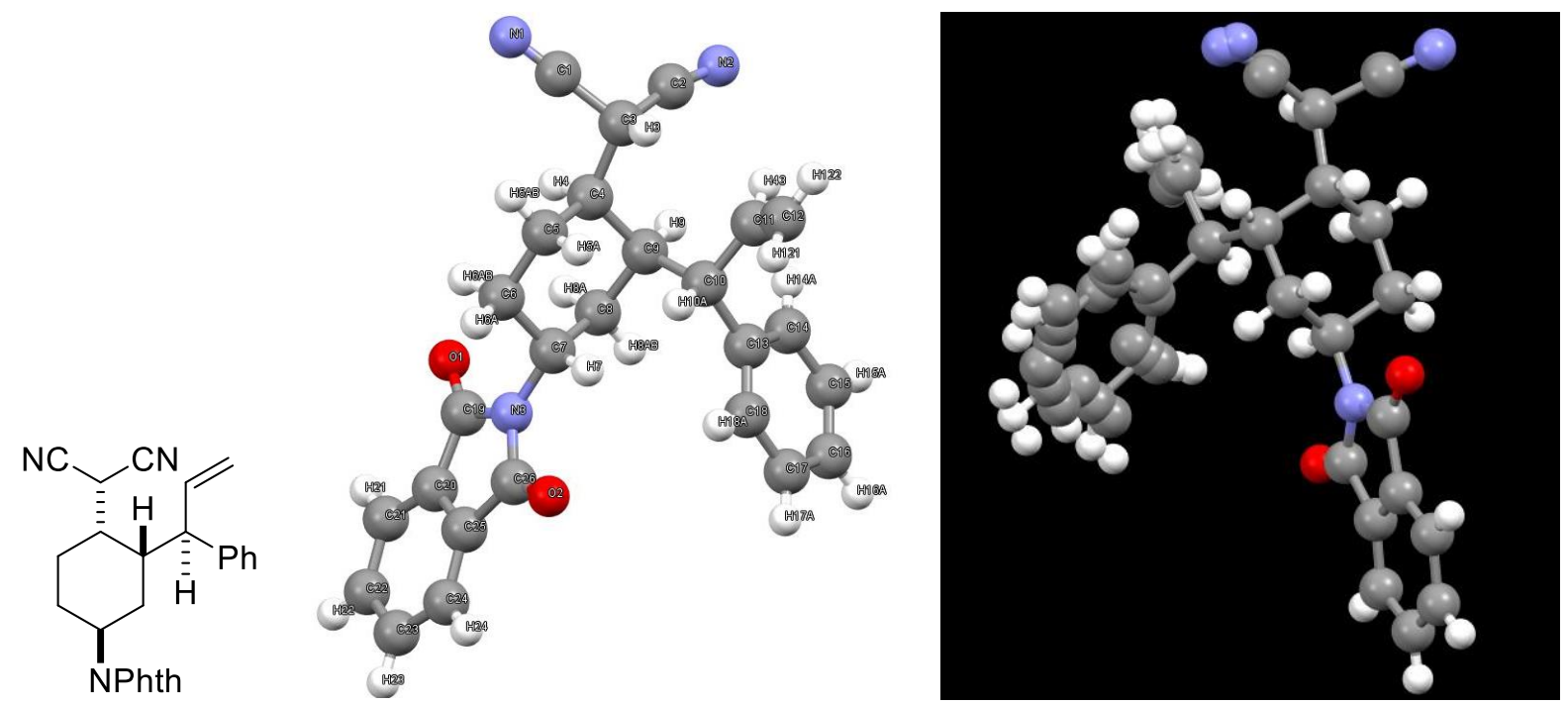

Figure S2: SHELXL refined and unrefined X-ray crystal structures of $\mathbf{3 f}$. Thermal ellipsoids probabilities are reported at $50 \%$.

Table 1. Crystal data and structure refinement for mann1.

Identification code mann1

Empirical formula $\quad \mathrm{C} 26 \mathrm{H} 23 \mathrm{~N} 3 \mathrm{O} 2$ 


$$
\begin{aligned}
& \text { Formula weight } 409.47 \\
& \text { Temperature } 100(2) \mathrm{K} \\
& \text { Wavelength } \quad 0.71073 \AA \\
& \text { Crystal system Monoclinic } \\
& \text { Space group } \quad \mathrm{P} 21 / \mathrm{c} \\
& \text { Unit cell dimensions } \quad \mathrm{a}=7.4572(3) \AA \quad \mathrm{a}=90^{\circ} . \\
& \qquad \mathrm{b}=14.9213(6) \AA \quad \mathrm{b}=91.017(2)^{\circ} . \\
& \mathrm{C}=19.3772(9) \AA \quad \mathrm{g}=90^{\circ} .
\end{aligned}
$$

Volume $2155.78(16) \AA ̊ 3$

Z $\quad 4$

Density (calculated) $\quad 1.262 \mathrm{Mg} / \mathrm{m3}$

Absorption coefficient $\quad 0.081 \mathrm{~mm}-1$

$F(000) \quad 864$

Crystal size $\quad 0.150 \times 0.110 \times 0.110 \mathrm{~mm} 3$

Theta range for data collection 2.102 to $27.499^{\circ}$.

Index ranges $\quad-9 \leq h \leq 9,-19 \leq k \leq 19,-25 \leq 1 \leq 25$

Reflections collected $\quad 69083$

Independent reflections $4956[\mathrm{R}(\mathrm{int})=0.0390]$

Completeness to theta $=25.242^{\circ} \quad 99.9 \%$

Absorption correction "Multi-Scan"

Refinement method Full-matrix least-squares on F2

Data / restraints / parameters 4956/1207 / 412

Goodness-of-fit on F2 1.015

Final $R$ indices $[\mathrm{I}>2 \operatorname{sigma}(\mathrm{I})] \quad \mathrm{R} 1=0.0445, \mathrm{wR} 2=0.1149$ [4372]

$\mathrm{R}$ indices (all data) $\quad \mathrm{R} 1=0.0498, \mathrm{wR} 2=0.1198$

Extinction coefficient $\quad n / a$

Largest diff. peak and hole $\quad 0.466$ and -0.251 e. $\AA$ - -3

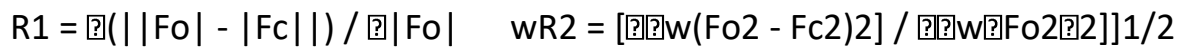


$S=[$ []?] $w(F o 2-F c 2) 2] /(n-p)] 1 / 2 w=1 /\left[\right.$ [?2 $\left.2(F o 2)+(m * p)^{2}+n * p\right], p=m / z:[M a x(F o 2,0)+2 * F c 2] / 3, m \& n$ are constants.

Table 2. Atomic coordinates ( x 104) and equivalent isotropic displacement parameters ( $\AA 2$ x 103) for mann1. $U(e q)$ is defined as one third of the trace of the orthogonalized Uij tensor.

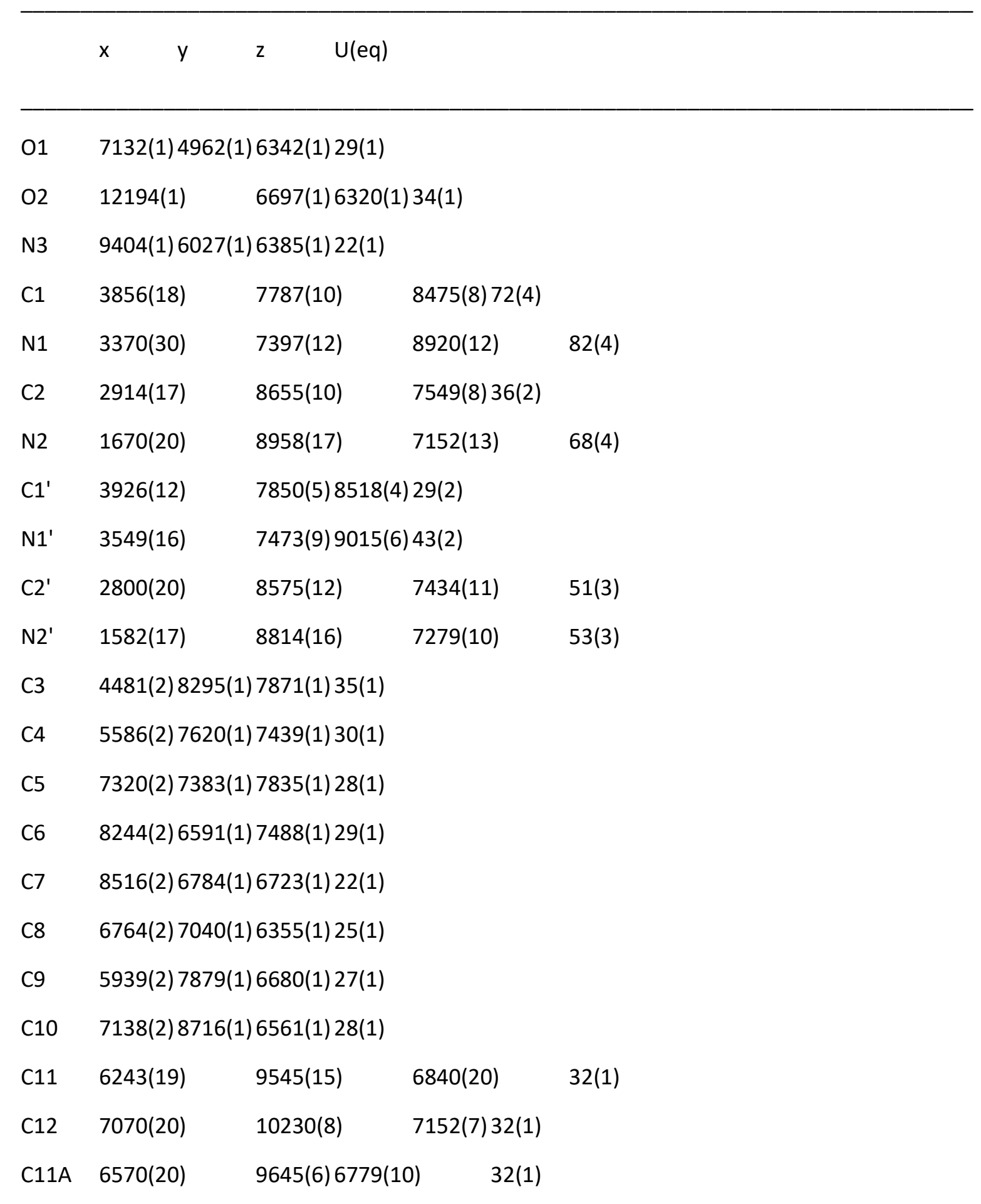




\begin{tabular}{|c|c|c|c|c|}
\hline $\mathrm{C} 12 \mathrm{~A}$ & $7460(20)$ & 10128(9) & $7248(9) 32(1)$ & \\
\hline C11B & $6259(12)$ & $9561(6) 6821(8)$ & $32(1)$ & \\
\hline $\mathrm{C} 12 \mathrm{~B}$ & 7050(11) & $10086(4)$ & $7290(4) 32(1)$ & \\
\hline C13 & $7670(70)$ & $8810(40)$ & $5802(7) 32(1)$ & \\
\hline C14 & $6306(17)$ & $8940(9) 5310(4)$ & $36(2)$ & \\
\hline C15 & $6746(18)$ & $9053(6) 4607(4)$ & $37(2)$ & \\
\hline C16 & $8500(20)$ & $9035(5) 4414(4)$ & $35(2)$ & \\
\hline C17 & $9848(16)$ & $8884(6) 4904(5)$ & ) $34(2)$ & \\
\hline $\mathrm{C} 18$ & $9381(16)$ & $8782(10)$ & $5600(7) 33(2)$ & \\
\hline C13A & $7310(20)$ & $8846(14)$ & $5779(2) 32(1)$ & \\
\hline C14A & $5830(20)$ & $8900(14)$ & $5336(5) 39(3)$ & \\
\hline C15A & $6070(30)$ & $9022(13)$ & $4632(4) 36(3)$ & \\
\hline C16A & $7790(30)$ & $9090(13)$ & $4371(2) 35(4)$ & \\
\hline C17A & $9270(20)$ & $9037(13)$ & $4814(6) 33(3)$ & \\
\hline C18A & $9030(20)$ & $8915(13)$ & $5518(5) 36(3)$ & \\
\hline C13B & $7670(160)$ & $8810(100)$ & $5802(18)$ & $32(1)$ \\
\hline C14B & $6640(40)$ & $9020(20)$ & $5195(12)$ & $42(3)$ \\
\hline C15B & $7370(30)$ & $9080(20)$ & $4575(11)$ & $38(4)$ \\
\hline C16B & $9130(30)$ & 8937(17) & $4450(11)$ & $35(3)$ \\
\hline C17B & $10310(30)$ & $8733(17)$ & $4988(10)$ & $32(3)$ \\
\hline C18B & $9620(30)$ & $8670(20)$ & $5657(15)$ & $26(3)$ \\
\hline C19 & $8655(2) 5191(1)$ & $6227(1) 23(1)$ & & \\
\hline $\mathrm{C} 20$ & $10109(2)$ & $4658(1) 5911(1)$ & $22(1)$ & \\
\hline C21 & $10106(2)$ & $3789(1) 5660(1)$ & ) $27(1)$ & \\
\hline $\mathrm{C} 22$ & $11716(2)$ & $3456(1) 5412(1)$ & ) $30(1)$ & \\
\hline $\mathrm{C} 23$ & $13255(2)$ & $3979(1) 5412(1)$ & ) $31(1)$ & \\
\hline C24 & $13247(2)$ & $4856(1) 5664(1)$ & ) $29(1)$ & \\
\hline $\mathrm{C} 25$ & $11648(2)$ & $5178(1) 5910(1)$ & 23(1) & \\
\hline $\mathrm{C} 26$ & $11220(2)$ & $6063(1) 6216(1)$ & ) 24(1) & \\
\hline
\end{tabular}


Table 3. Bond lengths $[\AA ̊]$ and angles $\left[^{\circ}\right]$ for mann1.

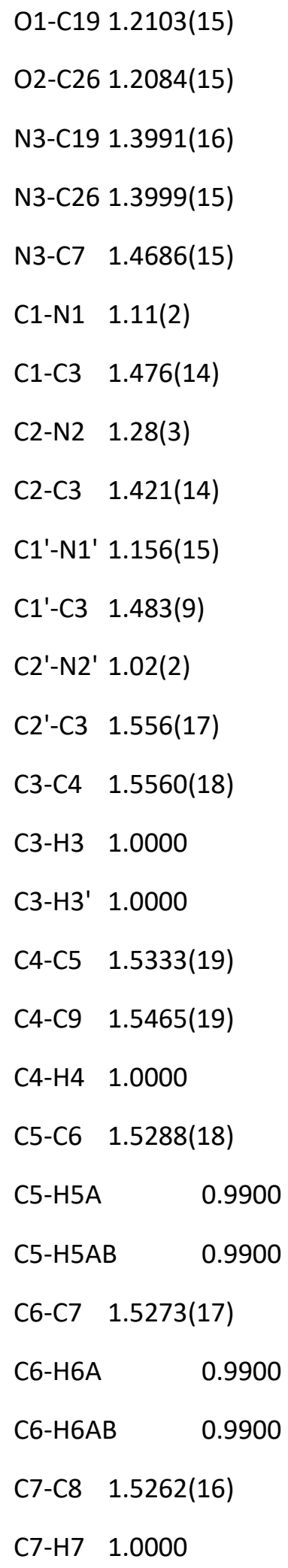




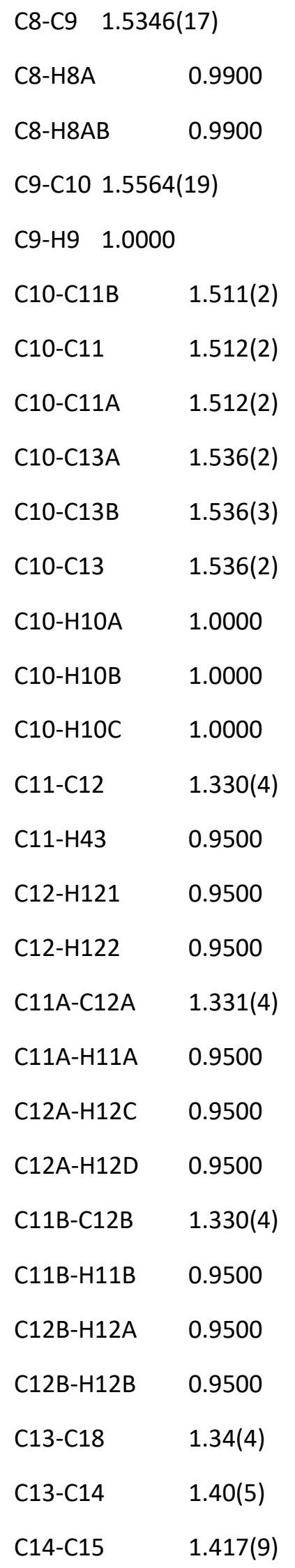




\begin{tabular}{|c|c|}
\hline $\mathrm{C} 14-\mathrm{H} 14 \mathrm{~A}$ & 0.9500 \\
\hline C15-C16 & $1.370(7)$ \\
\hline $\mathrm{C} 15-\mathrm{H} 15 \mathrm{~A}$ & 0.9500 \\
\hline C16-C17 & $1.387(6)$ \\
\hline $\mathrm{C} 16-\mathrm{H} 16 \mathrm{~A}$ & 0.9500 \\
\hline C17-C18 & $1.406(13)$ \\
\hline C17-H17A & 0.9500 \\
\hline C18-H18A & 0.9500 \\
\hline C13A-C14A & 1.3900 \\
\hline C13A-C18A & 1.3900 \\
\hline C14A-C15A & 1.3900 \\
\hline $\mathrm{C} 14 \mathrm{~A}-\mathrm{H} 14 \mathrm{~B}$ & 0.9500 \\
\hline C15A-C16A & 1.3900 \\
\hline C15A-H15B & 0.9500 \\
\hline C16A-C17A & 1.3900 \\
\hline $\mathrm{C} 16 \mathrm{~A}-\mathrm{H} 16 \mathrm{~B}$ & 0.9500 \\
\hline C17A-C18A & 1.3900 \\
\hline C17A-H17B & 0.9500 \\
\hline $\mathrm{C} 18 \mathrm{~A}-\mathrm{H} 18 \mathrm{~B}$ & 0.9500 \\
\hline C13B-C14B & $1.43(10)$ \\
\hline C13B-C18B & $1.50(12)$ \\
\hline C14B-C15B & $1.33(3)$ \\
\hline C14B-H14C & 0.9500 \\
\hline C15B-C16B & $1.356(18)$ \\
\hline C15B-H15C & 0.9500 \\
\hline C16B-C17B & $1.388(15)$ \\
\hline C16B-H16C & 0.9500 \\
\hline C17B-C18B & $1.41(3)$ \\
\hline C17B-H17C & 0.9500 \\
\hline
\end{tabular}




\begin{tabular}{|c|c|}
\hline C18B-H18C & 0.9500 \\
\hline C19-C20 & $1.4859(17)$ \\
\hline C20-C21 & $1.3844(17)$ \\
\hline $\mathrm{C} 20-\mathrm{C} 25$ & $1.3856(17)$ \\
\hline C21-C22 & $1.3921(18)$ \\
\hline $\mathrm{C} 21-\mathrm{H} 21$ & 0.9500 \\
\hline $\mathrm{C} 22-\mathrm{C} 23$ & $1.388(2)$ \\
\hline $\mathrm{C} 22-\mathrm{H} 22$ & 0.9500 \\
\hline C23-C24 & $1.3962(19)$ \\
\hline $\mathrm{C} 23-\mathrm{H} 23$ & 0.9500 \\
\hline C24-C25 & $1.3792(17)$ \\
\hline $\mathrm{C} 24-\mathrm{H} 24$ & 0.9500 \\
\hline $\mathrm{C} 25-\mathrm{C} 26$ & $1.4835(17)$ \\
\hline C19-N3-C26 & $111.48(10)$ \\
\hline C19-N3-C7 & $126.99(10)$ \\
\hline C26-N3-C7 & $121.43(10)$ \\
\hline N1-C1-C3 & $178.8(15)$ \\
\hline $\mathrm{N} 2-\mathrm{C} 2-\mathrm{C} 3$ & $169.0(19)$ \\
\hline $\mathrm{N} 1{ }^{\prime}-\mathrm{C} 11^{\prime}-\mathrm{C} 3$ & $176.9(8)$ \\
\hline $\mathrm{N} 2{ }^{\prime}-\mathrm{C} 2 '-\mathrm{C} 3$ & $164(3)$ \\
\hline $\mathrm{C} 2{ }^{\prime}-\mathrm{C} 3-\mathrm{C} 1^{\prime}$ & $110.3(7)$ \\
\hline C1-C3-C2 & 105.9(8) \\
\hline $\mathrm{C} 2$ '-C3-C4 & $108.0(8)$ \\
\hline $\mathrm{C} 1^{\prime}-\mathrm{C} 3-\mathrm{C} 4$ & $108.9(3)$ \\
\hline C1-C3-C4 & $105.8(6)$ \\
\hline $\mathrm{C} 2-\mathrm{C} 3-\mathrm{C} 4$ & 116.6(6) \\
\hline $\mathrm{C} 1-\mathrm{C} 3-\mathrm{H} 3$ & 109.4 \\
\hline $\mathrm{C} 2-\mathrm{C} 3-\mathrm{H} 3$ & 109.4 \\
\hline
\end{tabular}




\begin{tabular}{|c|c|}
\hline $\mathrm{C} 4-\mathrm{C} 3-\mathrm{H} 3$ & 109.4 \\
\hline C2'-C3-H3' & 109.9 \\
\hline C1'-C3-H3' & 109.9 \\
\hline C4-C3-H3' & 109.9 \\
\hline C5-C4-C9 & $112.15(10)$ \\
\hline $\mathrm{C} 5-\mathrm{C} 4-\mathrm{C} 3$ & $109.22(11)$ \\
\hline $\mathrm{C} 9-\mathrm{C} 4-\mathrm{C} 3$ & $116.80(12)$ \\
\hline $\mathrm{C} 5-\mathrm{C} 4-\mathrm{H} 4$ & 106.0 \\
\hline $\mathrm{C} 9-\mathrm{C} 4-\mathrm{H} 4$ & 106.0 \\
\hline $\mathrm{C} 3-\mathrm{C} 4-\mathrm{H} 4$ & 106.0 \\
\hline C6-C5-C4 & 109.94(11) \\
\hline C6-C5-H5A & 109.7 \\
\hline C4-C5-H5A & 109.7 \\
\hline $\mathrm{C} 6-\mathrm{C} 5-\mathrm{H} 5 \mathrm{AB}$ & 109.7 \\
\hline $\mathrm{C} 4-\mathrm{C} 5-\mathrm{H} 5 \mathrm{AB}$ & 109.7 \\
\hline $\mathrm{H} 5 \mathrm{~A}-\mathrm{C} 5-\mathrm{H} 5 \mathrm{AB}$ & 108.2 \\
\hline C7-C6-C5 & $110.45(10)$ \\
\hline C7-C6-H6A & 109.6 \\
\hline C5-C6-H6A & 109.6 \\
\hline C7-C6-H6AB & 109.6 \\
\hline C5-C6-H6AB & 109.6 \\
\hline H6A-C6-H6AB & 108.1 \\
\hline N3-C7-C8 & $111.92(10)$ \\
\hline N3-C7-C6 & $110.95(10)$ \\
\hline $\mathrm{C} 8-\mathrm{C} 7-\mathrm{C} 6$ & 111.94(10) \\
\hline N3-C7-H7 & 107.2 \\
\hline $\mathrm{C} 8-\mathrm{C} 7-\mathrm{H} 7$ & 107.2 \\
\hline $\mathrm{C} 6-\mathrm{C} 7-\mathrm{H} 7$ & 107.2 \\
\hline $\mathrm{C} 7-\mathrm{C} 8-\mathrm{C9}$ & $111.03(10)$ \\
\hline
\end{tabular}




\begin{tabular}{ll} 
C7-C8-H8A & 109.4 \\
C9-C8-H8A & 109.4 \\
C7-C8-H8AB & 109.4 \\
C9-C8-H8AB & 109.4 \\
H8A-C8-H8AB & 108.0 \\
C8-C9-C4 & $105.21(10)$ \\
C8-C9-C10 & $110.98(10)$ \\
C4-C9-C10 & $116.74(11)$ \\
C8-C9-H9 & 107.9 \\
C4-C9-H9 & 107.9 \\
C10-C9-H9 & 107.9 \\
C11A-C10-C13A $100.9(11)$ \\
C11B-C10-C13B $111(6)$ \\
C11-C10-C13 & $113(3)$ \\
C13B-C10-H10C & 107.2 \\
C11B-C10-C9 & $111.5(6)$ \\
C11-C10-C9 & $110.1(12)$ \\
C13A-C10-H10B & \\
C11A-C10-C9 & $122.0(7)$ \\
C13A-C10-C9 & $107.9(8)$ \\
C13B-C10-C9 & $112(5)$ \\
C13-C10-C9 & $112(2)$ \\
C11-C10-H10A & 107.0 \\
C13-C10-H10A & 107.0 \\
\hline & 108.4 \\
C10-H10A & 107.0 \\
C10C 107.2 \\
C10-H10B108.4 \\
C10
\end{tabular}




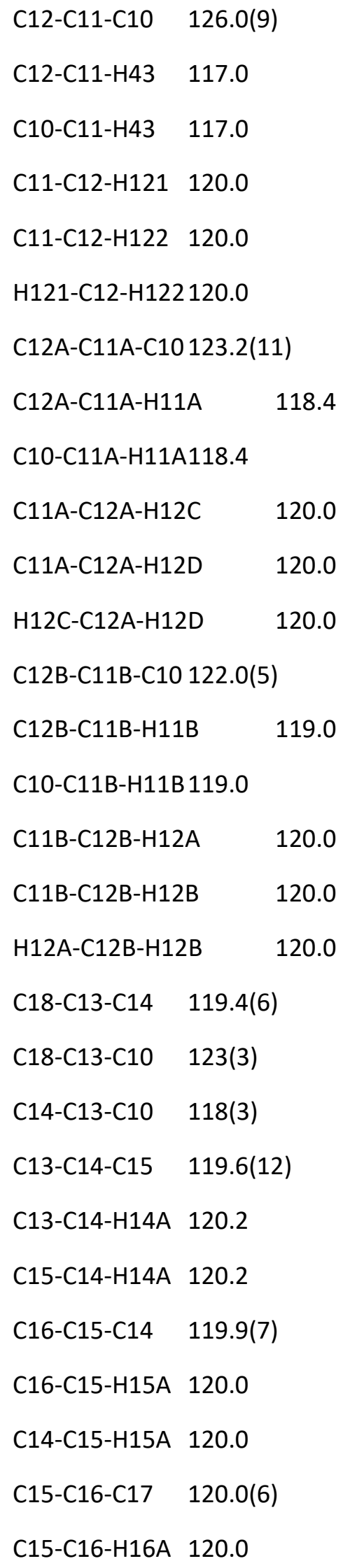




\begin{tabular}{|c|c|c|}
\hline C17-C16-H16A & 120.0 & \\
\hline C16-C17-C18 & $119.1(7$ & \\
\hline C16-C17-H17A & 120.5 & \\
\hline C18-C17-H17A & 120.5 & \\
\hline $\mathrm{C} 13-\mathrm{C} 18-\mathrm{C} 17$ & $122.0(1$ & \\
\hline C13-C18-H18A & 119.0 & \\
\hline C17-C18-H18A & 119.0 & \\
\hline $\mathrm{C} 14 \mathrm{~A}-\mathrm{C} 13 \mathrm{~A}-\mathrm{C} 18$ & & 120.0 \\
\hline $\mathrm{C} 14 \mathrm{~A}-\mathrm{C} 13 \mathrm{~A}-\mathrm{C} 10$ & $122.4(9$ & \\
\hline C18A-C13A-C10 & $117.6(9$ & \\
\hline $\mathrm{C} 13 \mathrm{~A}-\mathrm{C} 14 \mathrm{~A}-\mathrm{C} 15$ & & 120.0 \\
\hline $\mathrm{C} 13 \mathrm{~A}-\mathrm{C} 14 \mathrm{~A}-\mathrm{H} 14$ & & 120.0 \\
\hline $\mathrm{C} 15 \mathrm{~A}-\mathrm{C} 14 \mathrm{~A}-\mathrm{H} 14$ & & 120.0 \\
\hline C16A-C15A-C14 & & 120.0 \\
\hline C16A-C15A-H15 & & 120.0 \\
\hline $\mathrm{C} 14 \mathrm{~A}-\mathrm{C} 15 \mathrm{~A}-\mathrm{H} 15$ & & 120.0 \\
\hline $\mathrm{C} 15 \mathrm{~A}-\mathrm{C} 16 \mathrm{~A}-\mathrm{C} 17$ & & 120.0 \\
\hline $\mathrm{C} 15 \mathrm{~A}-\mathrm{C} 16 \mathrm{~A}-\mathrm{H} 16$ & & 120.0 \\
\hline $\mathrm{C} 17 \mathrm{~A}-\mathrm{C} 16 \mathrm{~A}-\mathrm{H} 16$ & & 120.0 \\
\hline C18A-C17A-C16 & & 120.0 \\
\hline $\mathrm{C} 18 \mathrm{~A}-\mathrm{C} 17 \mathrm{~A}-\mathrm{H} 17$ & & 120.0 \\
\hline C16A-C17A-H17 & & 120.0 \\
\hline C17A-C18A-C13 & & 120.0 \\
\hline $\mathrm{C} 17 \mathrm{~A}-\mathrm{C} 18 \mathrm{~A}-\mathrm{H} 18$ & & 120.0 \\
\hline $\mathrm{C} 13 \mathrm{~A}-\mathrm{C} 18 \mathrm{~A}-\mathrm{H} 18$ & & 120.0 \\
\hline $\mathrm{C} 14 \mathrm{~B}-\mathrm{C} 13 \mathrm{~B}-\mathrm{C} 18 \mathrm{~B}$ & & $112.6(19)$ \\
\hline C14B-C13B-C10 & $132(8)$ & \\
\hline C18B-C13B-C10 & $116(7)$ & \\
\hline C15B-C14B-C13E & & $123(4)$ \\
\hline
\end{tabular}




\begin{tabular}{|c|c|c|}
\hline \multicolumn{2}{|l|}{ C15B-C } & 118.7 \\
\hline \multicolumn{2}{|c|}{ C13B-C14B-H14C } & 118.7 \\
\hline \multicolumn{2}{|c|}{ C14B-C15B-C16B } & $124(2)$ \\
\hline \multicolumn{2}{|c|}{ C14B-C15B-H15C } & 118.0 \\
\hline \multicolumn{2}{|c|}{ C16B-C15B-H15C } & 118.0 \\
\hline \multicolumn{2}{|c|}{ C15B-C16B-C17B } & $120.5(17)$ \\
\hline \multicolumn{2}{|c|}{ C15B-C16B-H16C } & 119.8 \\
\hline \multicolumn{2}{|c|}{ C17B-C16B-H16C } & 119.8 \\
\hline \multicolumn{2}{|c|}{ C16B-C17B-C18B } & 117.9(17) \\
\hline \multicolumn{2}{|c|}{ C16B-C17B-H17C } & 121.1 \\
\hline \multicolumn{2}{|c|}{ C18B-C17B-H17C } & 121.1 \\
\hline \multicolumn{2}{|c|}{ C17B-C18B-C13B } & $122(3)$ \\
\hline \multicolumn{2}{|c|}{ C17B-C18B-H18C } & 118.8 \\
\hline \multicolumn{2}{|c|}{ C13B-C18B-H18C } & 118.8 \\
\hline O1-C19-N3 & $125.68(1)$ & \\
\hline O1-C19-C20 & $128.24(1)$ & \\
\hline N3-C19-C20 & $106.07(1$ & \\
\hline C21-C20-C25 & $121.41(1$ & \\
\hline C21-C20-C19 & $130.50(1$ & \\
\hline C25-C20-C19 & $108.08(1$ & \\
\hline $\mathrm{C} 20-\mathrm{C} 21-\mathrm{C} 22$ & $117.28(1)$ & \\
\hline $\mathrm{C} 20-\mathrm{C} 21-\mathrm{H} 21$ & 121.4 & \\
\hline $\mathrm{C} 22-\mathrm{C} 21-\mathrm{H} 21$ & 121.4 & \\
\hline C23-C22-C21 & $121.22(1$ & \\
\hline $\mathrm{C} 23-\mathrm{C} 22-\mathrm{H} 22$ & 119.4 & \\
\hline $\mathrm{C} 21-\mathrm{C} 22-\mathrm{H} 22$ & 119.4 & \\
\hline C22-C23-C24 & $121.17(1$ & \\
\hline $\mathrm{C} 22-\mathrm{C} 23-\mathrm{H} 23$ & 119.4 & \\
\hline $\mathrm{C} 24-\mathrm{C} 23-\mathrm{H} 23$ & 119.4 & \\
\hline
\end{tabular}




$\begin{array}{ll}\mathrm{C} 25-\mathrm{C} 24-\mathrm{C} 23 & 117.18(12) \\ \mathrm{C} 25-\mathrm{C} 24-\mathrm{H} 24 & 121.4 \\ \mathrm{C} 23-\mathrm{C} 24-\mathrm{H} 24 & 121.4 \\ \mathrm{C} 24-\mathrm{C} 25-\mathrm{C} 20 & 121.74(12) \\ \mathrm{C} 24-\mathrm{C} 25-\mathrm{C} 26 & 129.92(12) \\ \mathrm{C} 20-\mathrm{C} 25-\mathrm{C} 26 & 108.33(10) \\ \text { O2-C26-N3 } & 124.88(11) \\ \text { O2-C26-C25 } & 129.09(11) \\ \text { N3-C26-C25 } & 106.01(10)\end{array}$

Symmetry transformations used to generate equivalent atoms: 
Table 4. Anisotropic displacement parameters (Å2x 103) for mann1. The anisotropic displacement factor exponent takes the form: $-2 p 2[h 2 a * 2 U 11+\ldots+2 h k a * b * U 12$ ]

$\begin{array}{llllll}\text { U11 } & \text { U22 } & \text { U33 } & \text { U23 } & \text { U13 } & \text { U12 }\end{array}$

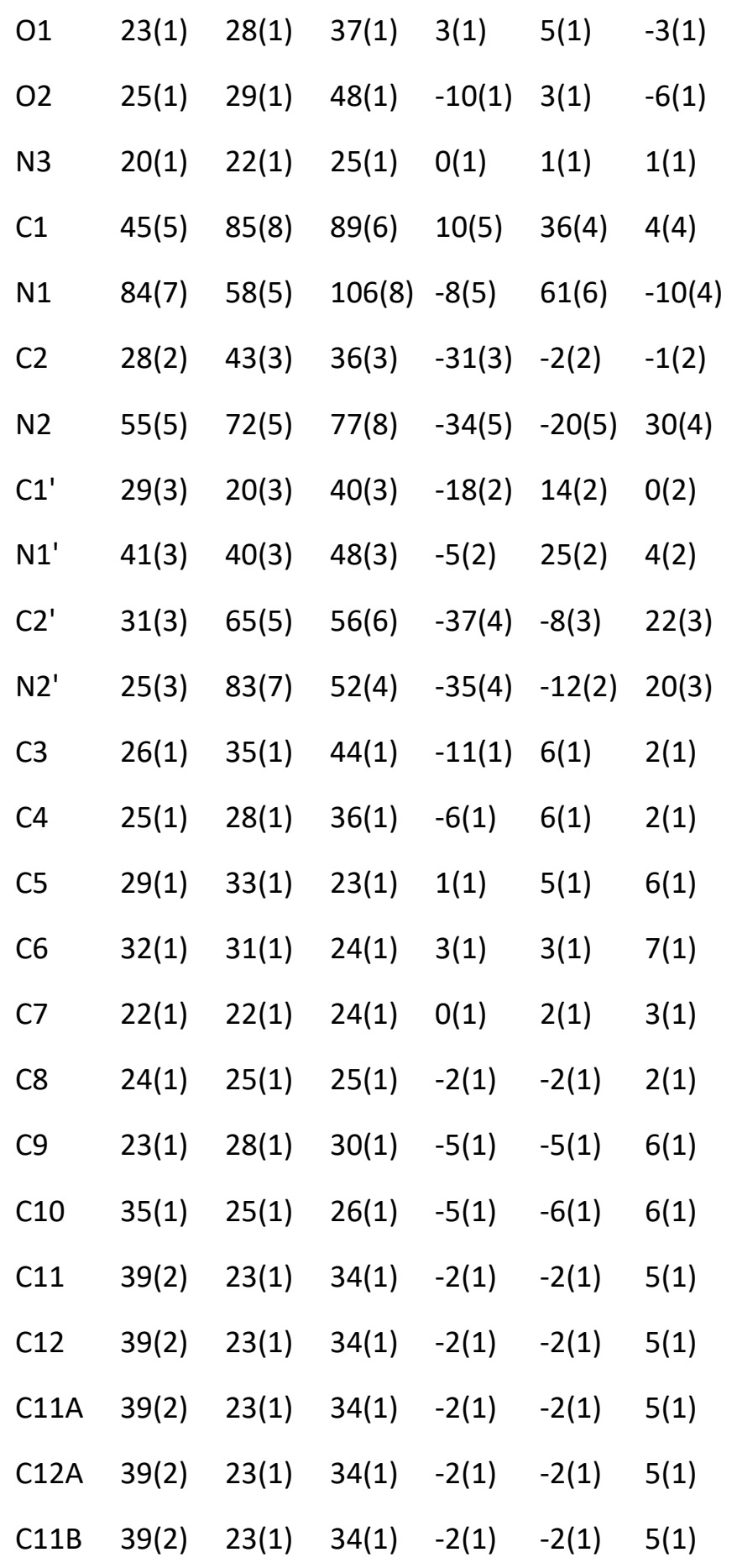




\begin{tabular}{|c|c|c|c|c|c|c|}
\hline C12B & $39(2)$ & $23(1)$ & $34(1)$ & $-2(1)$ & $-2(1)$ & $5(1)$ \\
\hline C13 & $52(2)$ & $17(1)$ & $26(1)$ & $-2(1)$ & $-8(1)$ & $3(1)$ \\
\hline C14 & $56(4)$ & $26(3)$ & $26(3)$ & $0(2)$ & $-6(2)$ & $8(3)$ \\
\hline C15 & $52(6)$ & $26(2)$ & $31(2)$ & $0(2)$ & $-17(3)$ & $5(5)$ \\
\hline C16 & $63(7)$ & $22(3)$ & $20(2)$ & $2(2)$ & $-10(3)$ & $-4(5)$ \\
\hline C17 & $54(6)$ & $22(5)$ & 24(3) & $5(2)$ & $-3(3)$ & $-3(4)$ \\
\hline C18 & $55(4)$ & $21(5)$ & $21(2)$ & $5(2)$ & $-6(2)$ & $-1(3)$ \\
\hline C13A & $52(2)$ & $17(1)$ & $26(1)$ & $-2(1)$ & $-8(1)$ & $3(1)$ \\
\hline C14A & $57(6)$ & $35(6)$ & $24(4)$ & $2(4)$ & $-18(3)$ & $16(5)$ \\
\hline C15A & $50(7)$ & $25(4)$ & $32(4)$ & $1(3)$ & $-18(4)$ & $5(6)$ \\
\hline C16A & $56(8)$ & $26(4)$ & $22(5)$ & $-3(4)$ & $-15(4)$ & $1(7)$ \\
\hline C17A & $53(7)$ & $19(5)$ & $25(4)$ & $5(4)$ & $-7(4)$ & $-3(5)$ \\
\hline C18A & $63(6)$ & $19(6)$ & $27(4)$ & $8(3)$ & $-13(4)$ & $-14(5)$ \\
\hline C13B & $52(2)$ & $17(1)$ & $26(1)$ & $-2(1)$ & $-8(1)$ & $3(1)$ \\
\hline C14B & $59(7)$ & $31(6)$ & $37(6)$ & $2(5)$ & $-5(4)$ & $15(6)$ \\
\hline C15B & $51(8)$ & $34(5)$ & $30(5)$ & $-2(5)$ & $-13(6)$ & $5(8)$ \\
\hline C16B & $52(8)$ & $27(6)$ & $24(5)$ & $-5(4)$ & $-13(5)$ & $2(6)$ \\
\hline C17B & $50(7)$ & $22(7)$ & $23(5)$ & $1(4)$ & $-10(4)$ & $1(5)$ \\
\hline C18B & $40(5)$ & $14(6)$ & $22(5)$ & $-1(4)$ & $3(4)$ & $-9(5)$ \\
\hline C19 & $24(1)$ & $22(1)$ & $22(1)$ & $4(1)$ & $0(1)$ & $0(1)$ \\
\hline C20 & $24(1)$ & $23(1)$ & $19(1)$ & $3(1)$ & $0(1)$ & $0(1)$ \\
\hline C21 & $33(1)$ & $23(1)$ & $25(1)$ & $1(1)$ & $2(1)$ & $-4(1)$ \\
\hline C22 & $41(1)$ & $23(1)$ & $24(1)$ & $-1(1)$ & $4(1)$ & $2(1)$ \\
\hline $\mathrm{C} 23$ & $30(1)$ & $33(1)$ & $29(1)$ & $-4(1)$ & $4(1)$ & $6(1)$ \\
\hline C24 & $23(1)$ & $33(1)$ & $30(1)$ & $-4(1)$ & $1(1)$ & $0(1)$ \\
\hline C25 & $24(1)$ & $24(1)$ & $21(1)$ & $0(1)$ & $-2(1)$ & $0(1)$ \\
\hline C26 & $21(1)$ & $26(1)$ & $25(1)$ & $-1(1)$ & $0(1)$ & $1(1)$ \\
\hline
\end{tabular}


Table 5. Hydrogen coordinates ( x 104) and isotropic displacement parameters (Å2x 103 )

for mann1.

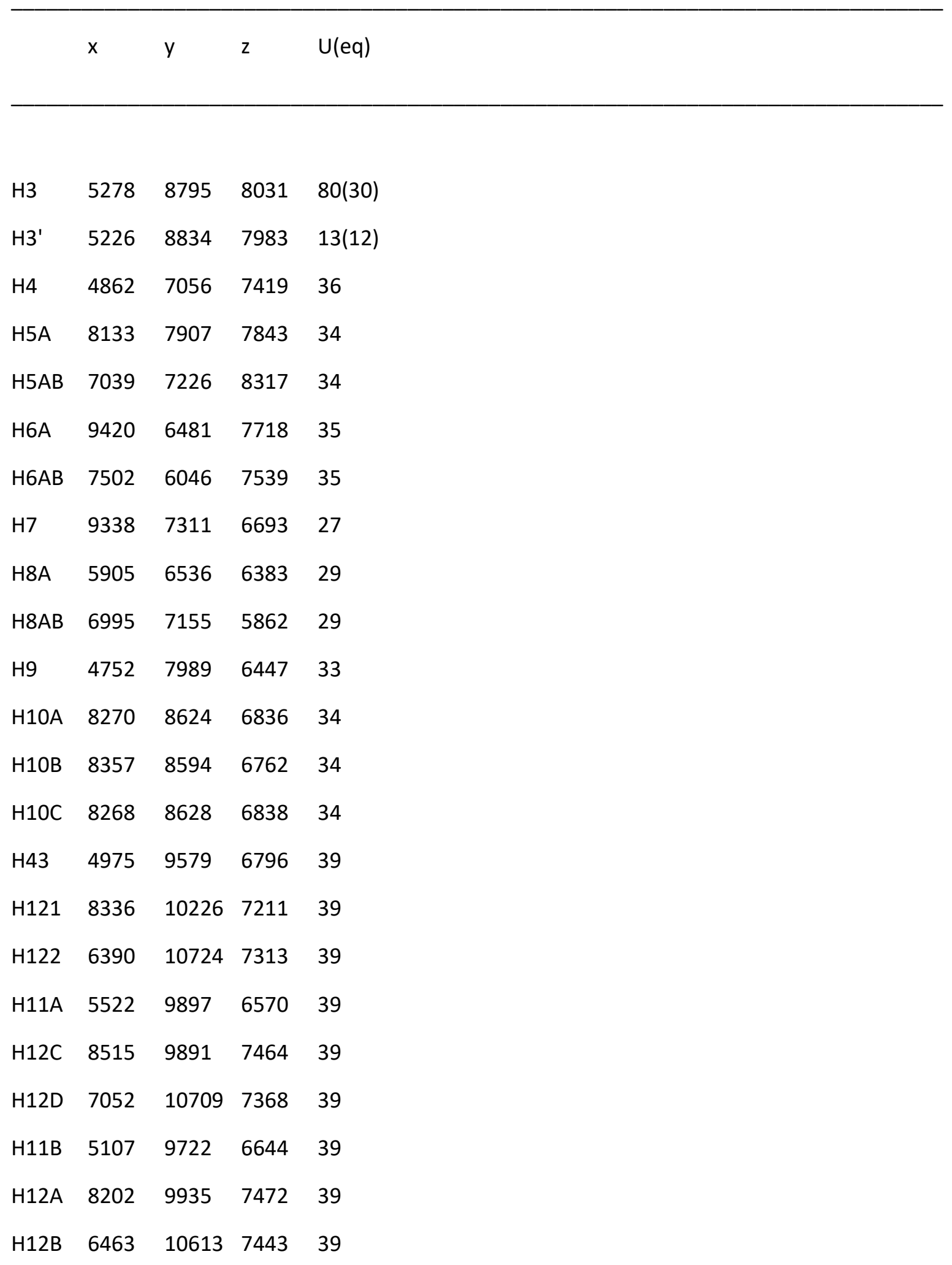




$\begin{array}{lllll}\text { H14A } & 5088 & 8951 & 5446 & 43 \\ \text { H15A } & 5823 & 9142 & 4270 & 44 \\ \text { H16A } & 8804 & 9127 & 3945 & 42 \\ \text { H17A } & 11066 & 8848 & 4772 & 40 \\ \text { H18A } & 10304 & 8691 & 5937 & 39 \\ \text { H14B } & 4656 & 8854 & 5514 & 47 \\ \text { H15B } & 5054 & 9059 & 4329 & 43 \\ \text { H16B } & 7948 & 9174 & 3890 & 42 \\ \text { H17B } & 10444 & 9083 & 4636 & 39 \\ \text { H18B } & 10046 & 8878 & 5821 & 43 \\ \text { H14C } & 5390 & 9115 & 5236 & 51 \\ \text { H15C } & 6610 & 9245 & 4196 & 46 \\ \text { H16C } & 9553 & 8974 & 3992 & 41 \\ \text { H17C } & 11551 & 8636 & 4907 & 38 \\ \text { H18C } & 10421 & 8543 & 6031 & 31 \\ \text { H21 } & 9048 & 3434 & 5657 & 32 \\ \text { H22 } & 11761 & 2861 & 5240 & 35 \\ \text { H23 } & 14334 & 3737 & 5237 & 37 \\ \text { H24 } & 14299 & 5216 & 5665 & 35\end{array}$




\section{Stereochemical assignment of amide $4 \mathbf{j}$}

NMR spectroscopy was employed to determine the diastereomer of $4 \mathbf{j}$. COSY, HSQC, and HMBC, were used to assign proton and carbon signals in $\mathrm{CDCl}_{3}$. NOESY 1D with selective inversion of key peaks were used to assign stereochemistry at $\mathrm{C} 6$. nOe interactions were found between $\mathrm{H} 5$ and $\mathrm{H} 26$ in $\mathrm{CDCl}_{3}$. The lack of between $\mathrm{H} 6$ with $\mathrm{H} 2 \mathrm{ax}$ and $\mathrm{H} 4 \mathrm{ax}$ in $\mathrm{C}_{6} \mathrm{D}_{6}$ supports $\mathrm{H} 6$ being equatorial.

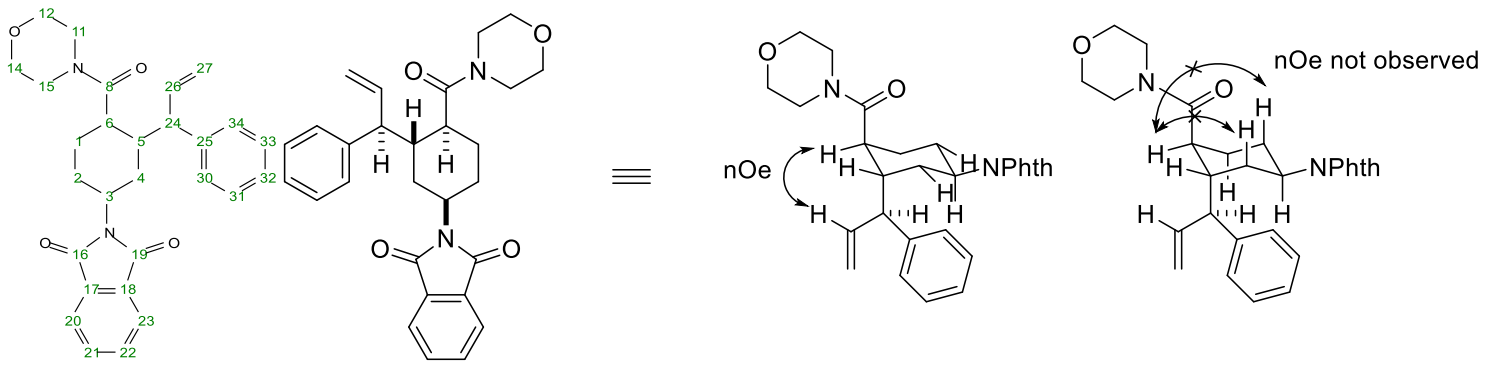

$\left(600 \mathrm{MHz}, \mathrm{CDCl}_{3}\right)$

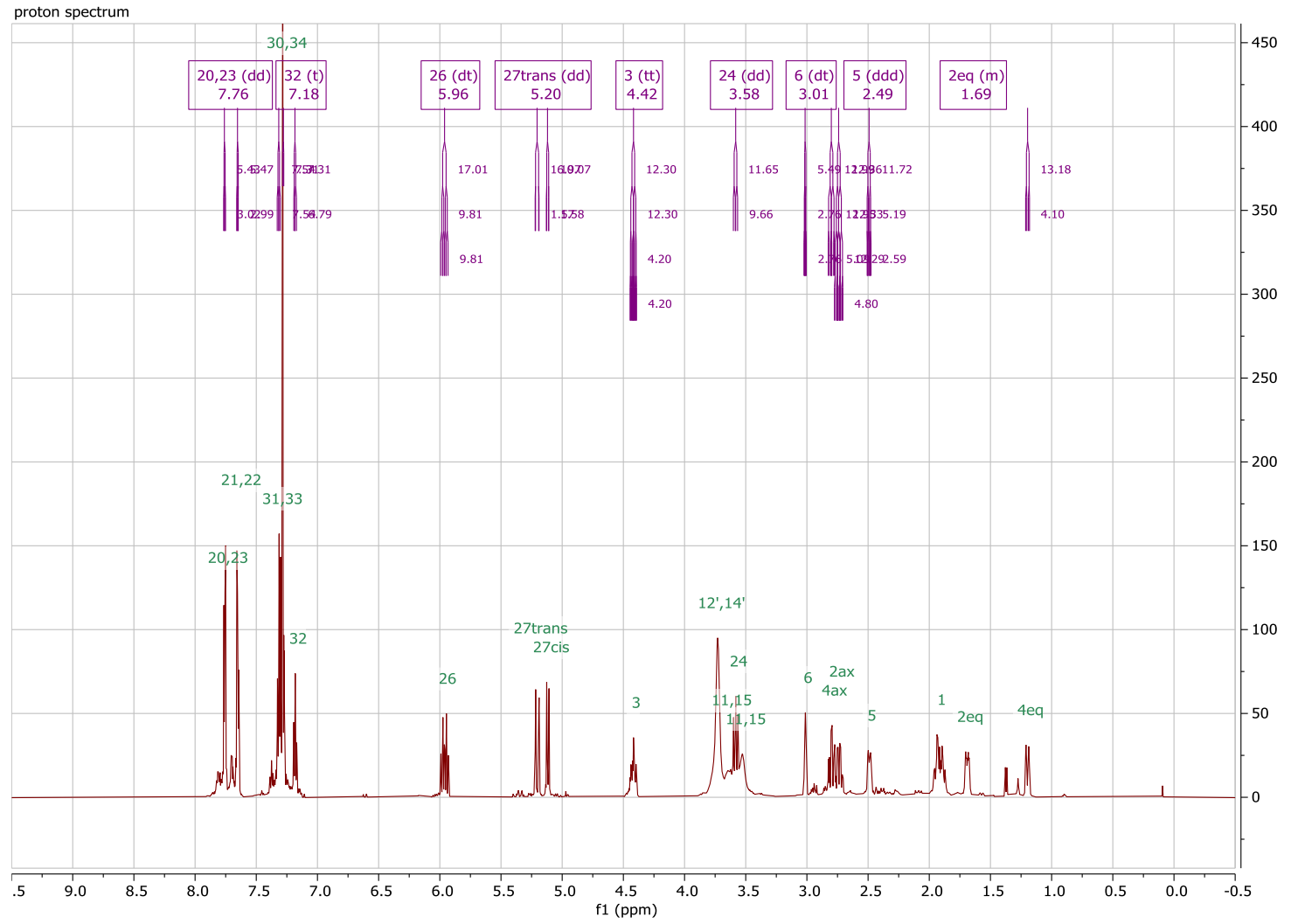


(151 MHz, $\mathrm{CDCl}_{3}$ )
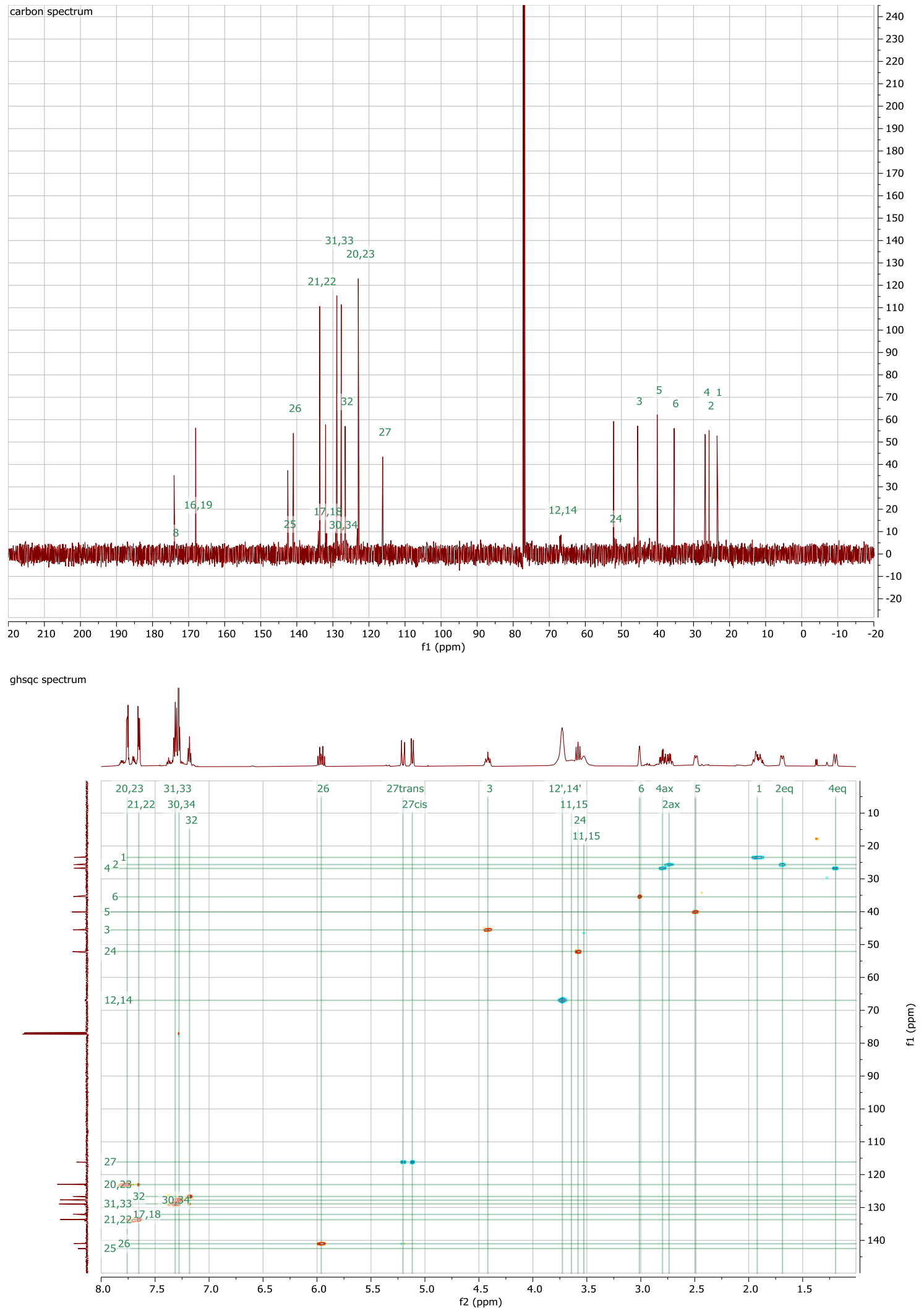


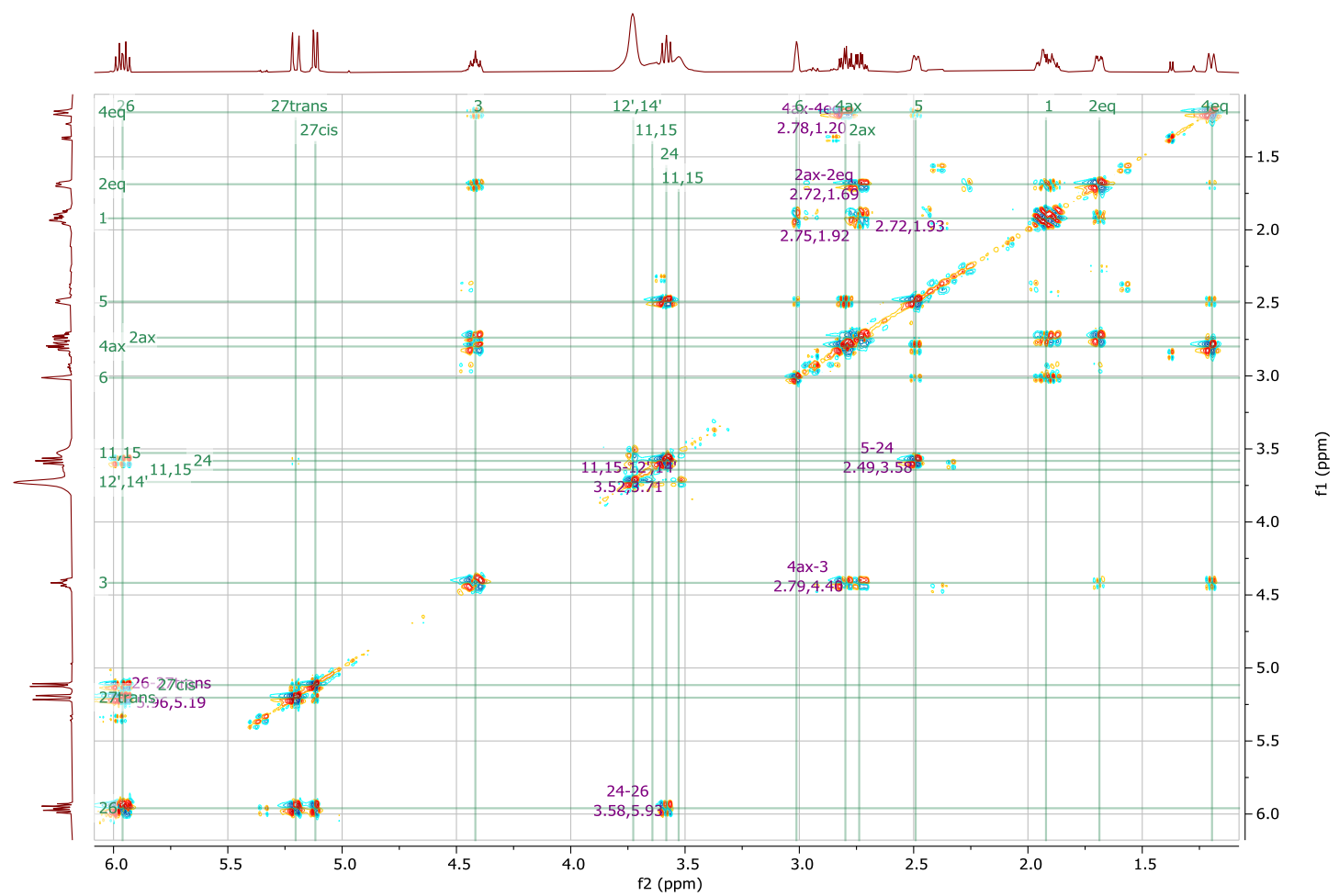

ghmbc spectrum

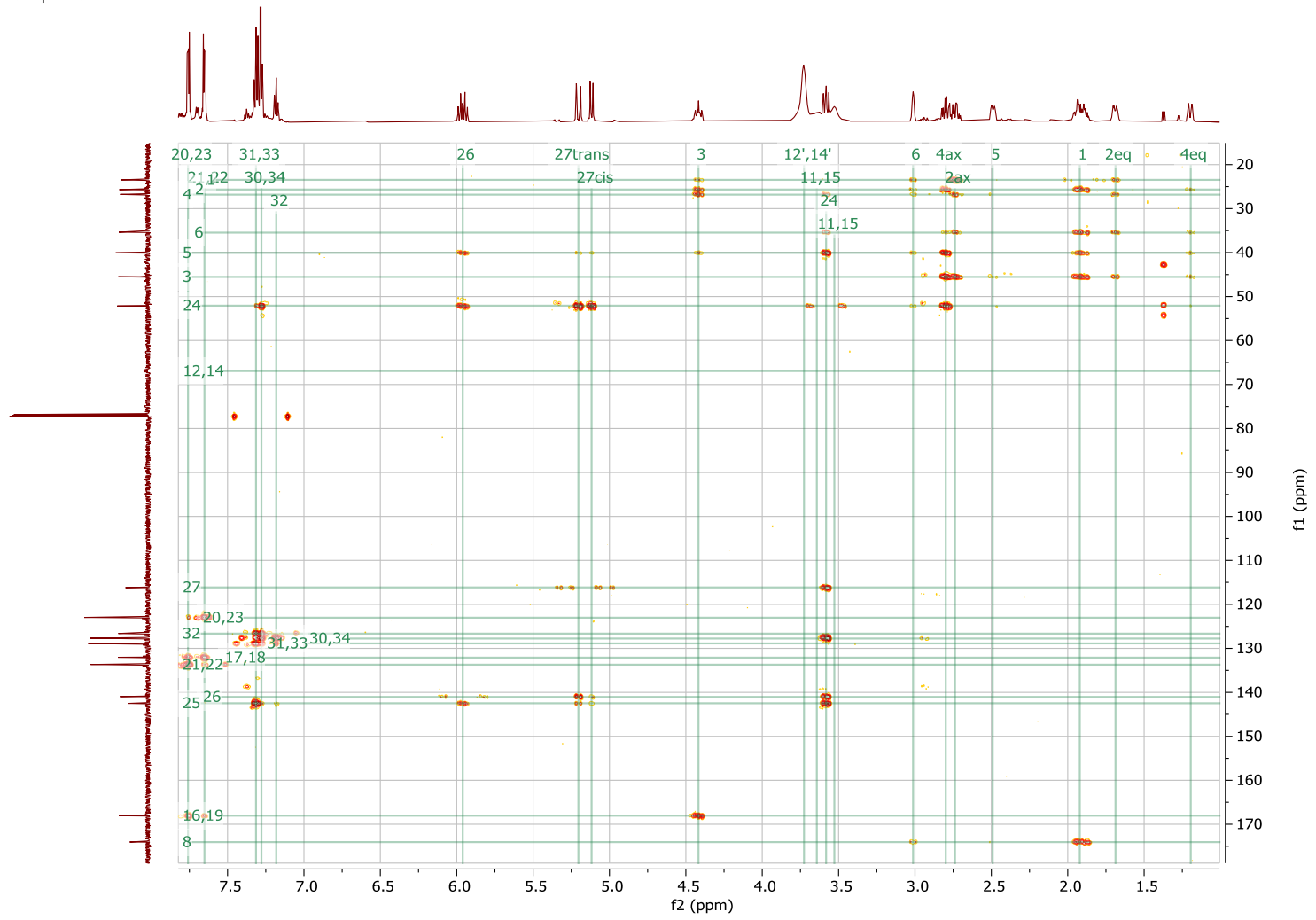




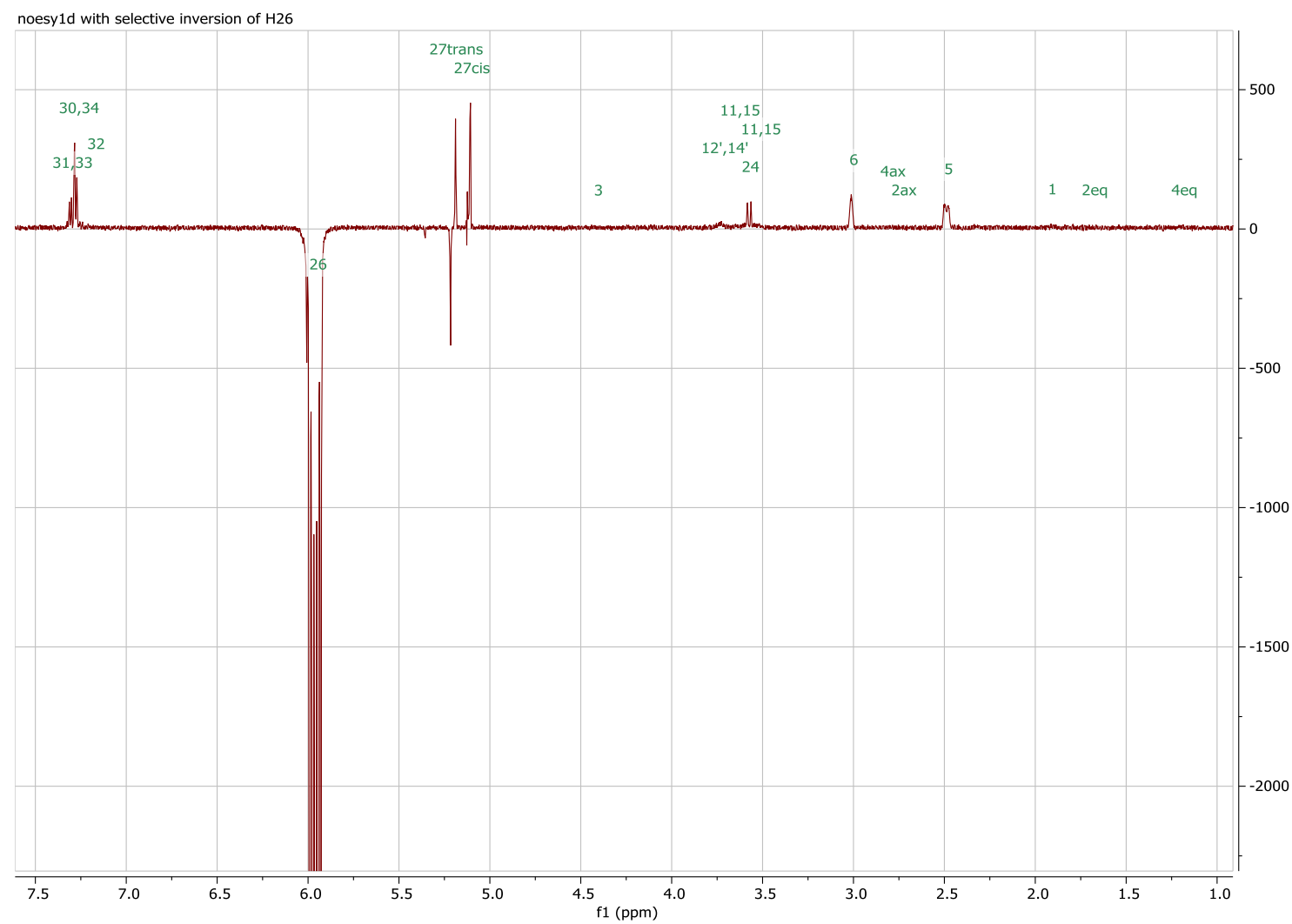

(600 MHz, $\mathrm{C}_{6} \mathrm{D}_{6}$ )

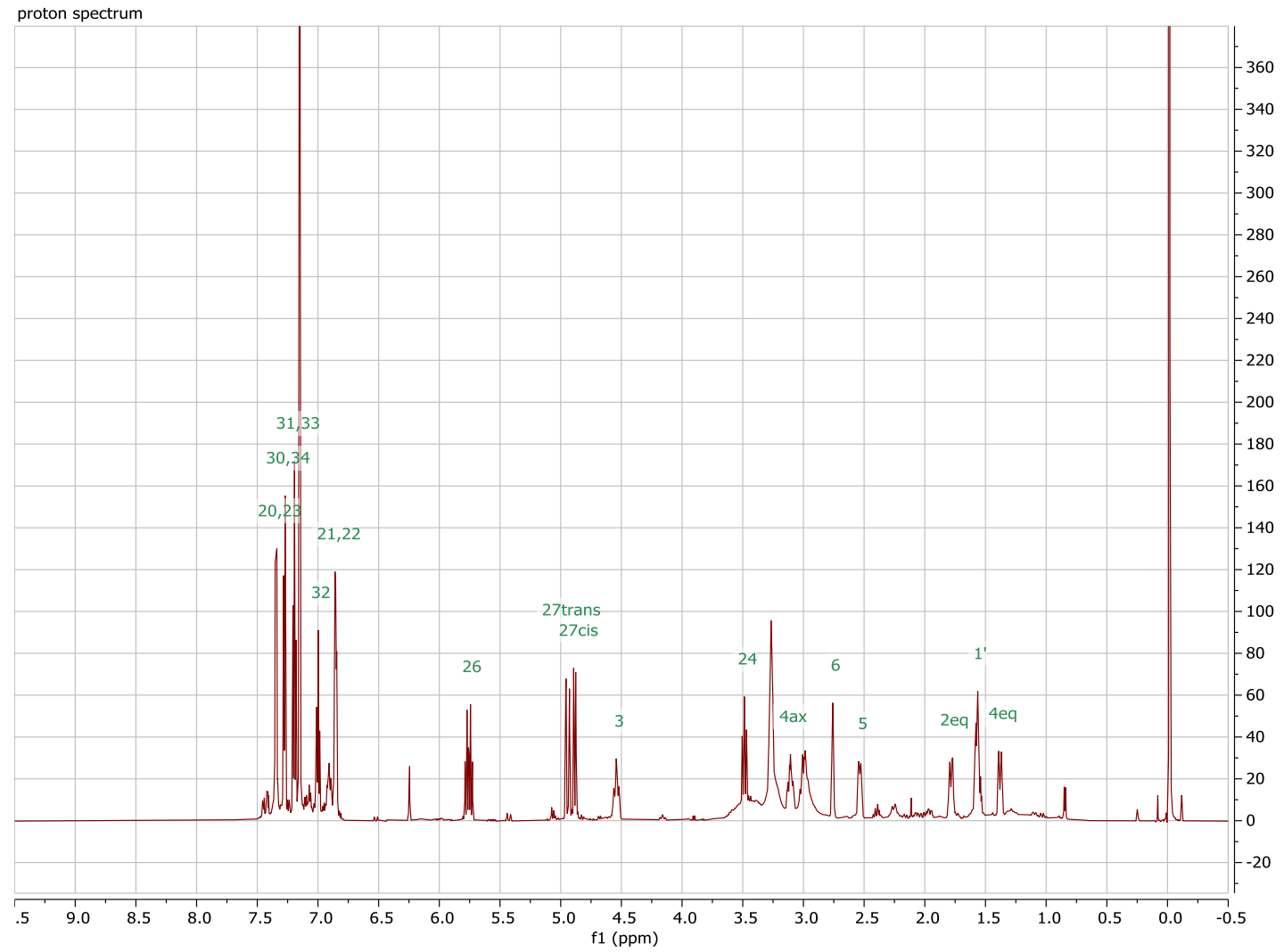




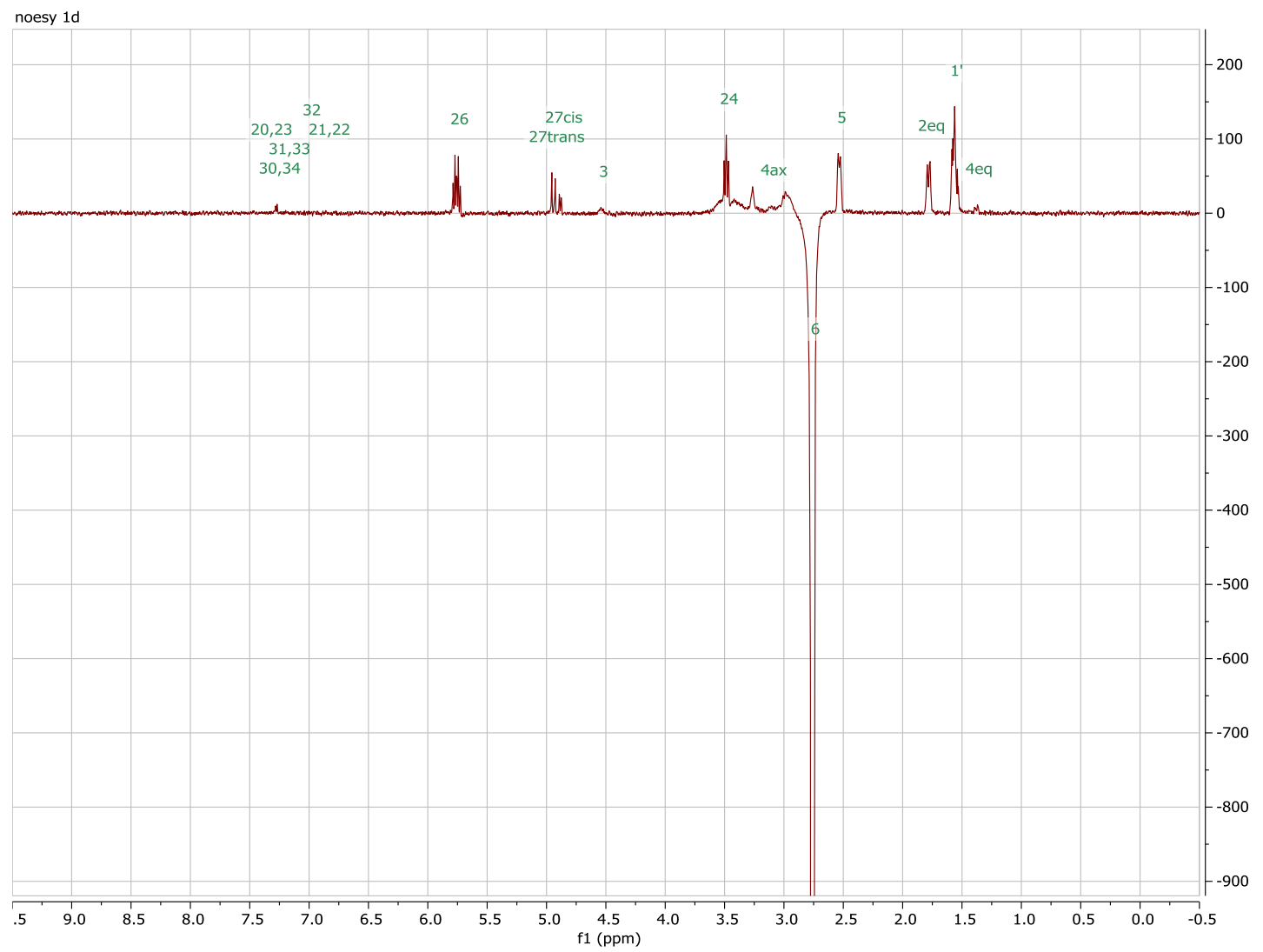




\section{References}

(1) Abdel-Mohsen, H. T.; Conrad, J.; Beifuss, U. Green Chem. 2012, 14 (10), 2686-2690. https://doi.org/10.1039/c2gc35950b.

(2) Burmaoglu, S.; Yilmaz, A. O.; Taslimi, P.; Algul, O.; Kilic, D.; Gulcin, I. Arch. Pharm. (Weinheim). 2018, 351 (2), 1-12. https://doi.org/10.1002/ardp.201700314.

(3) West, T. H.; Daniels, D. S. B.; Slawin, A. M. Z.; Smith, A. D. J. Am. Chem. Soc. 2014, 136 (12), 44764479. https://doi.org/10.1021/ja500758n.

(4) Zhang, Y.; Xiong, W.; Cen, J.; Yan, W.; Wu, Y.; Qi, C.; Wu, W.; Jiang, H. Chem. Commun. 2019, 55 (82), 12304-12307. https://doi.org/10.1039/c9cc05495b.

(5) Chen, D. D.; Zhang, B. Y.; Liu, X. X.; Li, X. Q.; Yang, X. J.; Zhou, L. Bioorganic Med. Chem. Lett. 2018, 28 (6), 1149-1153. https://doi.org/10.1016/j.bmcl.2017.08.051.

(6) Štambaský, J.; Malkov, A. V.; Kočovsky, P. Collect. Czechoslov. Chem. Commun. 2008, 73 (5), 705732. https://doi.org/10.1135/cccc20080705.

(7) Liu, J.; Cao, C. G.; Sun, H. B.; Zhang, X.; Niu, D. J. Am. Chem. Soc. 2016, 138 (40), 13103-13106. https://doi.org/10.1021/jacs.6b05288.

(8) Scott, S. K.; Grenning, A. J. Angew. Chemie - Int. Ed. 2017, 56 (28), 8125-8129. https://doi.org/10.1002/anie.201703186.

(9) Vertesaljai, P.; Ghiviriga, I.; Grenning, A. J. Org. Lett. 2018, 20 (7), 1970-1973. https://doi.org/10.1021/acs.orglett.8b00499.

(10) Hayashi, Y.; Li, J.; Asano, H.; Sakamoto, D. European J. Org. Chem. 2019, 2019 (4), 675-677. https://doi.org/10.1002/ejoc.201800984.

(11) Zhang, L.; Clark, R. J.; Zhu, L. Chem. - A Eur. J. 2008, 14 (9), 2894-2903. https://doi.org/10.1002/chem.200701419.

(12) Emmetiere, F.; Grenning, A. J. Synth. 2020, 52 (2), 3077-3085. https://doi.org/10.1055/s-00401707184.

(13) Chung, M. C.; Chan, Y. H.; Chang, W. J.; Hou, D. R. Org. Biomol. Chem. 2017, 15 (17), 3783-3790. https://doi.org/10.1039/c7ob00528h.

(14) Sheldrick, G. M. Acta Crystallogr. Sect. C Struct. Chem. 2015, 71 (Md), 3-8. https://doi.org/10.1107/S2053229614024218. 


\section{NMR spectral reprints}

2-(4-(1,3-dioxoisoindolin-2-yl)cyclohexylidene)malononitrile (SI-1) ${ }^{1} \mathrm{H}$ Spectrum $\left(600 \mathrm{MHz}, \mathrm{CDCl}_{3}\right)$

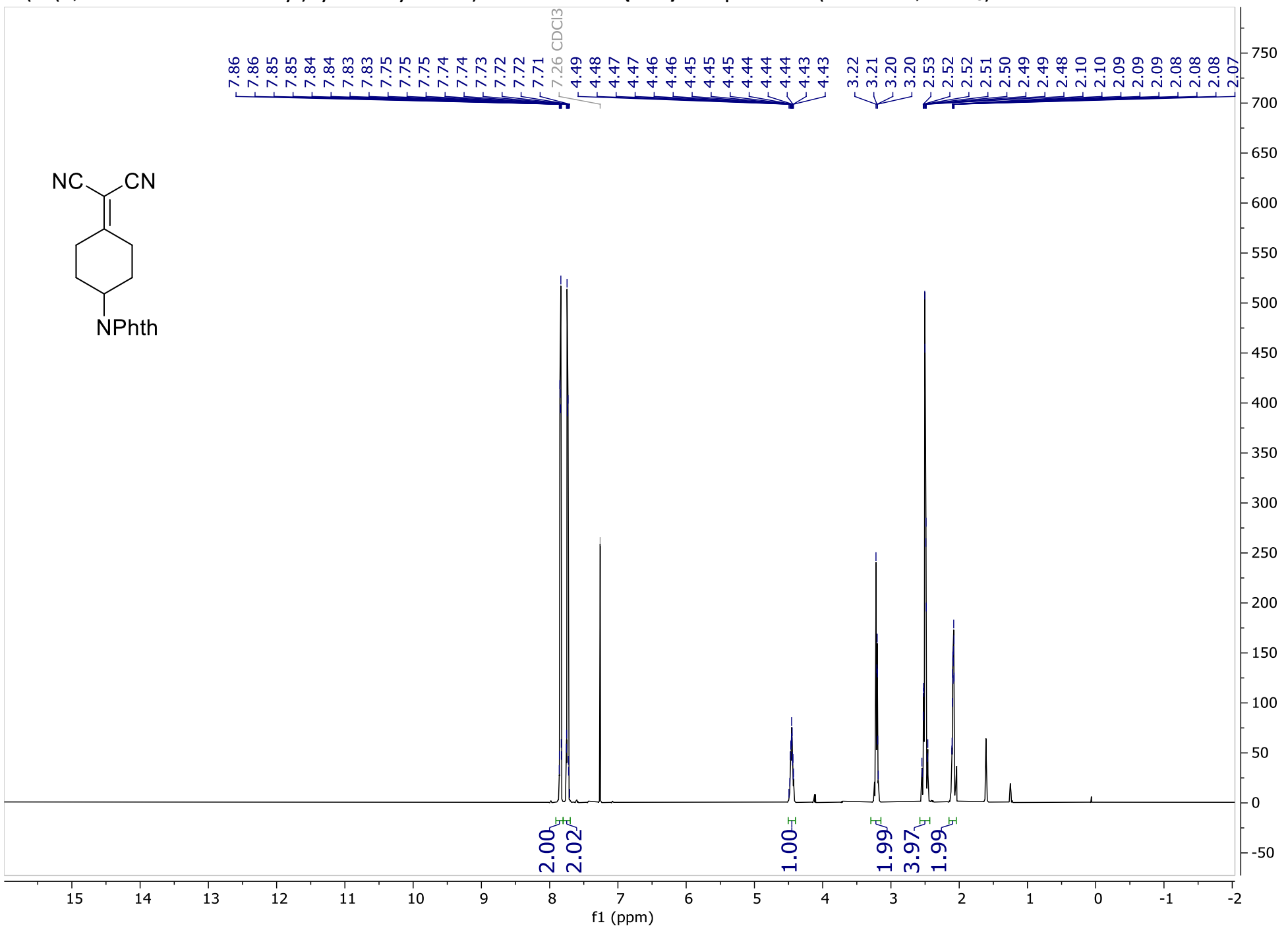


2-(4-(1,3-dioxoisoindolin-2-yl)cyclohexylidene)malononitrile (SI-1) (151 MHz, $\mathrm{CDCl}_{3}$ )
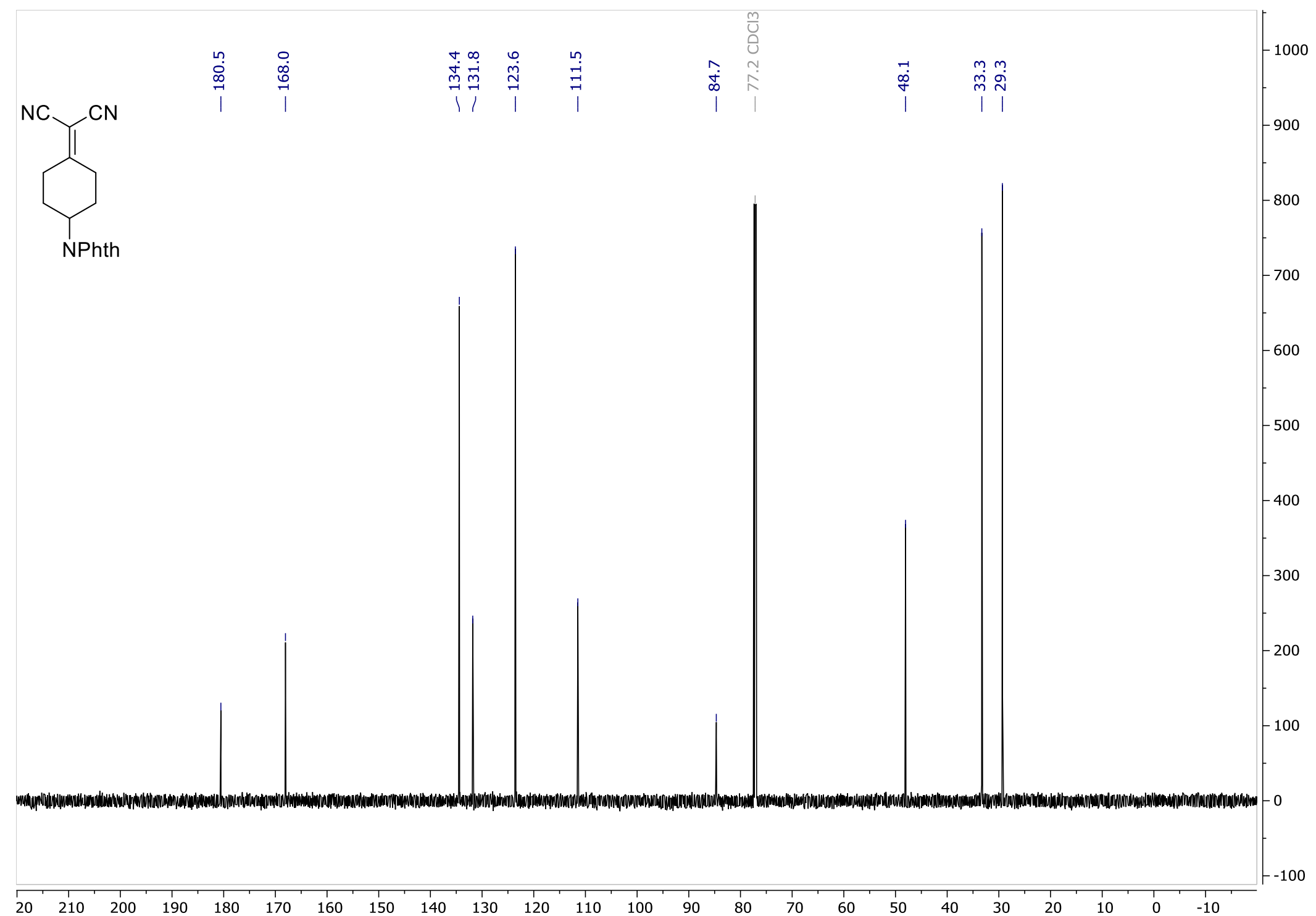

f1 (ppm) 


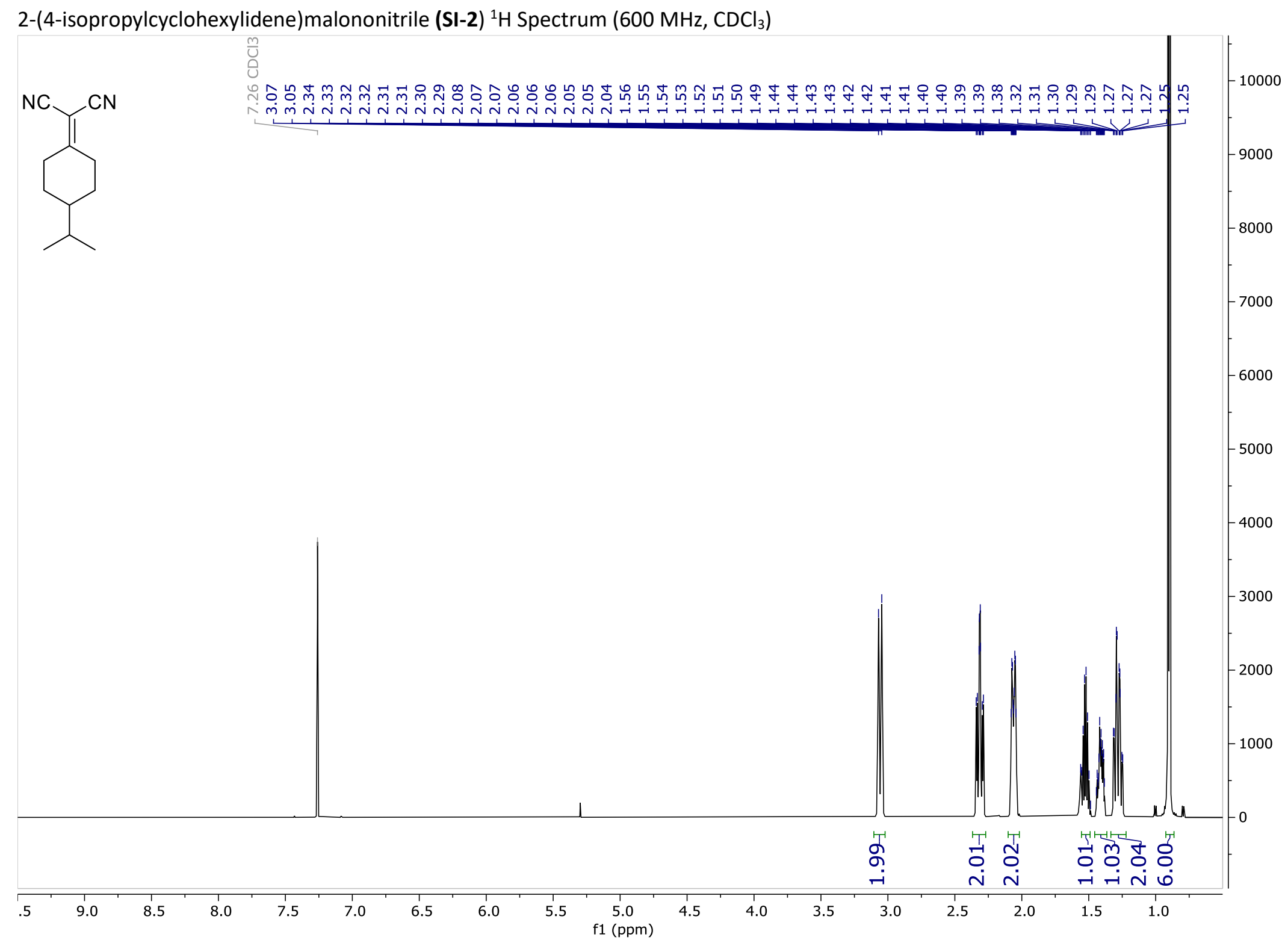


2-(4-isopropylcyclohexylidene)malononitrile (SI-2) ${ }^{13} \mathrm{C}$ Spectrum (151 MHz, $\mathrm{CDCl}_{3}$ )

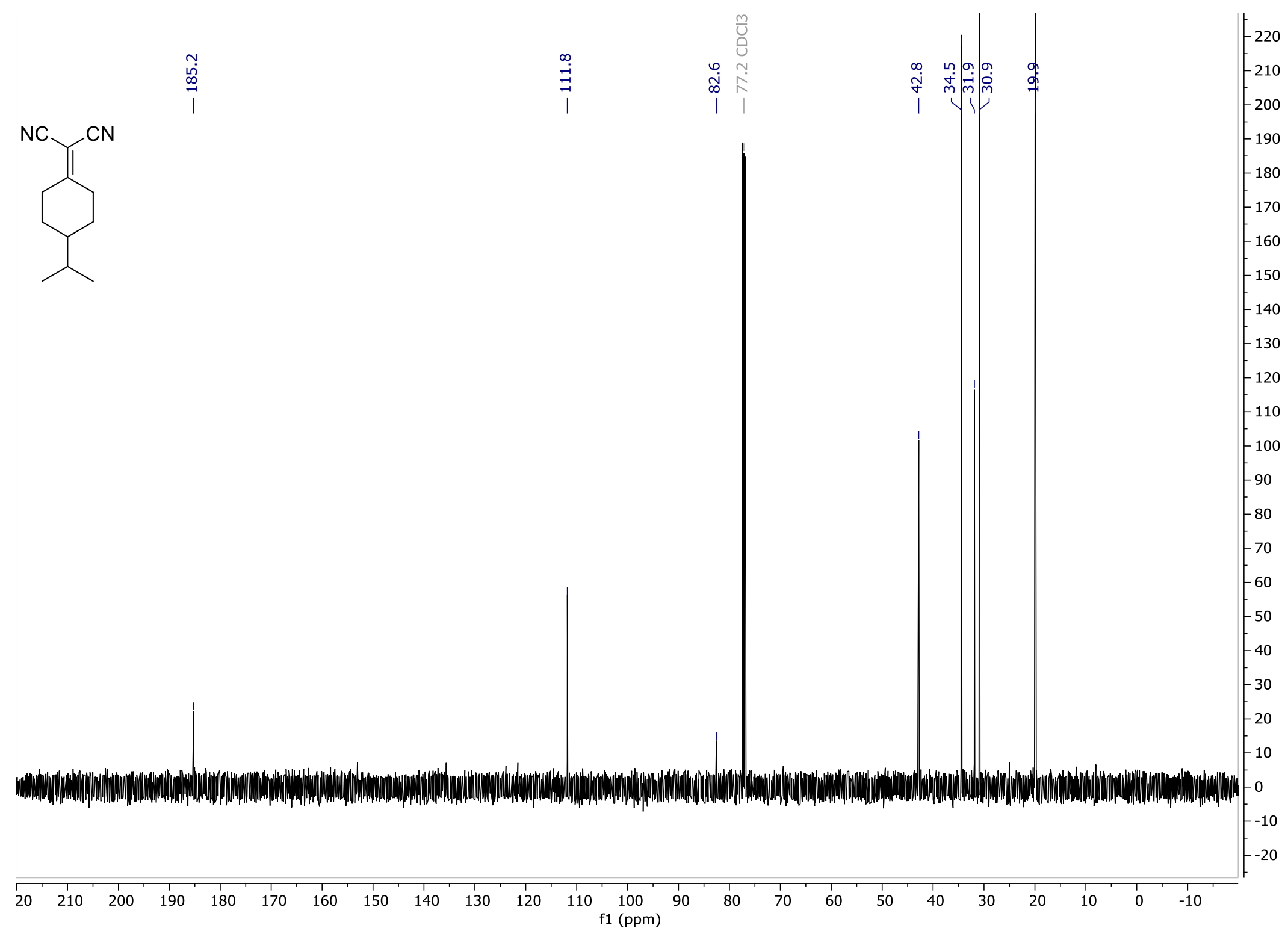




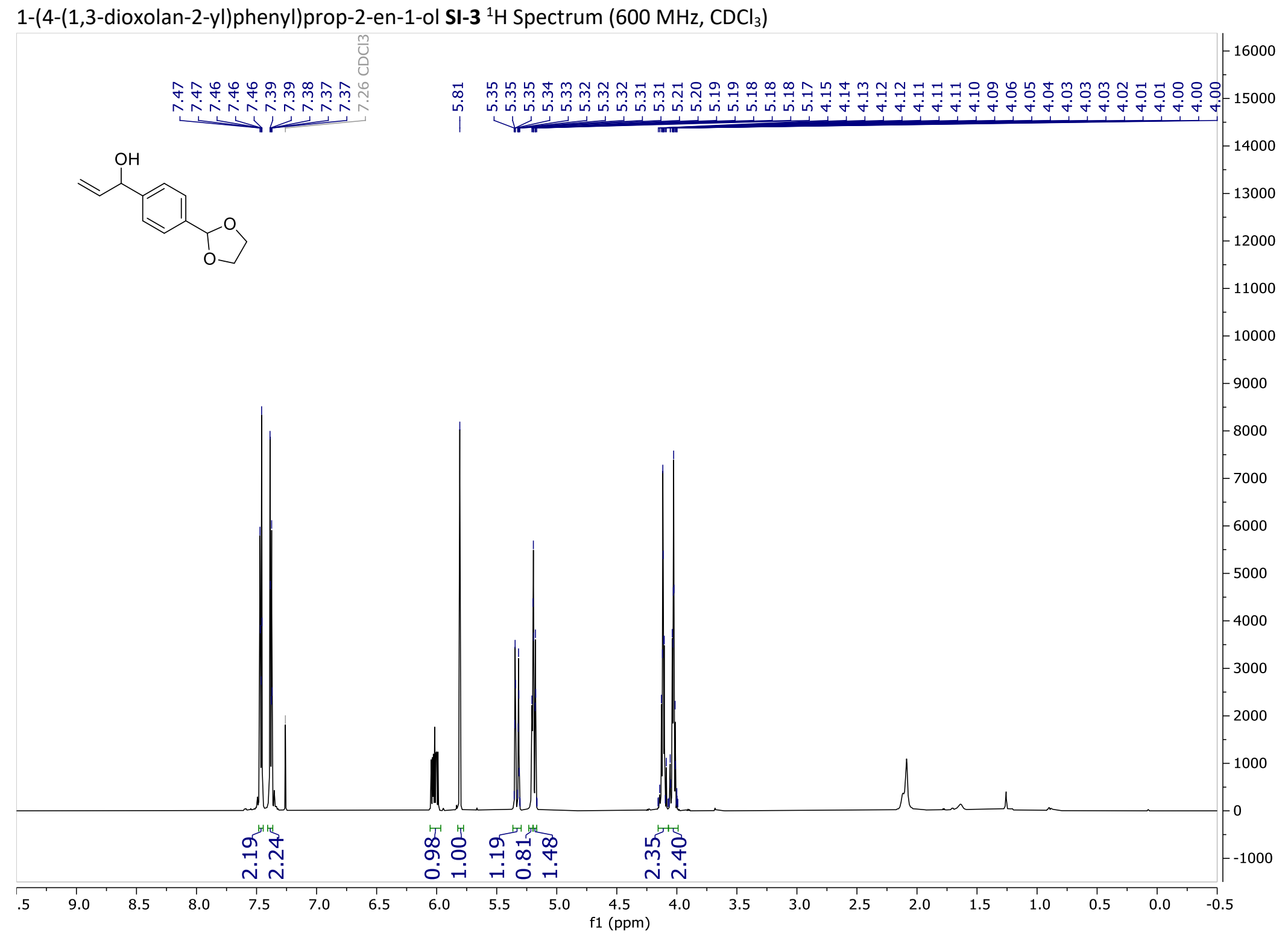


1-(4-(1,3-dioxolan-2-yl)phenyl)prop-2-en-1-ol SI-3 ${ }^{13} \mathrm{C}$ Spectrum (151 MHz, CDCl 3 )

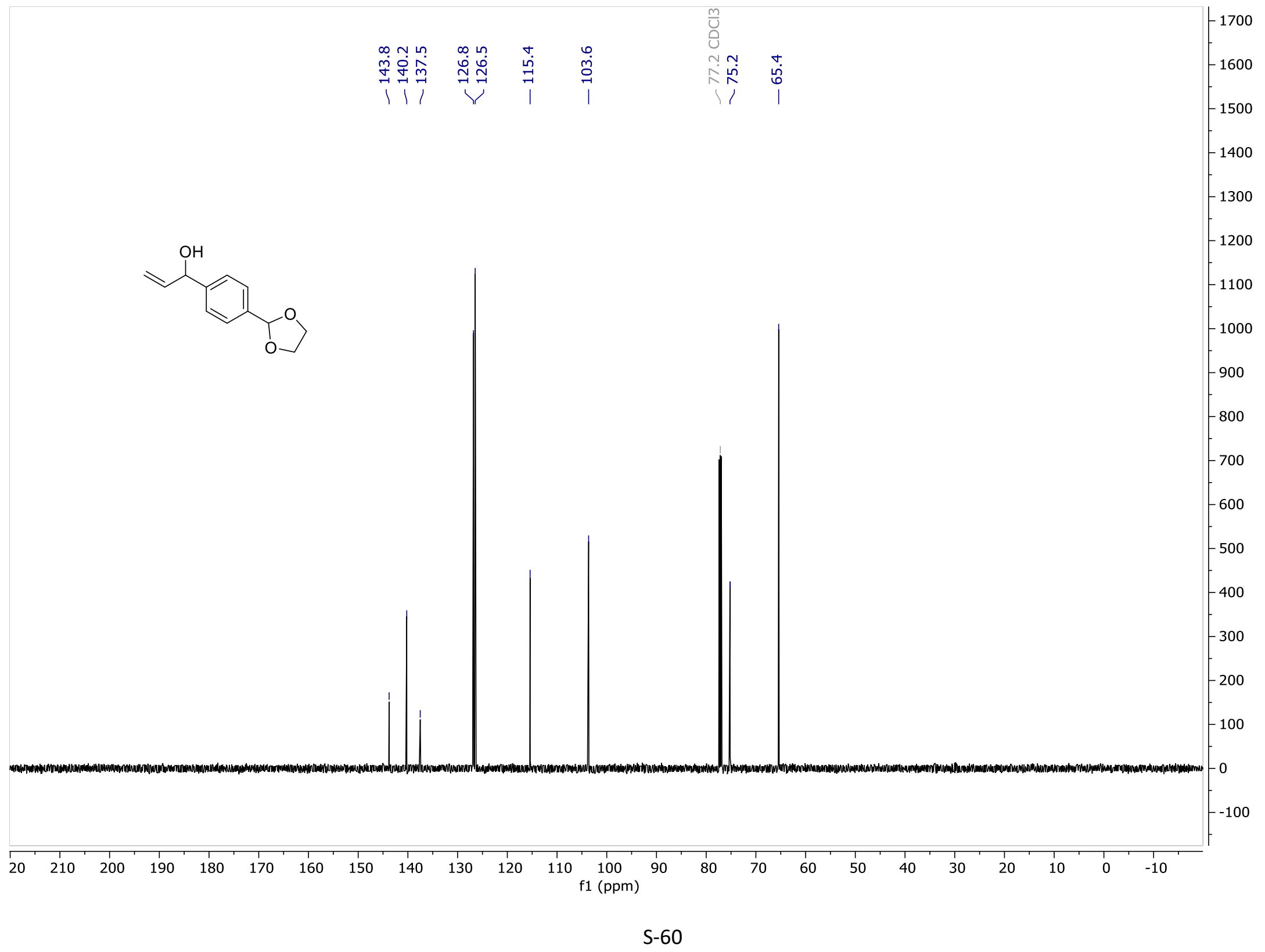


1-(4-(1,3-dioxolan-2-yl)phenyl)allyl tert-butyl carbonate SI-s4 ${ }^{1} \mathrm{H}$ Spectrum $\left(600 \mathrm{MHz}, \mathrm{CDCl}_{3}\right)$

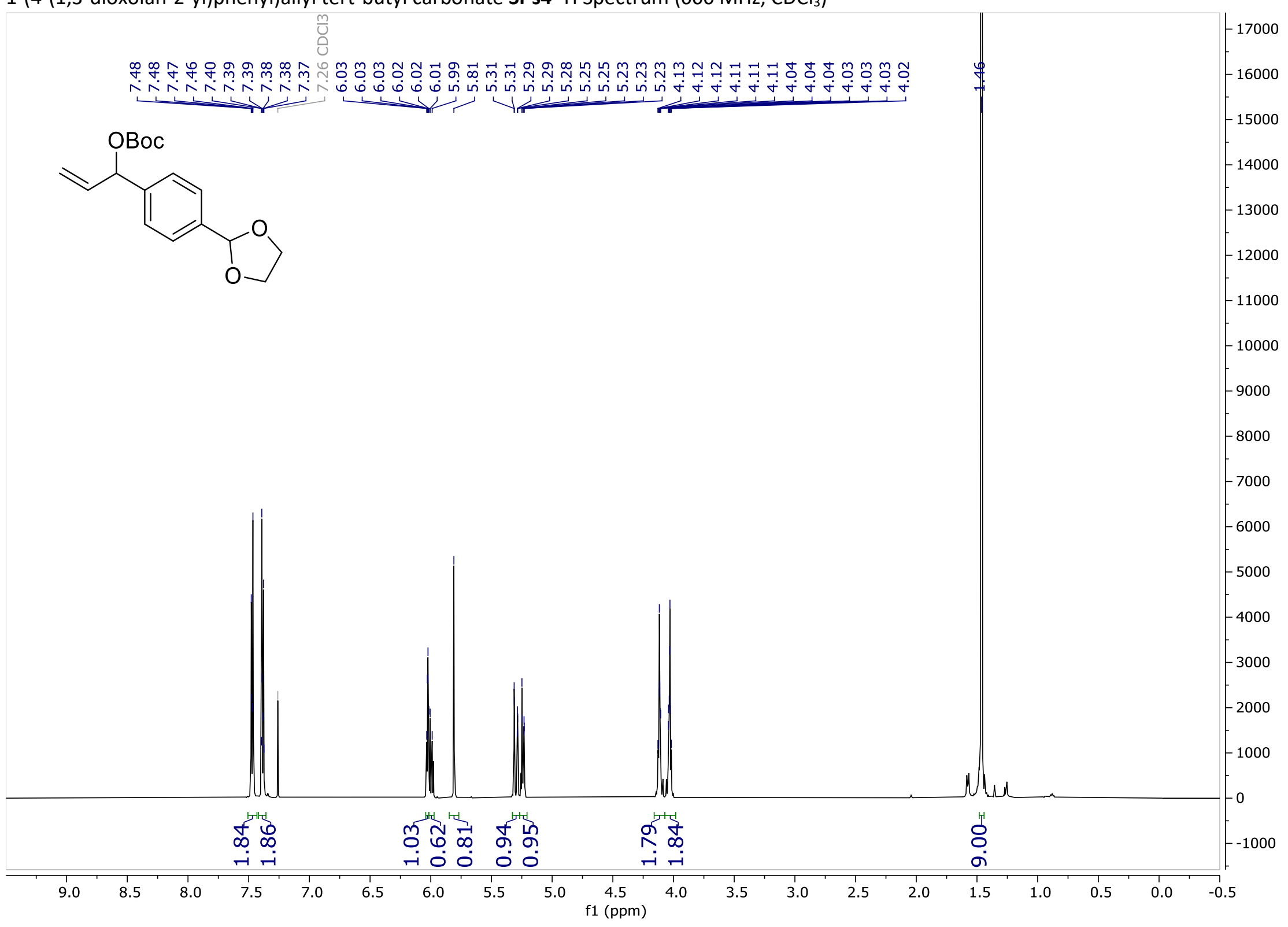


1-(4-(1,3-dioxolan-2-yl)phenyl)allyl tert-butyl carbonate SI-4 ${ }^{13} \mathrm{C}$ Spectrum (151 MHz, CDCl 3 )

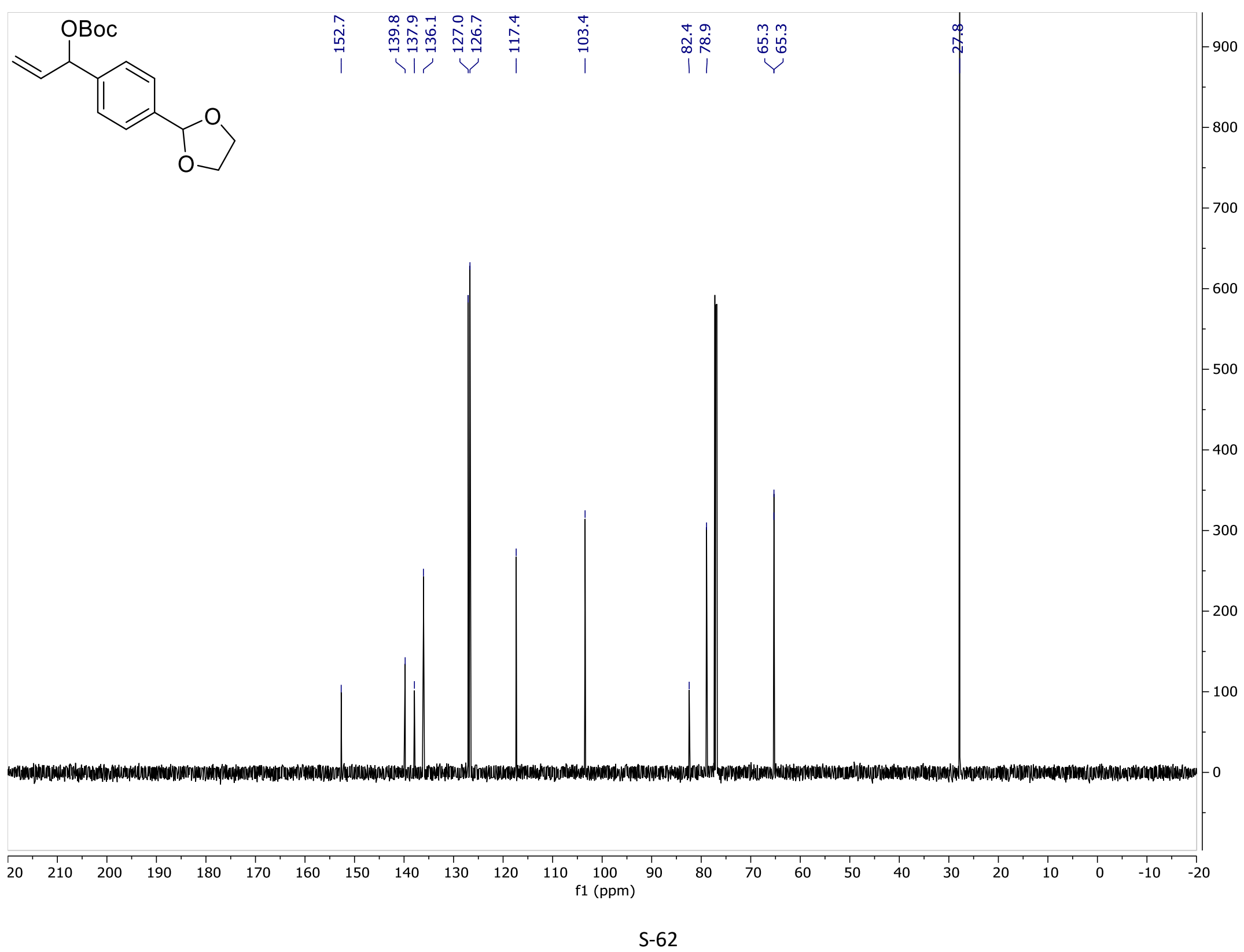


2-cinnamyl-2-(1,2,3,6-tetrahydro-[1,1'-biphenyl]-4-yl)malononitrile (1a) ${ }^{1} \mathrm{H}$ Spectrum $\left(600 \mathrm{MHz}, \mathrm{CDCl}_{3}\right)$

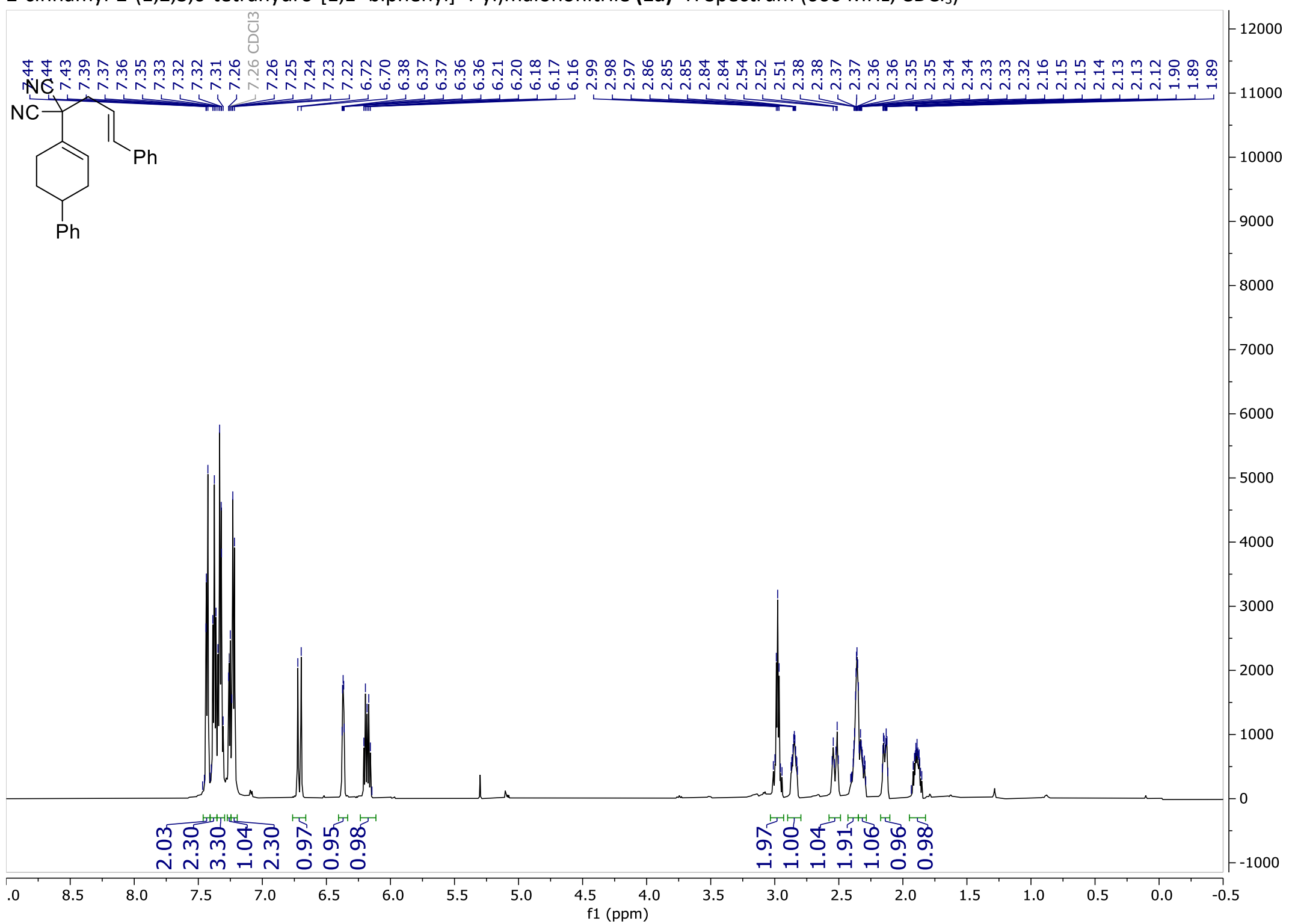


2-cinnamyl-2-(1,2,3,6-tetrahydro-[1,1'-biphenyl]-4-yl)malononitrile (1a) ${ }^{13} \mathrm{C}$ Spectrum $\left(151 \mathrm{MHz}, \mathrm{CDCl}_{3}\right)$

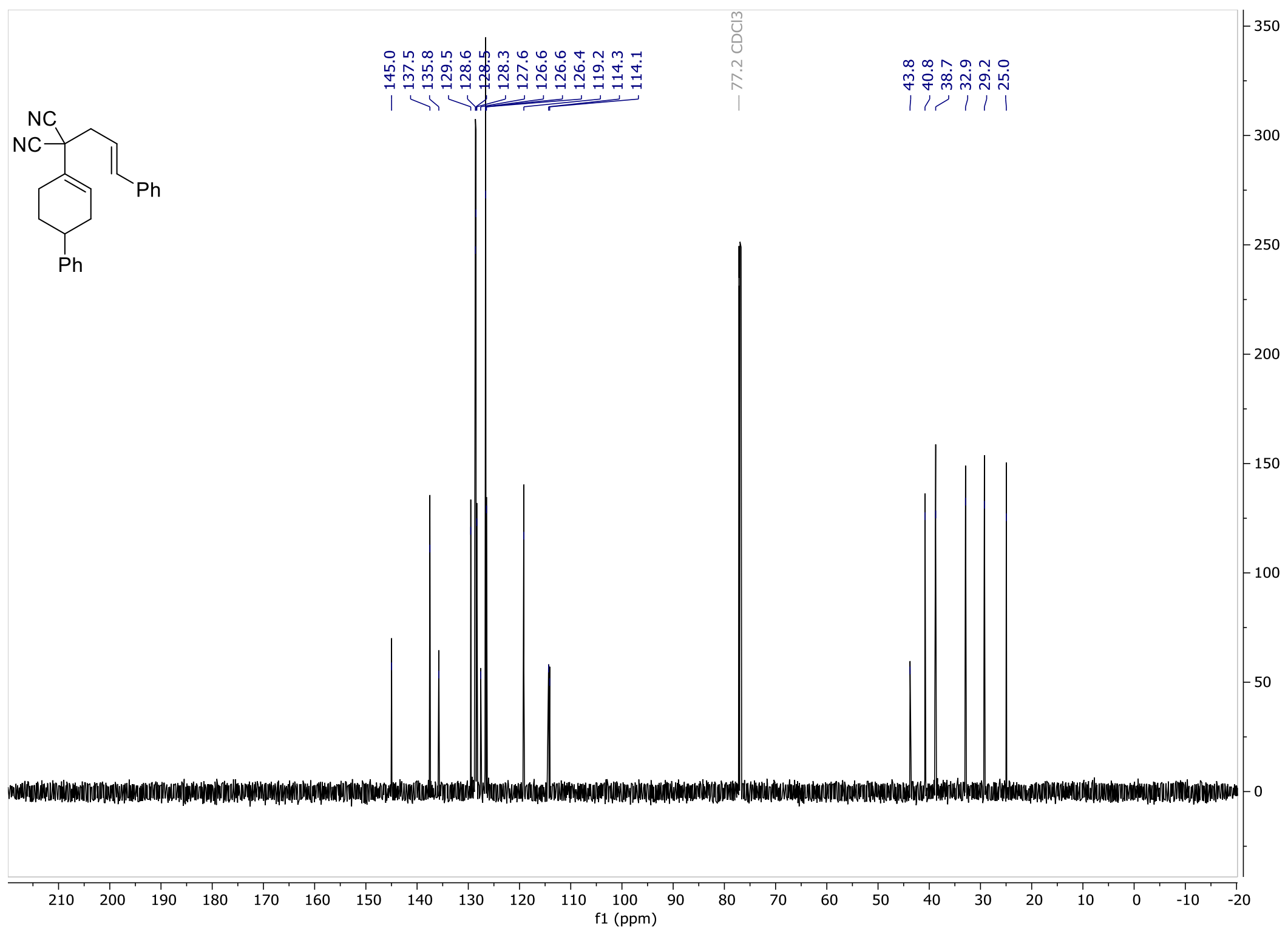


2-cinnamyl-2-(4-methylcyclohex-1-en-1-yl)malononitrile (1b) ${ }^{1} \mathrm{H}$ Spectrum $\left(600 \mathrm{MHz}, \mathrm{CDCl}_{3}\right.$ )

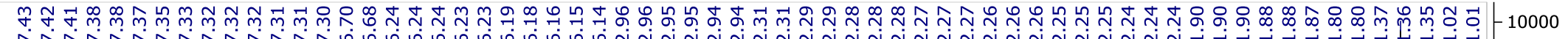
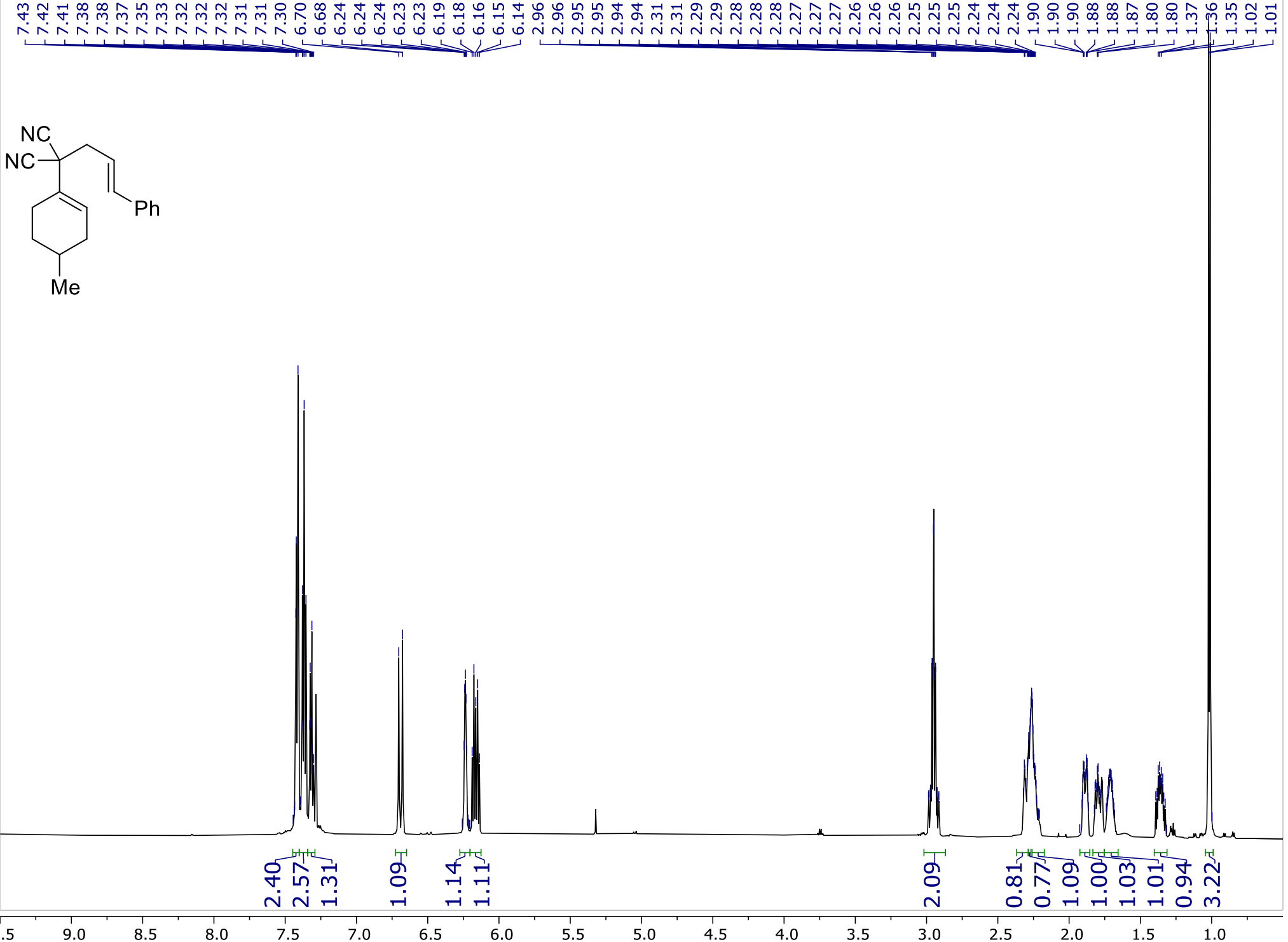
2-cinnamyl-2-(4-methylcyclohex-1-en-1-yl)malononitrile (1) ${ }^{13} \mathrm{C}$ Spectrum $\left(151 \mathrm{MHz}, \mathrm{CDCl}_{3}\right)$

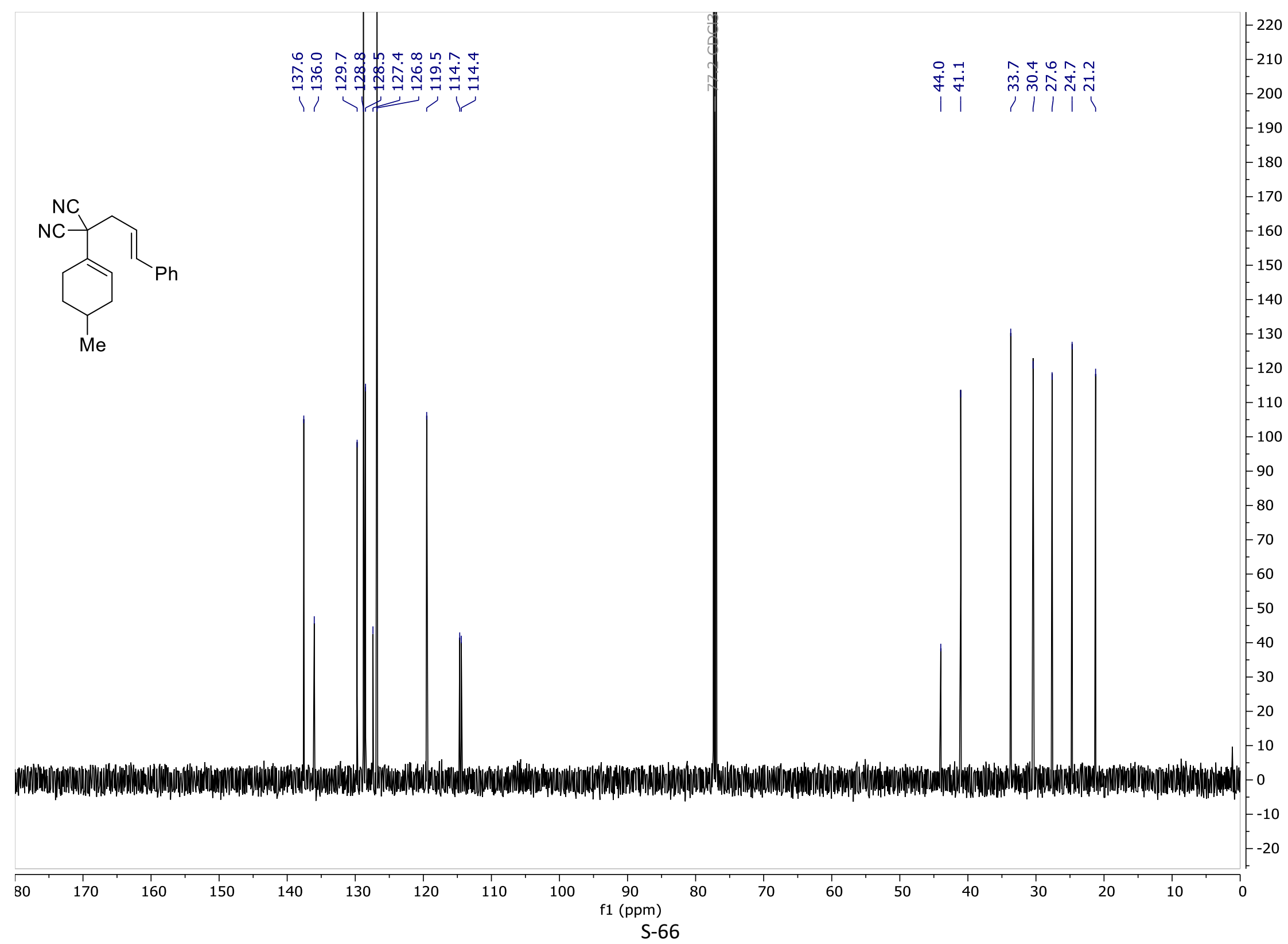


2-cinnamyl-2-(4-isopropylcyclohex-1-en-1-yl)malononitrile (1c) ${ }^{1} \mathrm{H}$ Spectrum $\left(600 \mathrm{MHz}, \mathrm{CDCl}_{3}\right)$

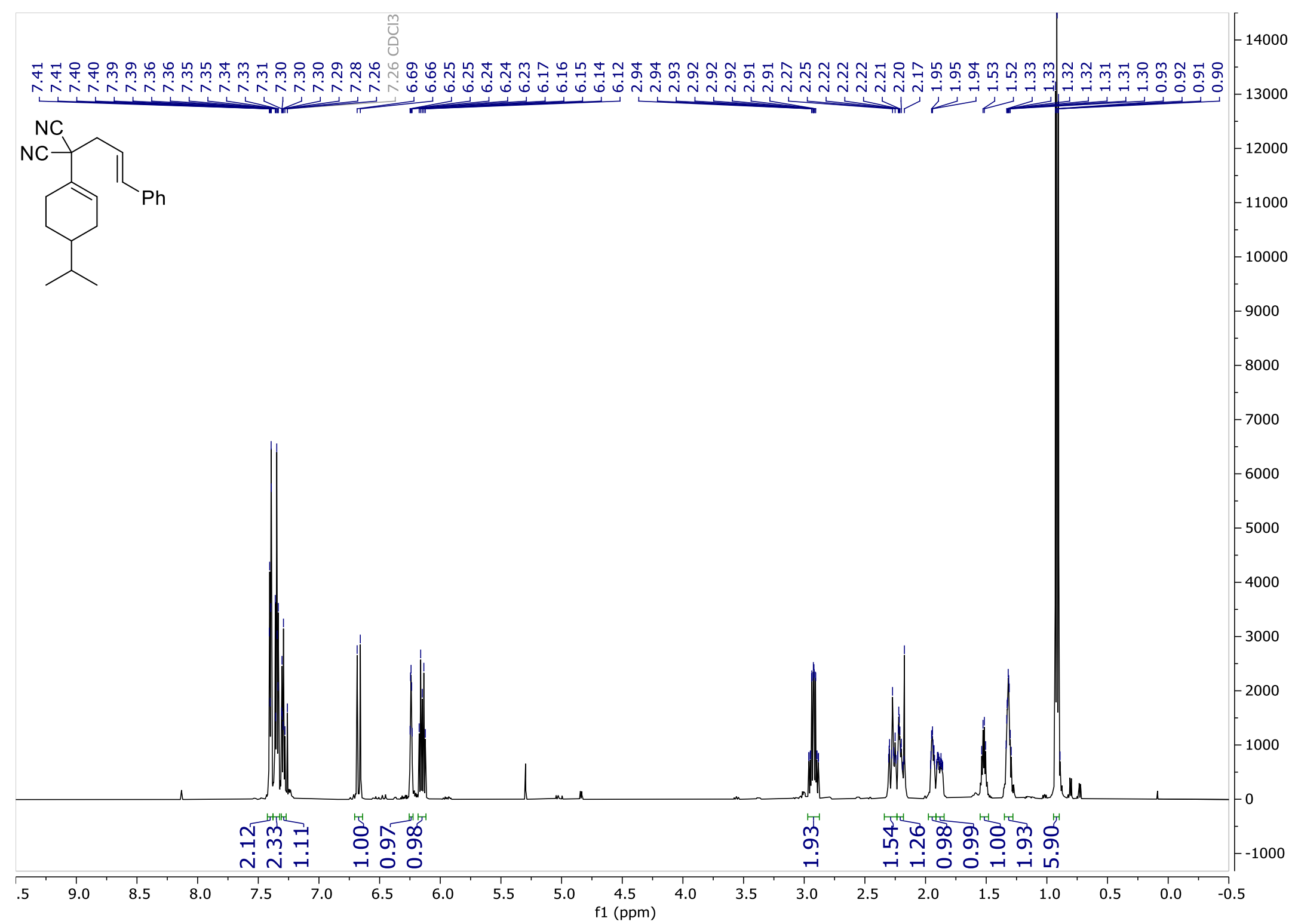


2-cinnamyl-2-(4-isopropylcyclohex-1-en-1-yl)malononitrile (1c) ${ }^{13} \mathrm{C}$ Spectrum $\left(151 \mathrm{MHz}, \mathrm{CDCl}_{3}\right.$ )

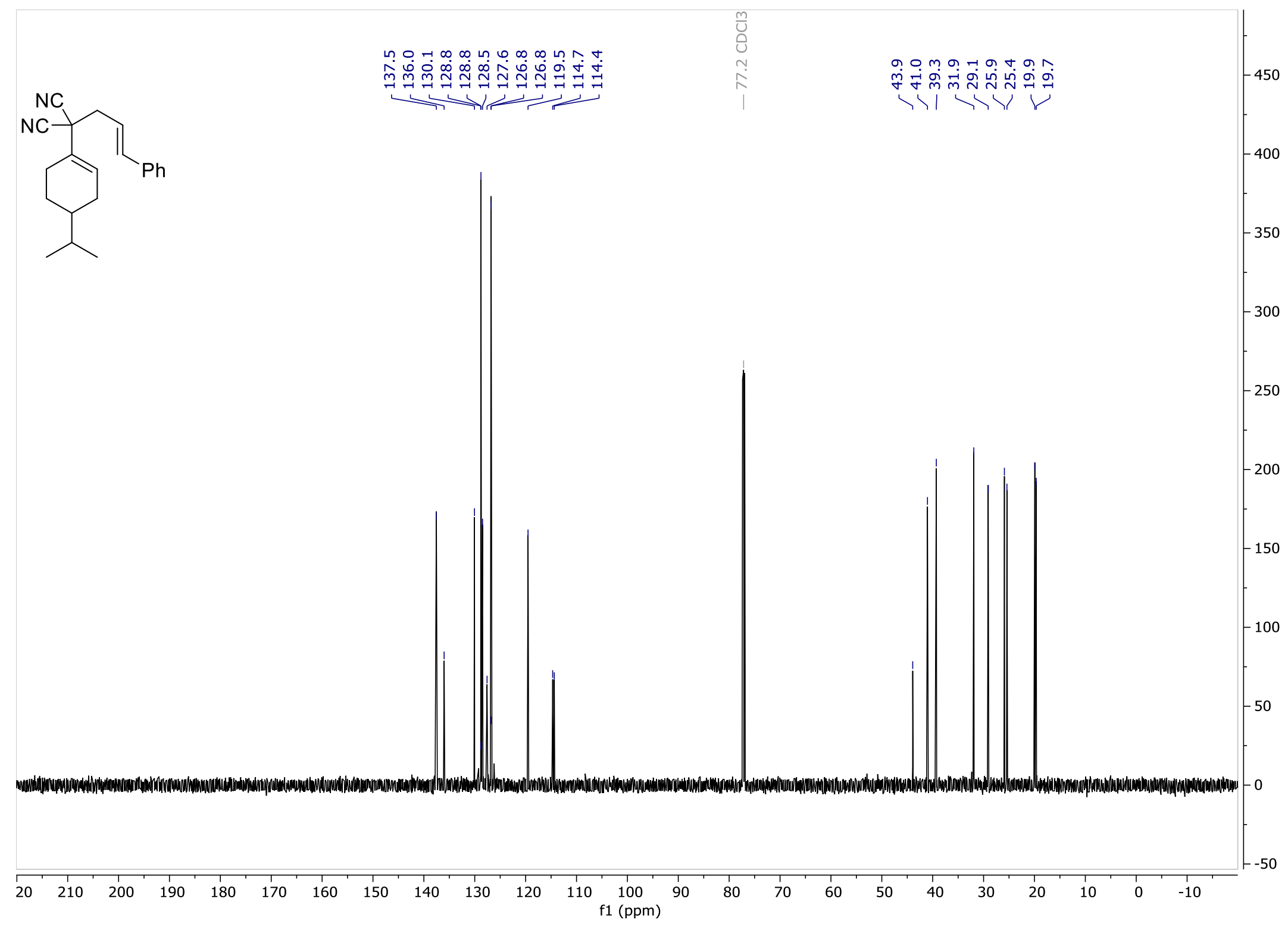


Ethyl (E)-4-(1,1-dicyano-4-phenylbut-3-en-1-yl)cyclohex-3-ene-1-carboxylate (1d) ${ }^{1} \mathrm{H}$ Spectrum $\left(600 \mathrm{MHz}, \mathrm{CDCl}_{3}\right)$

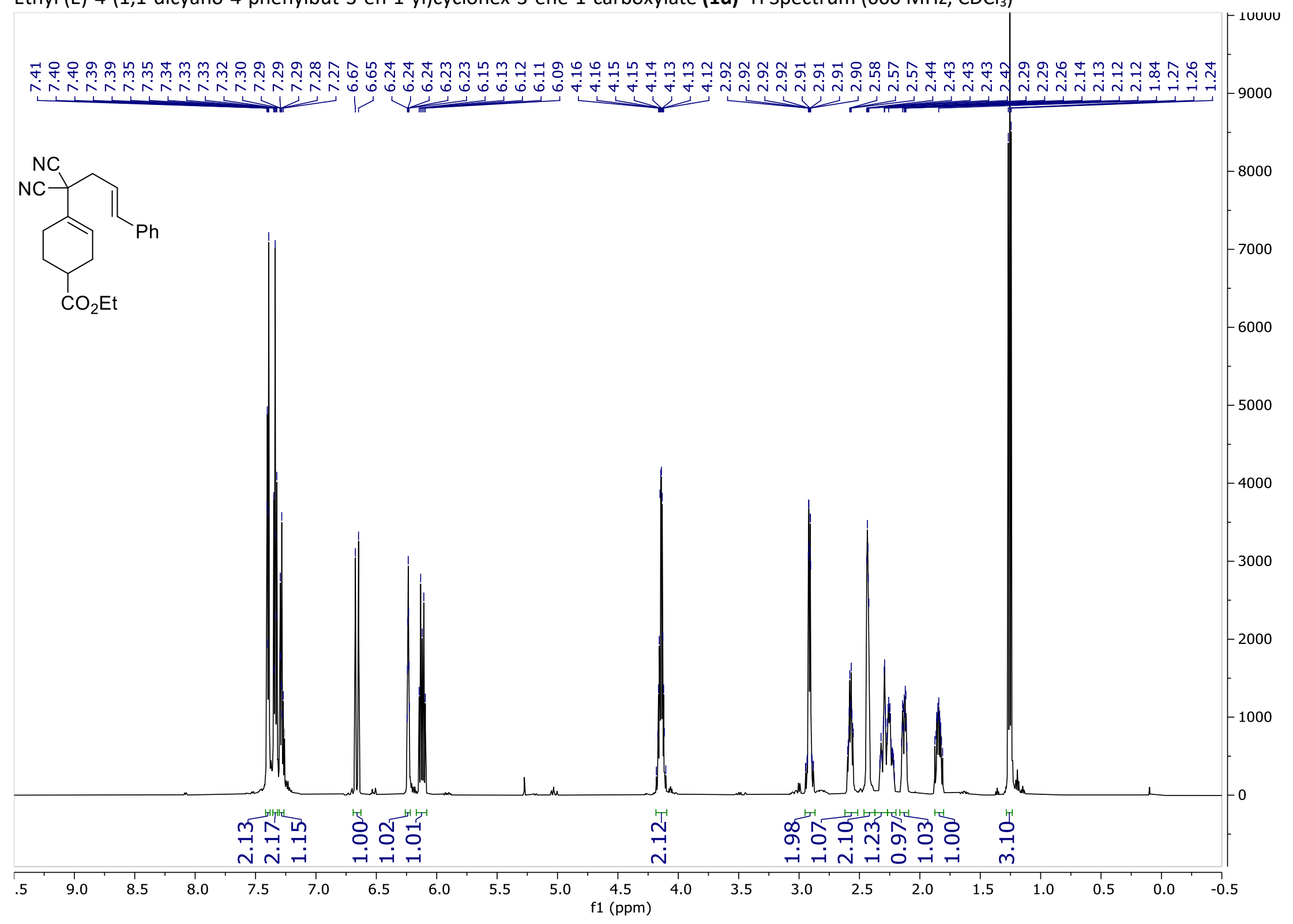

S-69 
ethyl (E)-4-(1,1-dicyano-4-phenylbut-3-en-1-yl)cyclohex-3-ene-1-carboxylate (1d) ${ }^{13} \mathrm{C}$ Spectrum $(151 \mathrm{MHz}, \mathrm{CDCl} 3)$

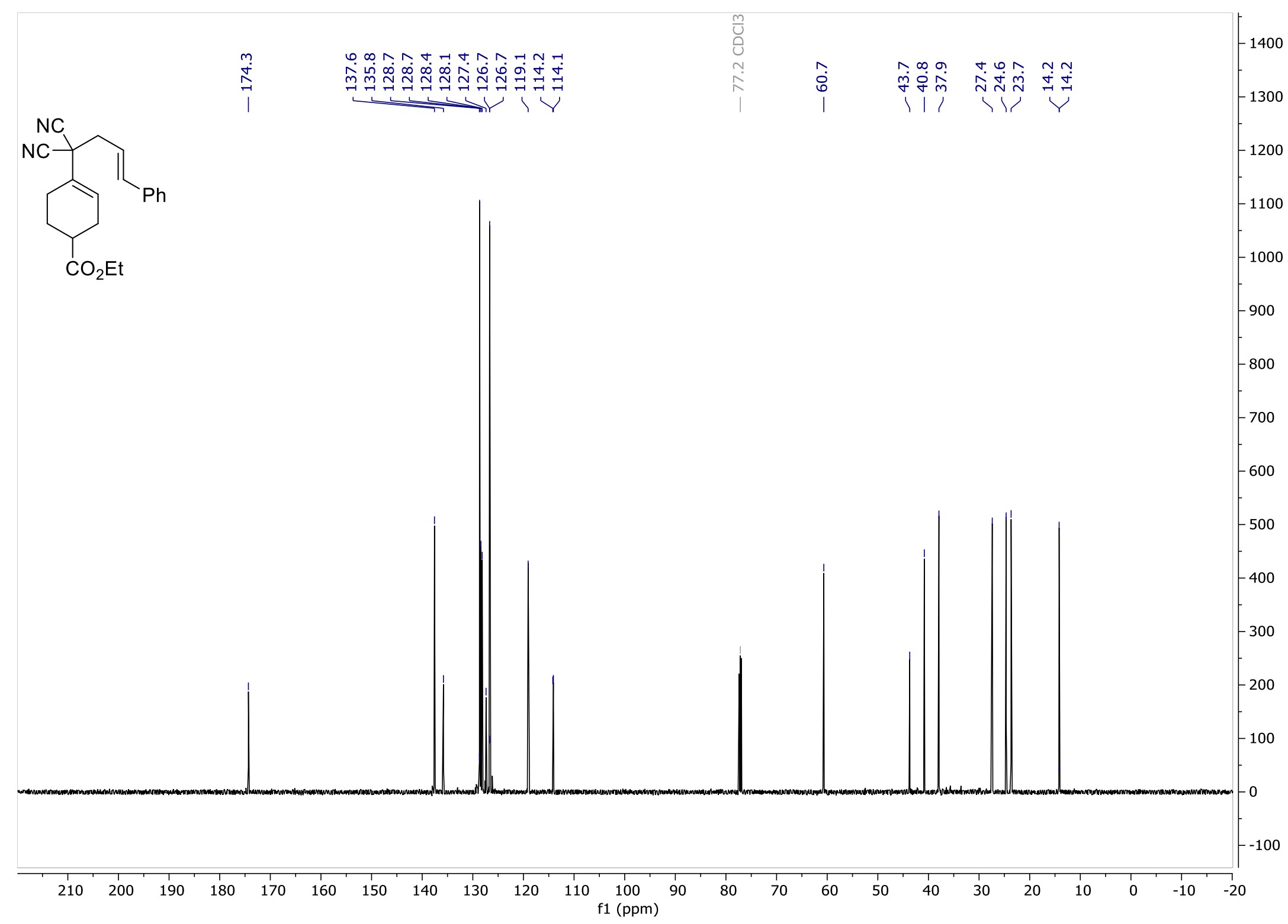


(E)-N-(4-(1,1-dicyano-4-phenylbut-3-en-1-yl)cyclohex-3-en-1-yl)acetamide (1e) ${ }^{1} \mathrm{H}$ Spectrum $\left(600 \mathrm{MHz}, \mathrm{CDCl}_{3}\right)$

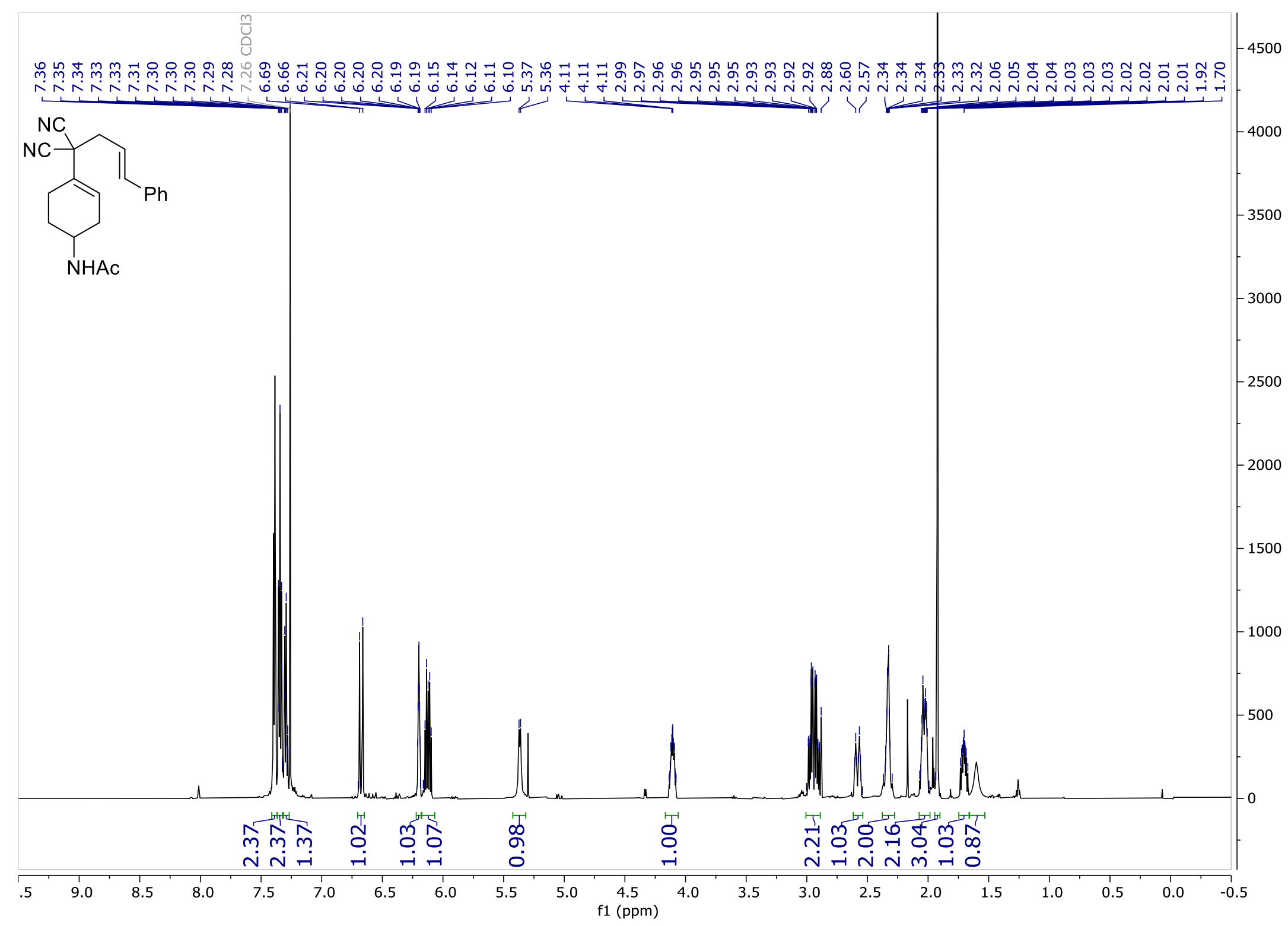

S-71 
(E)-N-(4-(1,1-dicyano-4-phenylbut-3-en-1-yl)cyclohex-3-en-1-yl)acetamide (1e) ${ }^{13} \mathrm{C}$ Spectrum $\left(151 \mathrm{MHz}, \mathrm{CDCl}_{3}\right)$

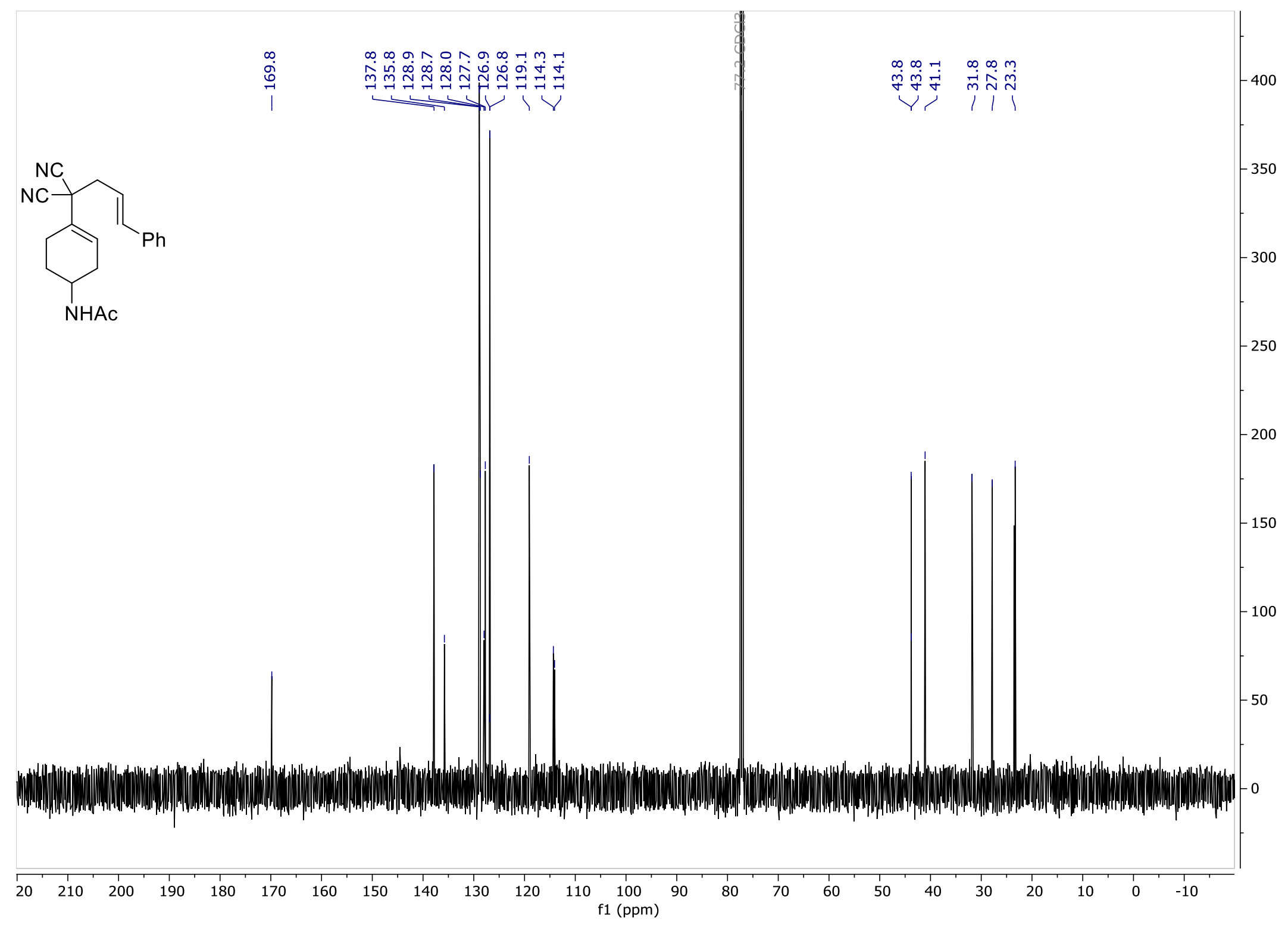


2-cinnamyl-2-(4-(1,3-dioxoisoindolin-2-yl)cyclohex-1-en-1-yl)malononitrile (1f) ${ }^{1} \mathrm{H}$ Spectrum $\left(600 \mathrm{MHz}, \mathrm{CDCl}_{3}\right)$

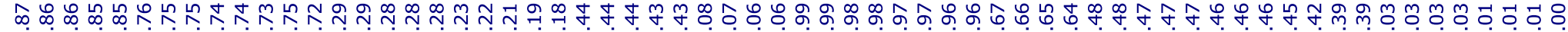

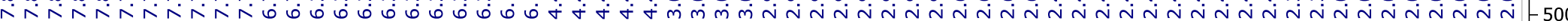

InL

500<smiles>N#CC(C#N)(C/C=C\P)C1=CCC([NH+])CC1</smiles>
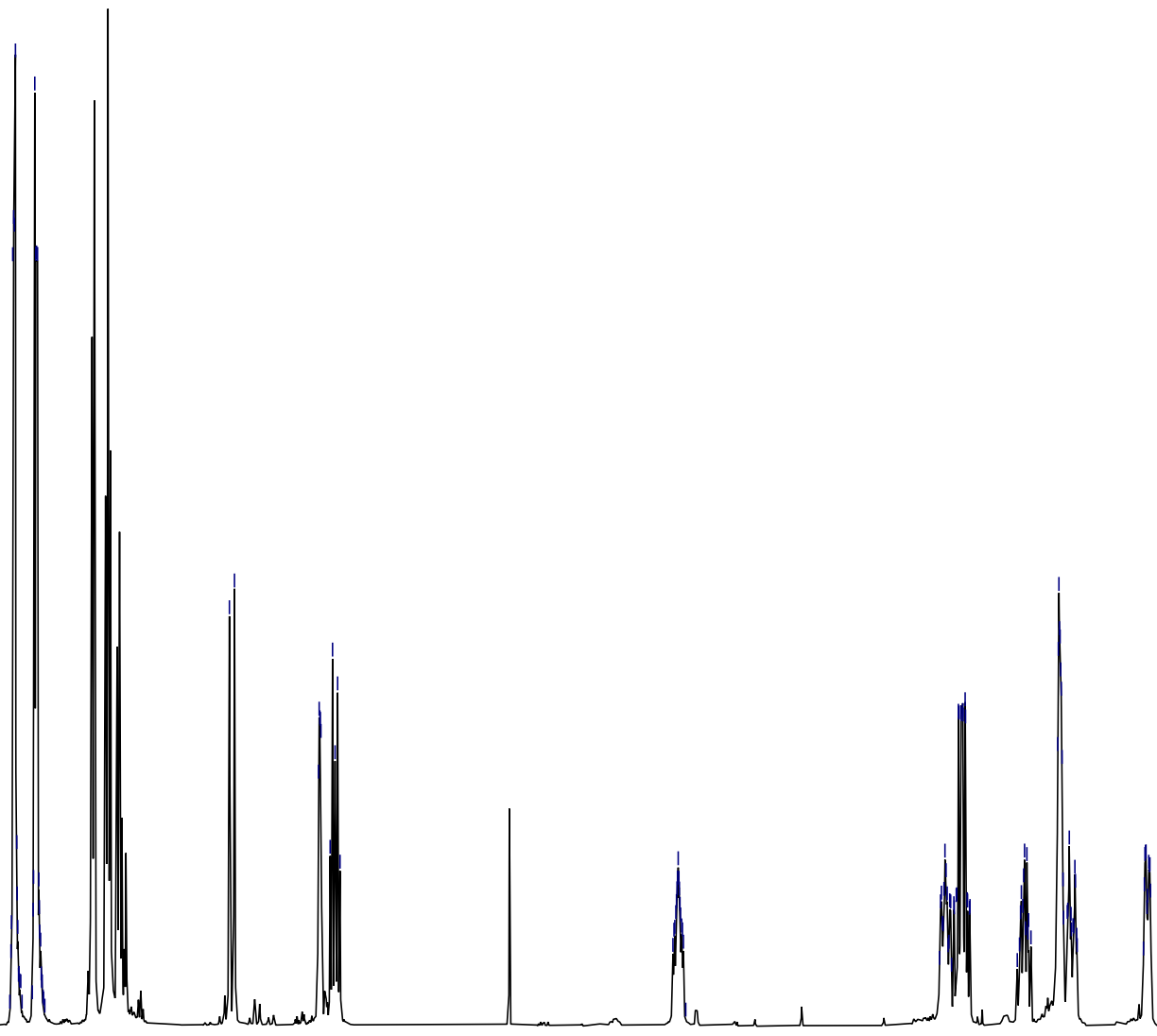

150

100

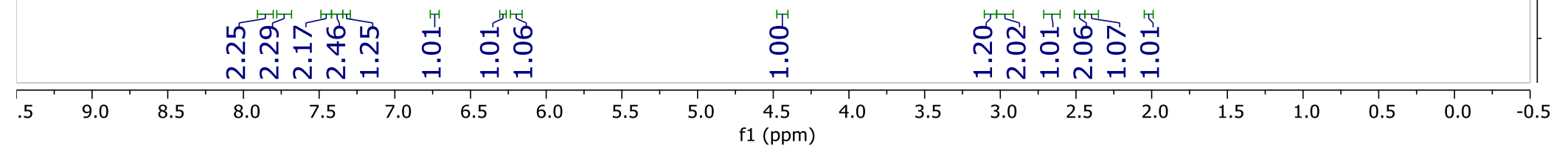


2-cinnamyl-2-(4-(1,3-dioxoisoindolin-2-yl)cyclohex-1-en-1-yl)malononitrile (1f) ${ }^{13} \mathrm{C}$ Spectrum $\left(151 \mathrm{MHz}, \mathrm{CDCl}_{3}\right)$

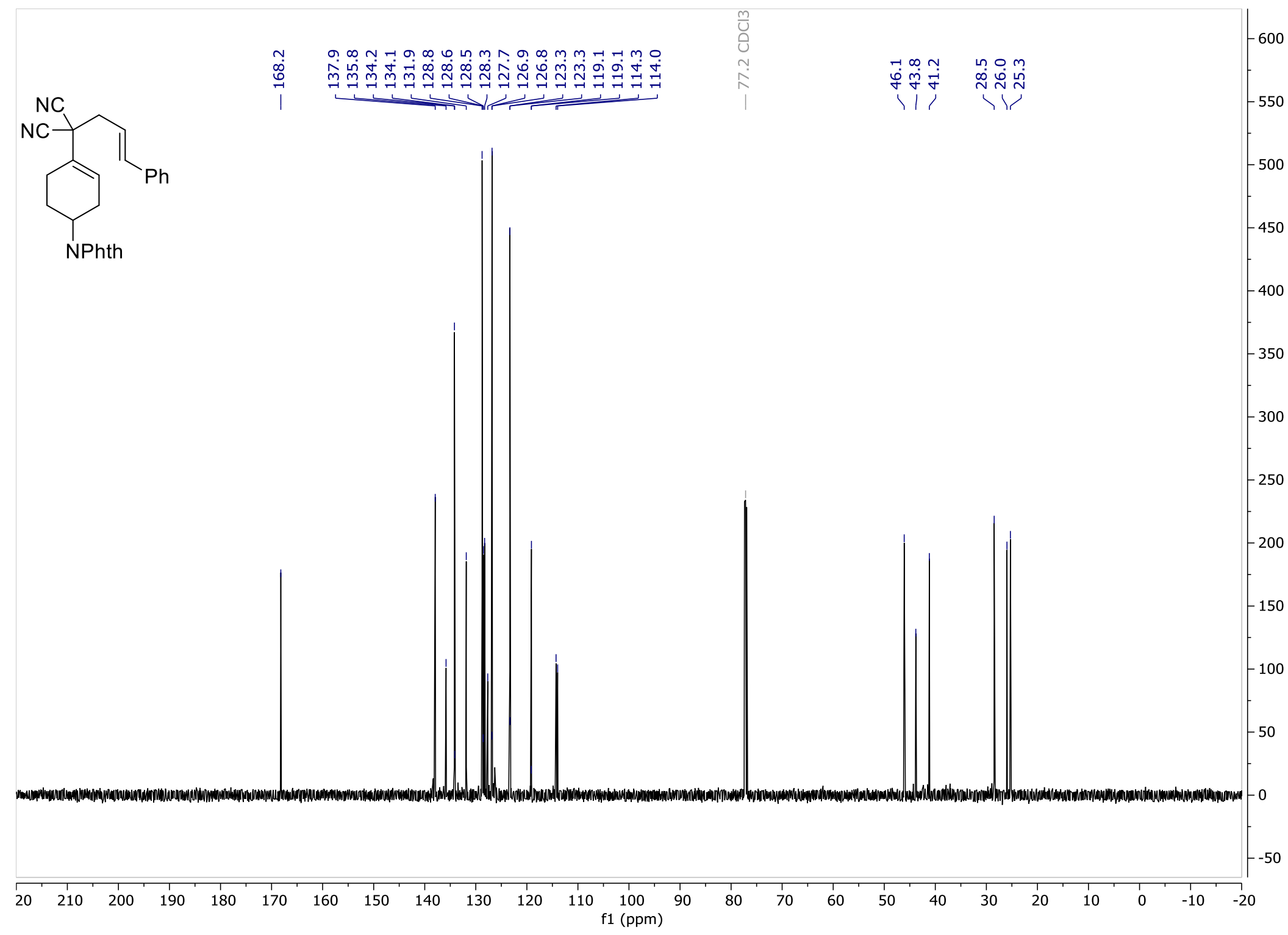


(E)-2-(4-(1,3-dioxoisoindolin-2-yl)cyclohex-1-en-1-yl)-2-(3-(4-fluorophenyl)allyl)malononitrile (1g) ${ }^{1} \mathrm{H}$ Spectrum $\left(600 \mathrm{MHz}, \mathrm{CDCl}_{3}\right)$

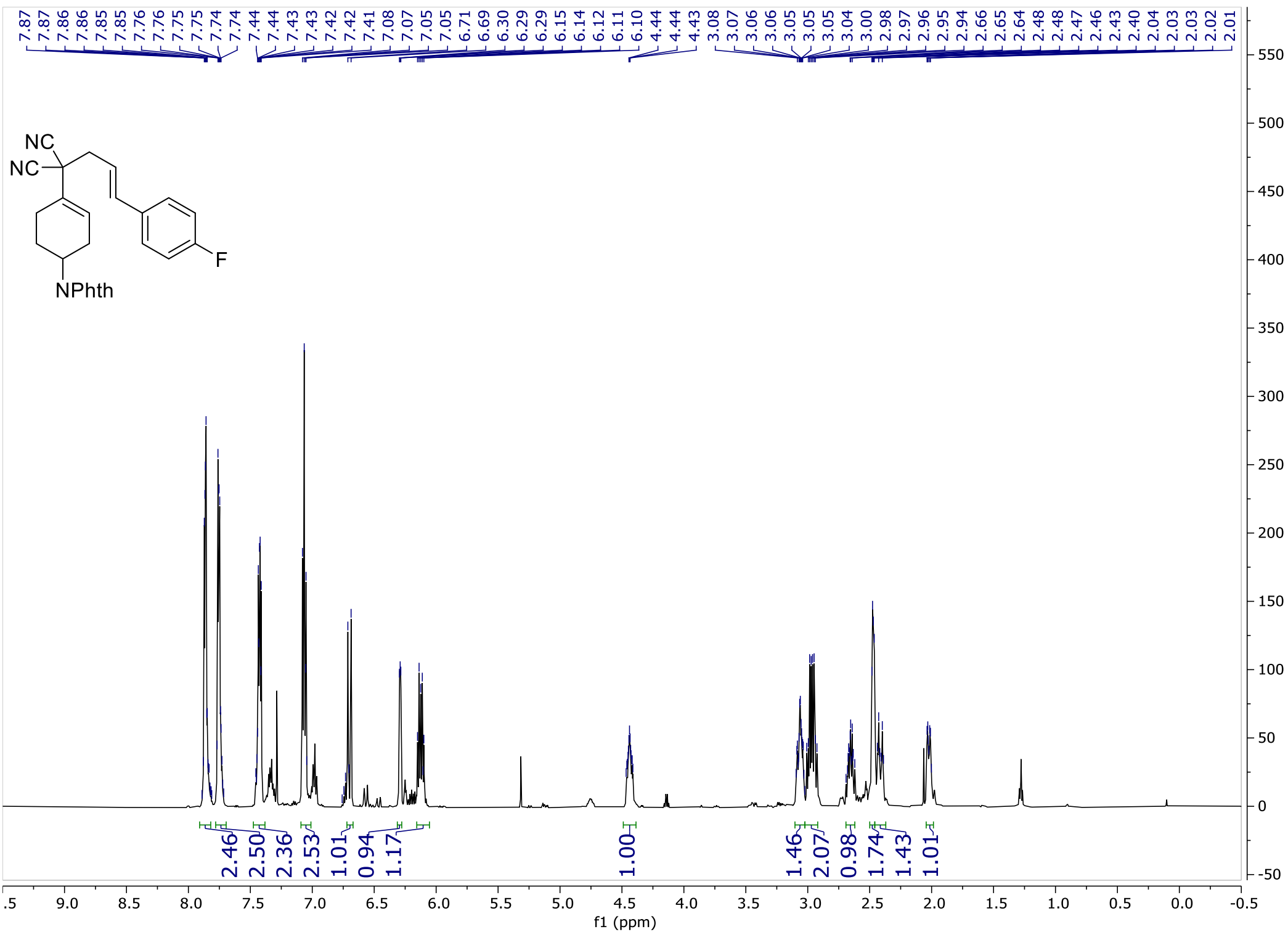


(E)-2-(4-(1,3-dioxoisoindolin-2-yl)cyclohex-1-en-1-yl)-2-(3-(4-fluorophenyl)allyl)malononitrile (1g) ${ }^{13} \mathrm{C}$ Spectrum $\left(151 \mathrm{MHz}, \mathrm{CDCl}_{3}\right)$

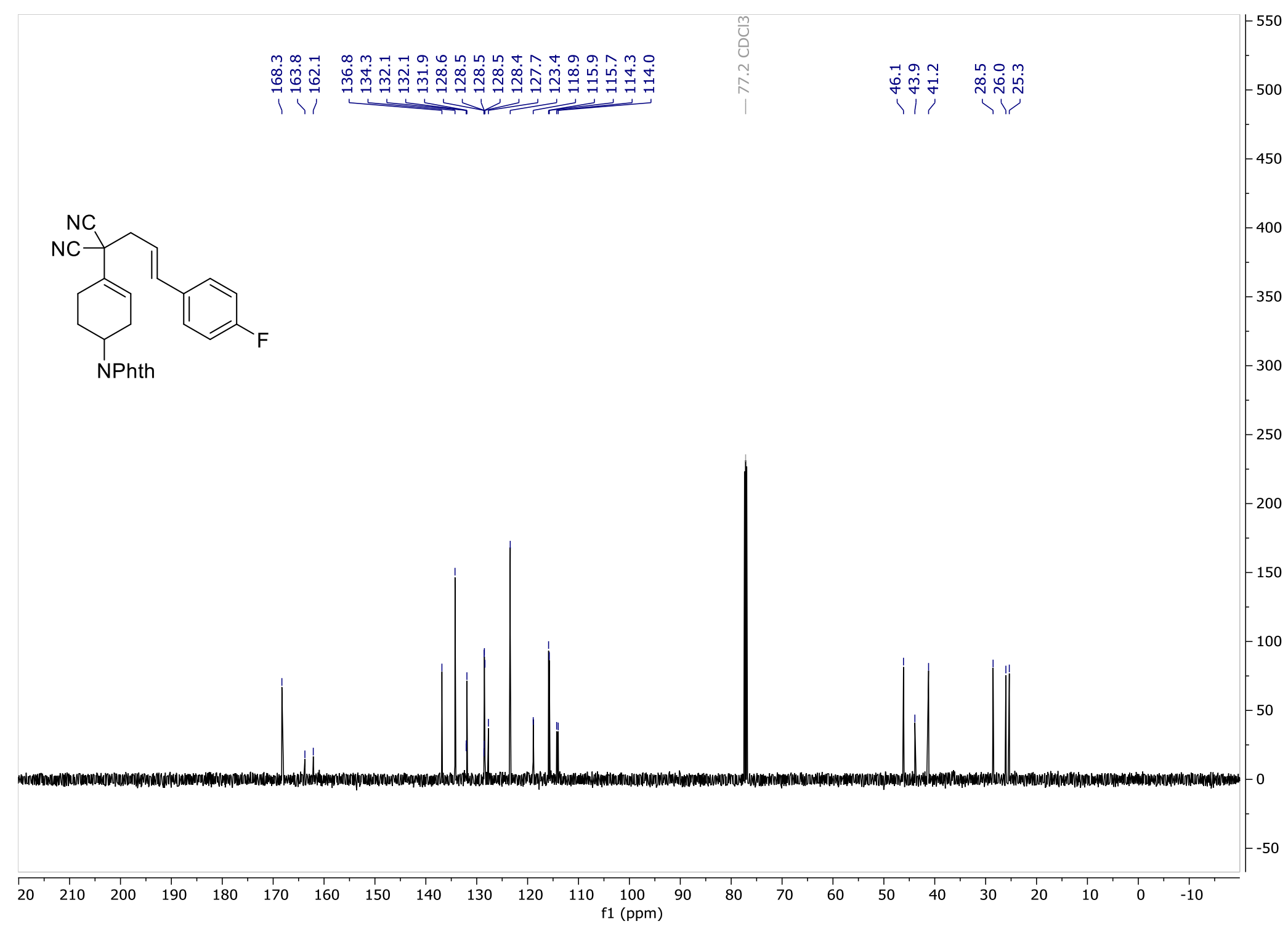


(E)-2-(4-(1,3-dioxoisoindolin-2-yl)cyclohex-1-en-1-yl)-2-(3-(4-fluorophenyl)allyl)malononitrile $(1 \mathrm{~g}){ }^{19} \mathrm{~F}$ Spectrum $\left(565 \mathrm{MHz}^{\mathrm{CDCl}}{ }_{3}\right)$

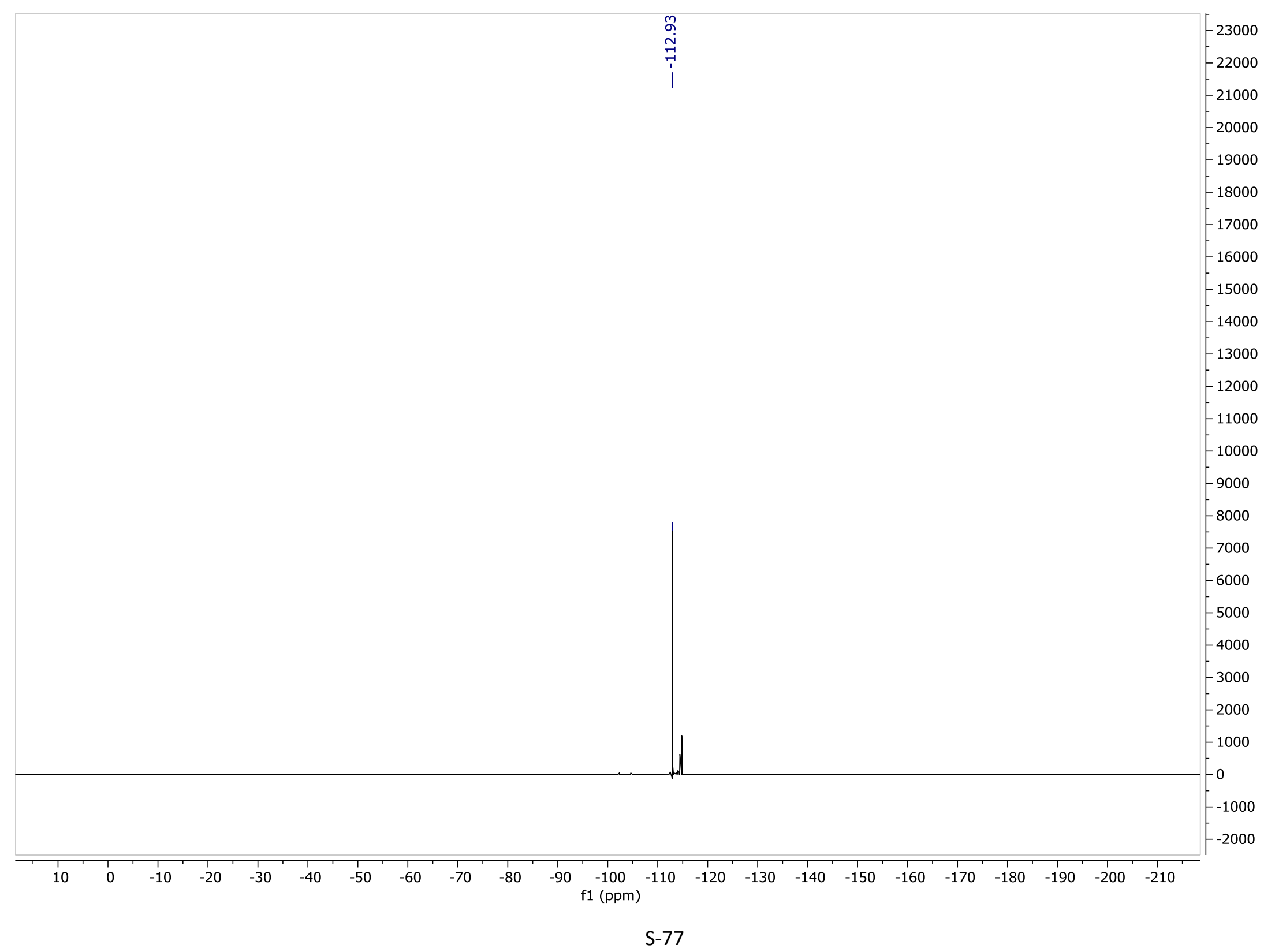


(E)-2-(4-(1,3-dioxoisoindolin-2-yl)cyclohex-1-en-1-yl)-2-(3-(4-fluorophenyl)allyl)malononitrile (1g) HMBC Spectrum

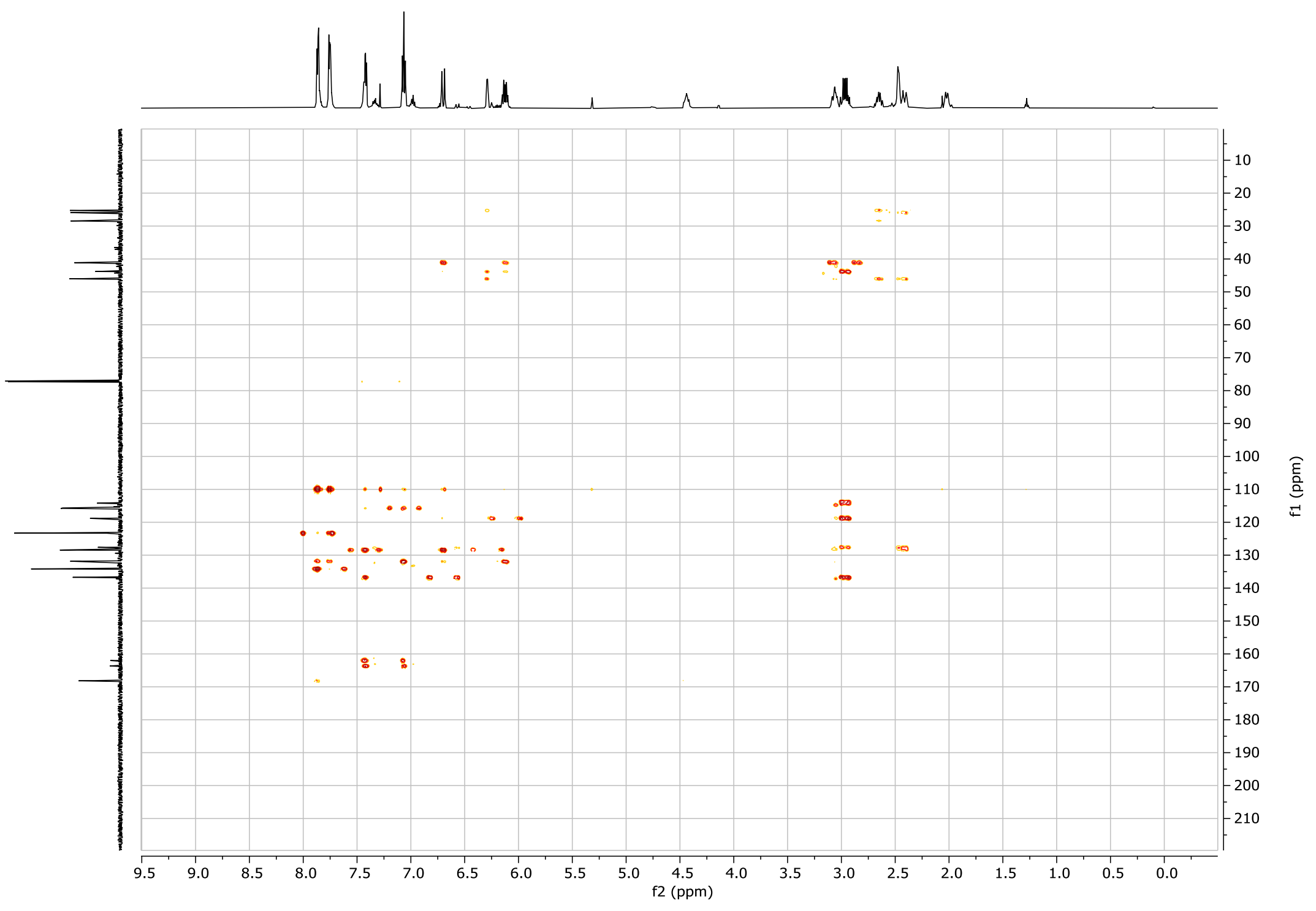


(E)-2-(4-(1,3-dioxoisoindolin-2-yl)cyclohex-1-en-1-yl)-2-(3-(2-fluorophenyl)allyl)malononitrile (1h) ${ }^{1} \mathrm{H}$ Spectrum $\left(600 \mathrm{MHz}, \mathrm{CDCl}_{3}\right)$

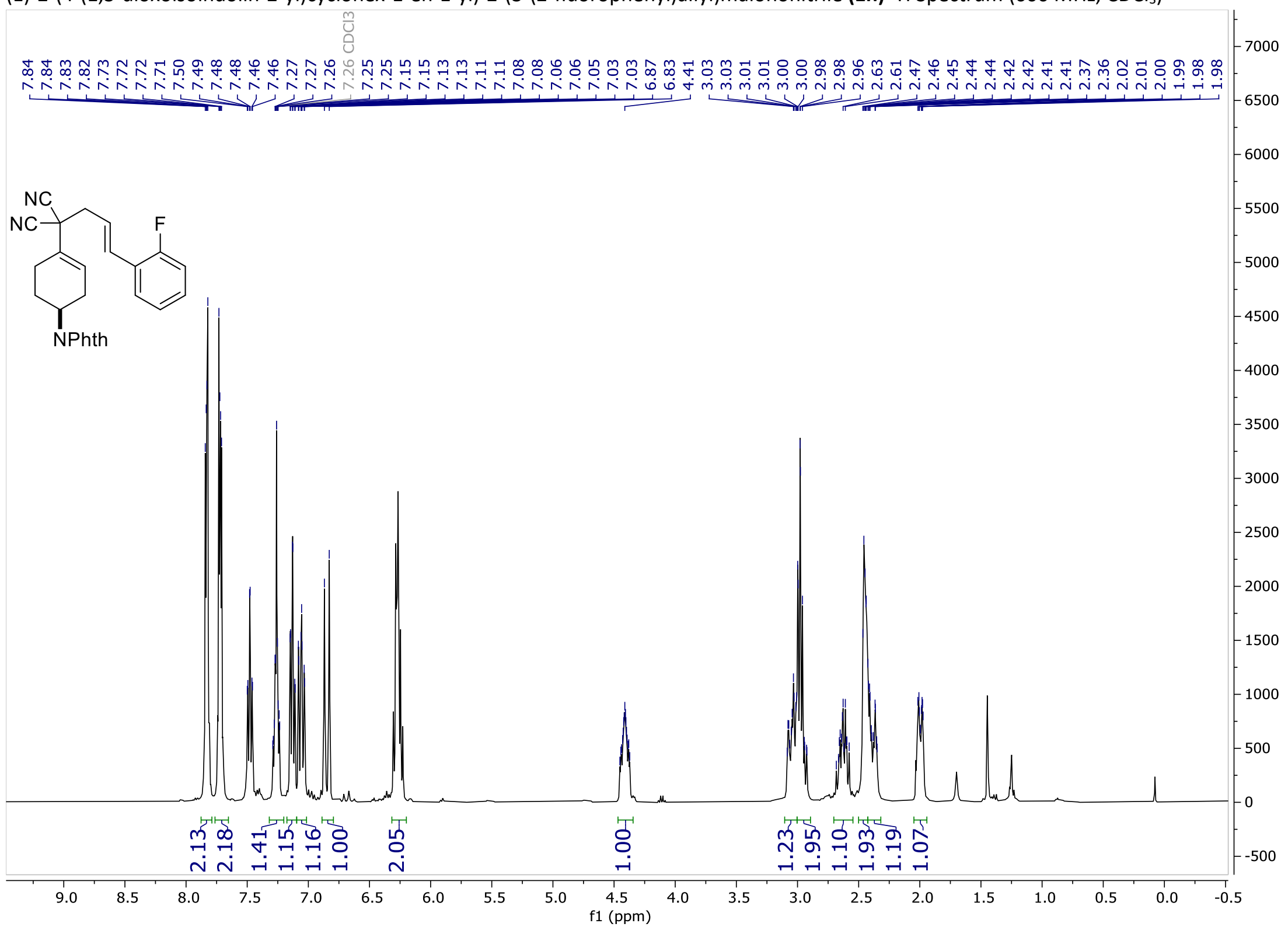


(S,E)-2-(4-(1,3-dioxoisoindolin-2-yl)cyclohex-1-en-1-yl)-2-(3-(2-fluorophenyl)allyl)malononitrile (1h) ${ }^{13} \mathrm{C}$ Spectrum $\left(151 \mathrm{MHz}, \mathrm{CDCl}_{3}\right)$

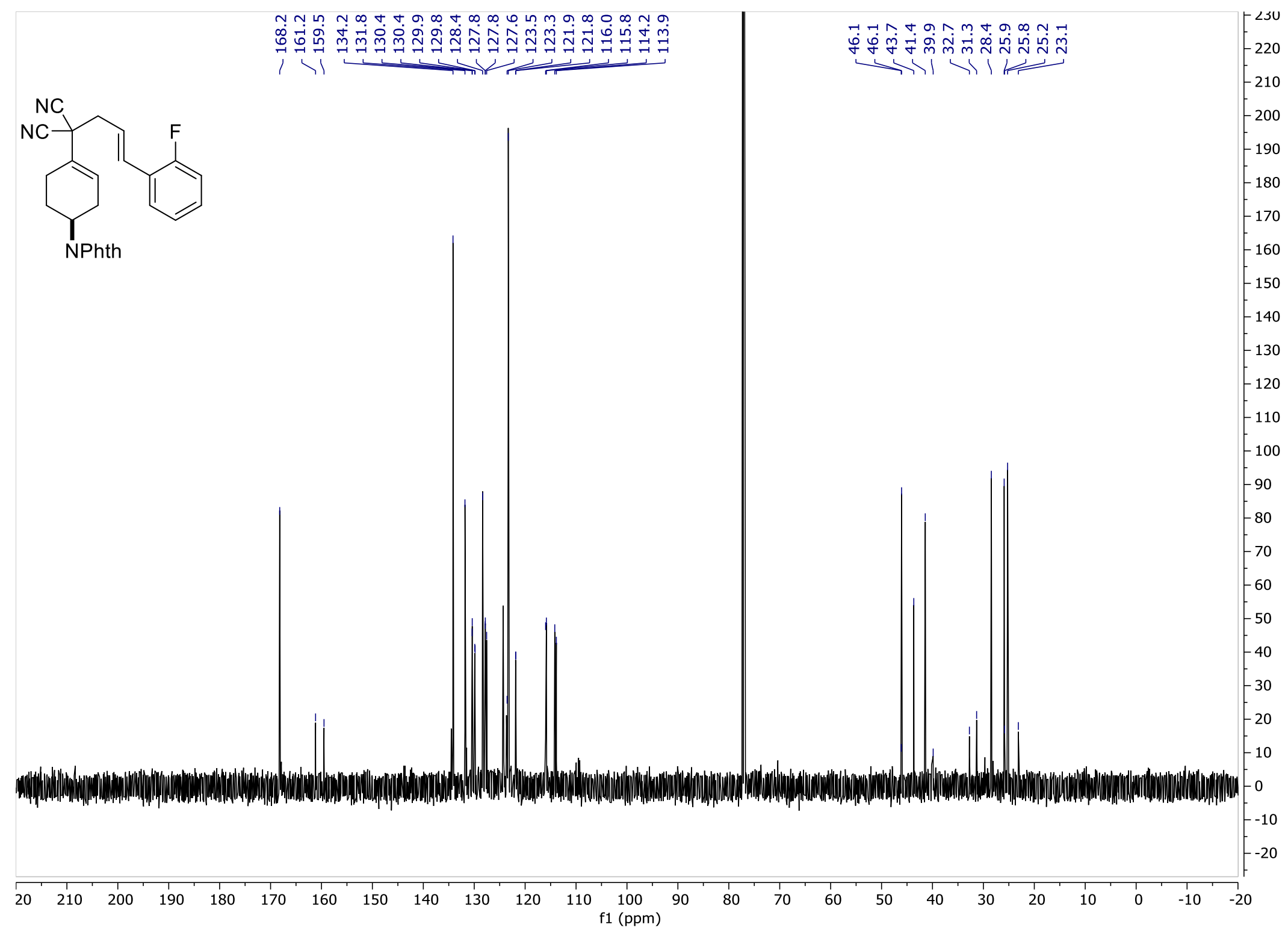


(S,E)-2-(4-(1,3-dioxoisoindolin-2-yl)cyclohex-1-en-1-yl)-2-(3-(2-fluorophenyl)allyl)malononitrile (1h) ${ }^{19} \mathrm{~F}$ Spectrum $\left(565 \mathrm{MHz}^{\mathrm{C}} \mathrm{CDCl}_{3}\right)$

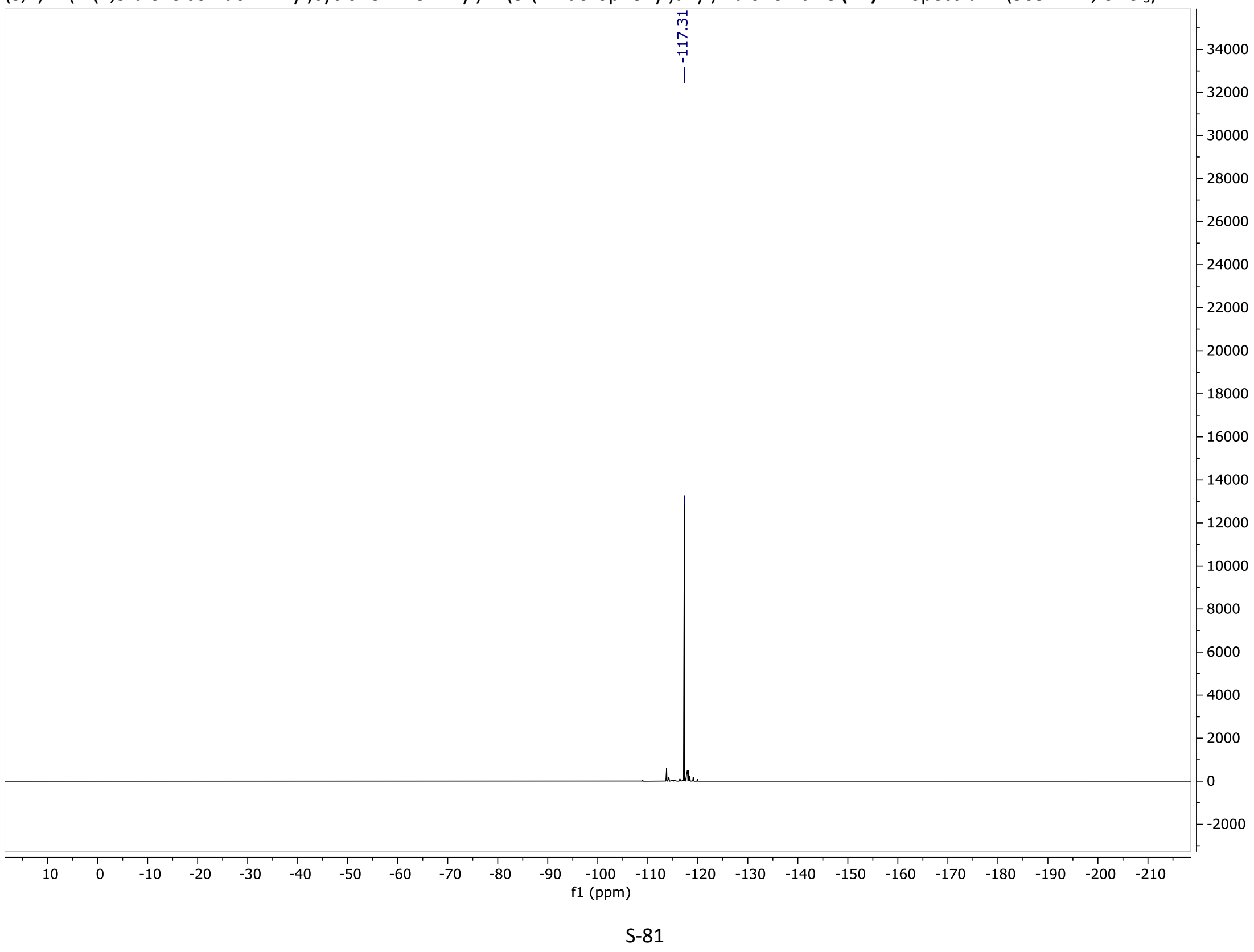


(S,E)-2-(4-(1,3-dioxoisoindolin-2-yl)cyclohex-1-en-1-yl)-2-(3-(2-fluorophenyl)allyl)malononitrile (1h) HMBC Spectrum

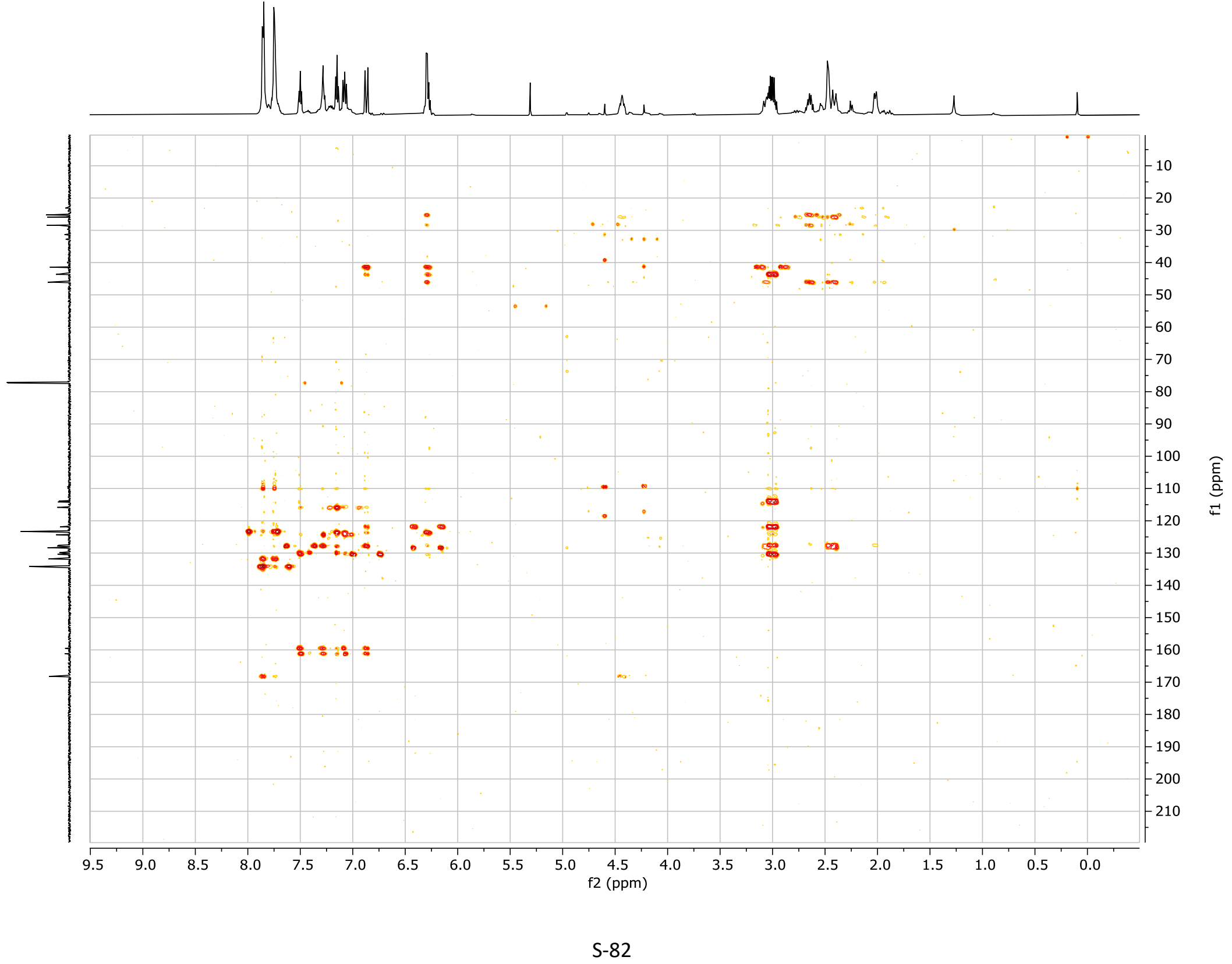


S,E)-2-(4-(1,3-dioxoisoindolin-2-yl)cyclohex-1-en-1-yl)-2-(3-(4-methoxyphenyl)allyl)malononitrile (1i) ${ }^{1} \mathrm{H}$ Spectrum $(600 \mathrm{MHz} \mathrm{CDCl} 3$ )

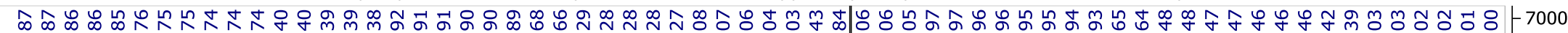

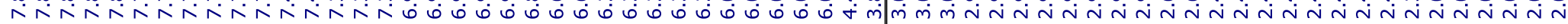

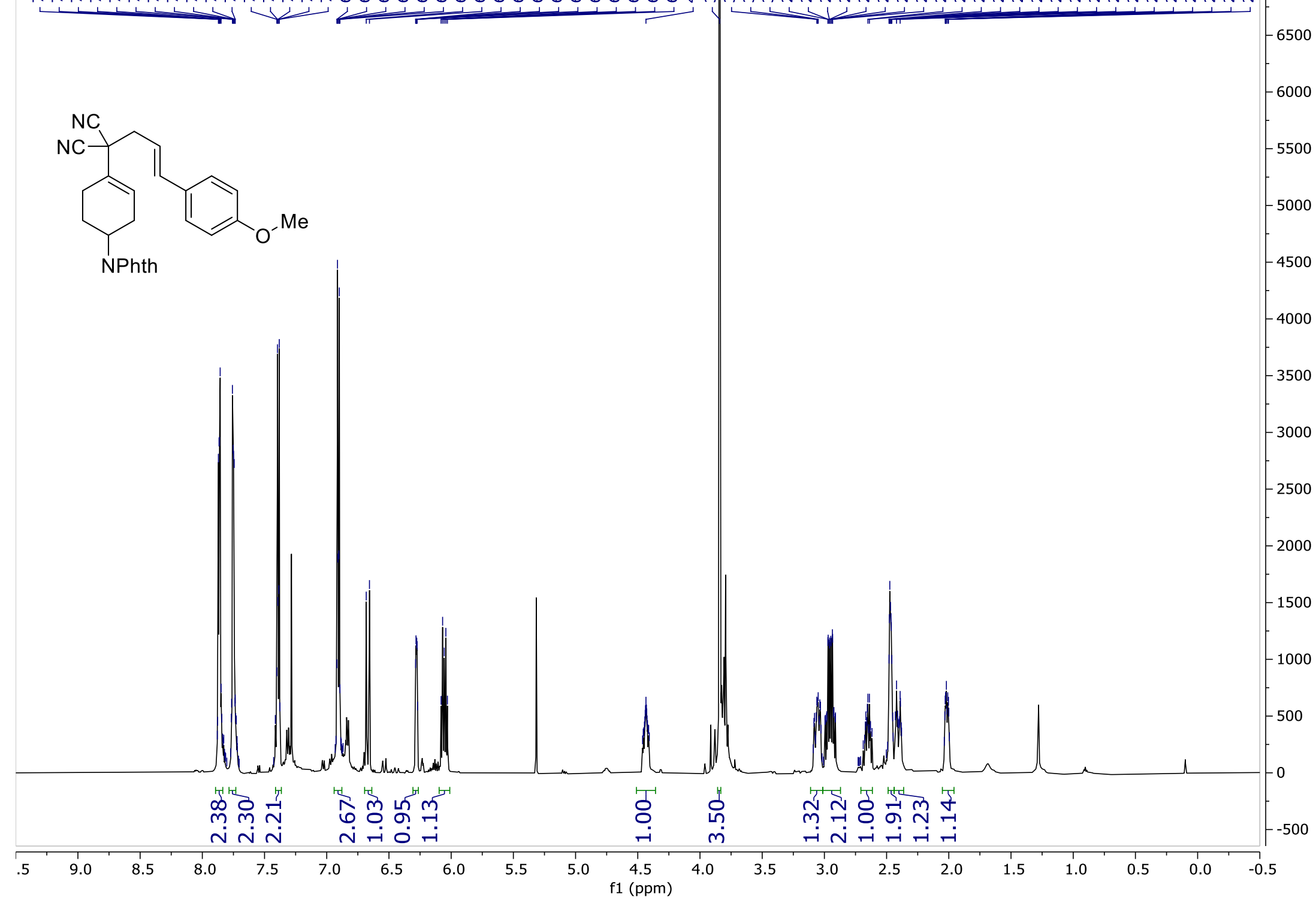


(S,E)-2-(4-(1,3-dioxoisoindolin-2-yl)cyclohex-1-en-1-yl)-2-(3-(4-methoxyphenyl)allyl)malononitrile (1i) ${ }^{13} \mathrm{C}$ Spectrum $\left(595 \mathrm{MHz}^{\mathrm{CDCl}}{ }_{3}\right)$

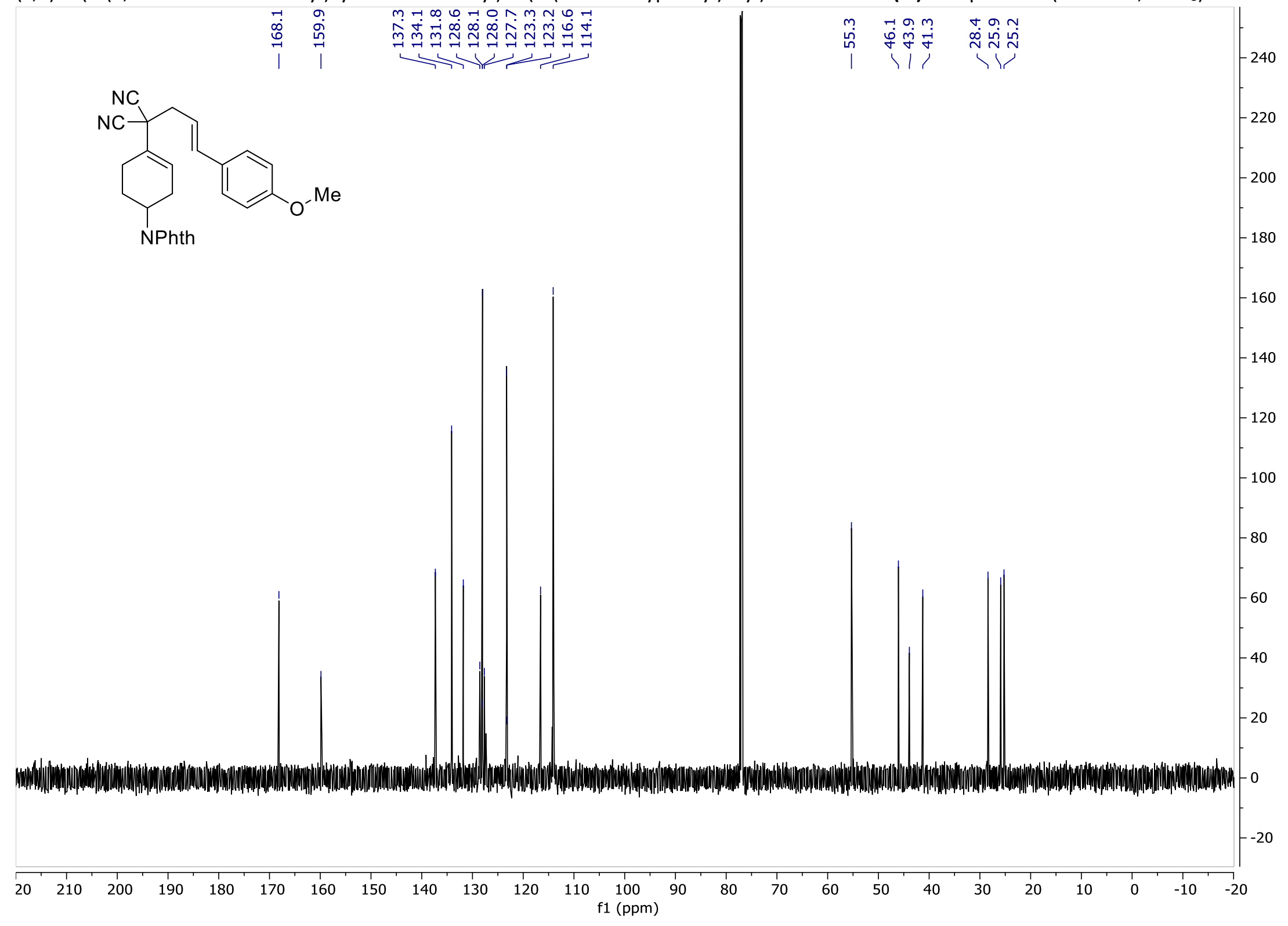


(E)-2-(3-(4-(1,3-dioxolan-2-yl)phenyl)allyl)-2-(4-(1,3-dioxoisoindolin-2-yl)cyclohex-1-en-1-yl)malononitrile (1j) ${ }^{1} \mathrm{H} \mathrm{Spectrum} \mathrm{(600} \mathrm{MHz,} \mathrm{CDCl} 3$ )

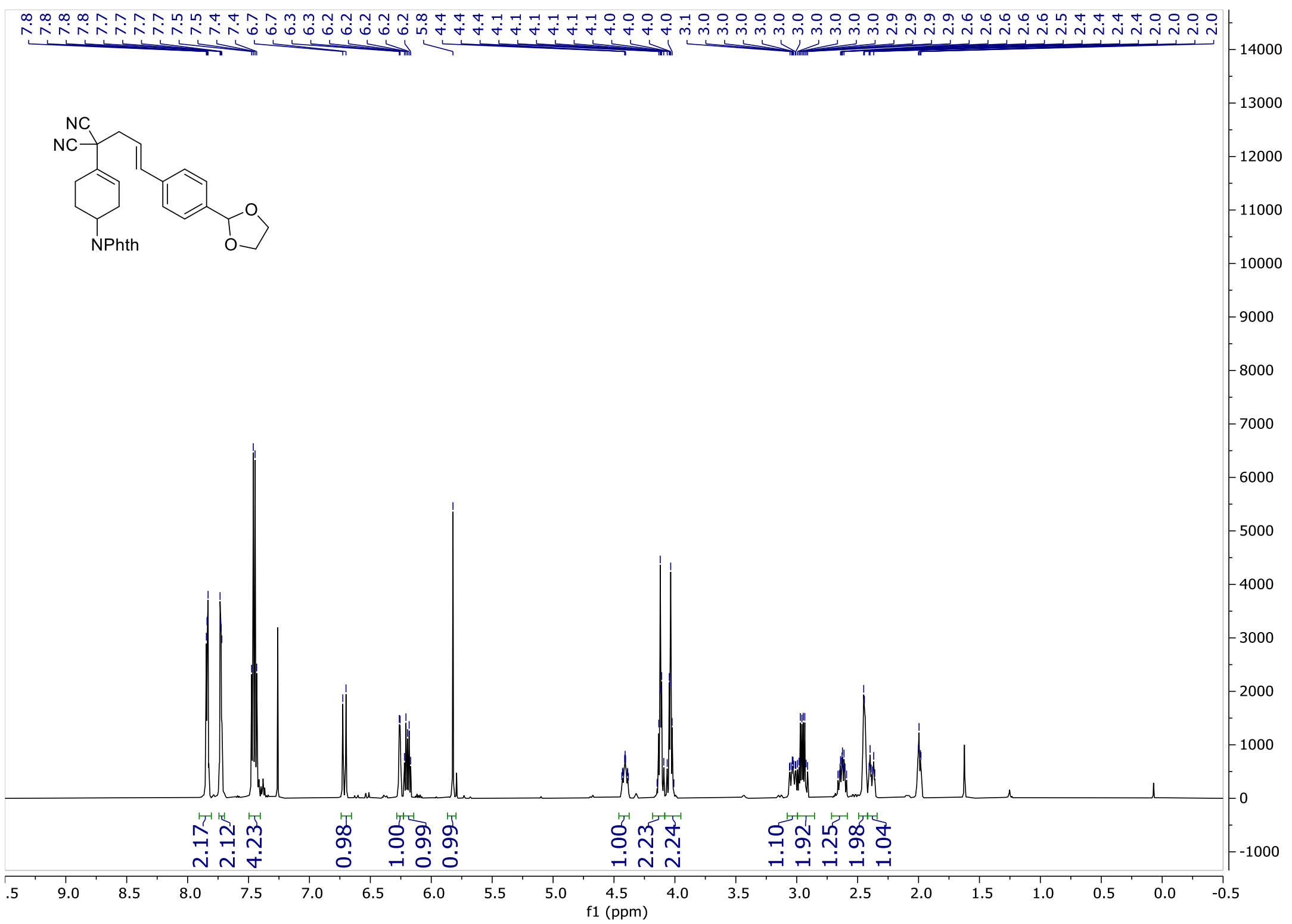


(E)-2-(3-(4-(1,3-dioxolan-2-yl)phenyl)allyl)-2-(4-(1,3-dioxoisoindolin-2-yl)cyclohex-1-en-1-yl)malononitrile (1j) ${ }^{13} \mathrm{C}$ Spectrum

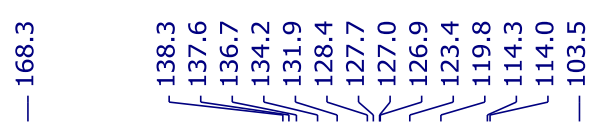
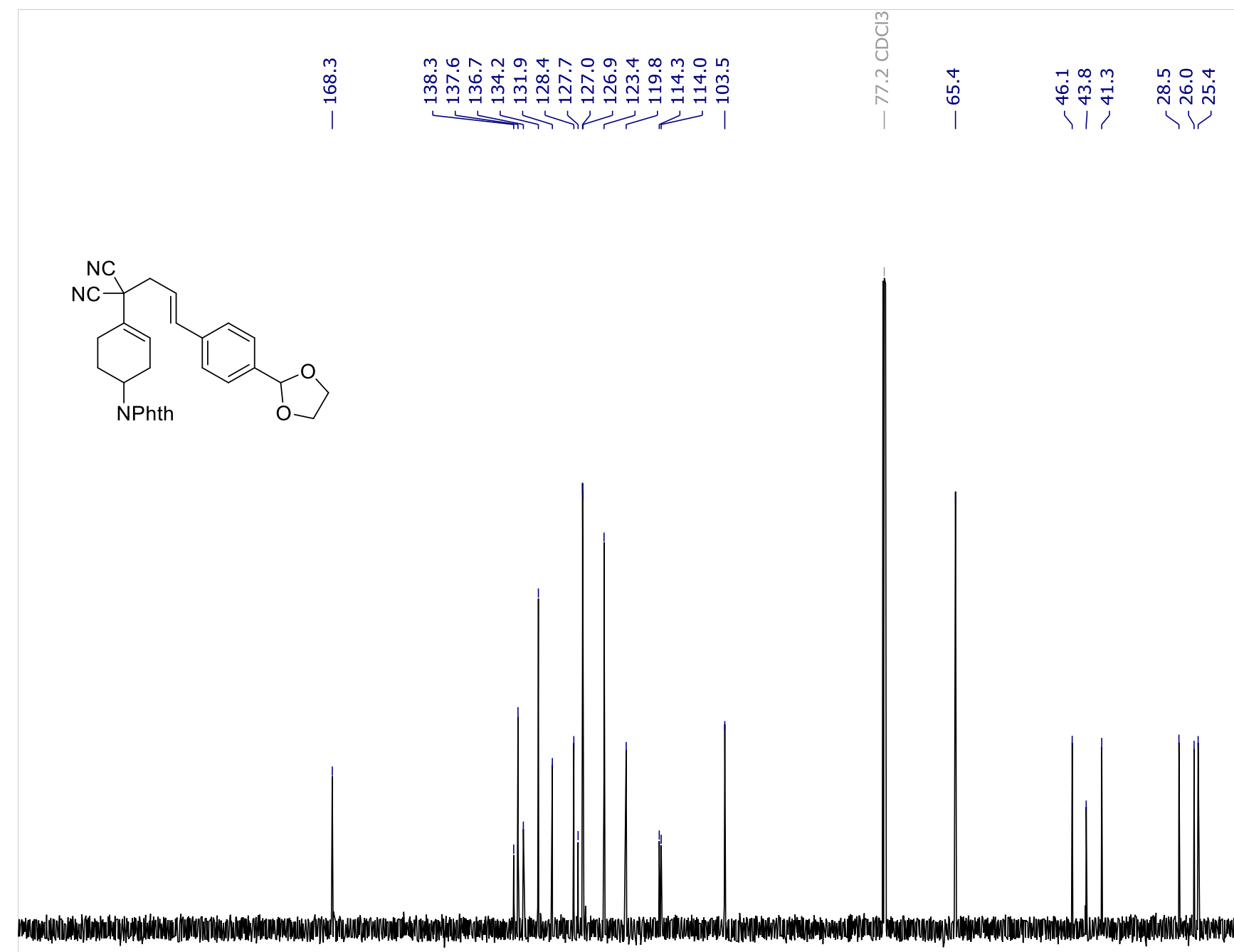

S-86 
2-((1S,2R,4S)-4-(1,3-dioxoisoindolin-2-yl)-2-((R)-1-phenylallyl)cyclohexyl)malononitrile $(3 f){ }^{13} \mathrm{H}$ Spectrum $\left(600 \mathrm{MHz} \mathrm{CDCl}_{3}\right)$

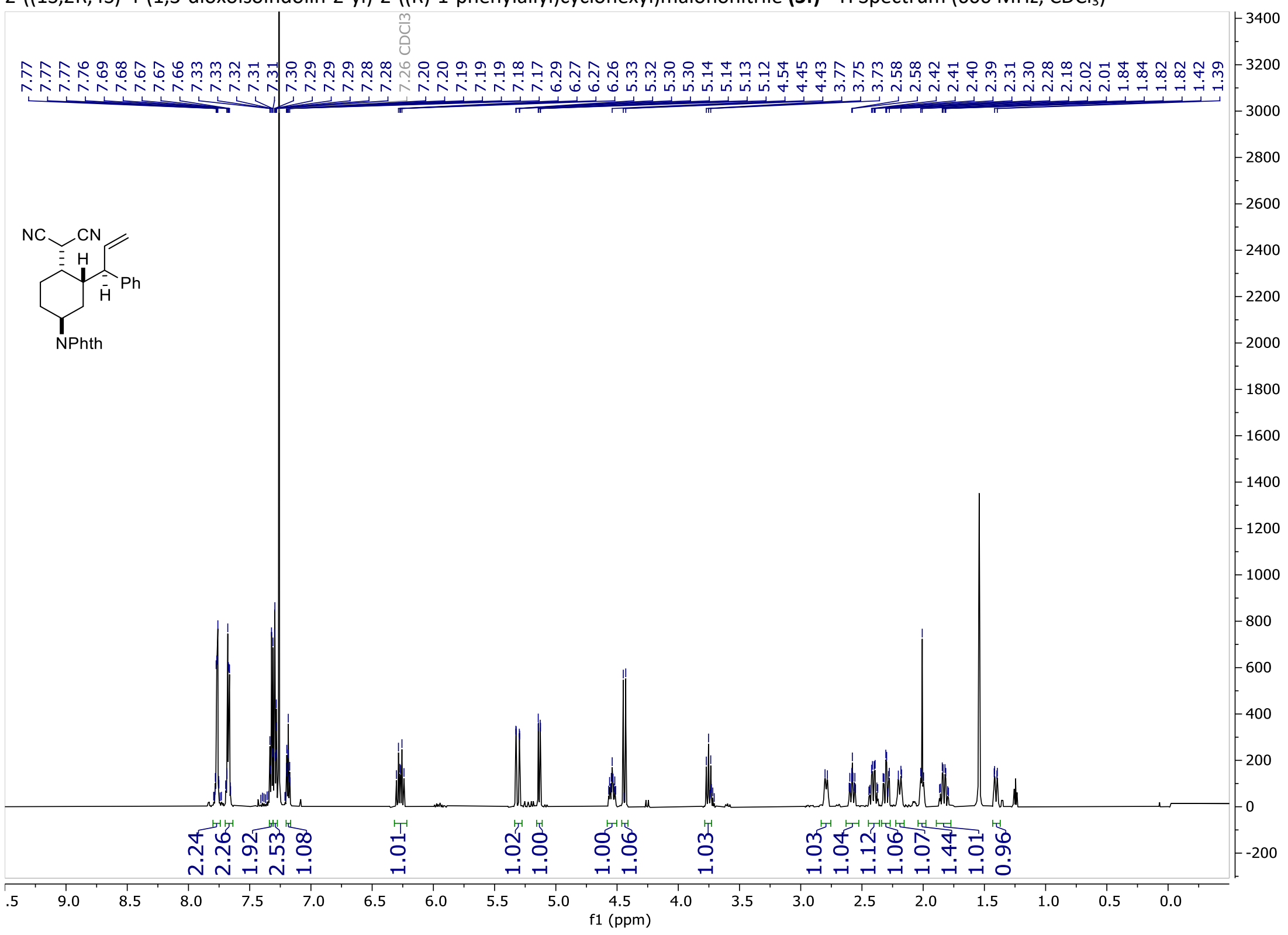


2-((1S,2R,4S)-4-(1,3-dioxoisoindolin-2-yl)-2-((R)-1-phenylallyl)cyclohexyl)malononitrile (3f) ${ }^{13} \mathrm{C}$ Spectrum $\left(151 \mathrm{MHz}, \mathrm{CDCl}_{3}\right)$

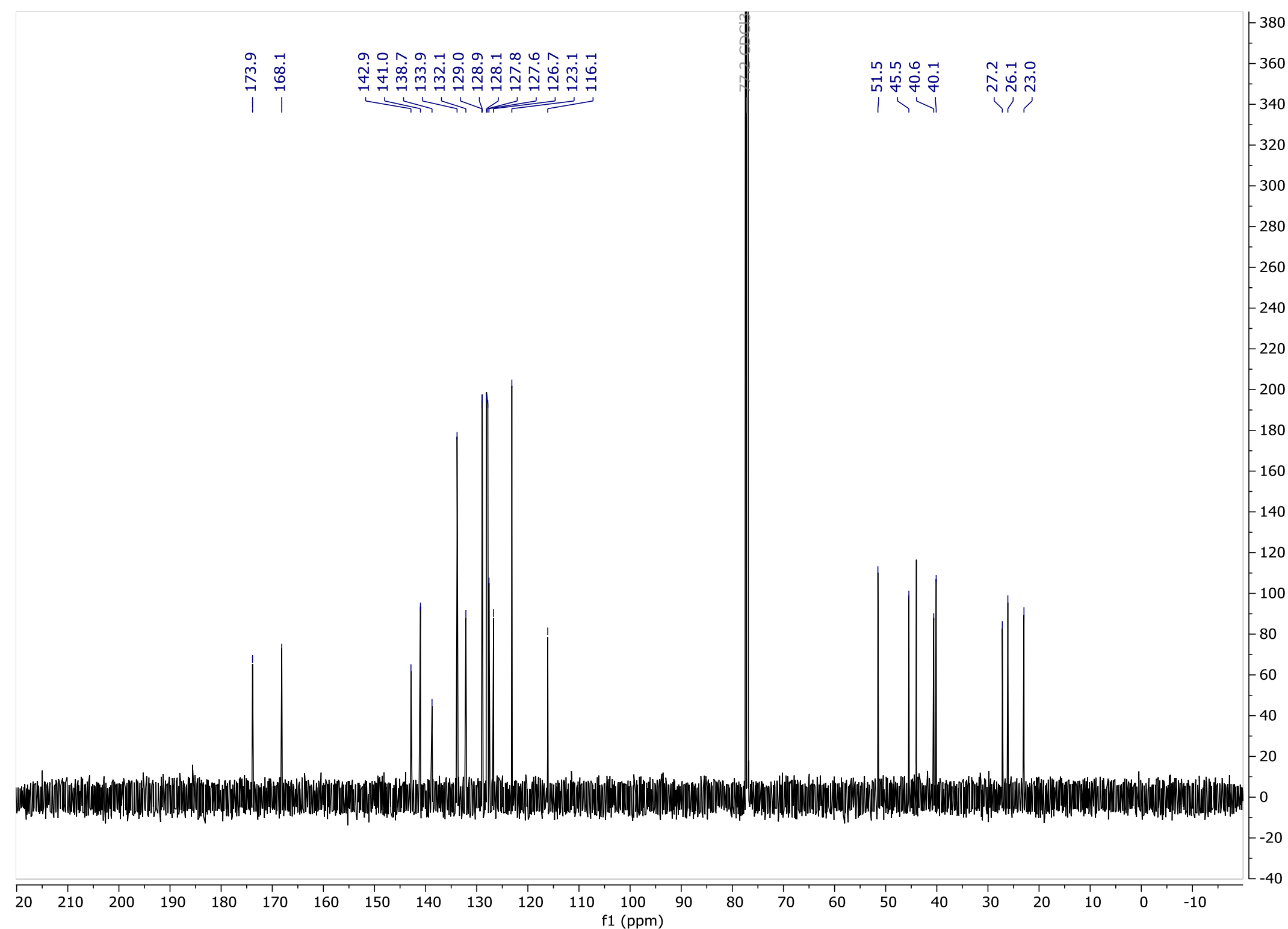


2-((1S,2R,4S)-4-(1,3-dioxoisoindolin-2-yl)-2-((R)-1-phenylallyl)cyclohexyl)malononitrile (3f) ${ }^{1} \mathrm{H}$ Spectrum (11.9:1 d.r.) $\left(600 \mathrm{MHz}, \mathrm{CDCl}_{3}\right)$

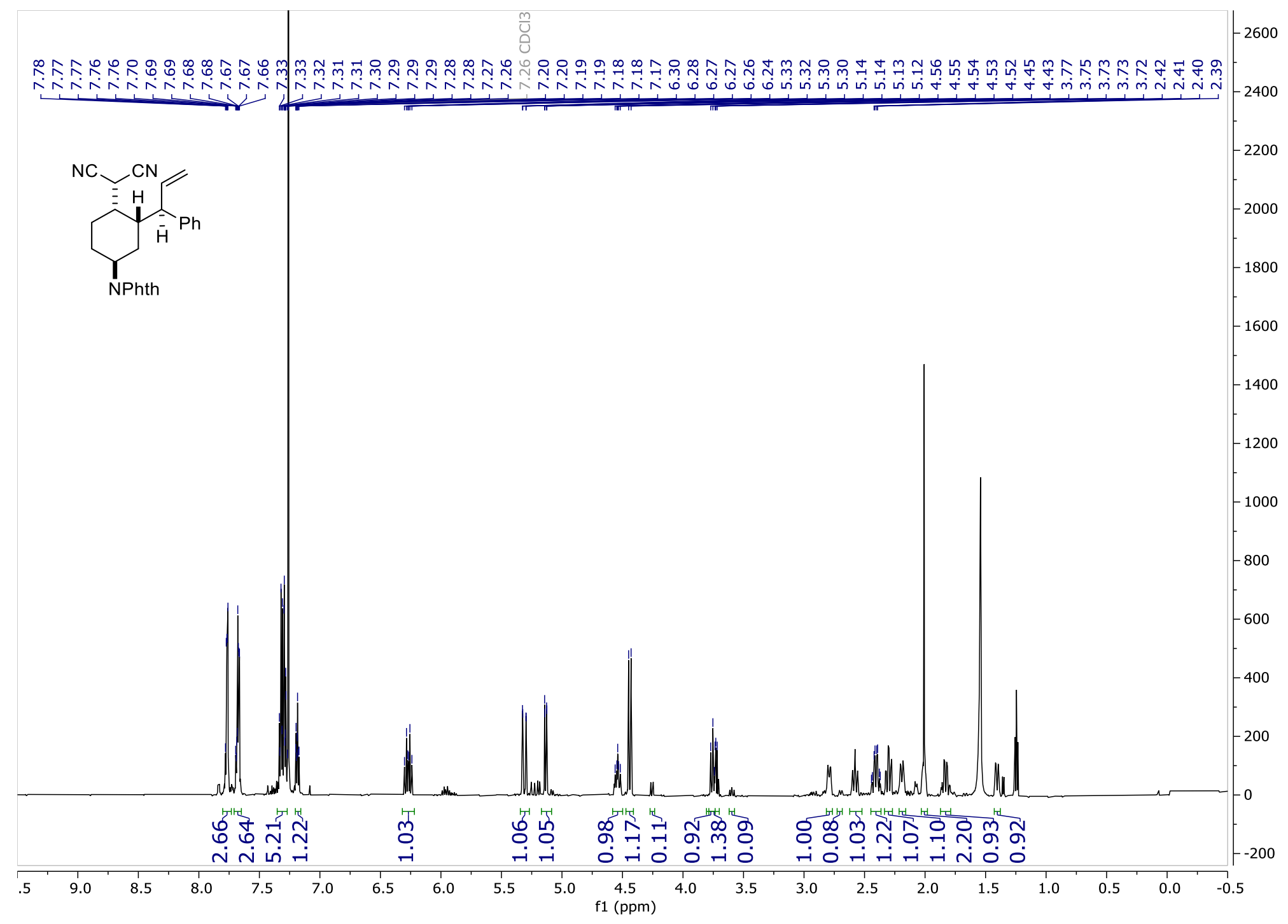


morpholino((1S,2S,4S)-4-phenyl-2-((R)-1-phenylallyl)cyclohexyl)methanone (4a) 13.4:1 d. ${ }^{1}{ }^{1} \mathrm{H}$ Spectrum $\left(600 \mathrm{MHz}, \mathrm{CDCl}_{3}\right)$

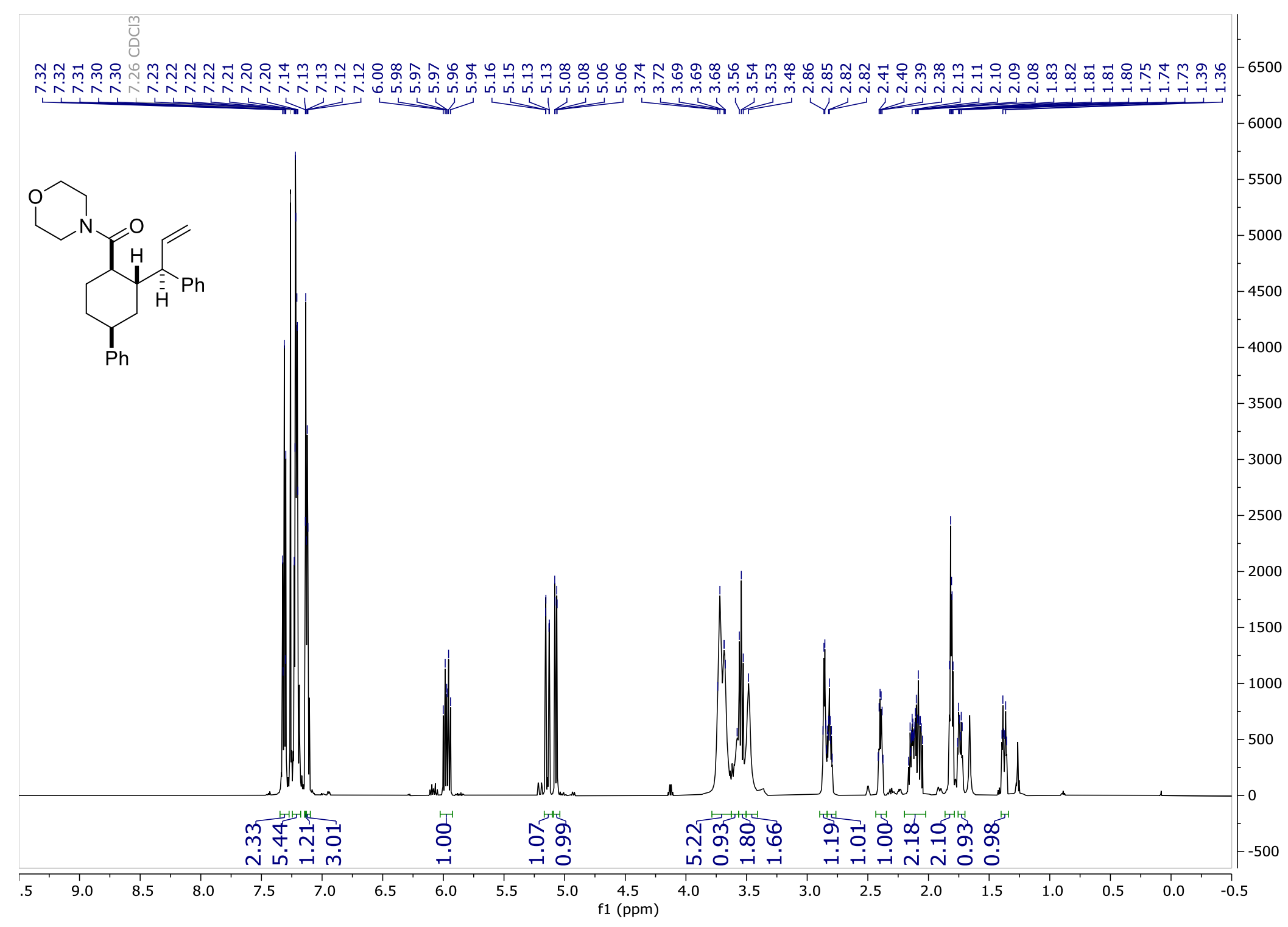




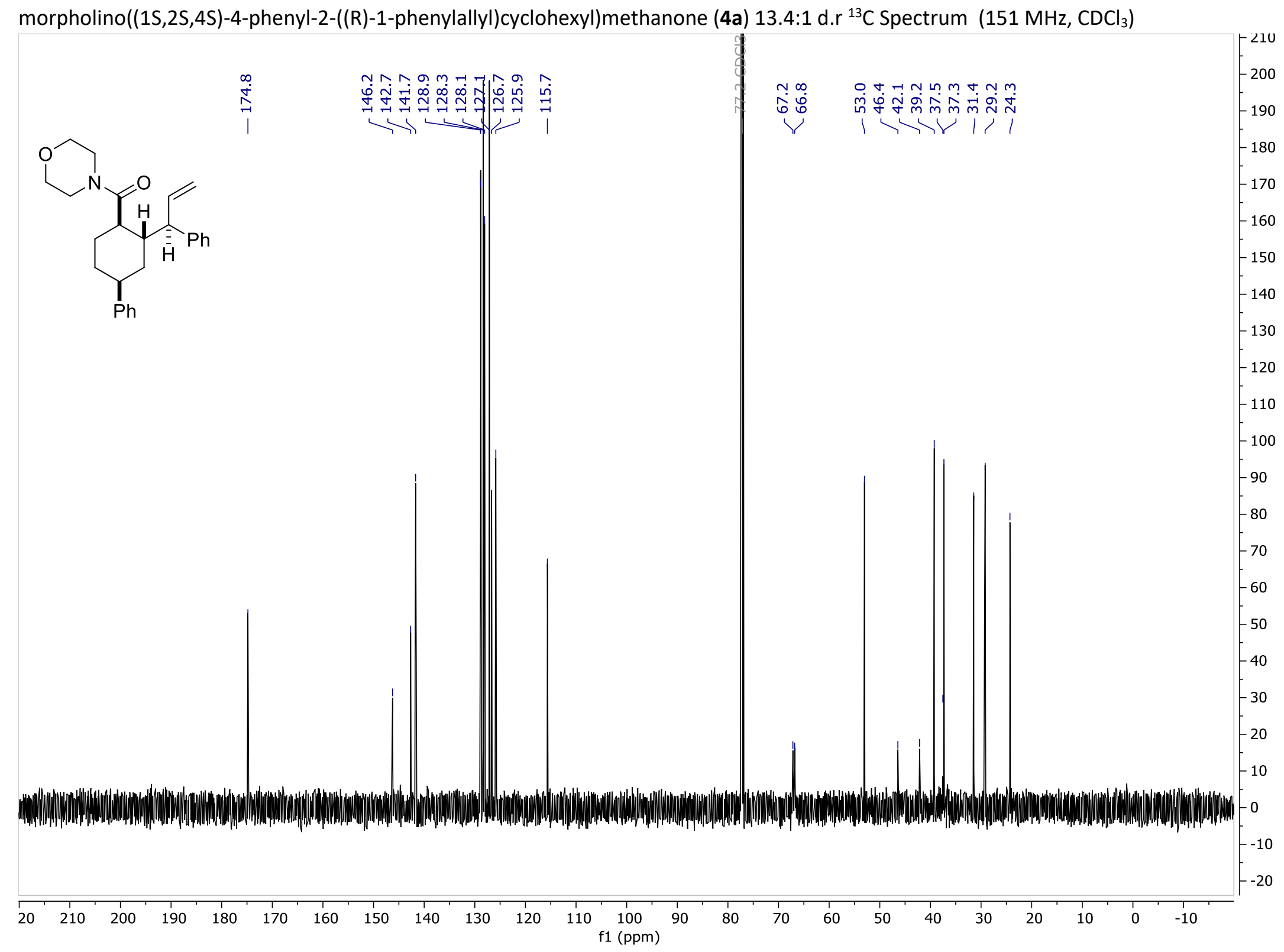


$(1 \mathrm{~S}, 2 \mathrm{~S}, 4 \mathrm{~S})-\mathrm{N}$-methoxy-N-methyl-4-phenyl-2-((R)-1-phenylallyl)cyclohexane-1-carboxamide (4b) >20:1 d.r. ${ }^{1} \mathrm{H}$ Spectrum $(600 \mathrm{MHz}, \mathrm{CDCl} 3)$

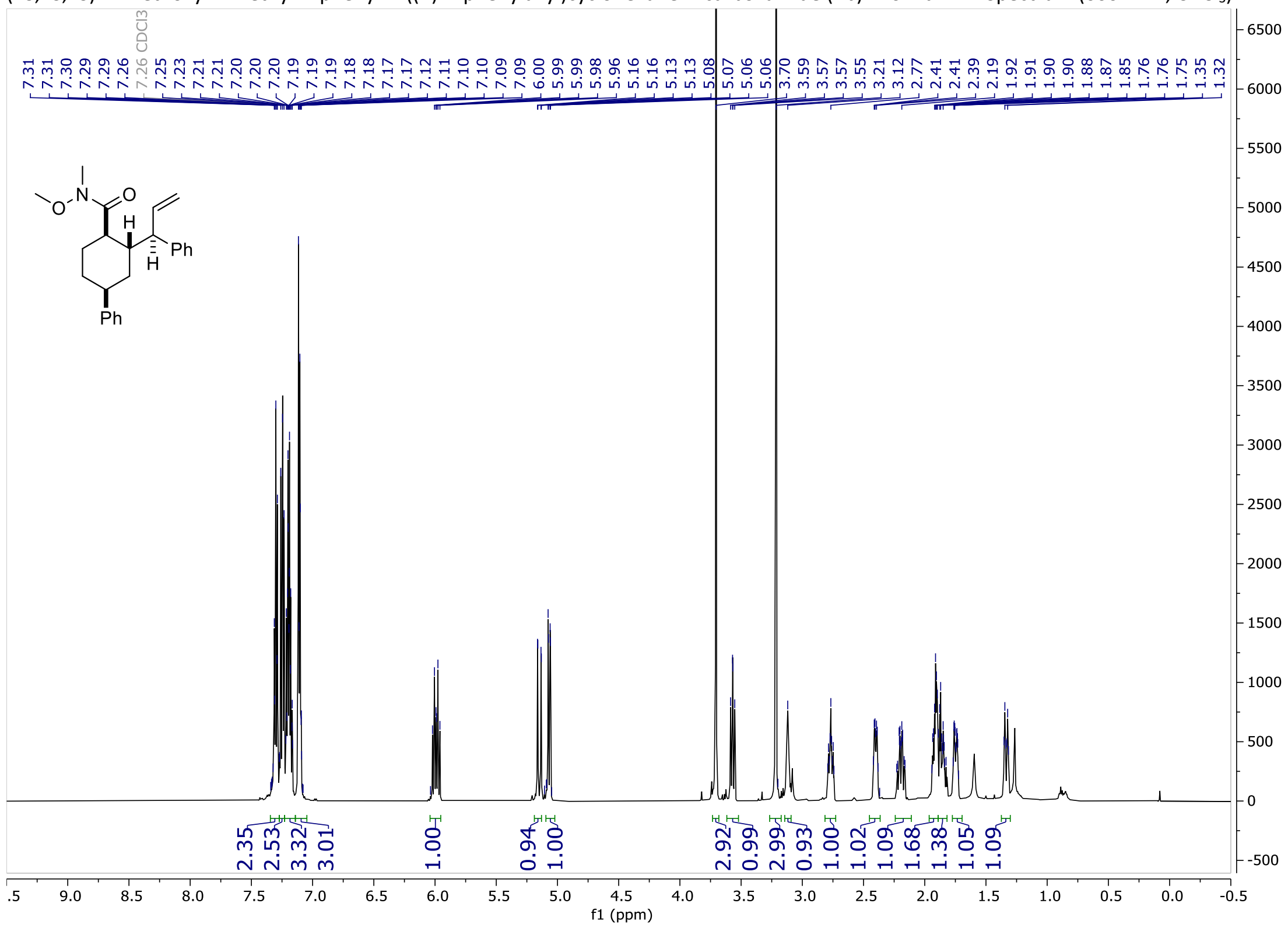


$(1 \mathrm{~S}, 2 \mathrm{~S}, 4 \mathrm{~S})-\mathrm{N}$-methoxy-N-methyl-4-phenyl-2-((R)-1-phenylallyl)cyclohexane-1-carboxamide (4b) ${ }^{13} \mathrm{C}$ Spectrum $(151 \mathrm{MHz}, \mathrm{CDCl} 3)$

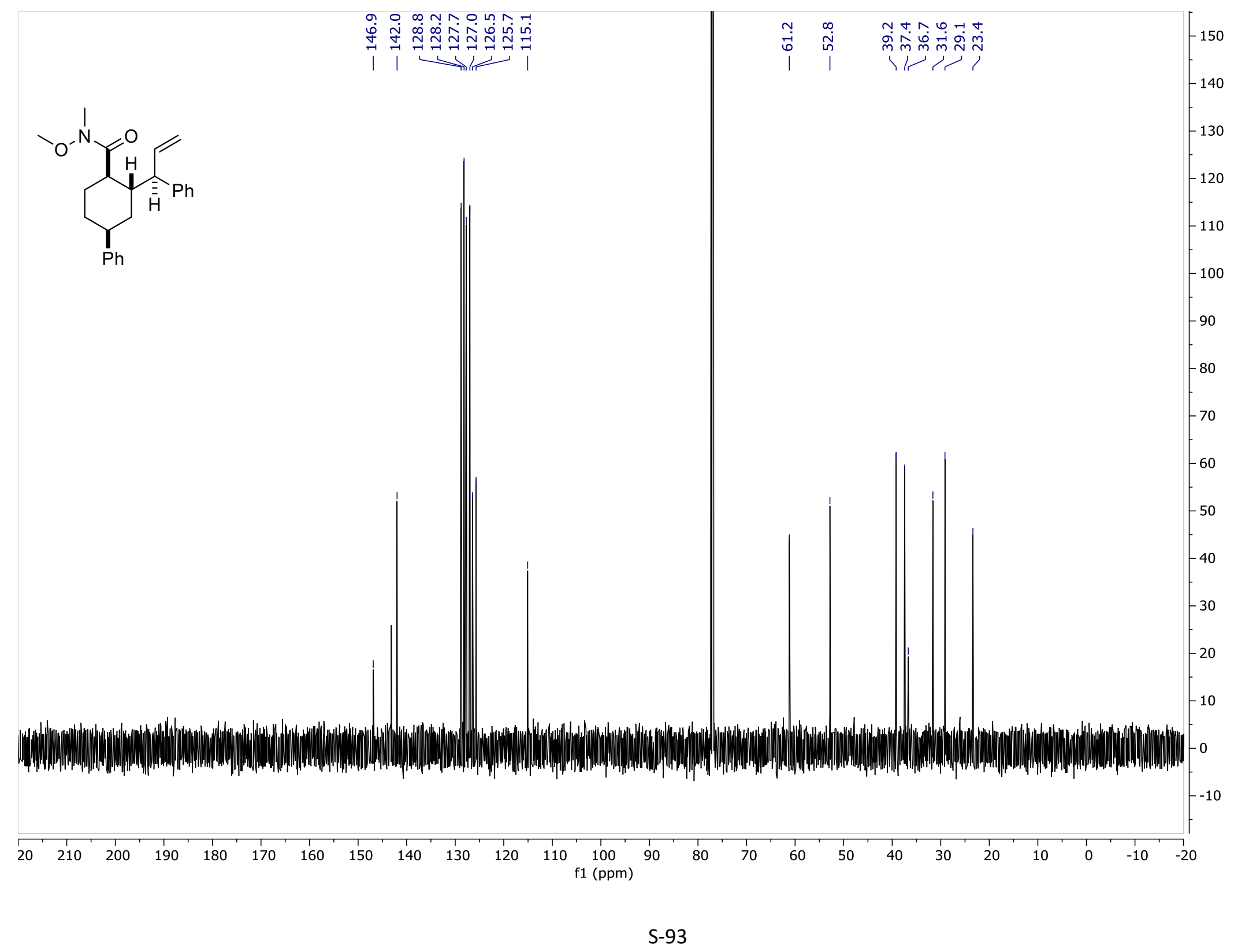


((1S,2S,4S)-4-methyl-2-((R)-1-phenylallyl)cyclohexyl)(morpholino)methanone (4c) 7:1 d.r. ${ }^{1} \mathrm{H}$ Spectrum $\left(600 \mathrm{MHz}, \mathrm{CDCl}_{3}\right)$

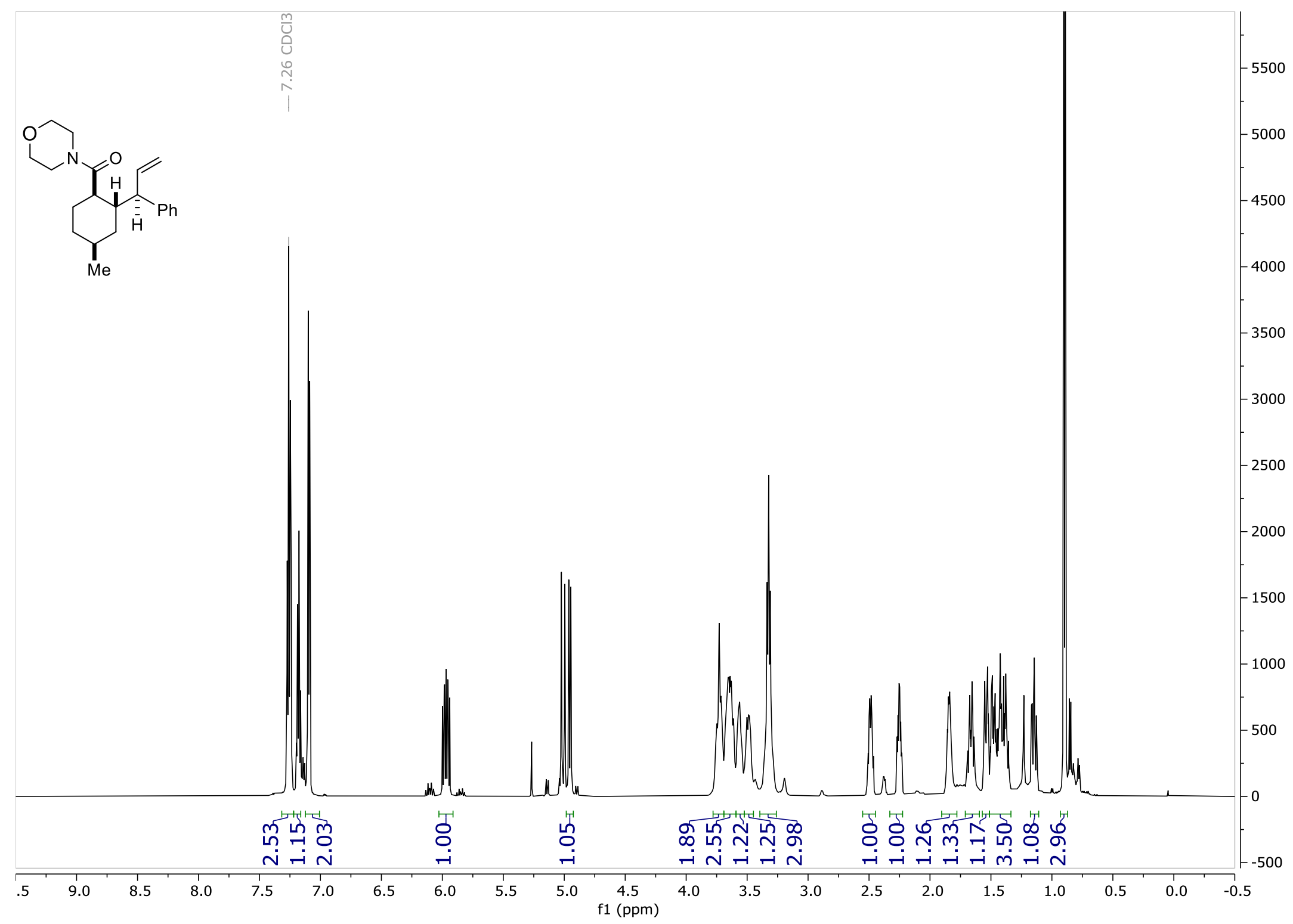


((1S,2S,4S)-4-methyl-2-((R)-1-phenylallyl)cyclohexyl)(morpholino)methanone $(4 \mathrm{c}){ }^{13} \mathrm{C}$ Spectrum $\left(151 \mathrm{MHz}, \mathrm{CDCl}_{3}\right)$

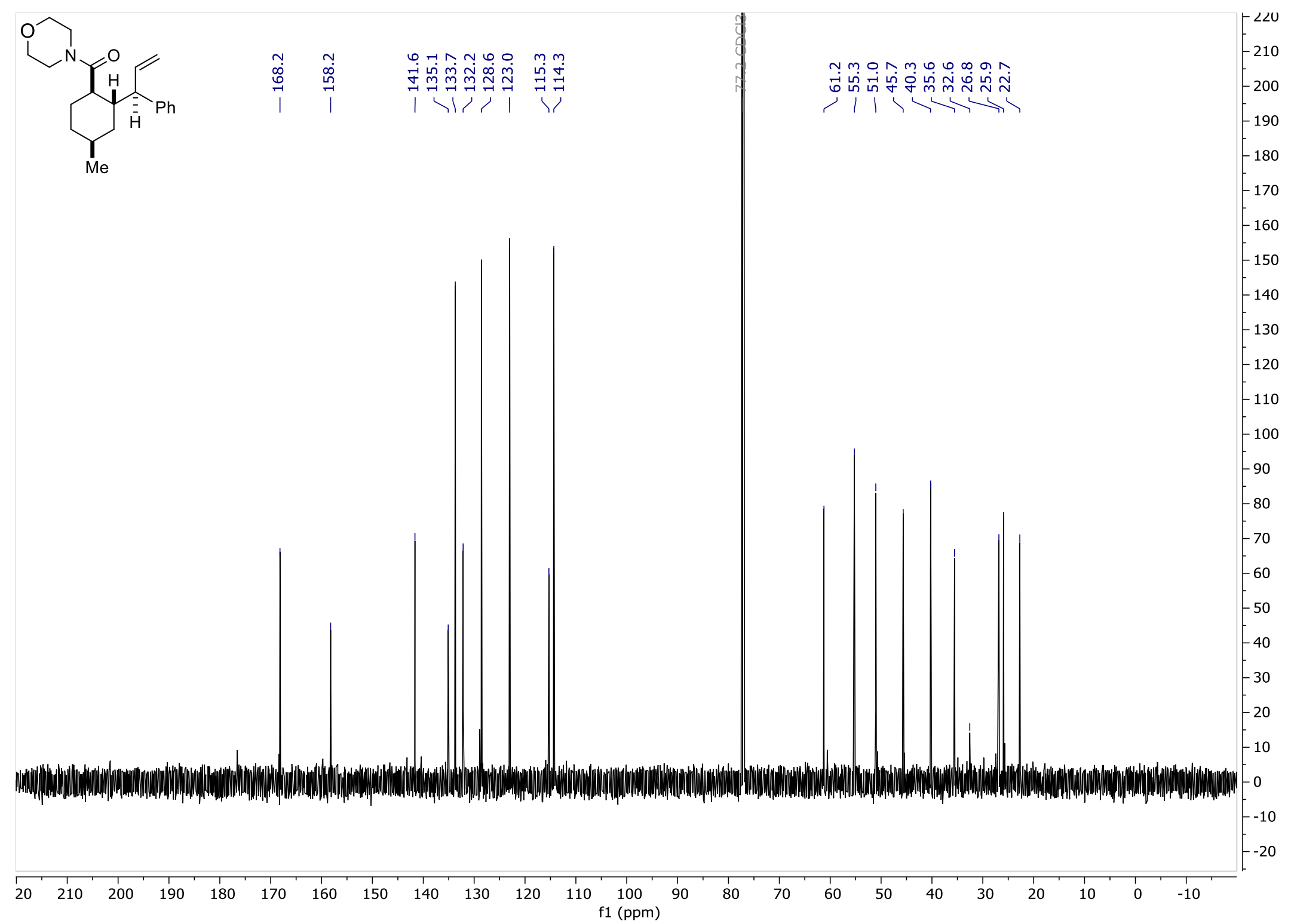


(1S,2S,4S)-N-methoxy-N,4-dimethyl-2-((R)-1-phenylallyl)cyclohexane-1-carboxamide (4d) 16.7:1 d.r. ${ }^{1} \mathrm{H}$ Spectrum (600 MHz, $\left.\mathrm{CDCl}_{3}\right)$

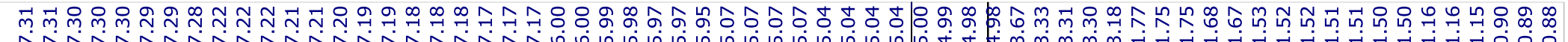

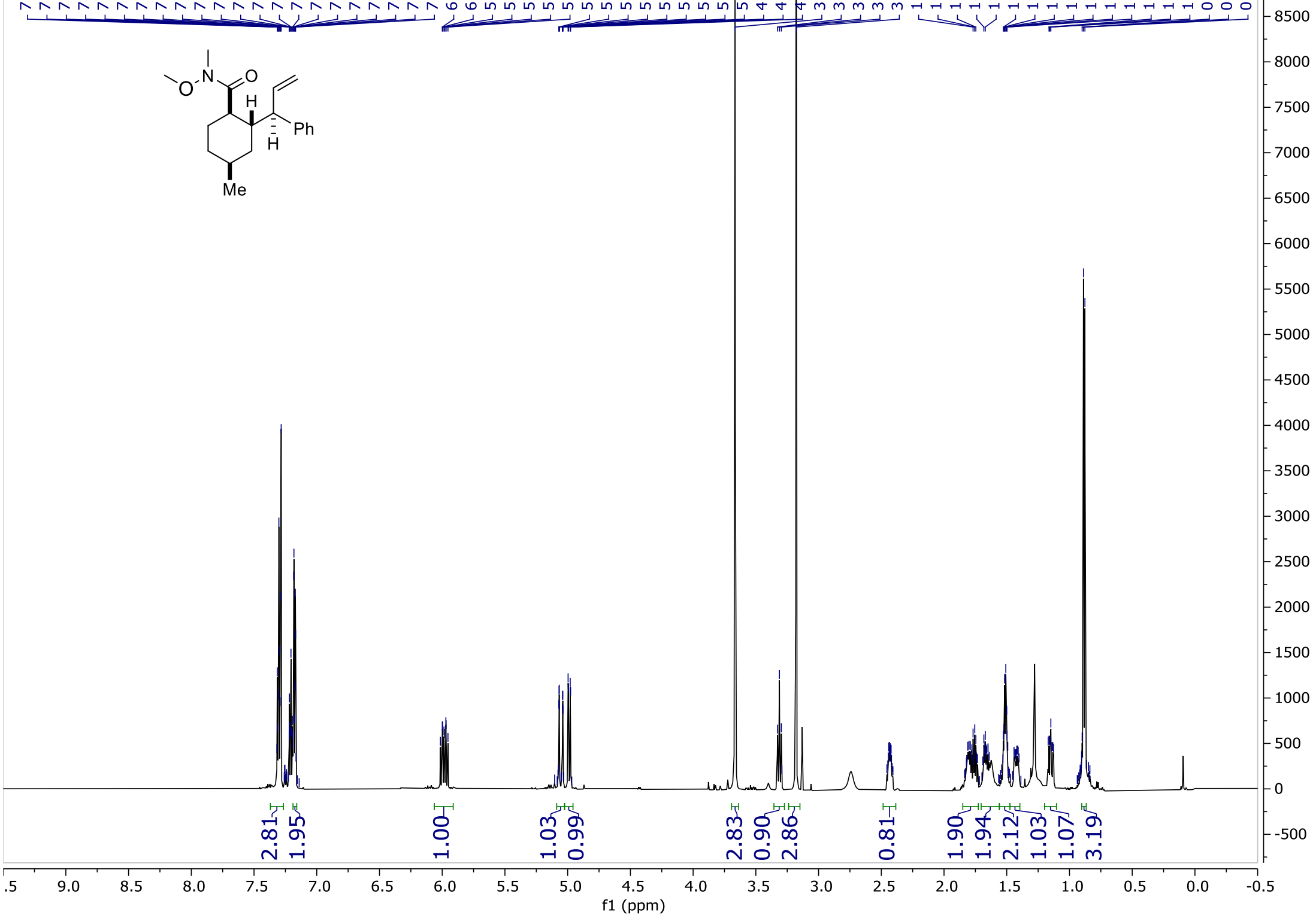


(1S,2S,4S)-N-methoxy-N,4-dimethyl-2-((R)-1-phenylallyl)cyclohexane-1-carboxamide (4d) 16.7:1 d.r. $\left.{ }^{13} \mathrm{C} \mathrm{Spectrum} \mathrm{(151} \mathrm{MHz,} \mathrm{CDCl} 3\right)$

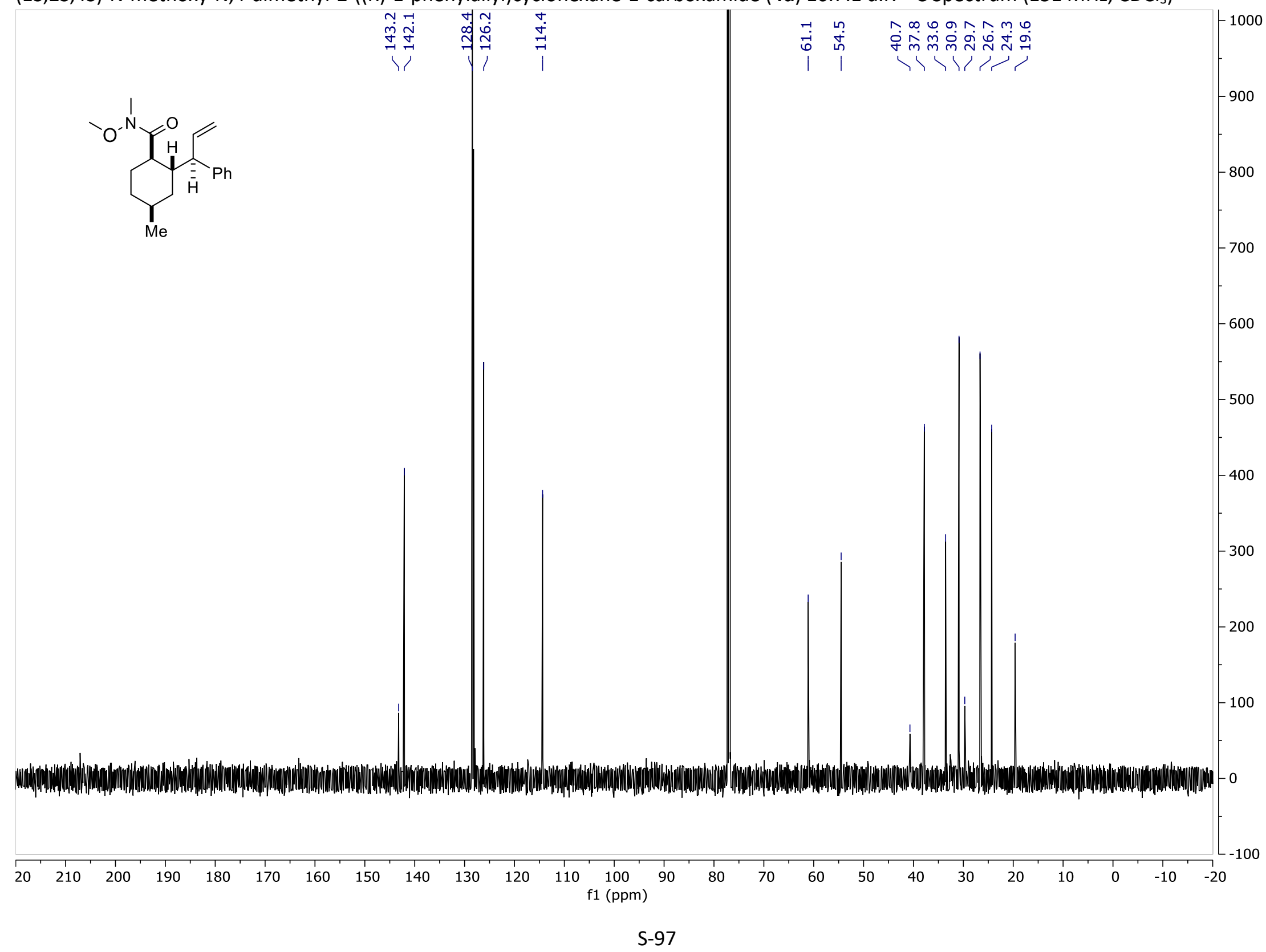


((1S,2S,4S)-4-isopropyl-2-((R)-1-phenylallyl)cyclohexyl)(morpholino)methanone (4e) 11.4:1 d.r. ${ }^{1} \mathrm{H}$ Spectrum $\left(600 \mathrm{MHz}, \mathrm{CDCl}_{3}\right)$

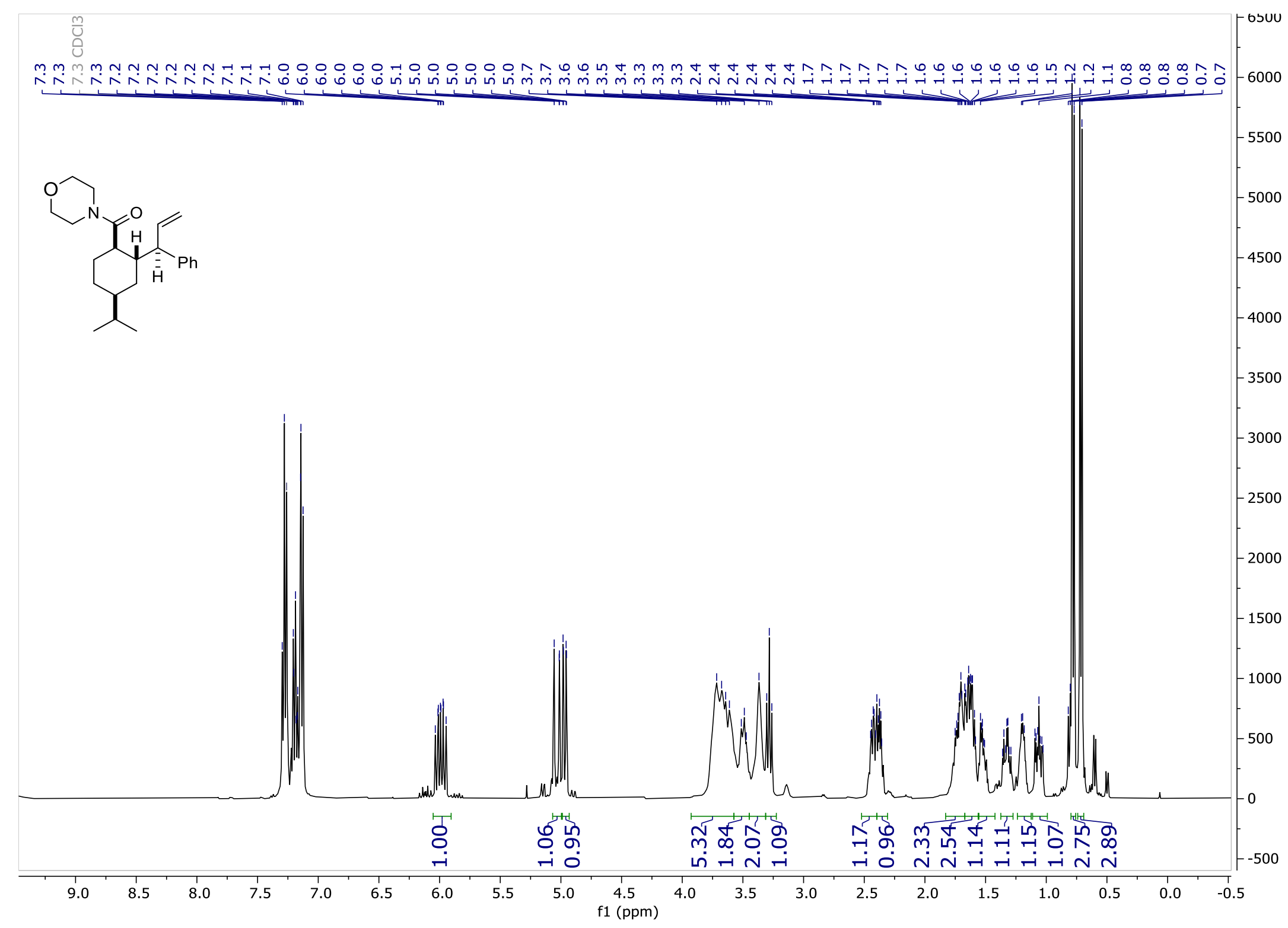


((1S,2S,4S)-4-isopropyl-2-((R)-1-phenylallyl)cyclohexyl)(morpholino)methanone (4e) 11.4:1 d.r. ${ }^{13} \mathrm{C}$ Spectrum (151 MHz, $\left.\mathrm{CDCl}_{3}\right)$

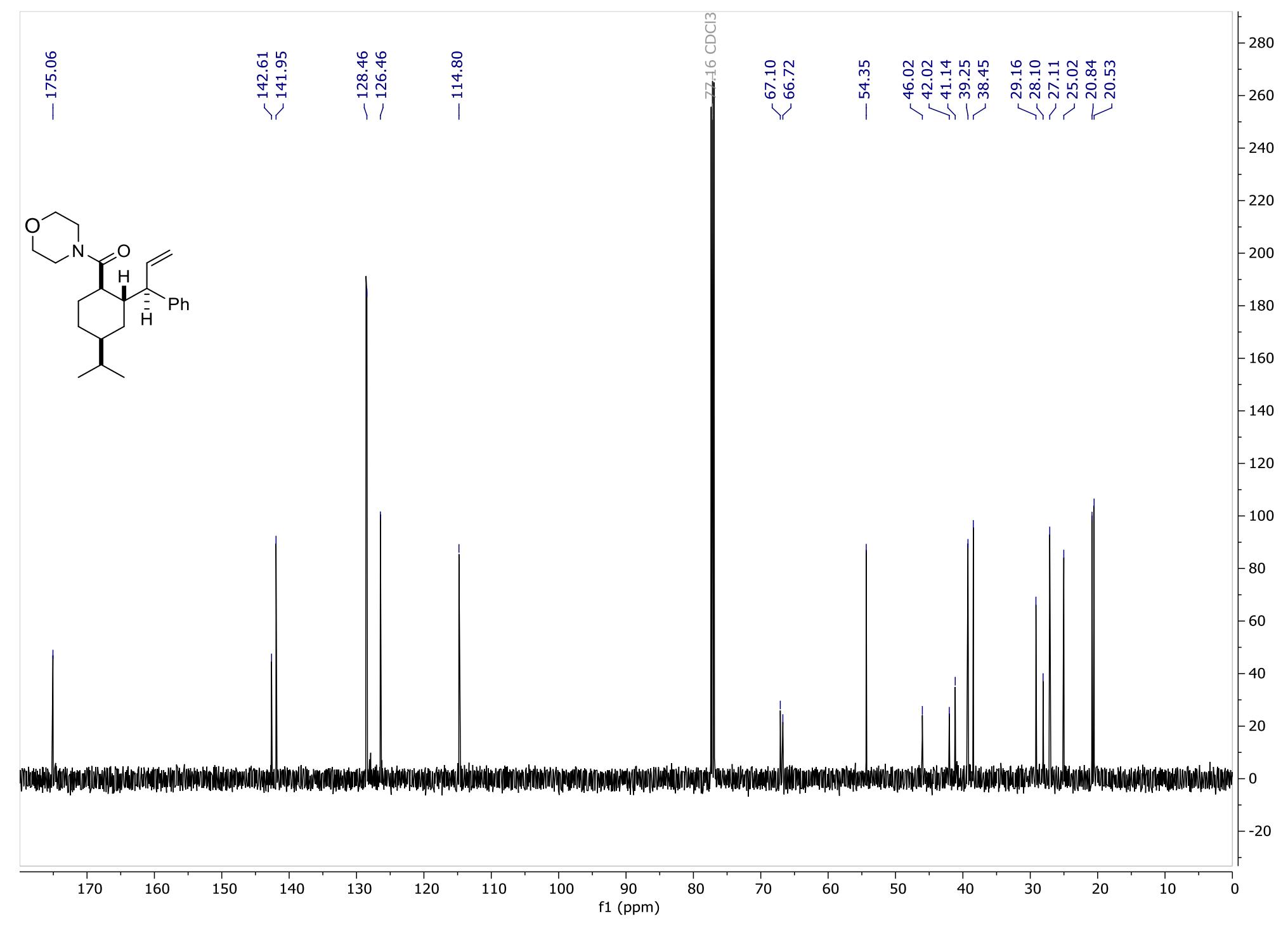


ethyl (1S,3S,4S)-4-(morpholine-4-carbonyl)-3-((R)-1-phenylallyl)cyclohexane-1-carboxylate (4f) 8.3:1 d.r. ${ }^{1} \mathrm{H}$ Spectrum $\left(600 \mathrm{MHz}^{\mathrm{CDCl}}{ }_{3}\right)$

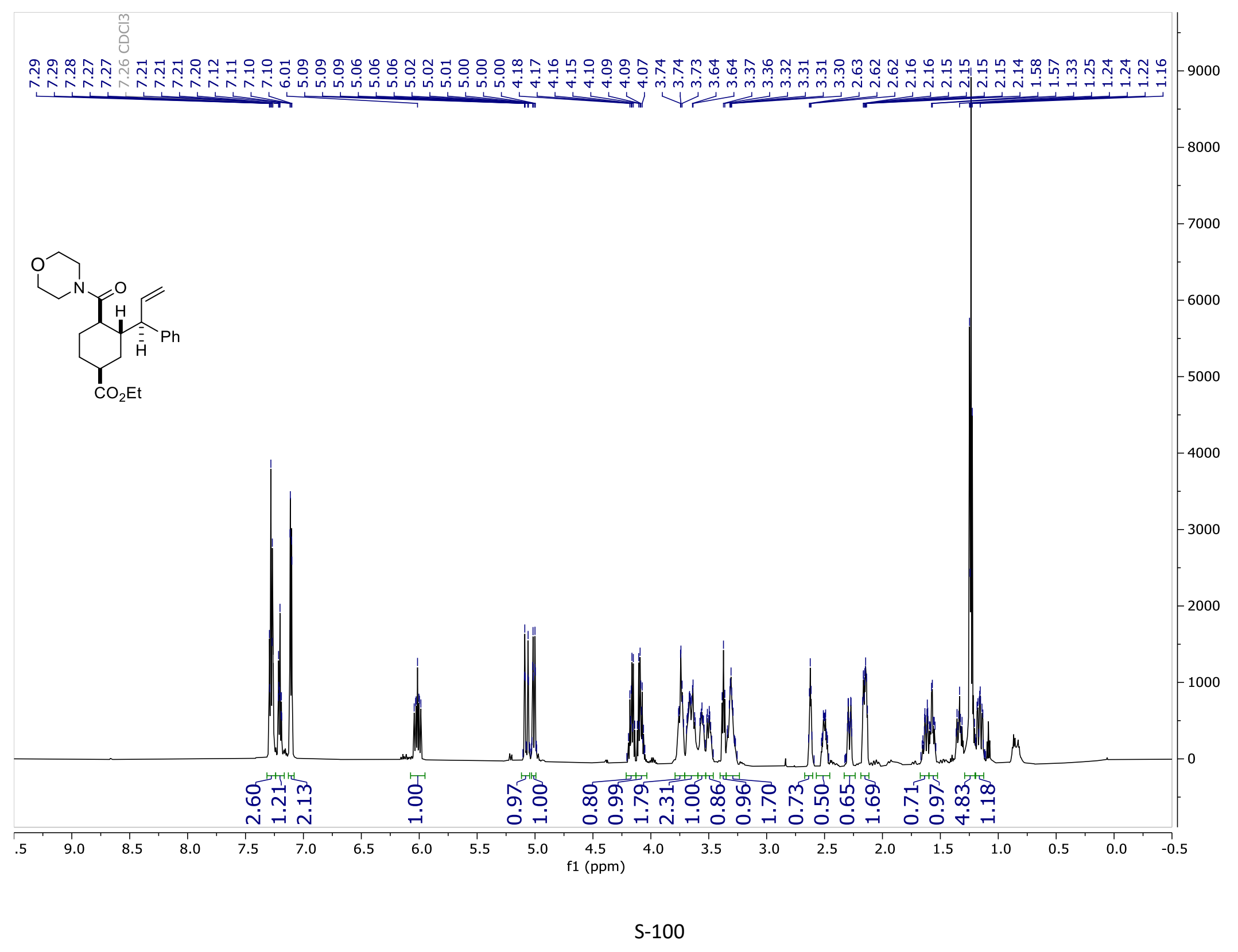


ethyl (1S,3S,4S)-4-(morpholine-4-carbonyl)-3-((R)-1-phenylallyl)cyclohexane-1-carboxylate (4f) ${ }^{13} \mathrm{C}$ Spectrum (151 $\mathrm{MHz}^{\mathrm{CDCl}} \mathrm{CD}_{3}$

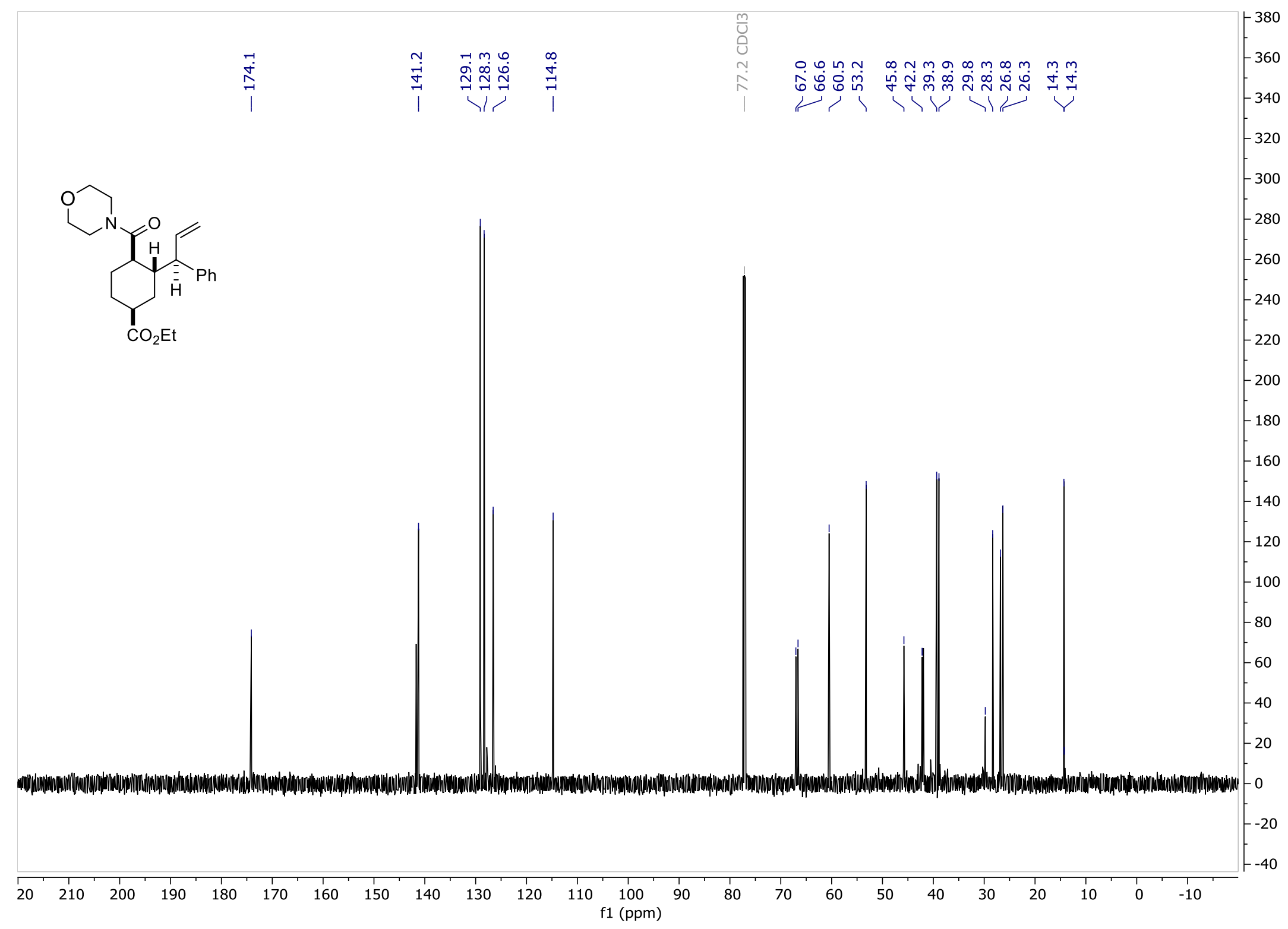


Ethyl (1S,3S,4S)-4-(methoxy(methyl)carbamoyl)-3-((R)-1-phenylallyl)cyclohexane-1-carboxylate (4g) >20:1 d.r. ${ }^{1} \mathrm{H} \mathrm{Spectrum}\left(600 \mathrm{MHz}, \mathrm{CDCl}_{3}\right)$

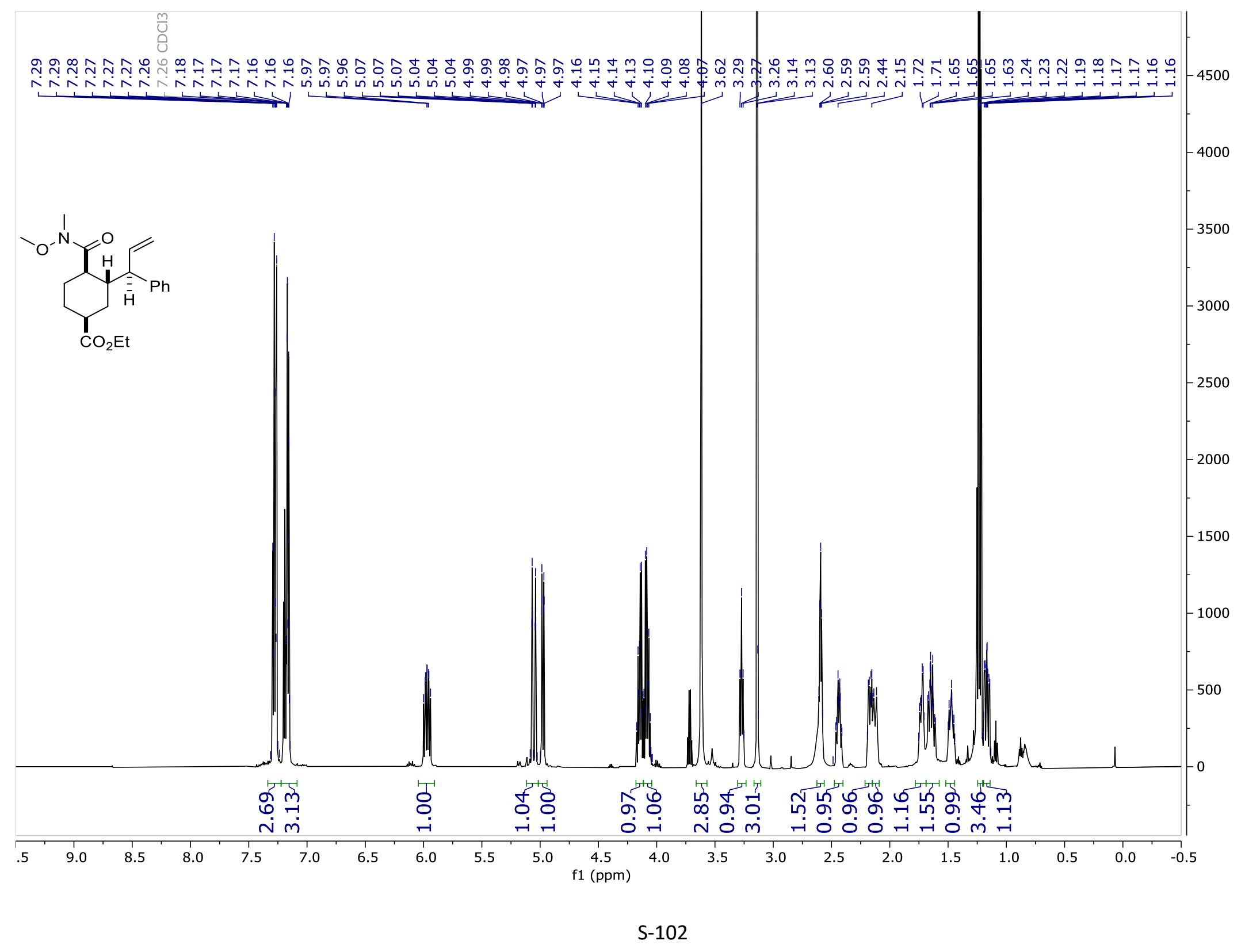


Ethyl (1S,3S,4S)-4-(methoxy(methyl)carbamoyl)-3-((R)-1-phenylallyl)cyclohexane-1-carboxylate $(\mathbf{4 g}){ }^{13} \mathrm{C} \mathrm{Spectrum}\left(151 \mathrm{MHz}, \mathrm{CDCl}_{3}\right)$

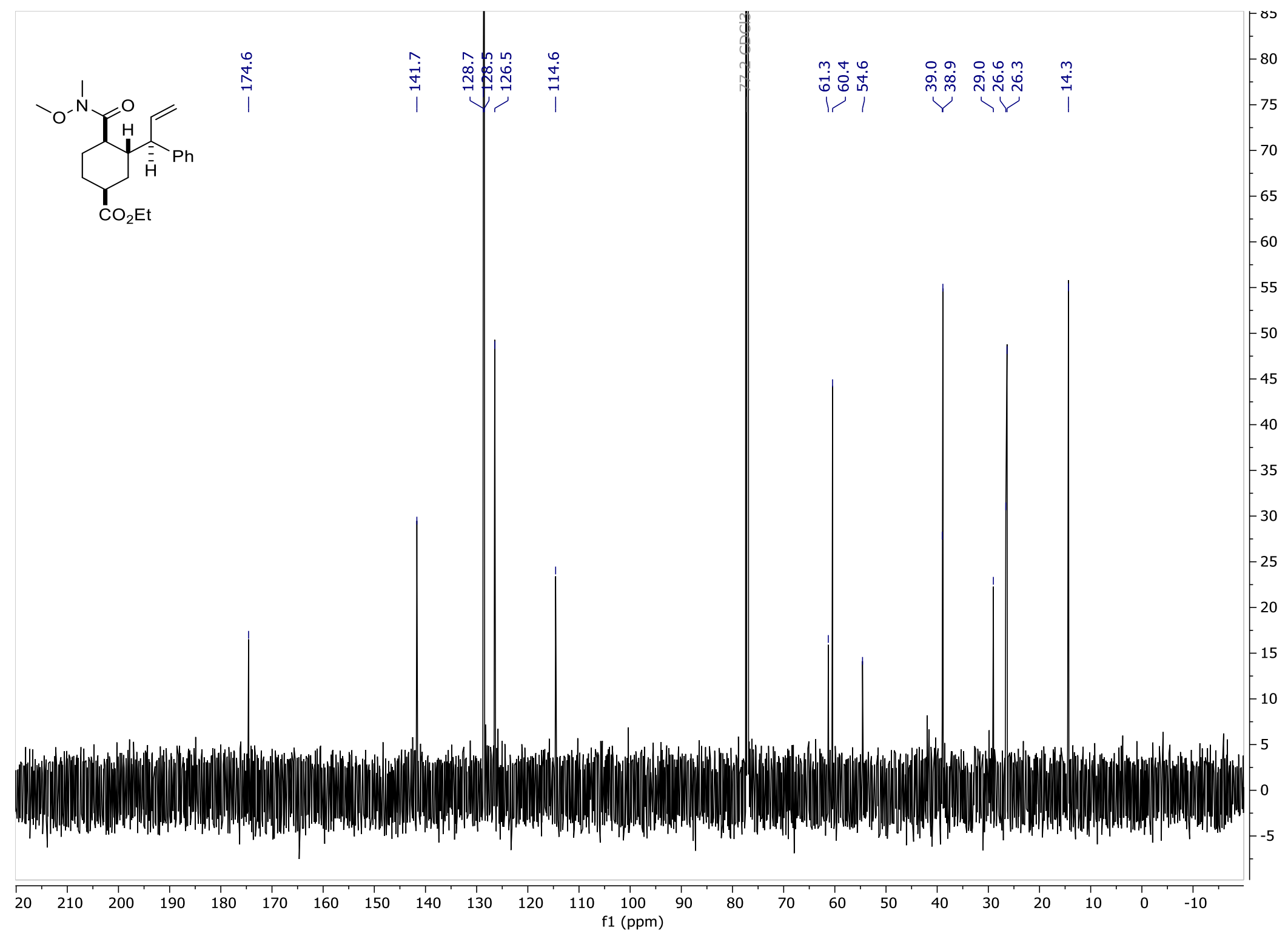

S-103 
$\mathrm{N}-\left((1 \mathrm{~S}, 3 \mathrm{~S}, 4 \mathrm{~S})-4-\left(\right.\right.$ morpholine-4-carbonyl)-3-((R)-1-phenylallyl)cyclohexyl)acetamide (4h) 3.4:1 d.r. ${ }^{1} \mathrm{H}$ Spectrum $\left(600 \mathrm{MHz}, \mathrm{CDCl}_{3}\right)$

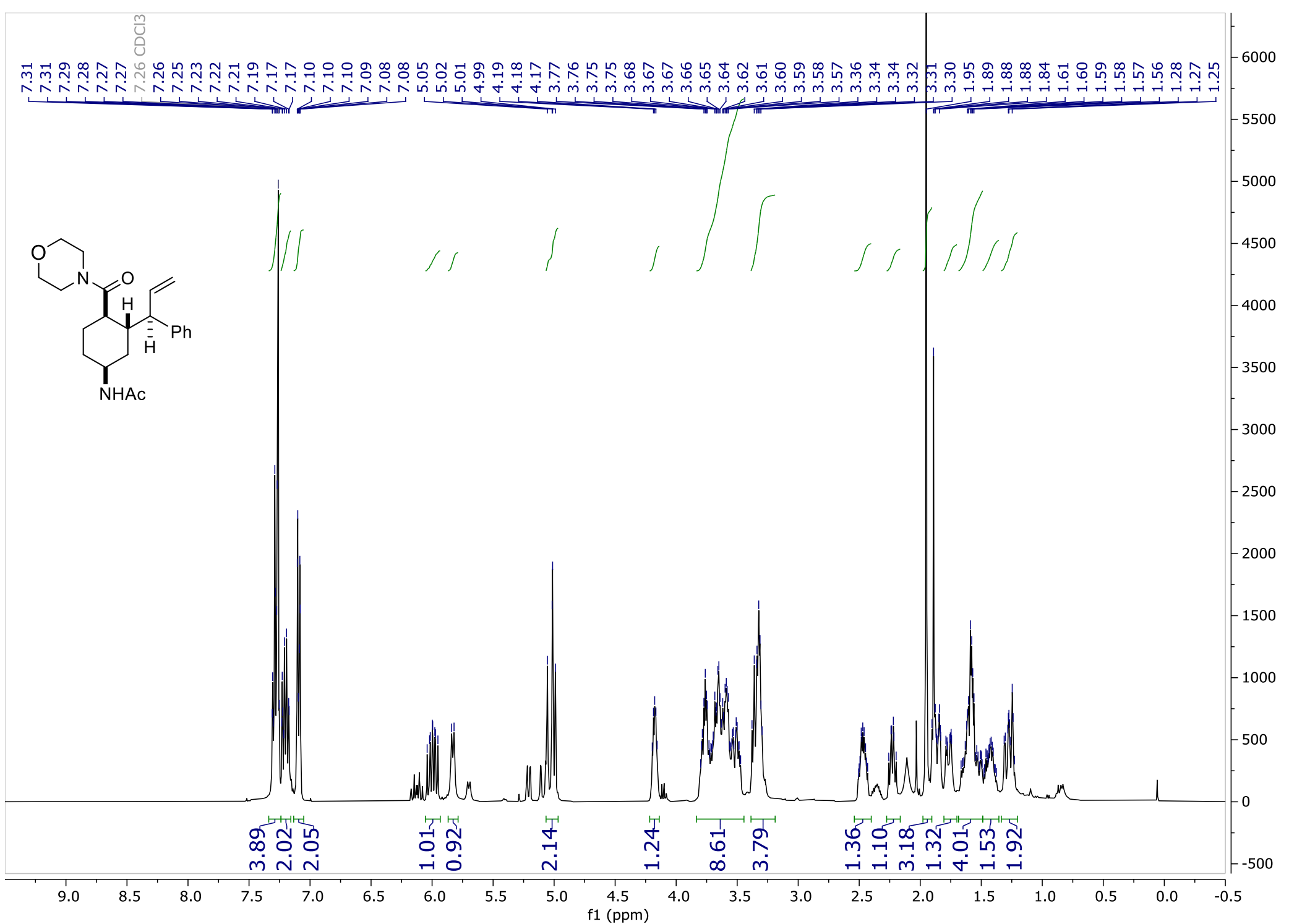


$\mathrm{N}-\left((1 \mathrm{~S}, 3 \mathrm{~S}, 4 \mathrm{~S})-4-\left(\right.\right.$ morpholine-4-carbonyl)-3-((R)-1-phenylallyl)cyclohexyl)acetamide (4h) ${ }^{13} \mathrm{C}$ Spectrum (151 MHz, $\left.\mathrm{CDCl}_{3}\right)$

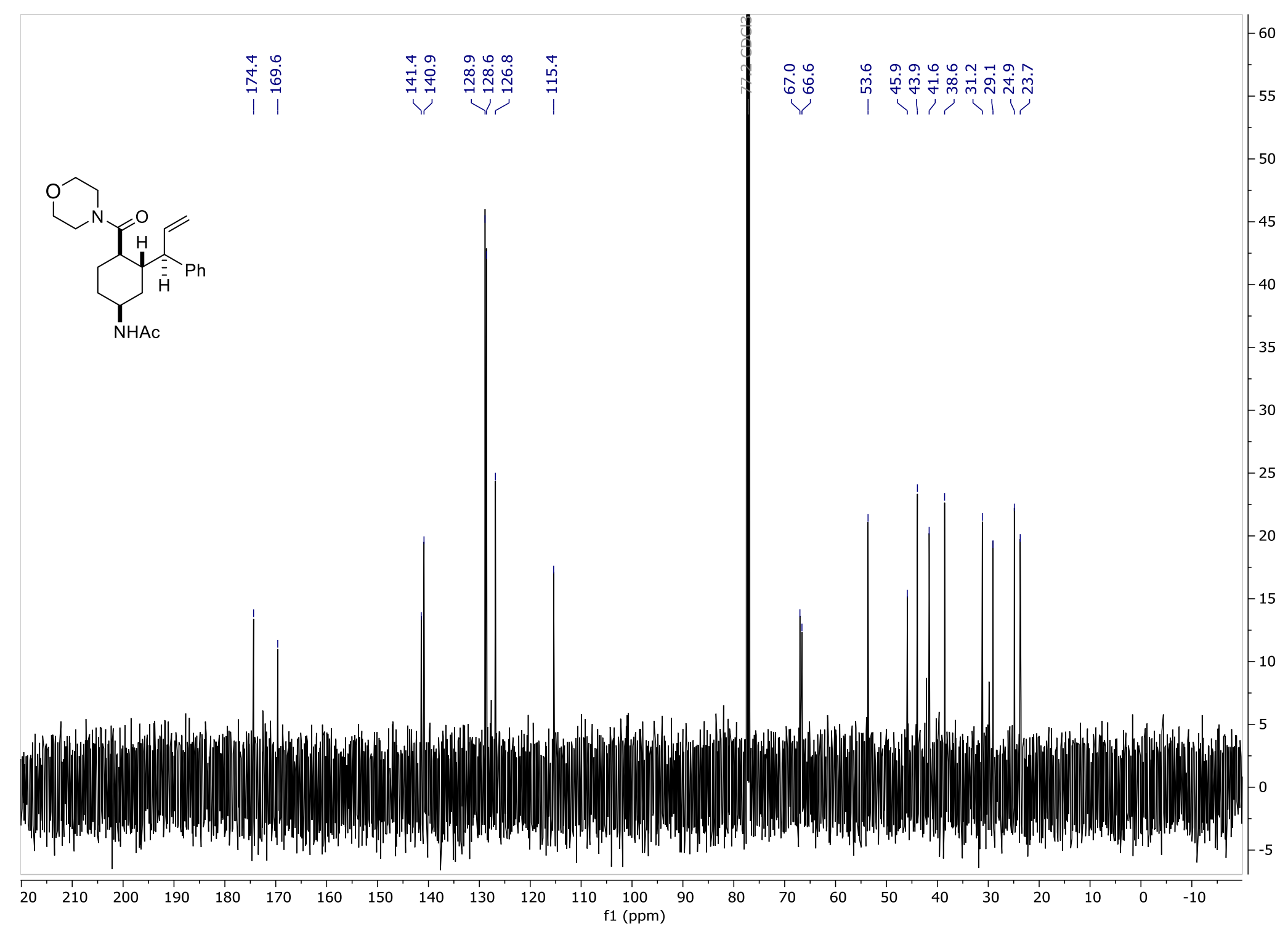


$(1 \mathrm{~S}, 2 \mathrm{~S}, 4 \mathrm{~S})-4$-acetamido-N-methoxy-N-methyl-2-((R)-1-phenylallyl)cyclohexane-1-carboxamide (4i) 19:1 d.r. ${ }^{1} \mathrm{H}$ Spectrum $\left(400 \mathrm{MHz}, \mathrm{CDCl}_{3}\right)$

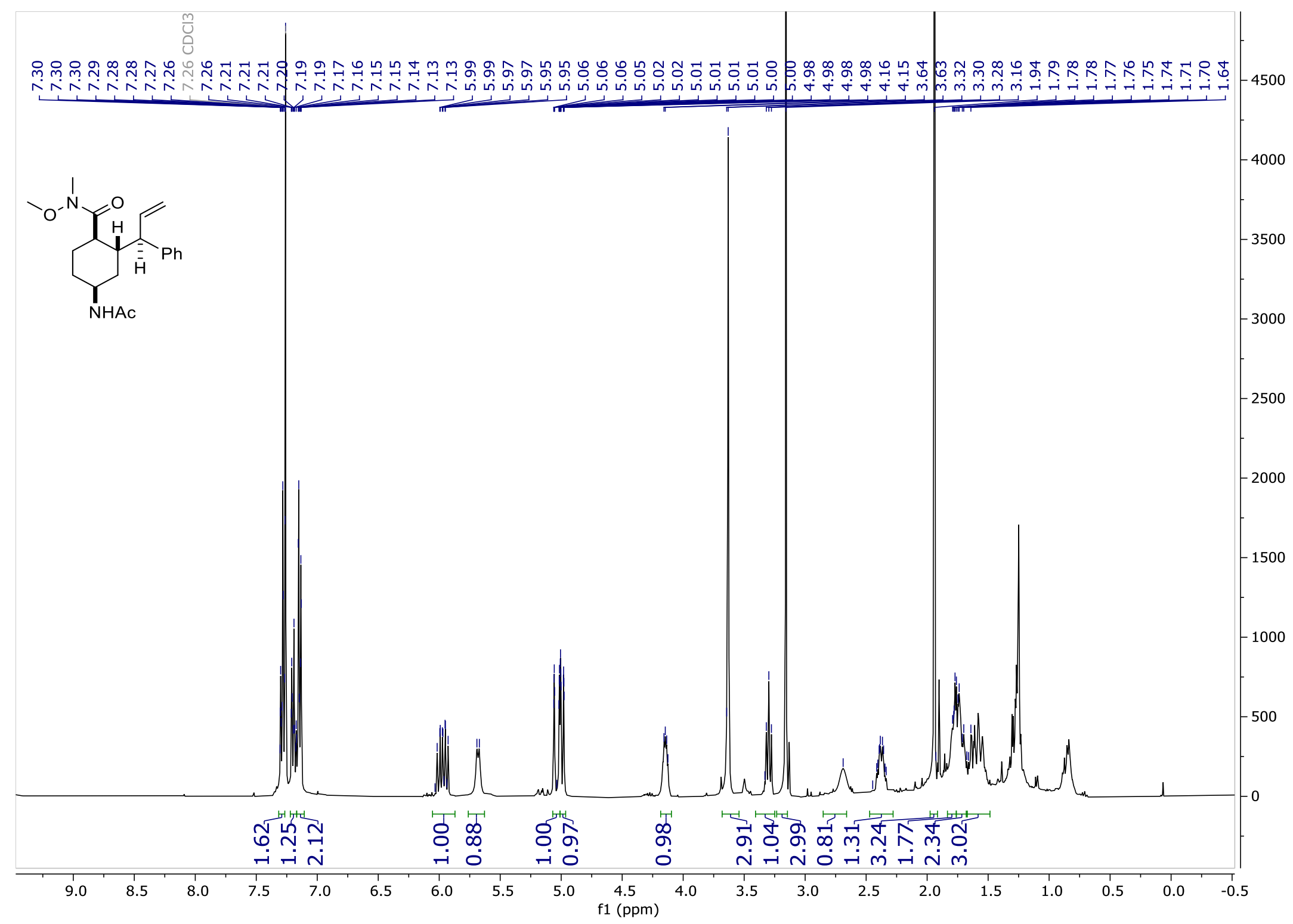

S-106 
$(1 \mathrm{~S}, 2 \mathrm{~S}, 4 \mathrm{~S})$-4-acetamido-N-methoxy-N-methyl-2-((R)-1-phenylallyl)cyclohexane-1-carboxamide $(4 \mathrm{i}){ }^{13} \mathrm{C}$ Spectrum $\left(151 \mathrm{MHz}, \mathrm{CDCl}_{3}\right)$

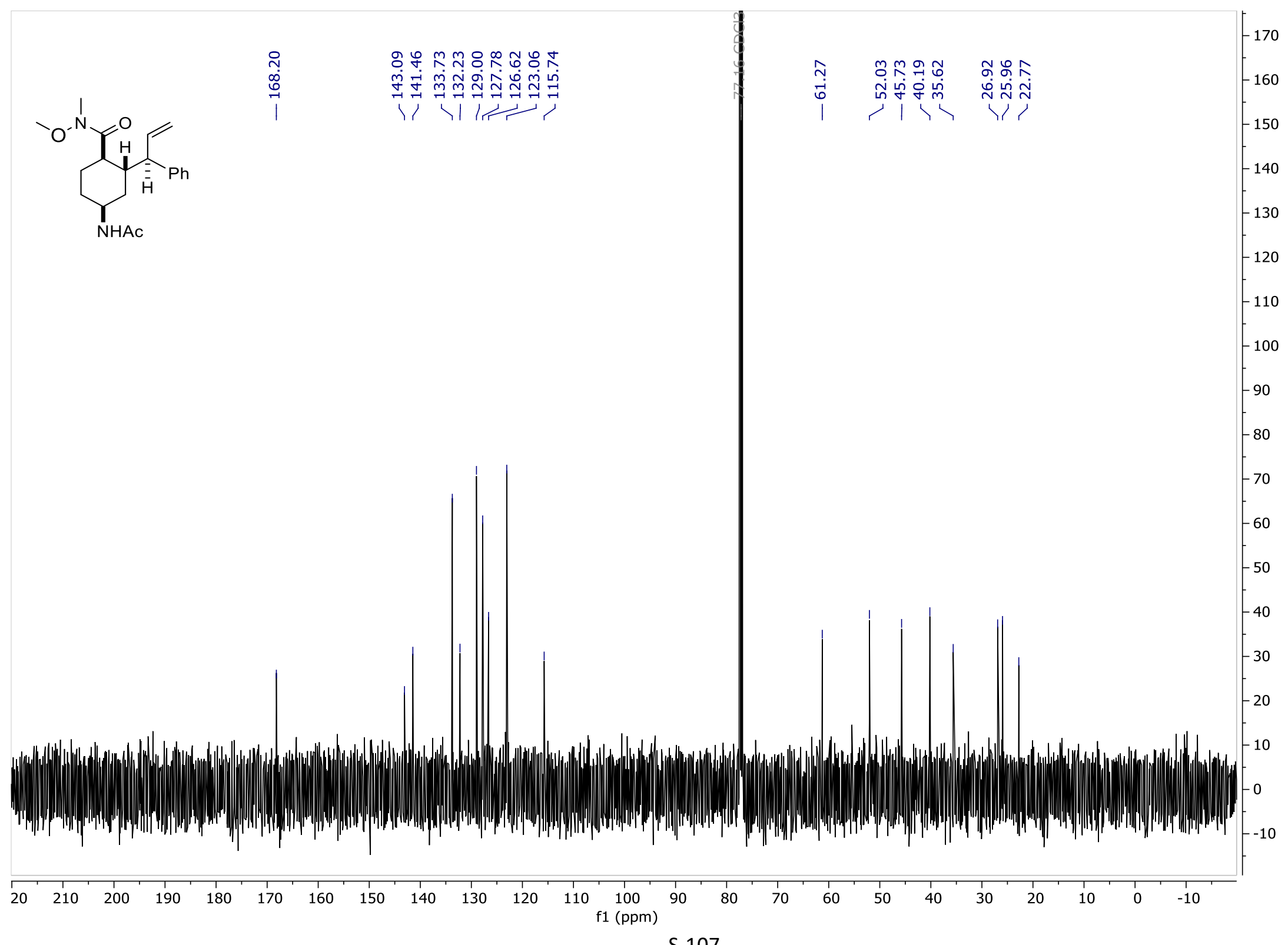

S-107 
2-((1S,3S,4S)-4-(morpholine-4-carbonyl)-3-((R)-1-phenylallyl)cyclohexyl)isoindoline-1,3-dione (4j) 10:1 d.r. ${ }^{1} \mathrm{H}$ Spectrum $(600 \mathrm{MHz}, \mathrm{CDCl} 3)$

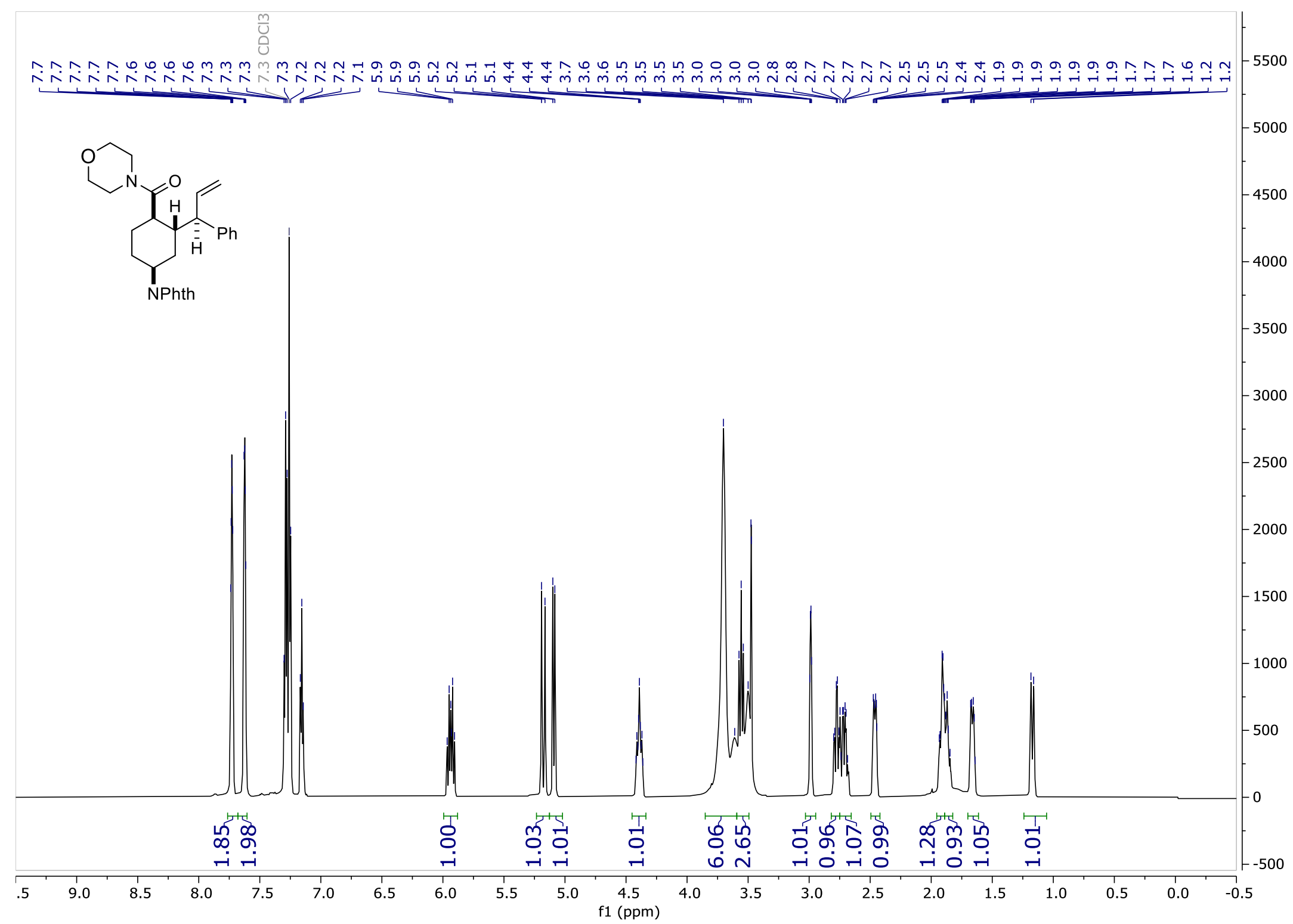

S-108 
2-((1S,3S,4S)-4-(morpholine-4-carbonyl)-3-((R)-1-phenylallyl)cyclohexyl)isoindoline-1,3-dione (4j) ${ }^{13} \mathrm{C}$ Spectrum $\left(151 \mathrm{MHz}, \mathrm{CDCl}_{3}\right)$

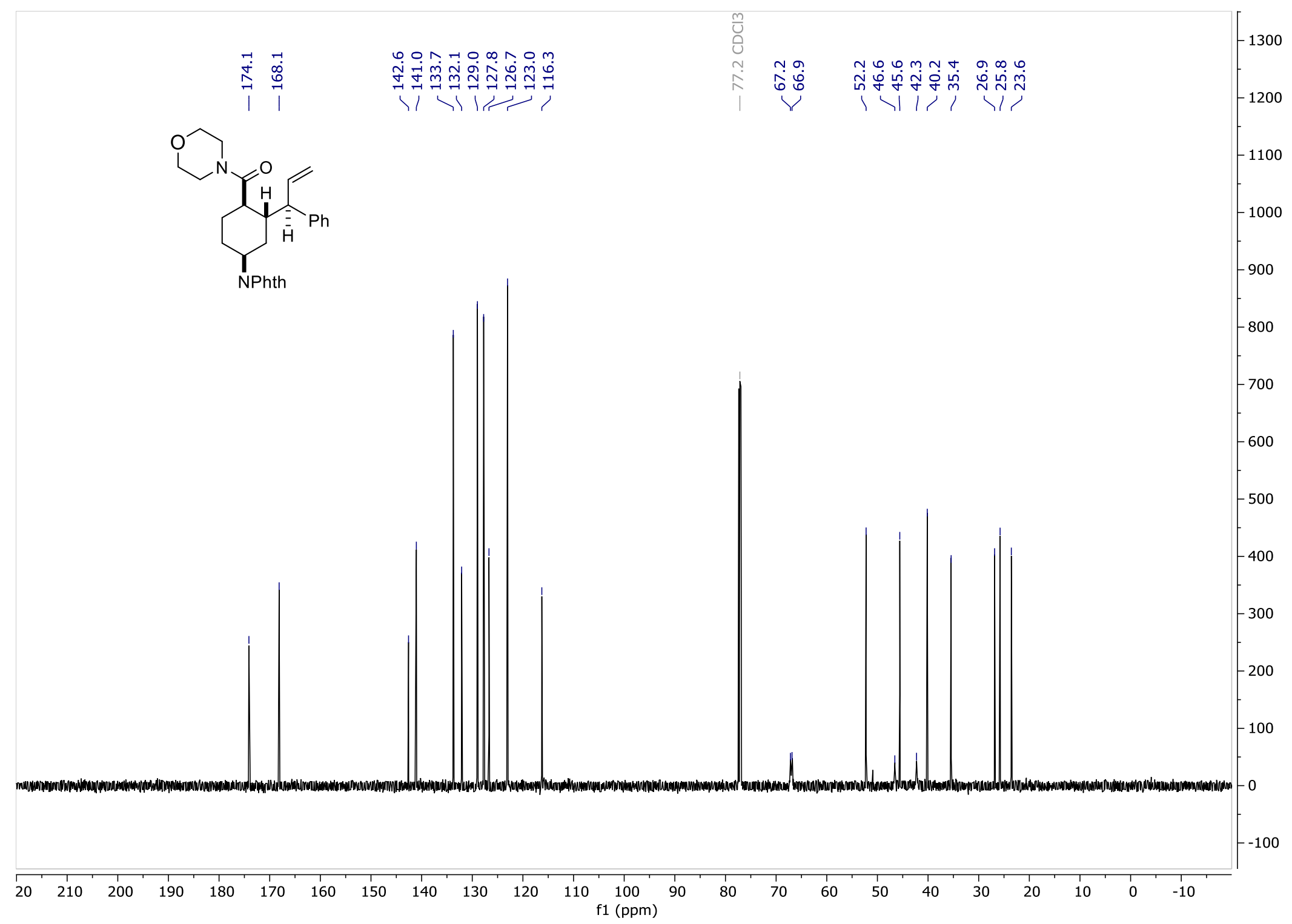


2-((1S,3S,4S)-4-(morpholine-4-carbonyl)-3-((R)-1-phenylallyl)cyclohexyl)isoindoline-1,3-dione (4j) ${ }^{1} \mathrm{H}$ Spectrum from 11.9:1 d.r. (3f) $(600 \mathrm{MHz}$ $\left.\mathrm{CDCl}_{3}\right)$

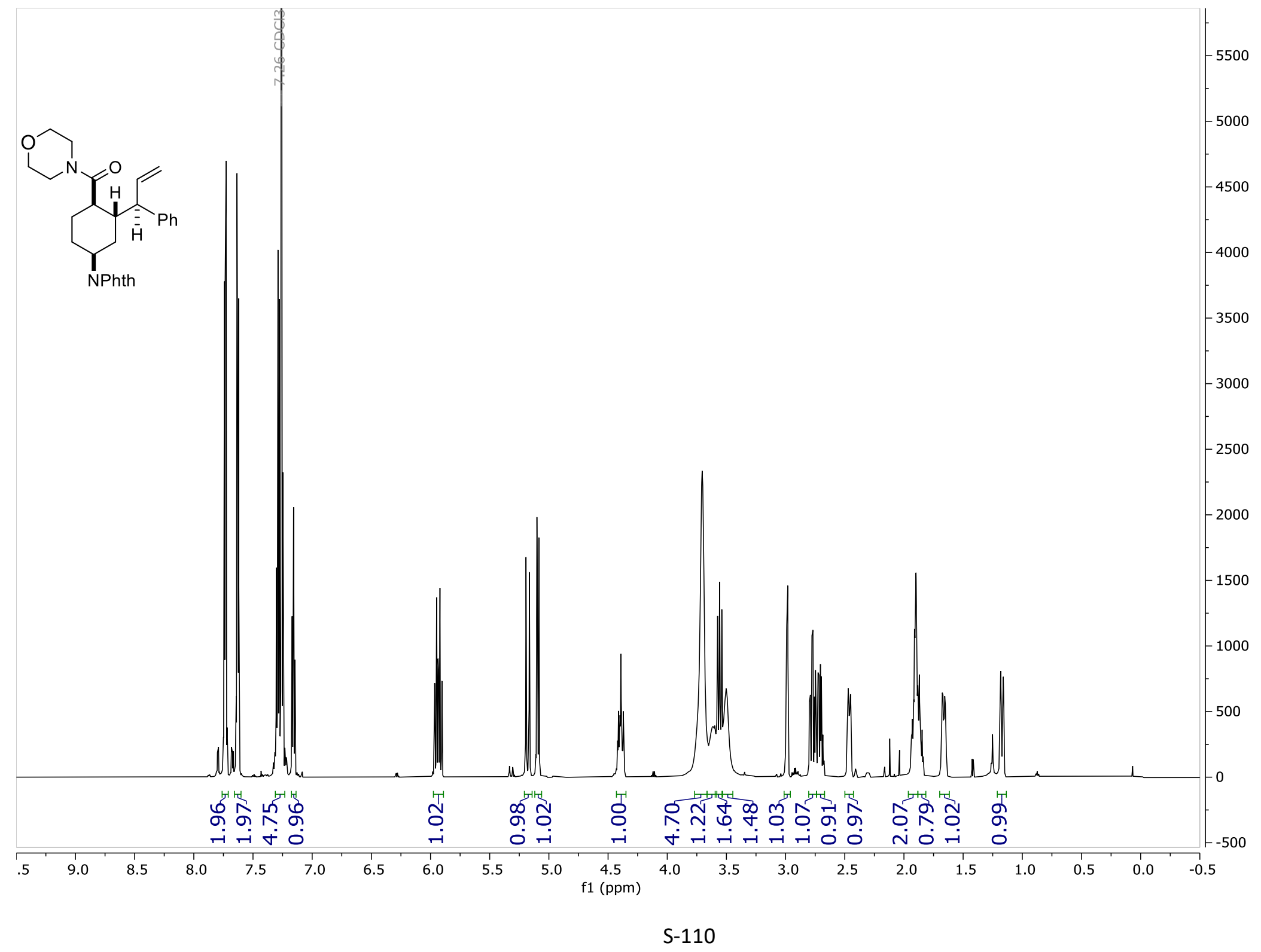


(1S,2S,4S)-4-(1,3-dioxoisoindolin-2-yl)-N-methoxy-N-methyl-2-((R)-1-phenylallyl)cyclohexane-1-carboxamide (4k) 9.1:1 d.r. (600 MHz, CDCl $)$

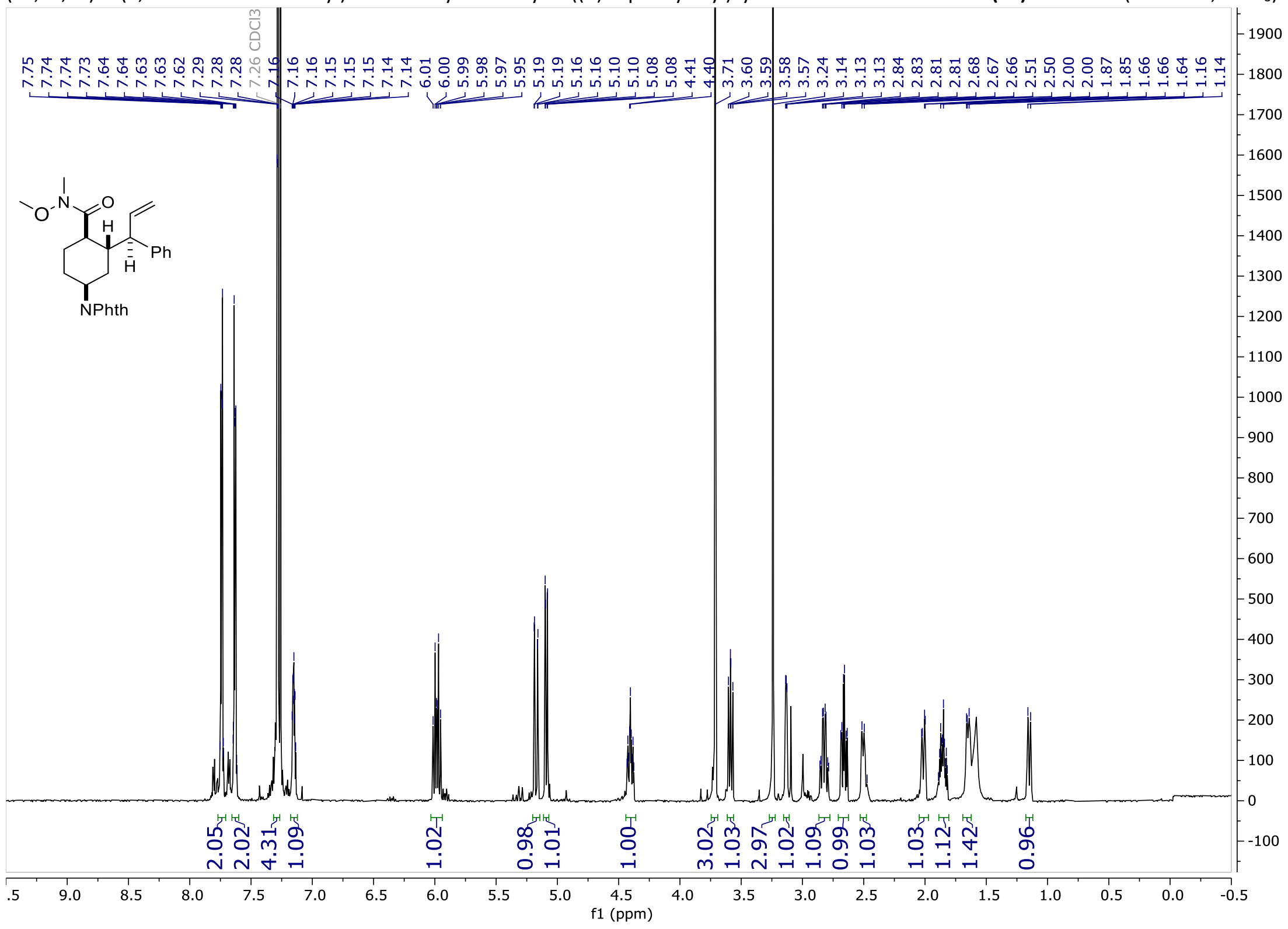

S-111 
(1S,2S,4S)-4-(1,3-dioxoisoindolin-2-yl)-N-methoxy-N-methyl-2-((R)-1-phenylallyl)cyclohexane-1-carboxamide (4k) (151 MHz, $\left.\mathrm{CDCl}_{3}\right)$

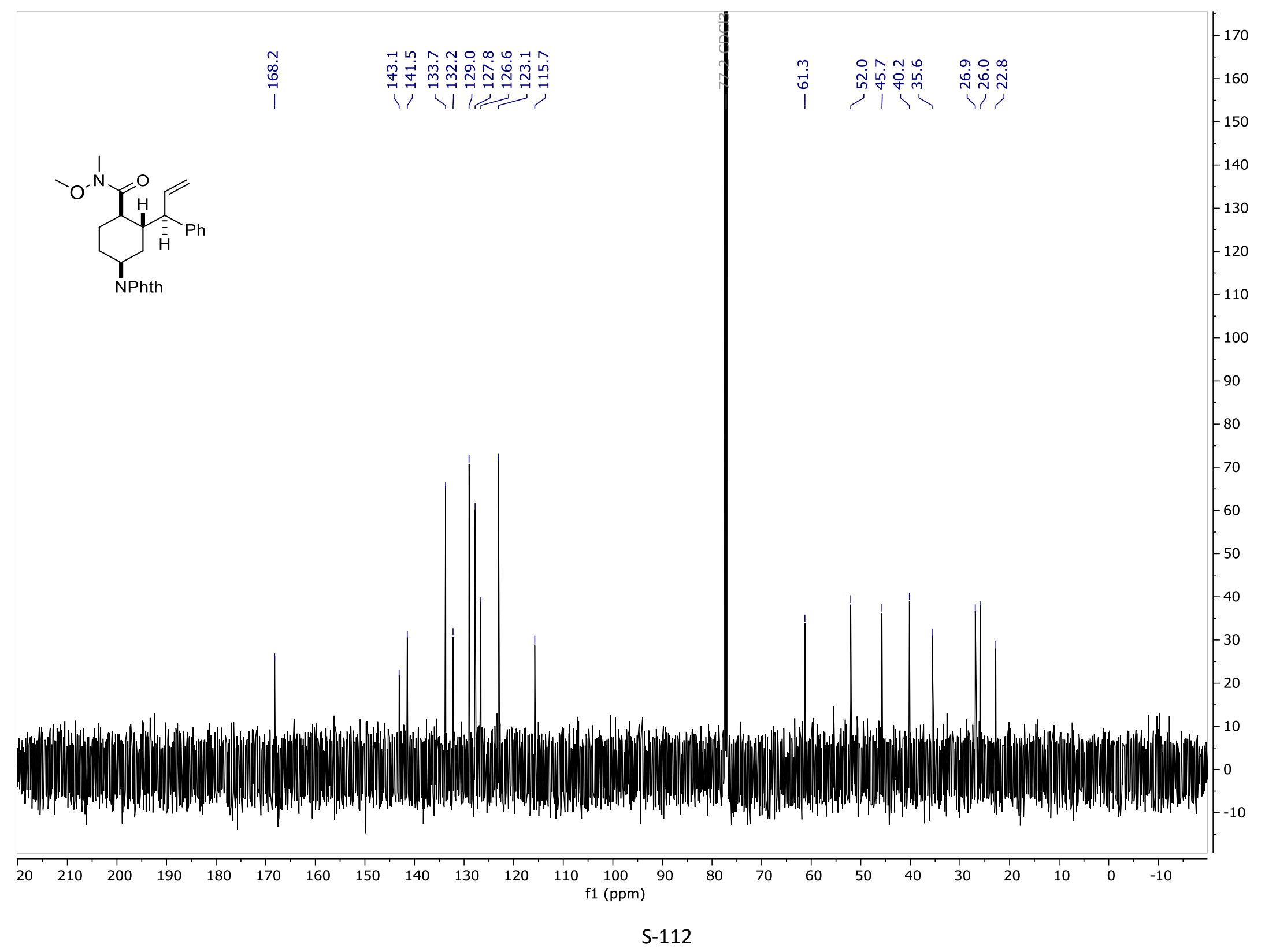


2-((1S,3S,4R)-3-((R)-1-(4-fluorophenyl)allyl)-4-(morpholine-4-carbonyl)cyclohexyl)isoindoline-1,3-dione (4l) 8:1 d.r. (600 MHz, CDCl $)$

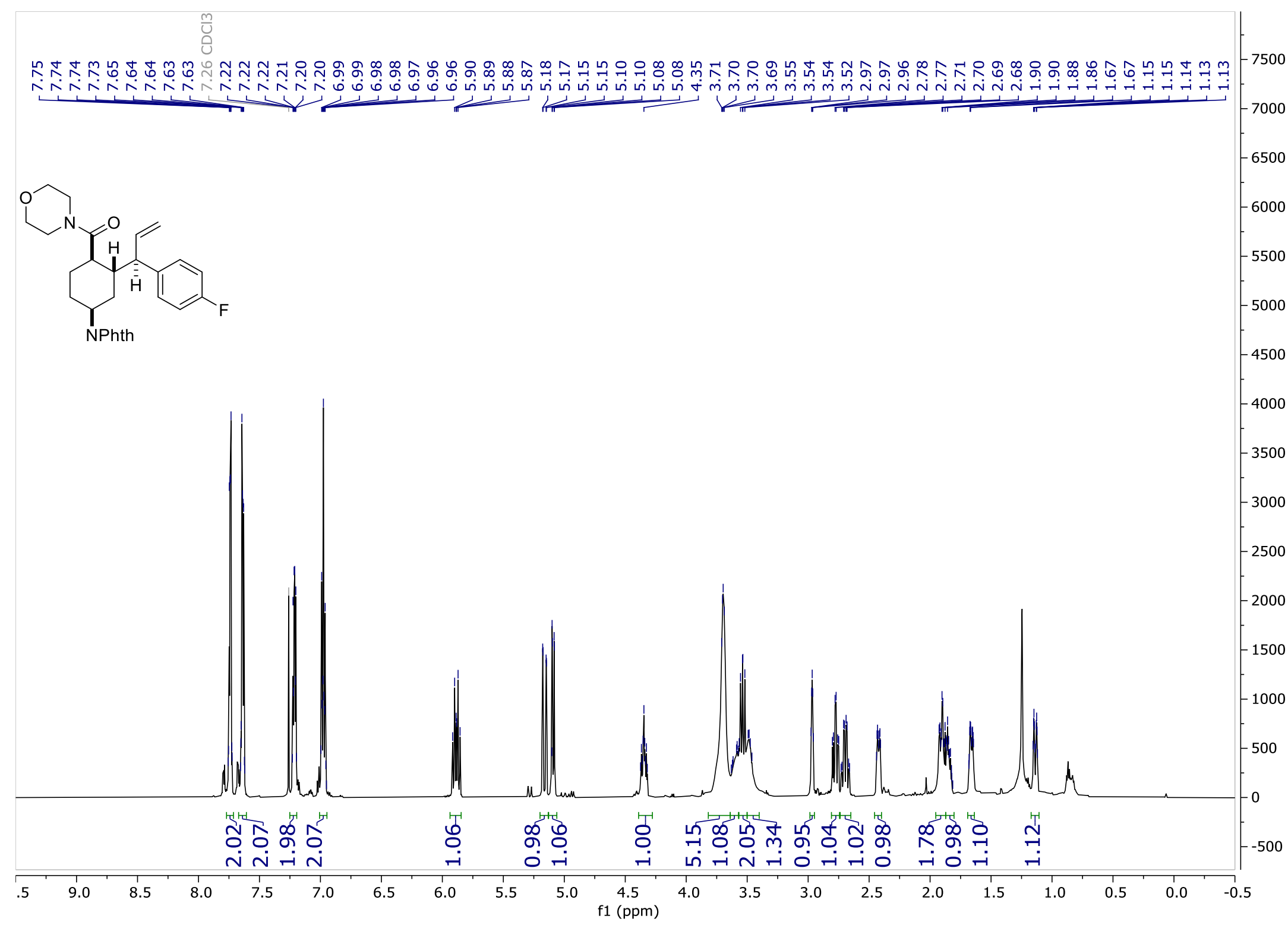




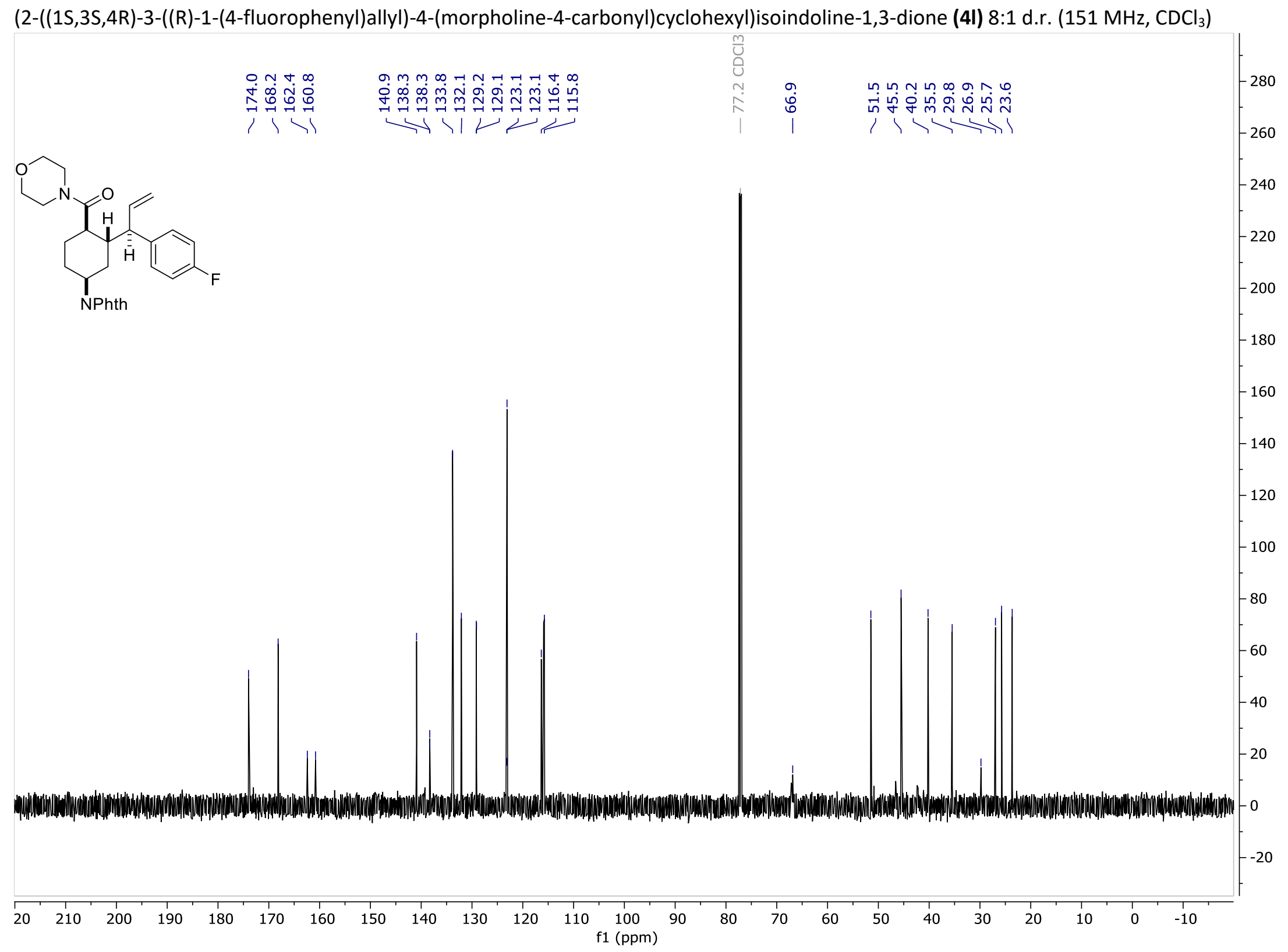

S-114 
2-((1S,3S,4R)-3-((R)-1-(4-fluorophenyl)allyl)-4-(morpholine-4-carbonyl)cyclohexyl)isoindoline-1,3-dione (4I) ${ }^{19} \mathrm{~F}$ Spectrum (565 MHz, CDCl 3$)$

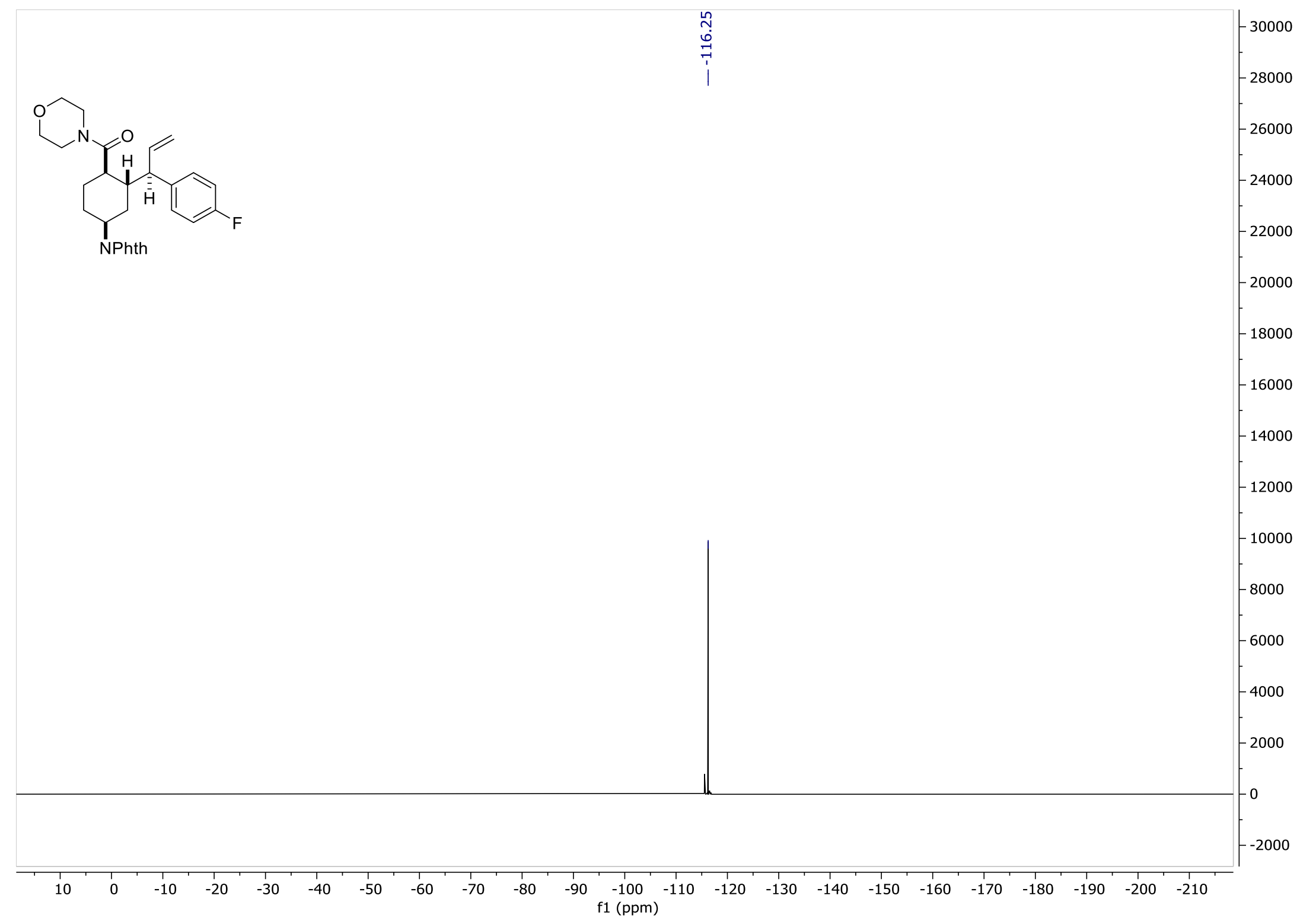


2-((1S,3S,4R)-3-((R)-1-(4-fluorophenyl)allyl)-4-(morpholine-4-carbonyl)cyclohexyl)isoindoline-1,3-dione (4I) HMBC

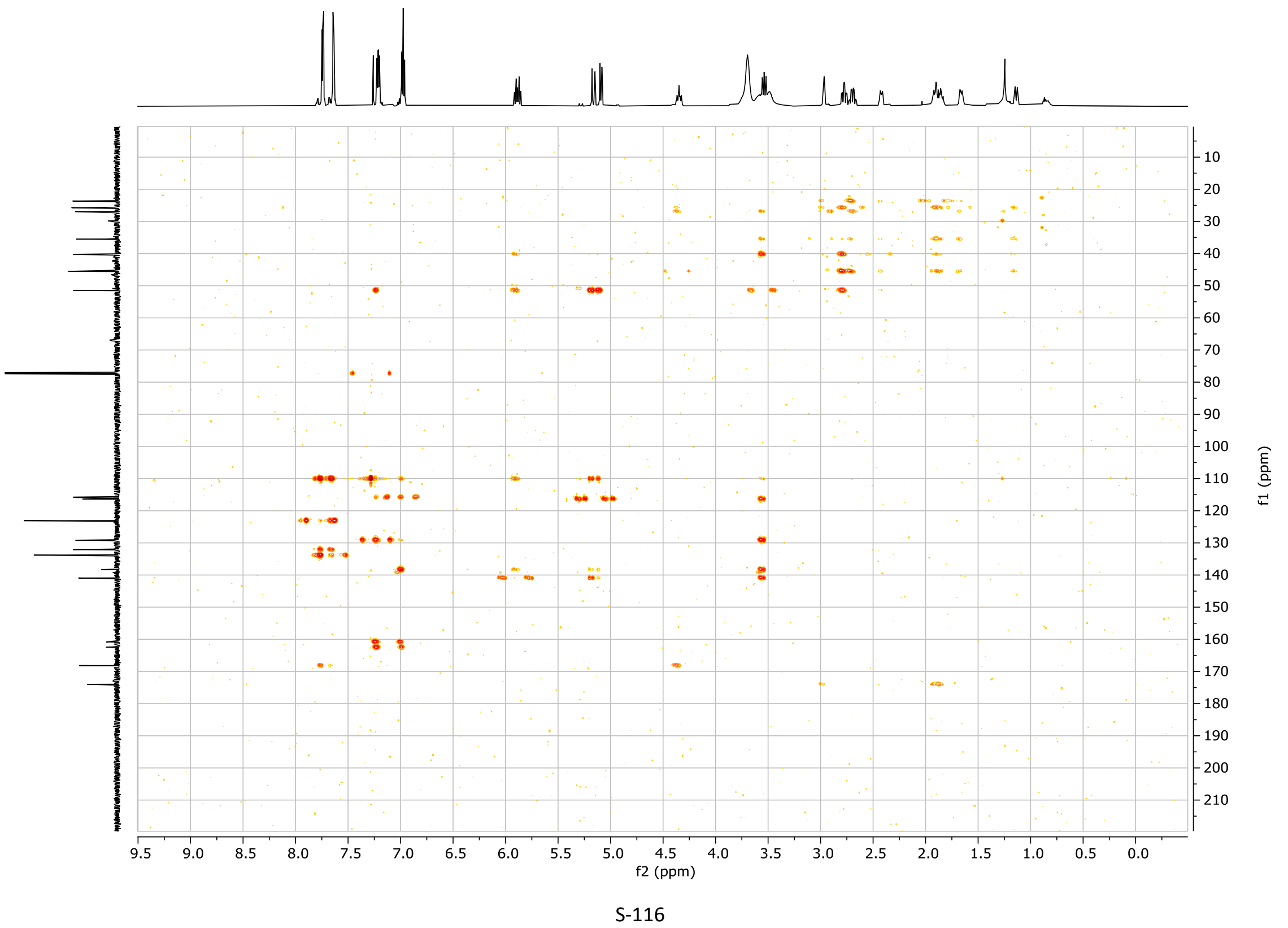


$(1 \mathrm{~S}, 2 \mathrm{~S}, 4 \mathrm{~S})-4-(1,3-$ dioxoisoindolin-2-yl)-2-((R)-1-(2-fluorophenyl)allyl)-N-methoxy-N-methylcyclohexane-1-carboxamide (4m) 5:1 d.r. (600 MHz, $\mathrm{CDCl}_{3}$ )

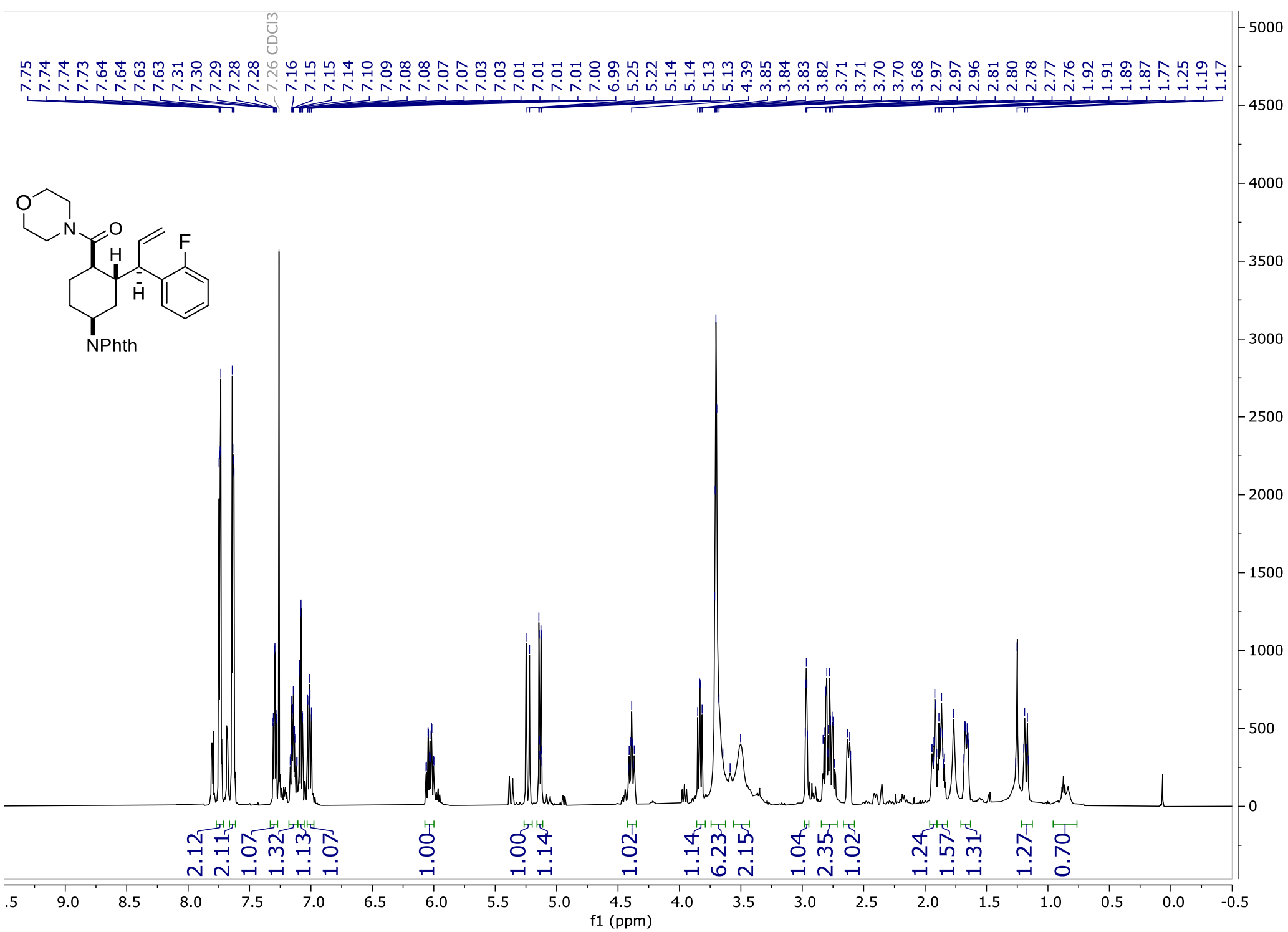


$(1 \mathrm{~S}, 2 \mathrm{~S}, 4 \mathrm{~S})-4-(1,3-$ dioxoisoindolin-2-yl)-2-((R)-1-(2-fluorophenyl)allyl)-N-methoxy-N-methylcyclohexane-1-carboxamide (4m) (151 MHz, CDCl 3 )

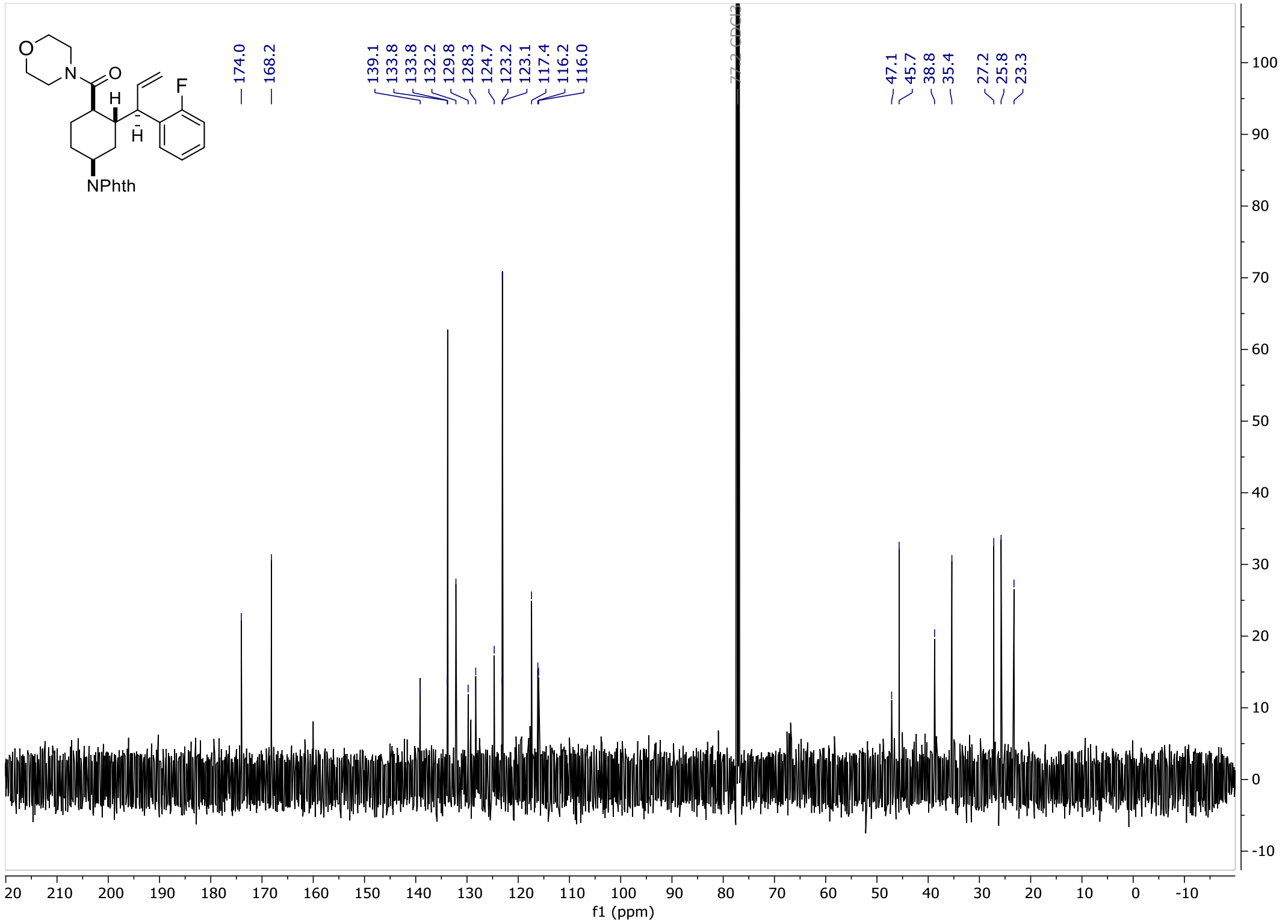


(1S,2S,4S)-4-(1,3-dioxoisoindolin-2-yl)-2-((R)-1-(2-fluorophenyl)allyl)-N-methoxy- $\mathrm{N}$-methylcyclohexane-1-carboxamide (4m) ${ }^{19} \mathrm{~F} \mathrm{NMR}$ ( 565MHz, $\mathrm{CDCl}_{3}$ )

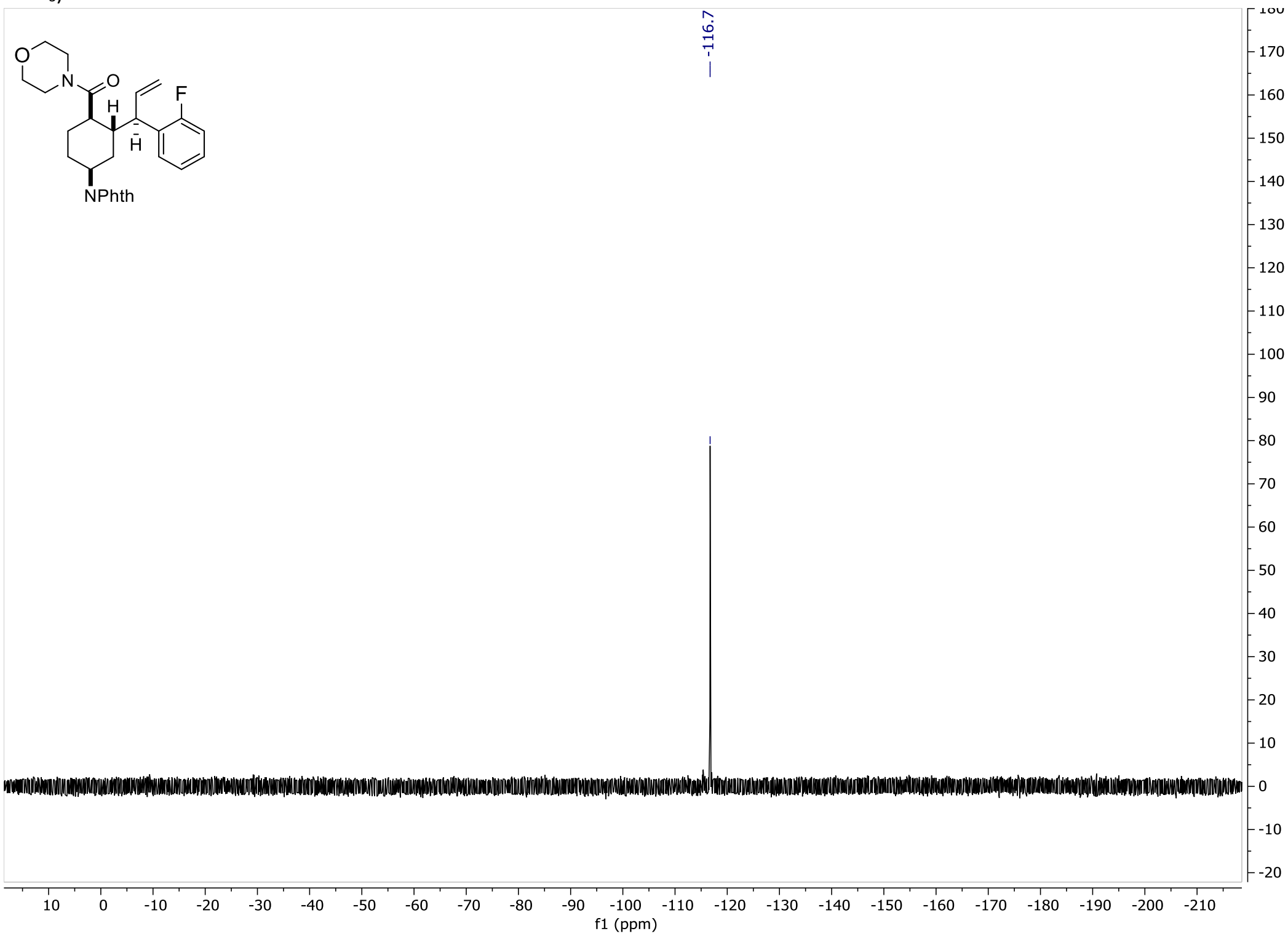


(1S,2S,4S)-4-(1,3-dioxoisoindolin-2-yl)-2-((R)-1-(2-fluorophenyl)allyl)-N-methoxy-N-methylcyclohexane-1-carboxamide (4m) HMBC

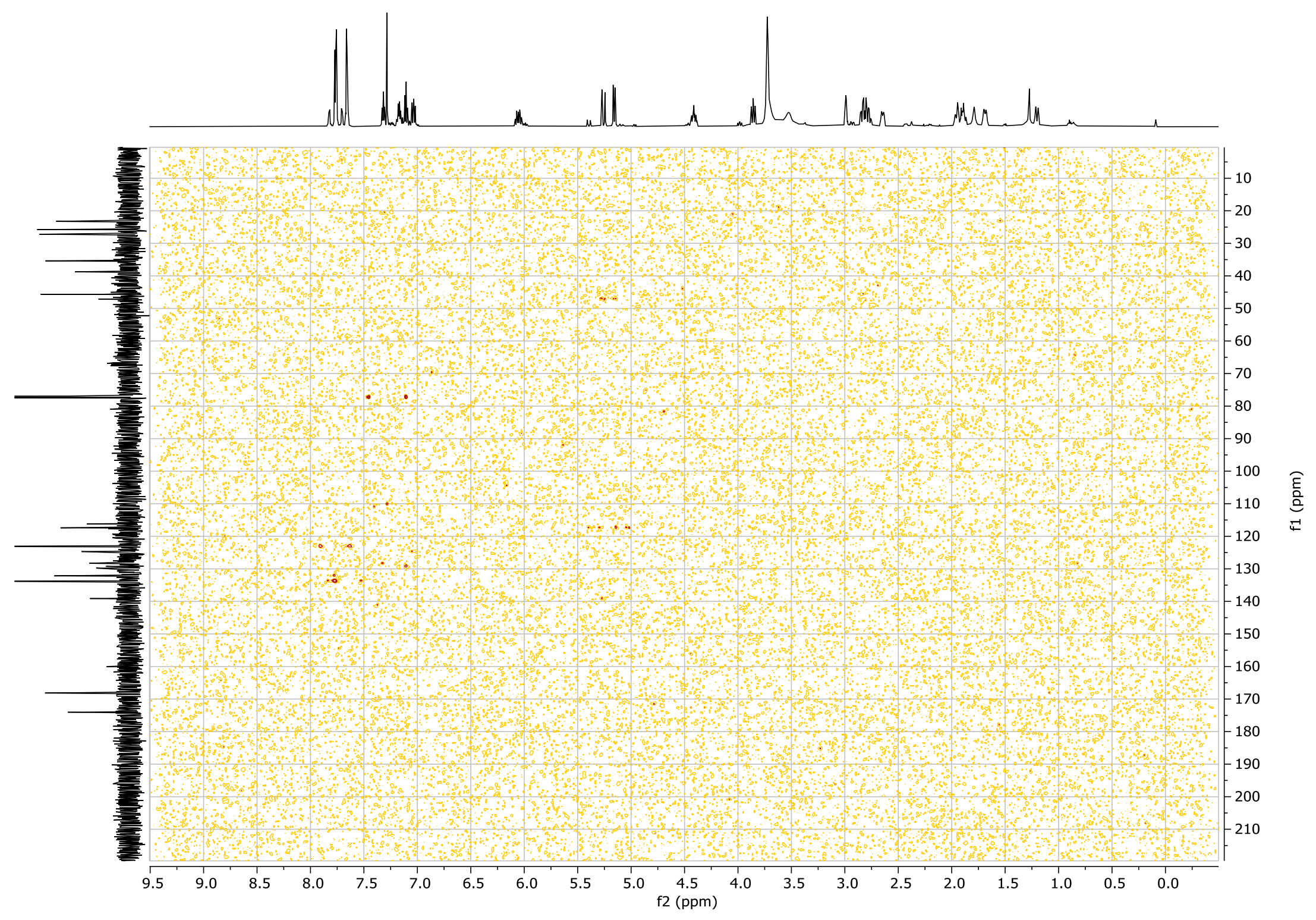


(1S,2S,4S)-4-(1,3-dioxoisoindolin-2-yl)-N-methoxy-2-((R)-1-(4-methoxyphenyl)allyl)-N-methylcyclohexane-1-carboxamide (4n) 8:1 d.r. (600 MHz, $\mathrm{CDCl}_{3}$ )

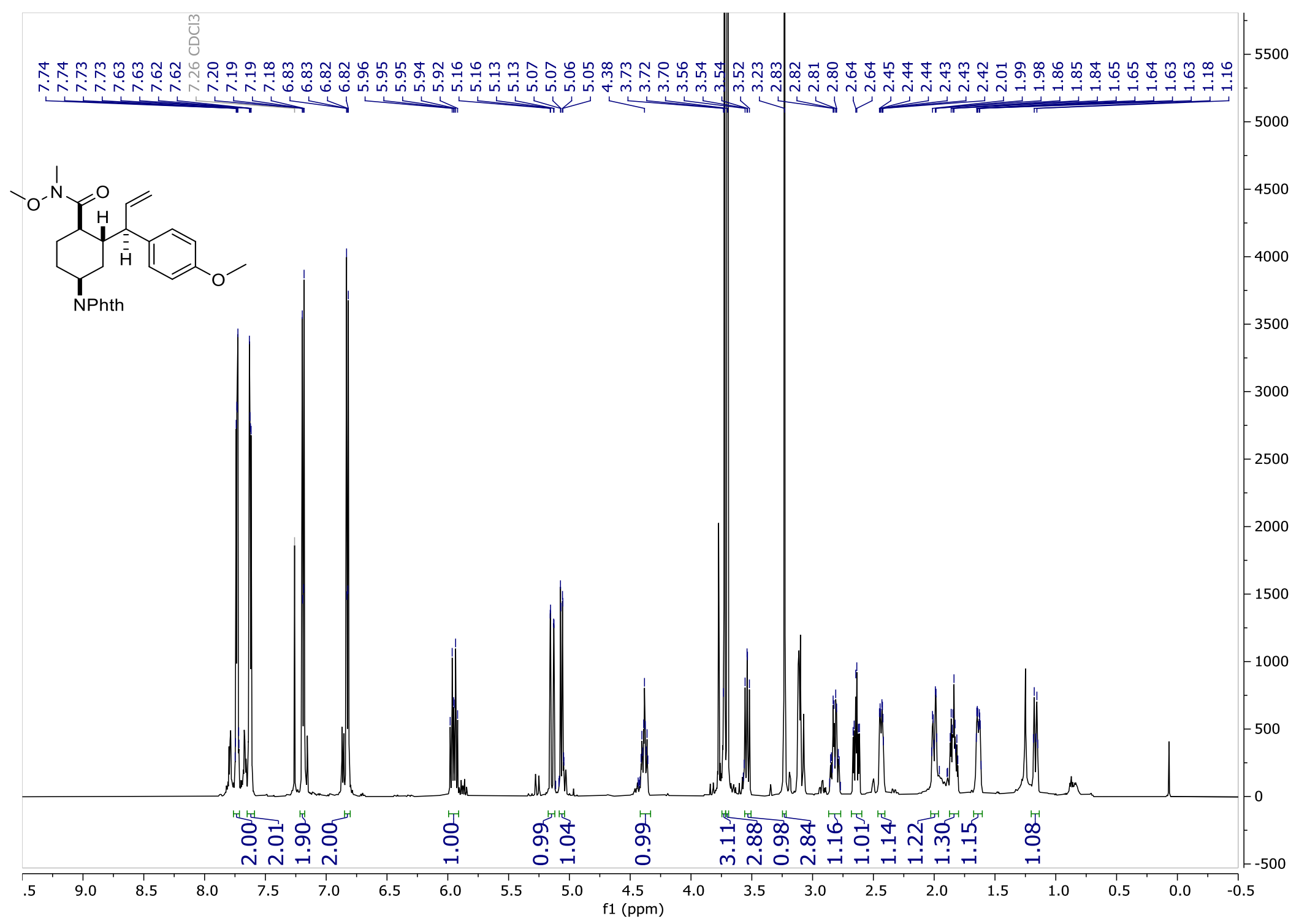


(1S,2S,4S)-4-(1,3-dioxoisoindolin-2-yl)-N-methoxy-2-((R)-1-(4-methoxyphenyl)allyl)-N-methylcyclohexane-1-carboxamide (4n) (151 MHz, CDCl 3 )

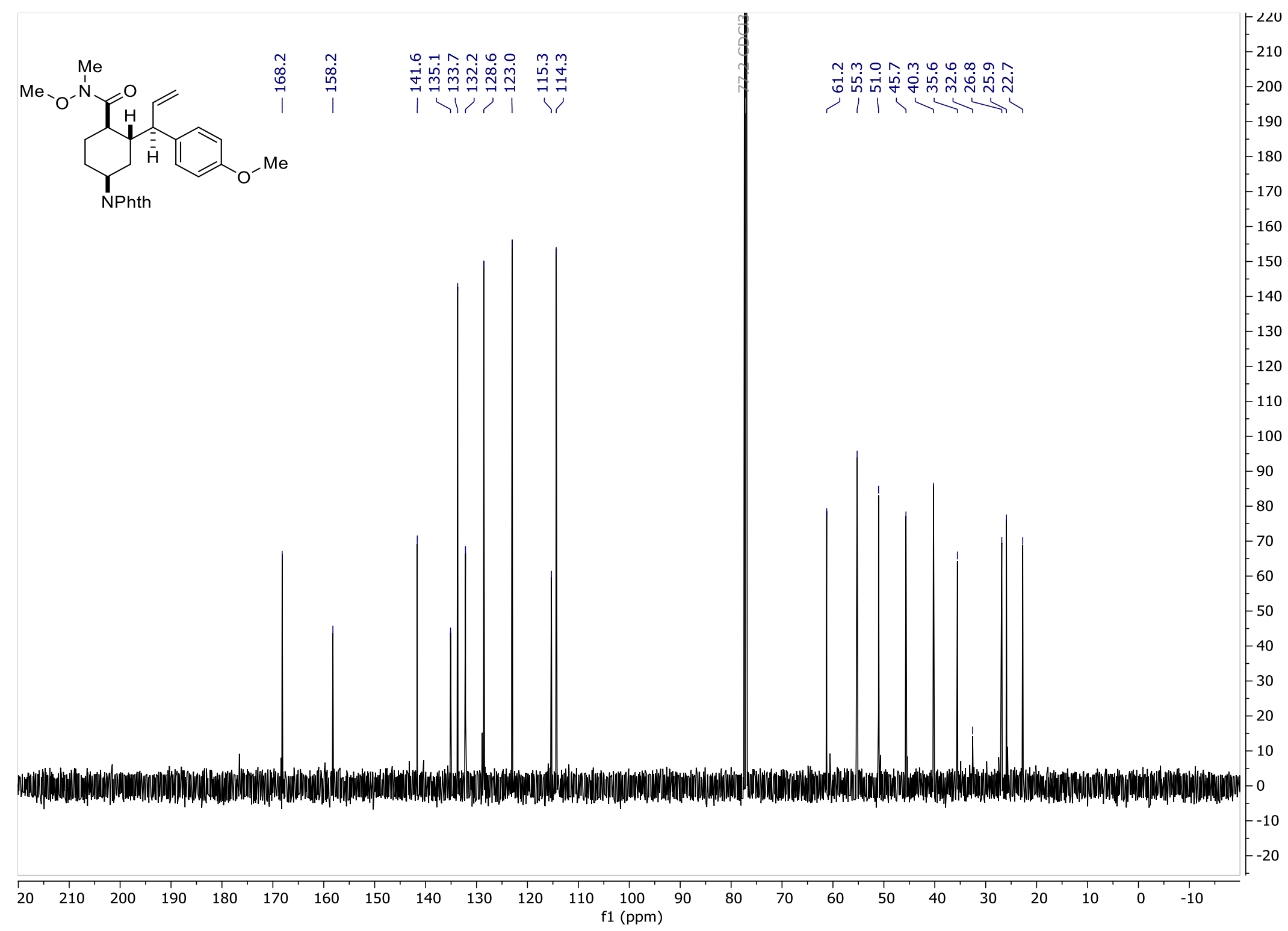


2-((1S,3S,4S)-3-((R)-1-(4-(1,3-dioxolan-2-yl)phenyl)allyl)-4-(morpholine-4-carbonyl)cyclohexyl)isoindoline-1,3-dione (4o) 8:1 d.r. (600 MHz, CDCl $)$

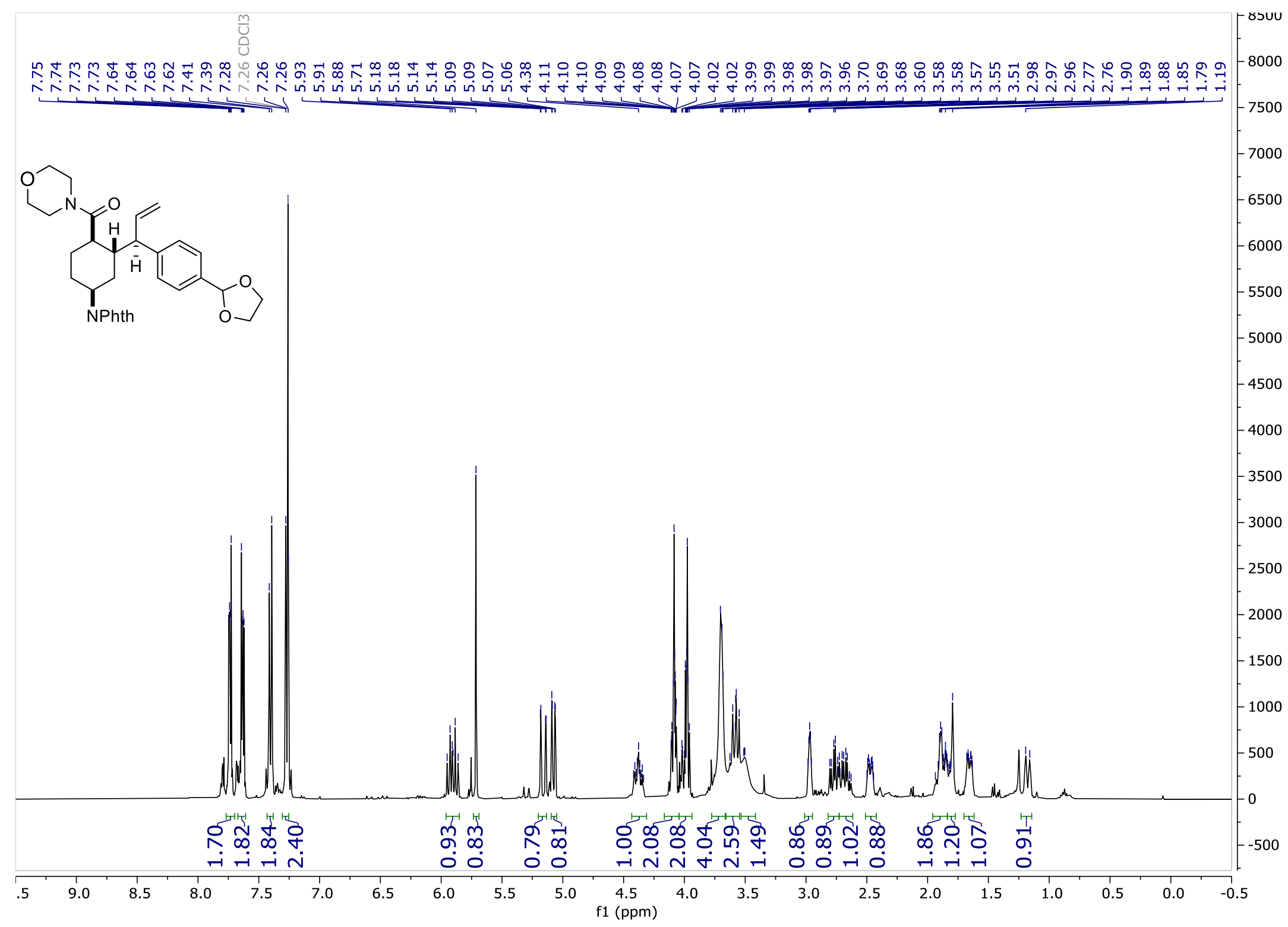


2-((1S,3S,4S)-3-((R)-1-(4-(1,3-dioxolan-2-yl)phenyl)allyl)-4-(morpholine-4-carbonyl)cyclohexyl)isoindoline-1,3-dione (40) 8:1 d.r. (151 MHz, CDCl 3 )
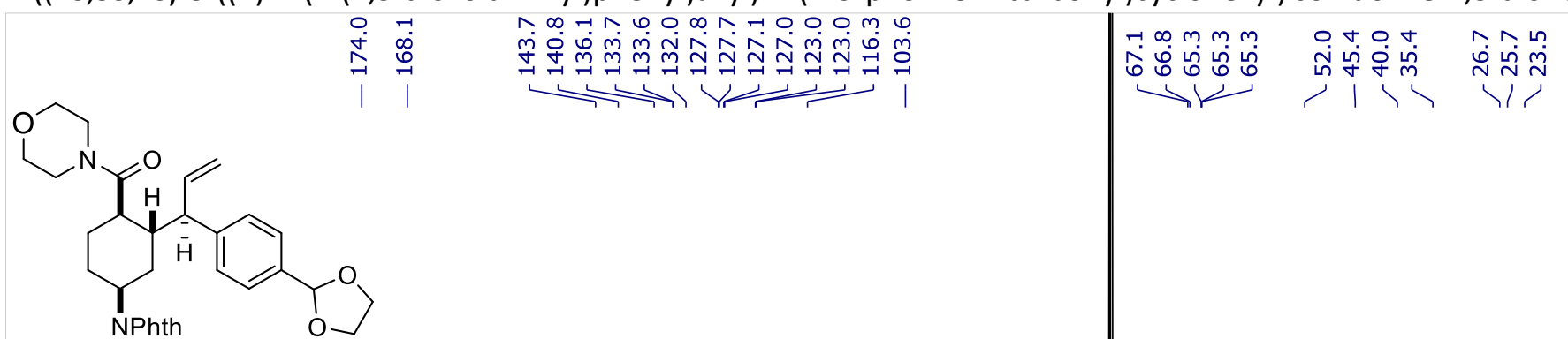

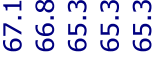

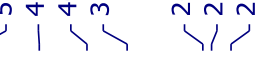

1500

1400

$-1300$

1200

1100

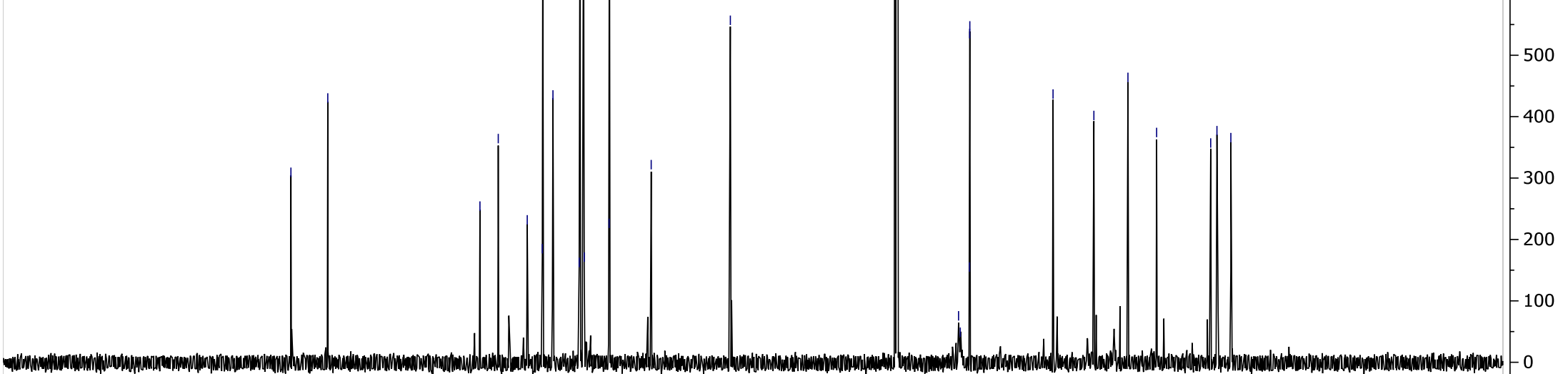

WWNW

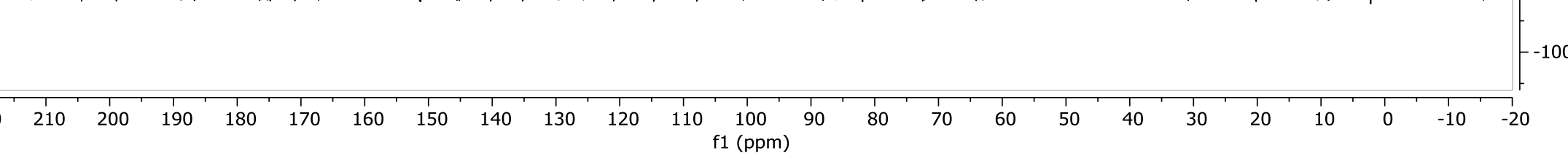


(1S,2S,4S)-N-benzyl-4-(1,3-dioxoisoindolin-2-yl)-2-((R)-1-phenylallyl)cyclohexane-1-carboxamide (5) 8.2:1 d.r. (600 MHz, CDCl $)$

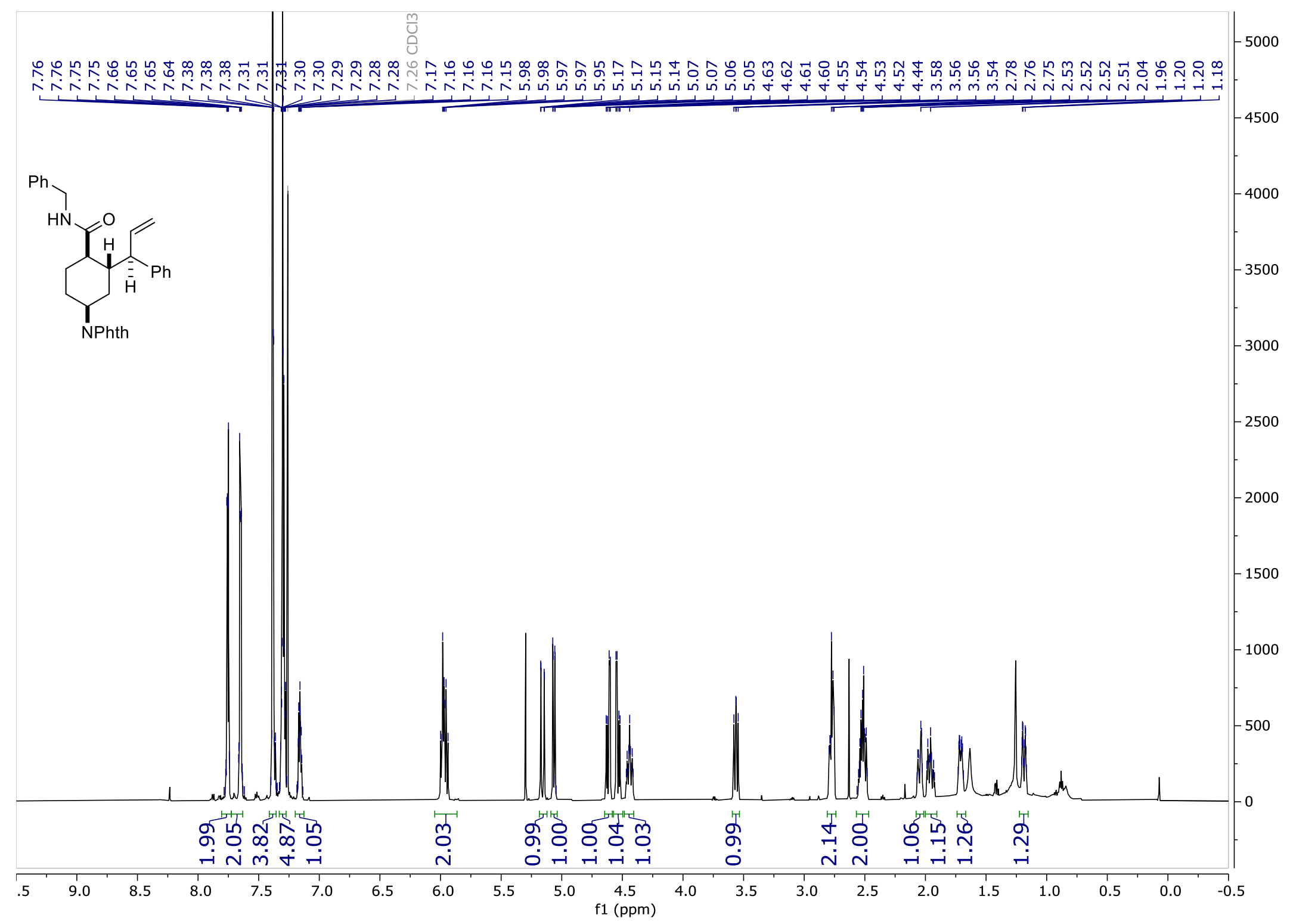


(1S,2S,4S)-N-benzyl-4-(1,3-dioxoisoindolin-2-yl)-2-((R)-1-phenylallyl)cyclohexane-1-carboxamide (5) (151 Mhz, $\left.\mathrm{CDCl}_{3}\right)$

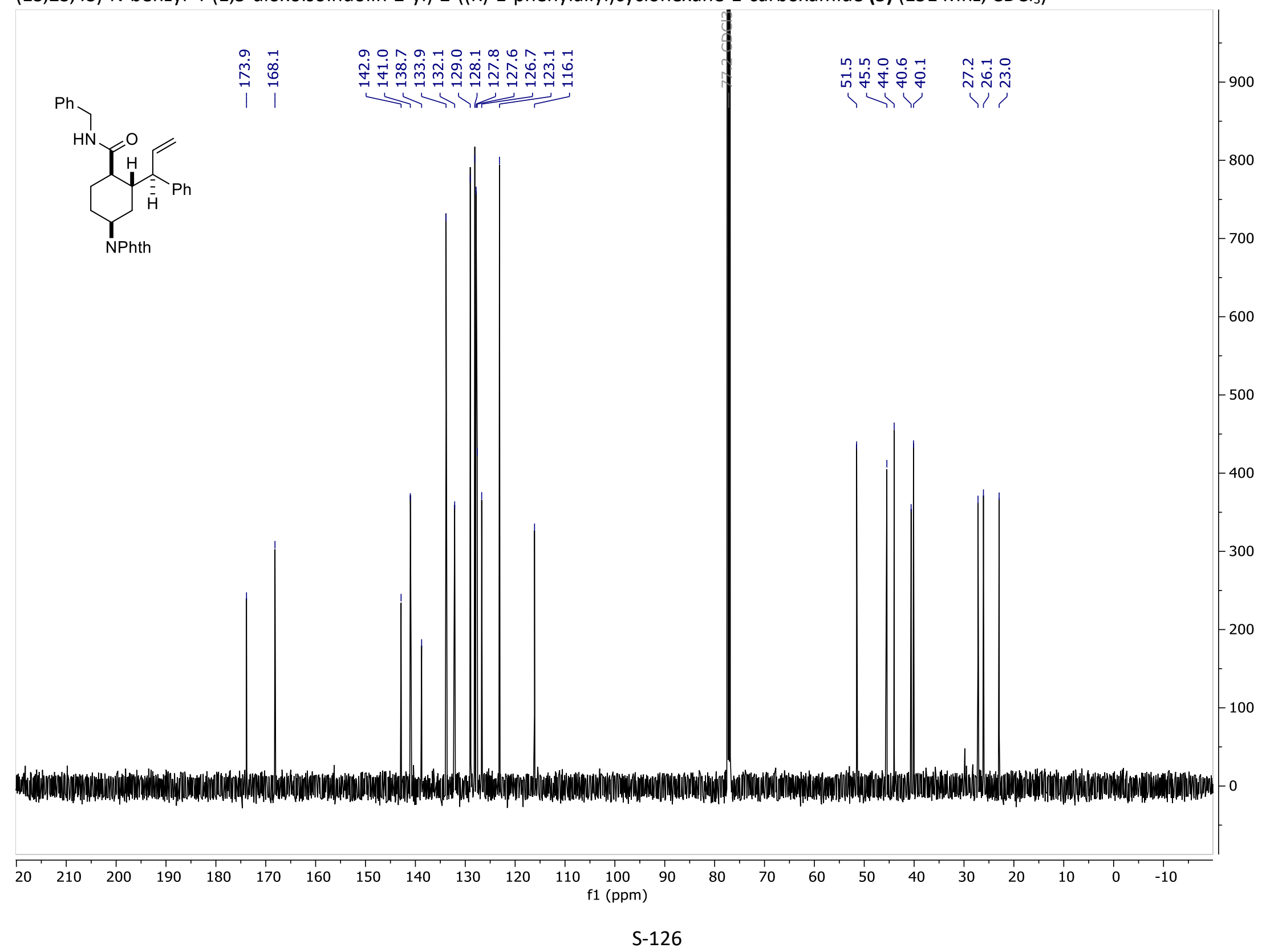


methyl (1S,2S,4S)-4-(1,3-dioxoisoindolin-2-yl)-2-((R)-1-phenylallyl)cyclohexane-1-carboxylate (6) 3.2:1 d.r. $\left(600 \mathrm{MHz} \mathrm{CDCl}_{3}\right)$

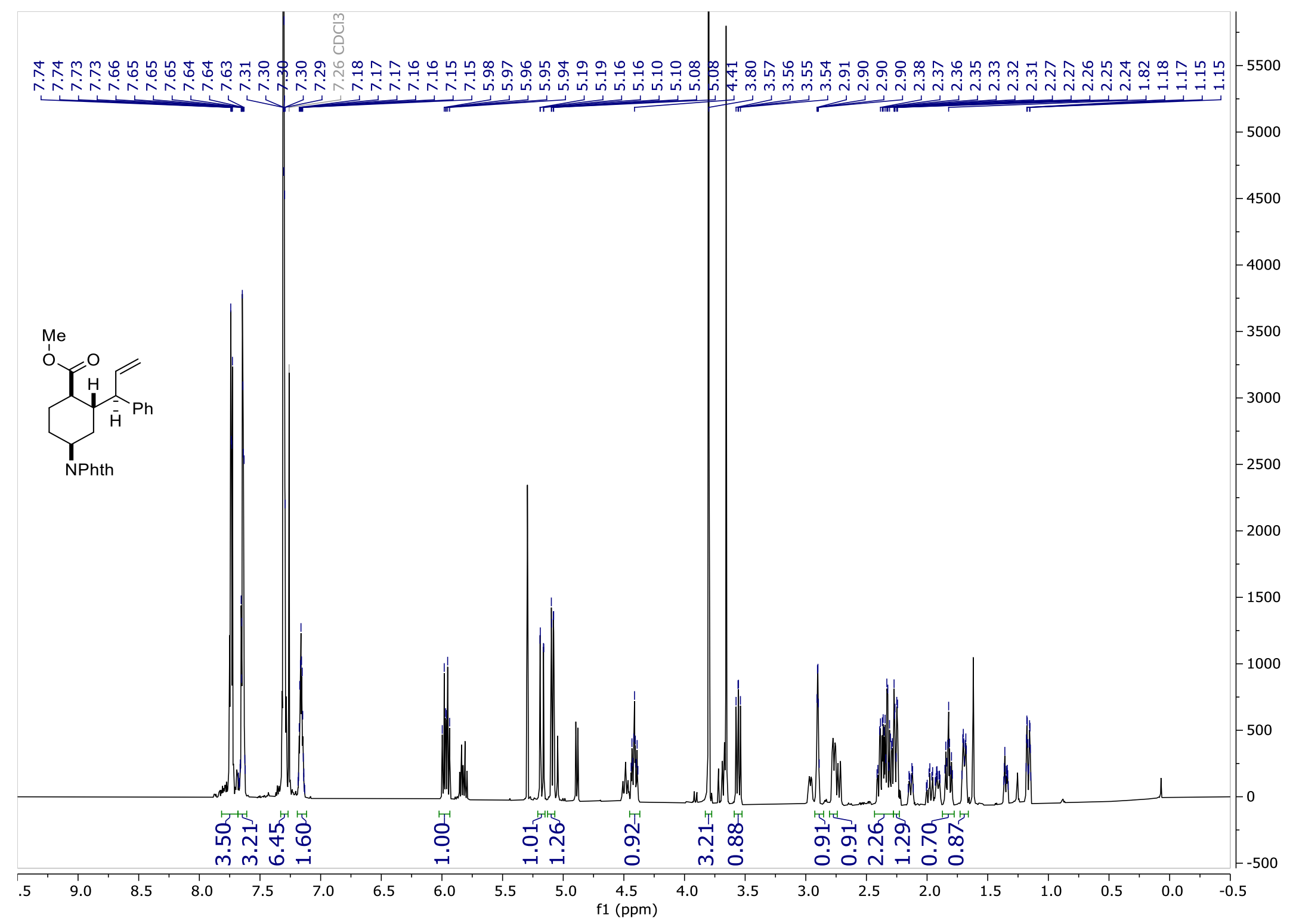


methyl (1S,2S,4S)-4-(1,3-dioxoisoindolin-2-yl)-2-((R)-1-phenylallyl)cyclohexane-1-carboxylate (6) (151 MHz, $\left.\mathrm{CDCl}_{3}\right)$

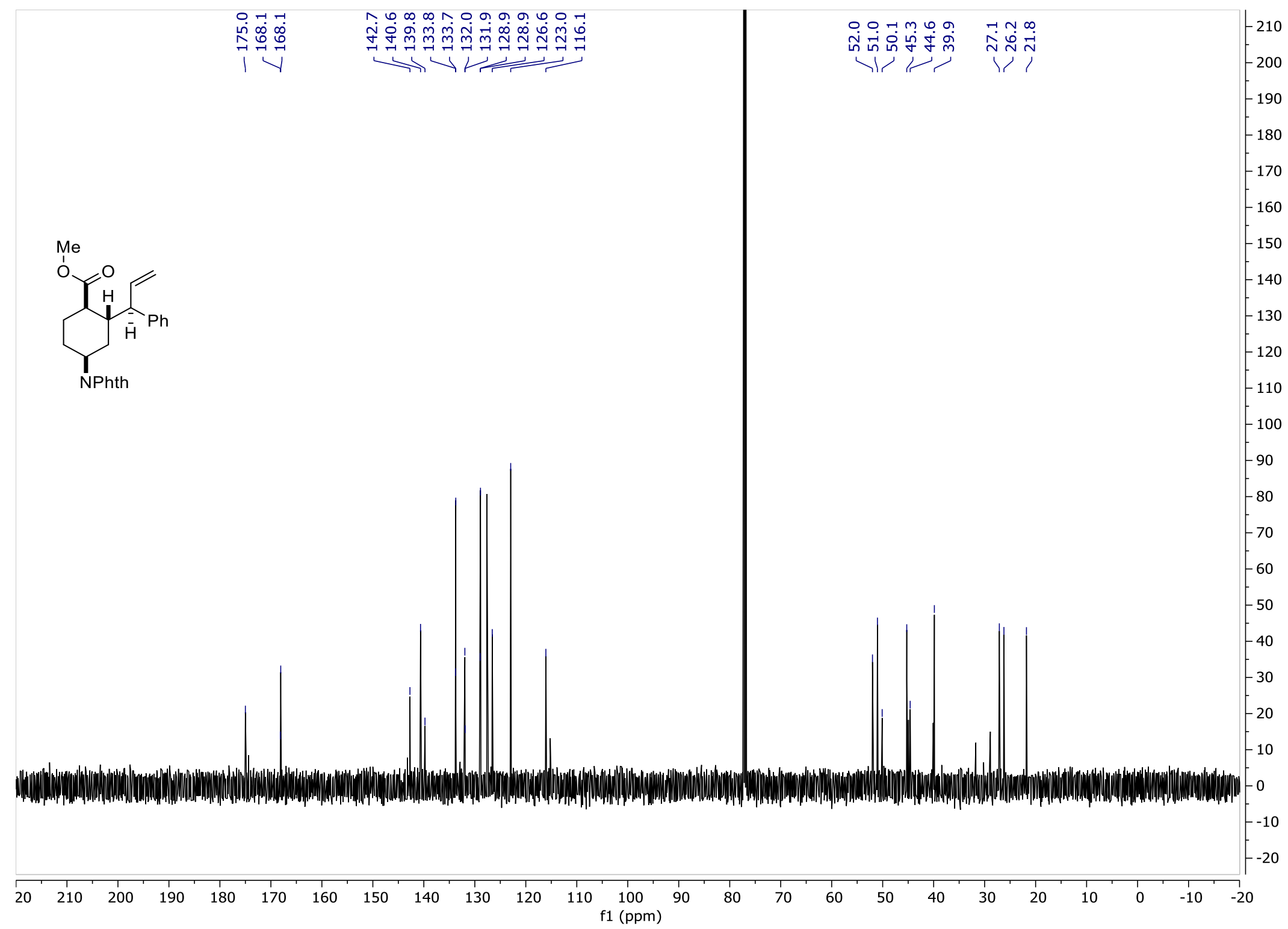


2-(hydroxymethyl)-N-((1S,3S,4R)-4-(hydroxymethyl)-3-((R)-1-phenylallyl)cyclohexyl)benzamide (7) 5.2:1 d.r. $\left(600 \mathrm{MHz}^{\mathrm{CDCl}} \mathrm{CD}_{3}\right.$

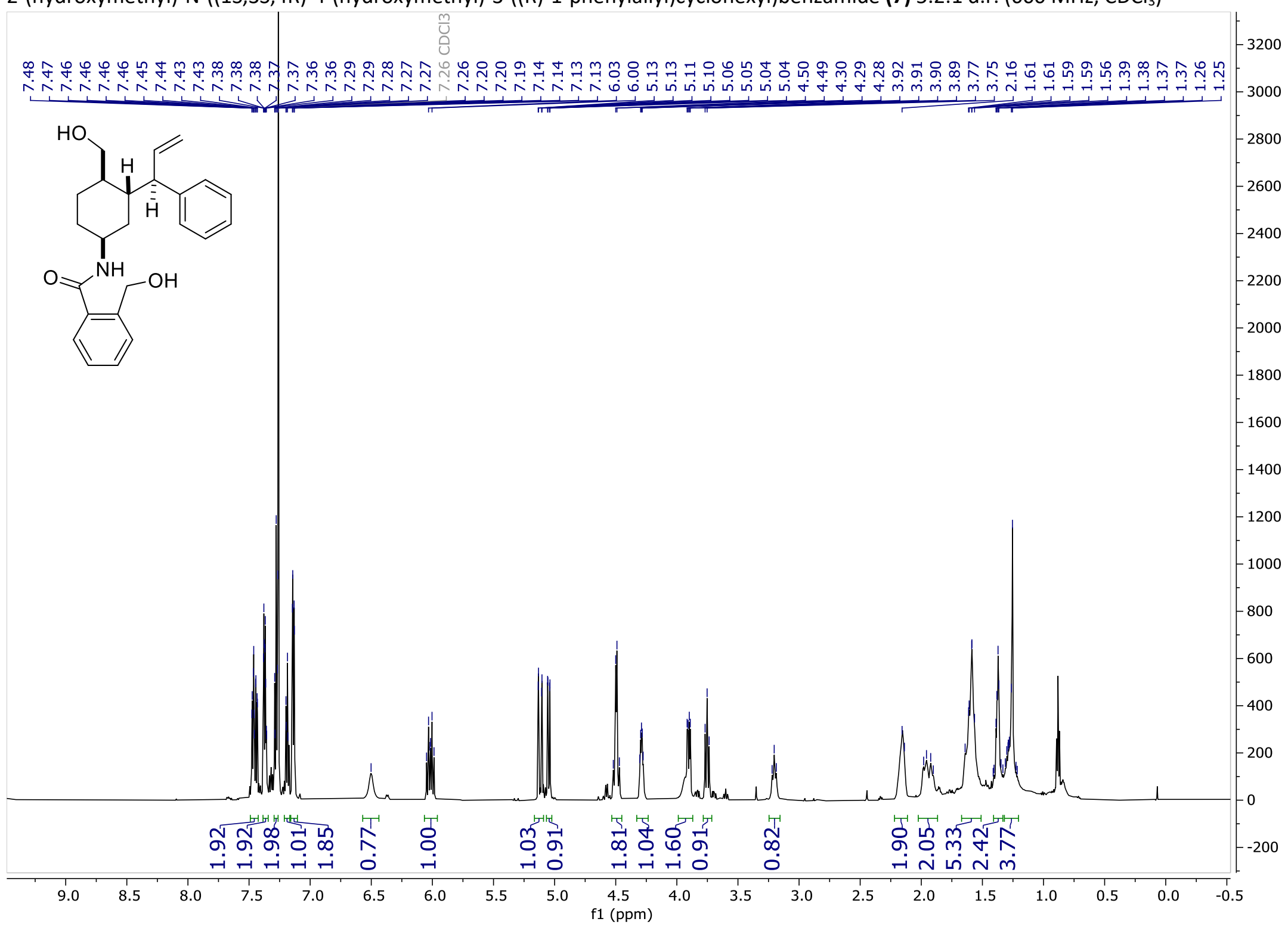


2-(hydroxymethyl)-N-((1S,3S,4R)-4-(hydroxymethyl)-3-((R)-1-phenylallyl)cyclohexyl)benzamide (7) (151 MHz, $\left.\mathrm{CDCl}_{3}\right)$

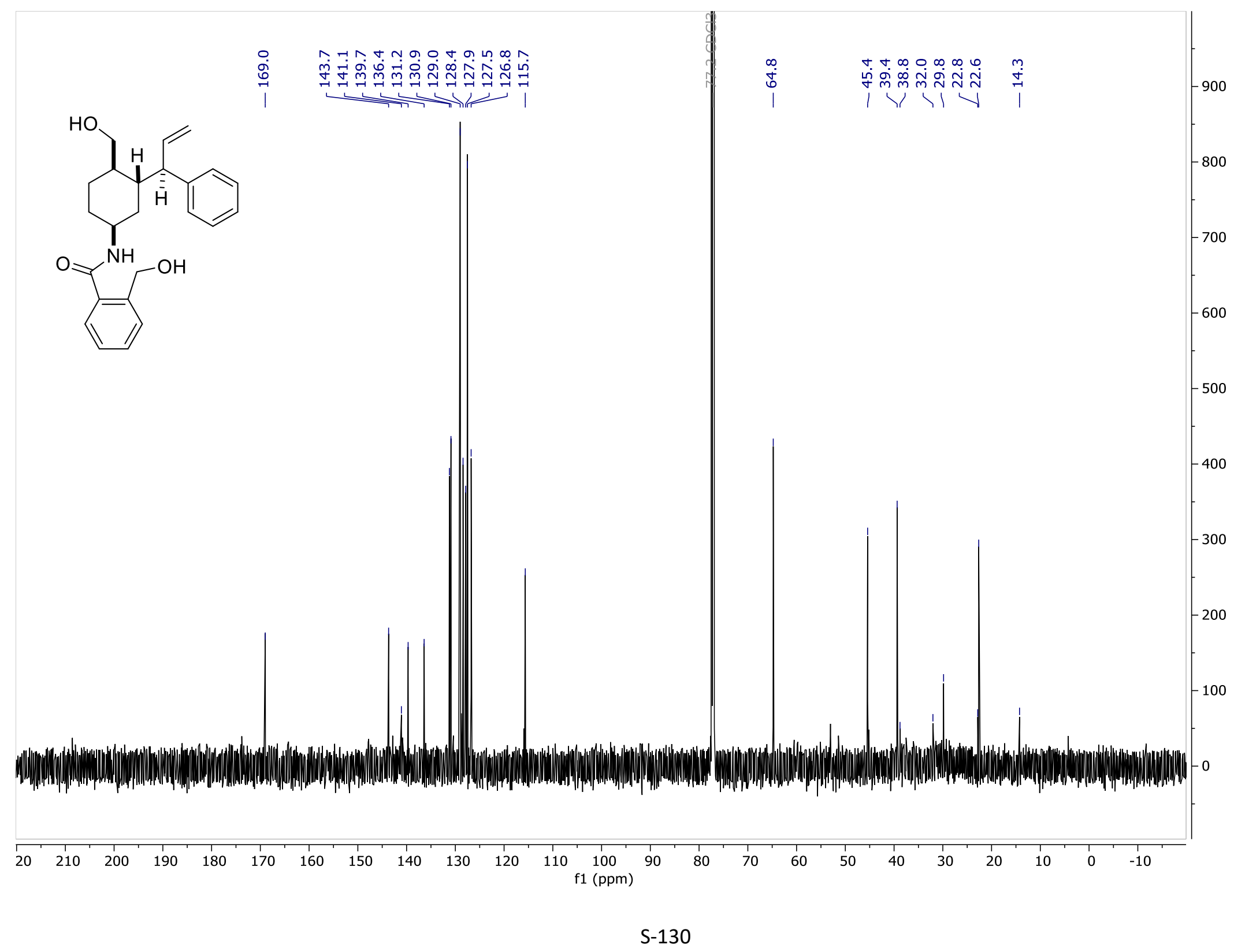


$\mathrm{N}-\left((1 \mathrm{~S}, 3 \mathrm{~S}, 4 \mathrm{~S})-4-\left(\right.\right.$ morpholine-4-carbonyl)-3-((R)-1-phenylallyl)cyclohexyl)methanesulfonamide $(8){ }^{1} \mathrm{H}$ Spectrum 7:1 conformer ratio (600 MHz, $\left.\mathrm{CDCl}_{3}\right)$

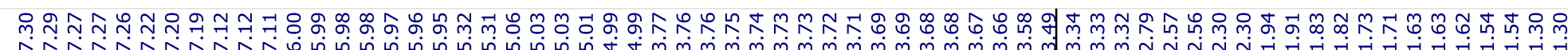

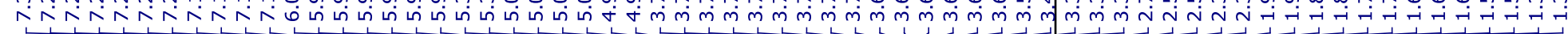

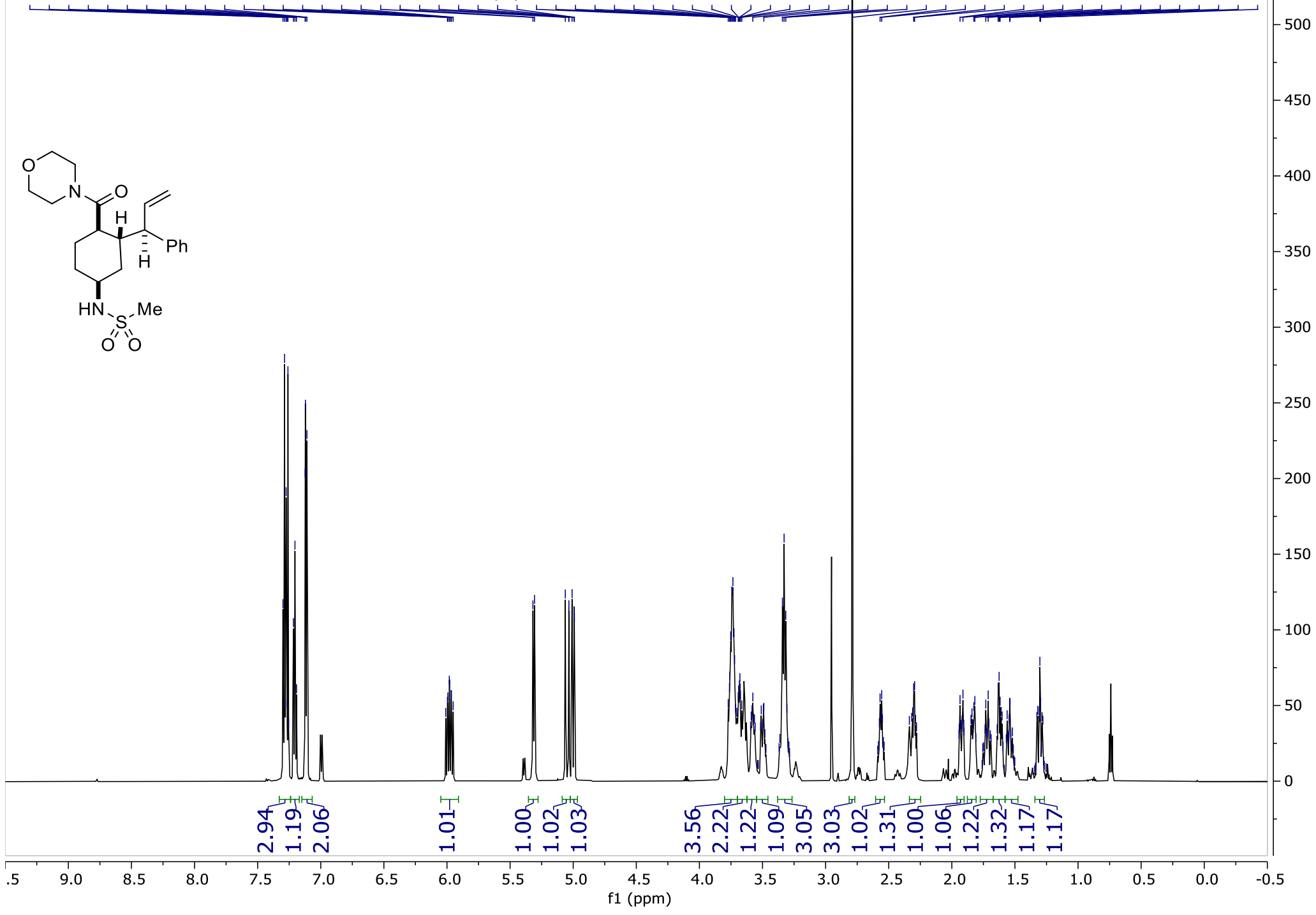


$\mathrm{N}-\left((1 \mathrm{~S}, 3 \mathrm{~S}, 4 \mathrm{~S})-4-\left(\right.\right.$ morpholine-4-carbonyl)-3-((R)-1-phenylallyl)cyclohexyl)methanesulfonamide (8) (151 MHz, $\left.\mathrm{CDCl}_{3}\right)$

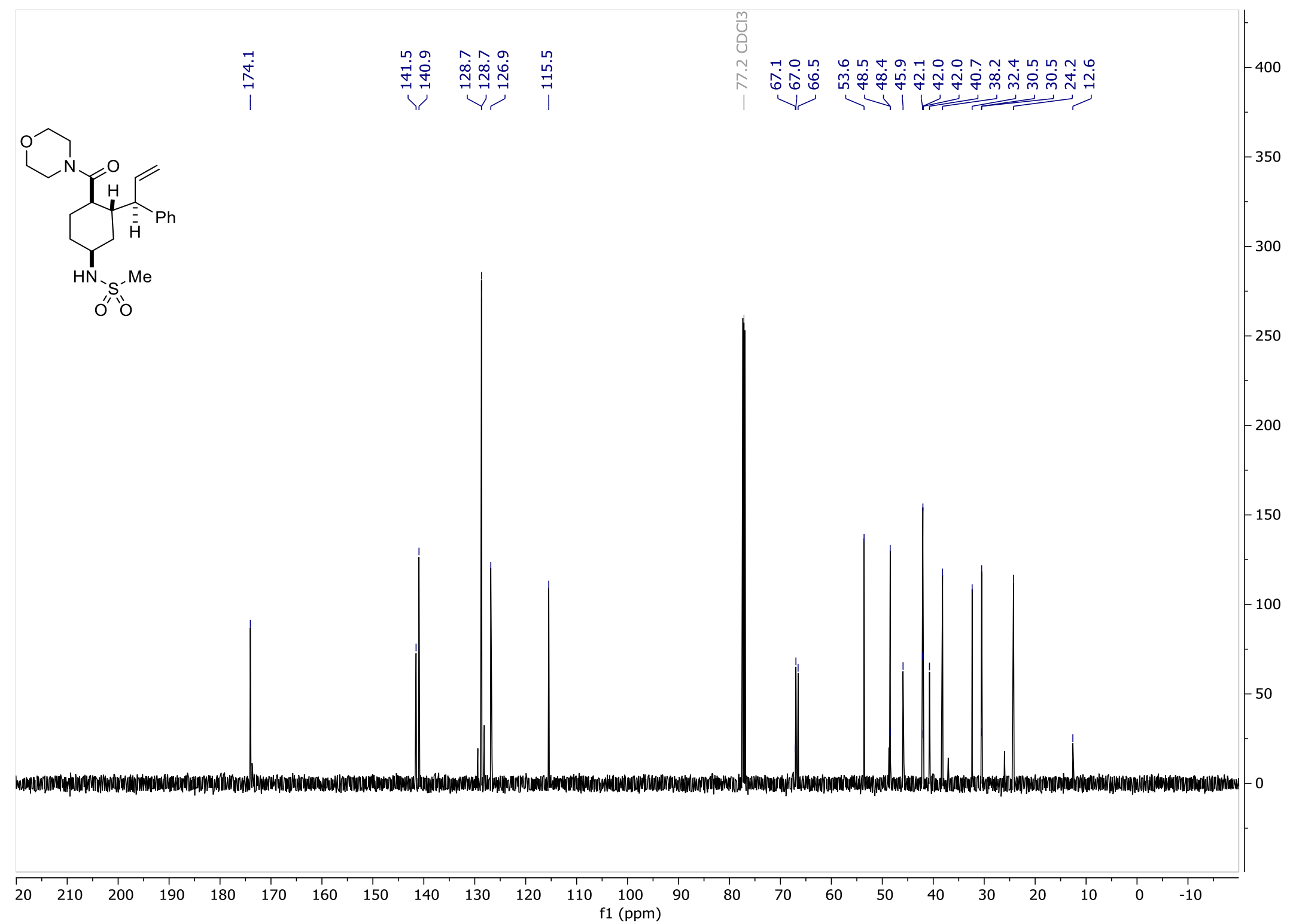


$\mathrm{N}-\left((1 \mathrm{~S}, 3 \mathrm{~S}, 4 \mathrm{~S})-4-\left(\right.\right.$ morpholine-4-carbonyl)-3-((R)-1-phenylallyl)cyclohexyl)methanesulfonamide $(8)^{1} \mathrm{H} \mathrm{NMR}$ at $-50{ }^{\circ} \mathrm{C},\left(500 \mathrm{MHz}, \mathrm{CDCl}_{3}\right)$

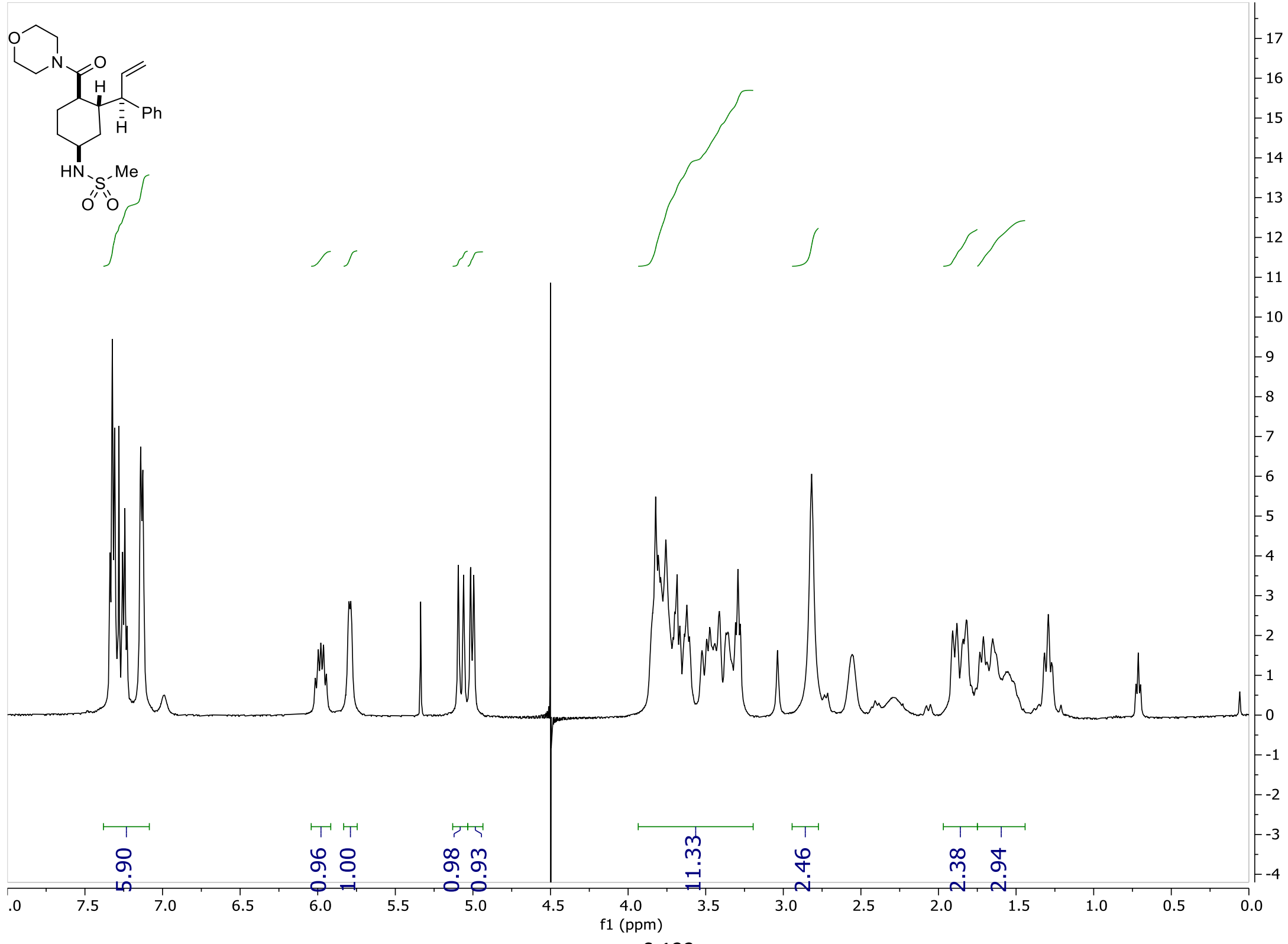

S-133 
$\mathrm{N}-\left((1 \mathrm{~S}, 3 \mathrm{~S}, 4 \mathrm{~S})-4-\left(\right.\right.$ morpholine-4-carbonyl)-3-((R)-1-phenylpropyl)cyclohexyl)methanesulfonamide (9) ${ }^{1} \mathrm{H}$ Spectrum $\left(400 \mathrm{MHz}, \mathrm{CDCl}_{3}\right)$

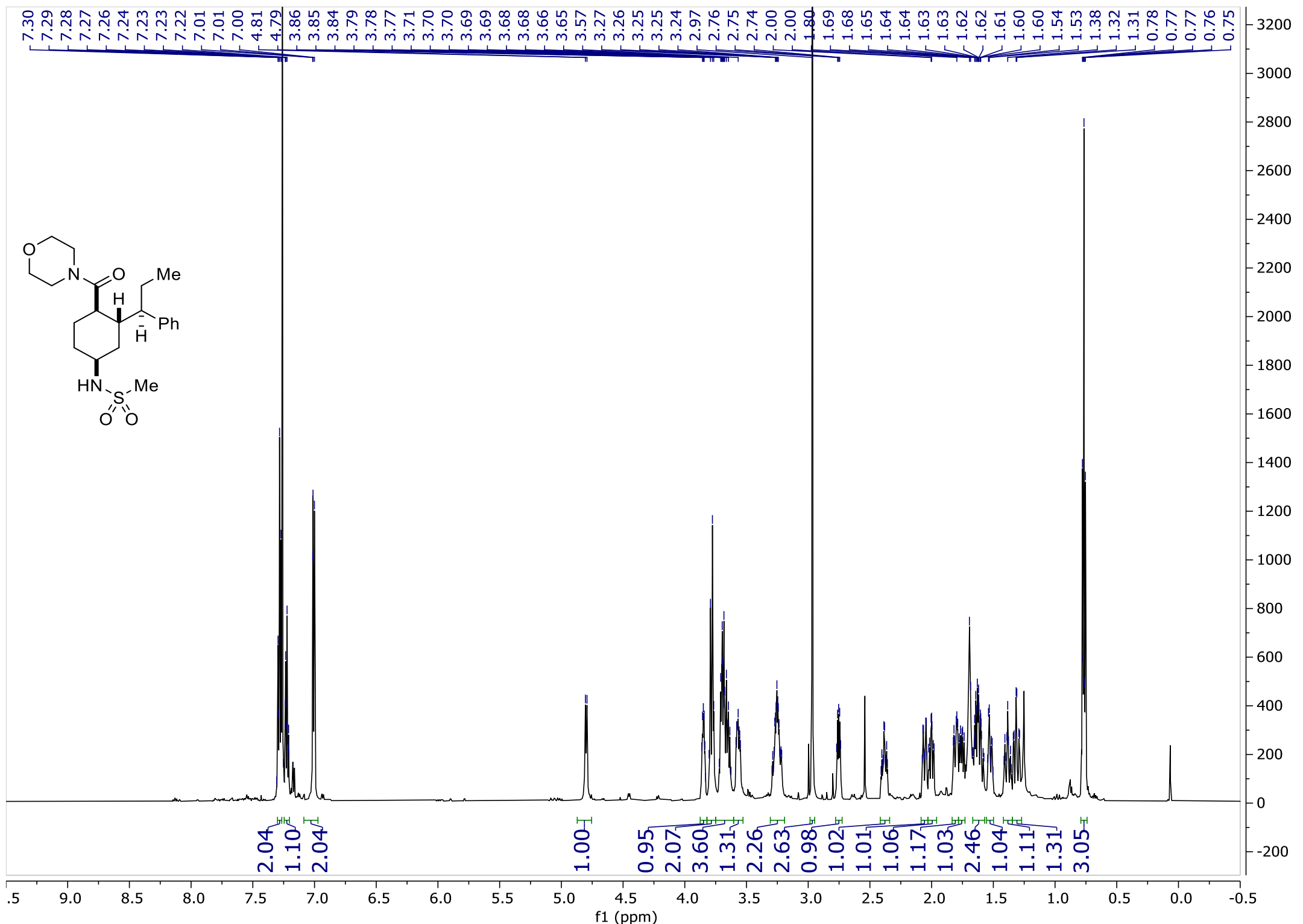


$\mathrm{N}-\left((1 \mathrm{~S}, 3 \mathrm{~S}, 4 \mathrm{~S})-4-\left(\right.\right.$ morpholine-4-carbonyl)-3-((R)-1-phenylpropyl)cyclohexyl)methanesulfonamide (9) ${ }^{13} \mathrm{C}$ Spectrum $(151 \mathrm{MHz}, \mathrm{CDCl} 3)$

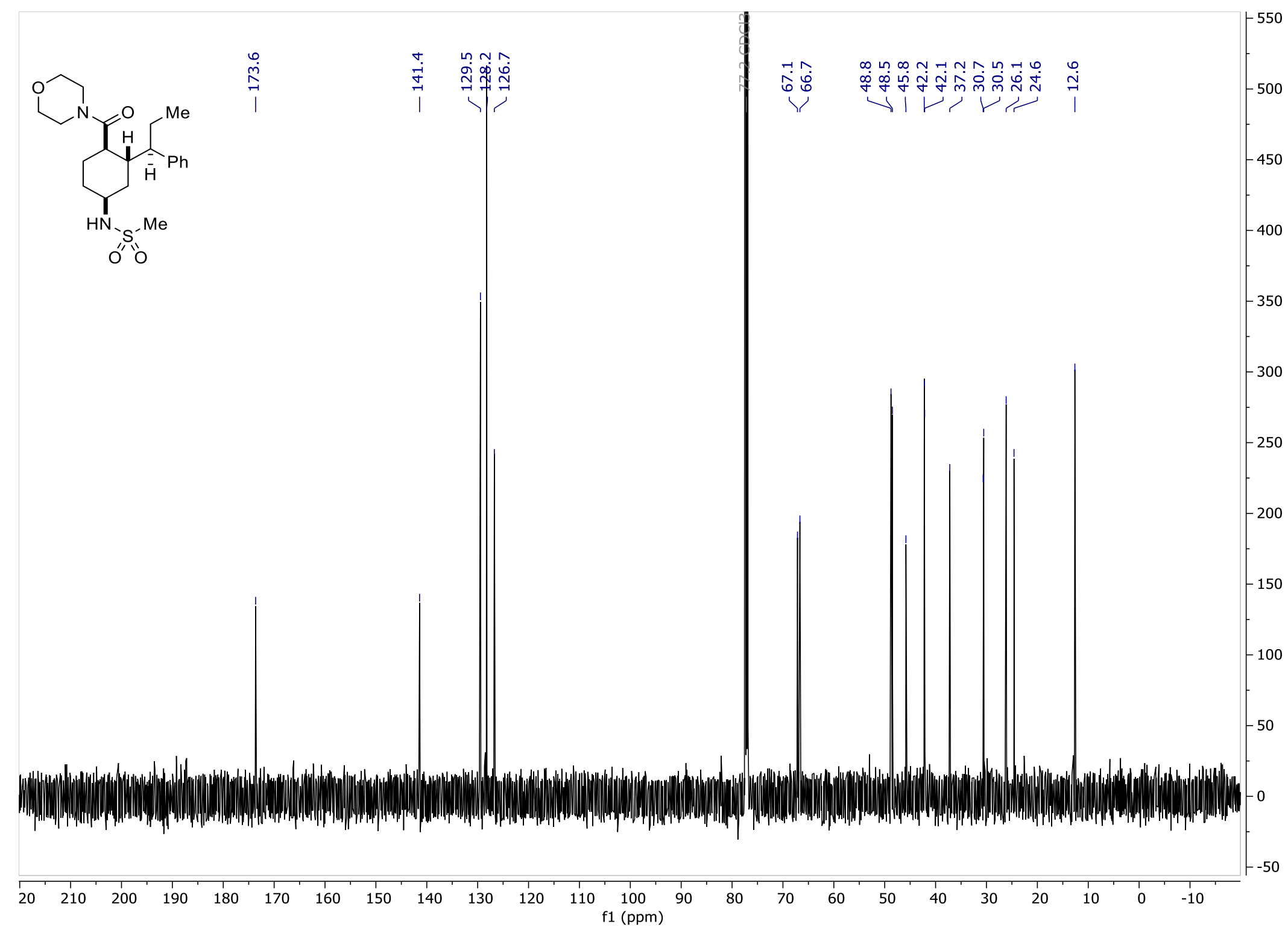


ethyl (R,E)-4-((1S,2S,5S)-5-(methylsulfonamido)-2-(morpholine-4-carbonyl)cyclohexyl)-4-phenylbut-2-enoate (10) ${ }^{1} \mathrm{H} \mathrm{Spectrum} \mathrm{(600} \mathrm{MHz,} \mathrm{CDCl} 3$ )

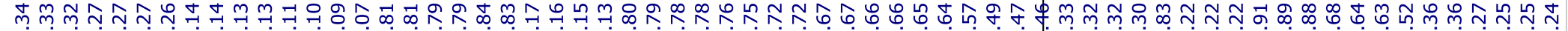

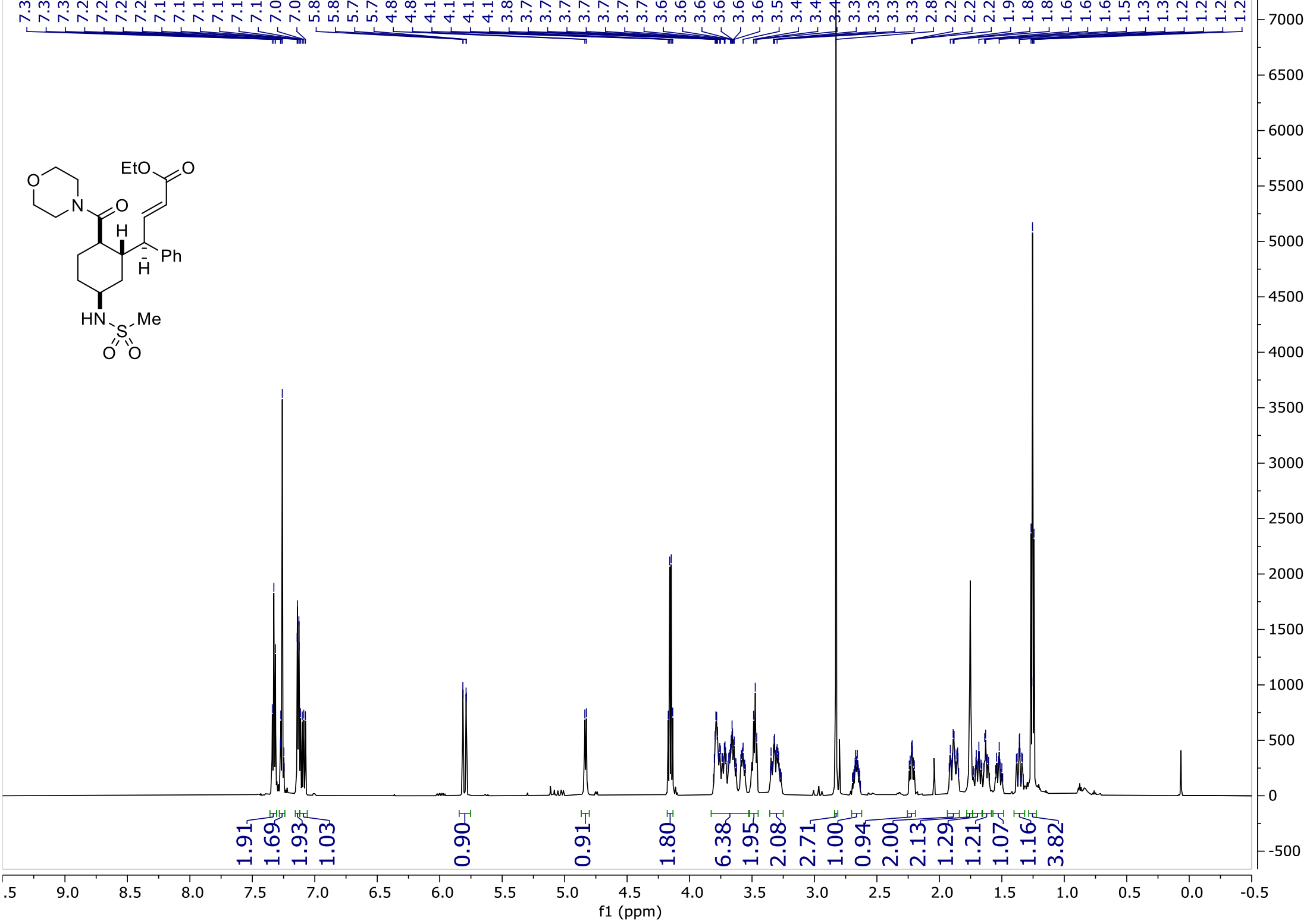

S-136 
ethyl (R,E)-4-((1S,2S,5S)-5-(methylsulfonamido)-2-(morpholine-4-carbonyl)cyclohexyl)-4-phenylbut-2-enoate (10) $\left.{ }^{13} \mathrm{C} \mathrm{Spectrum} \mathrm{(151} \mathrm{MHz,} \mathrm{CDCl} 3\right)$

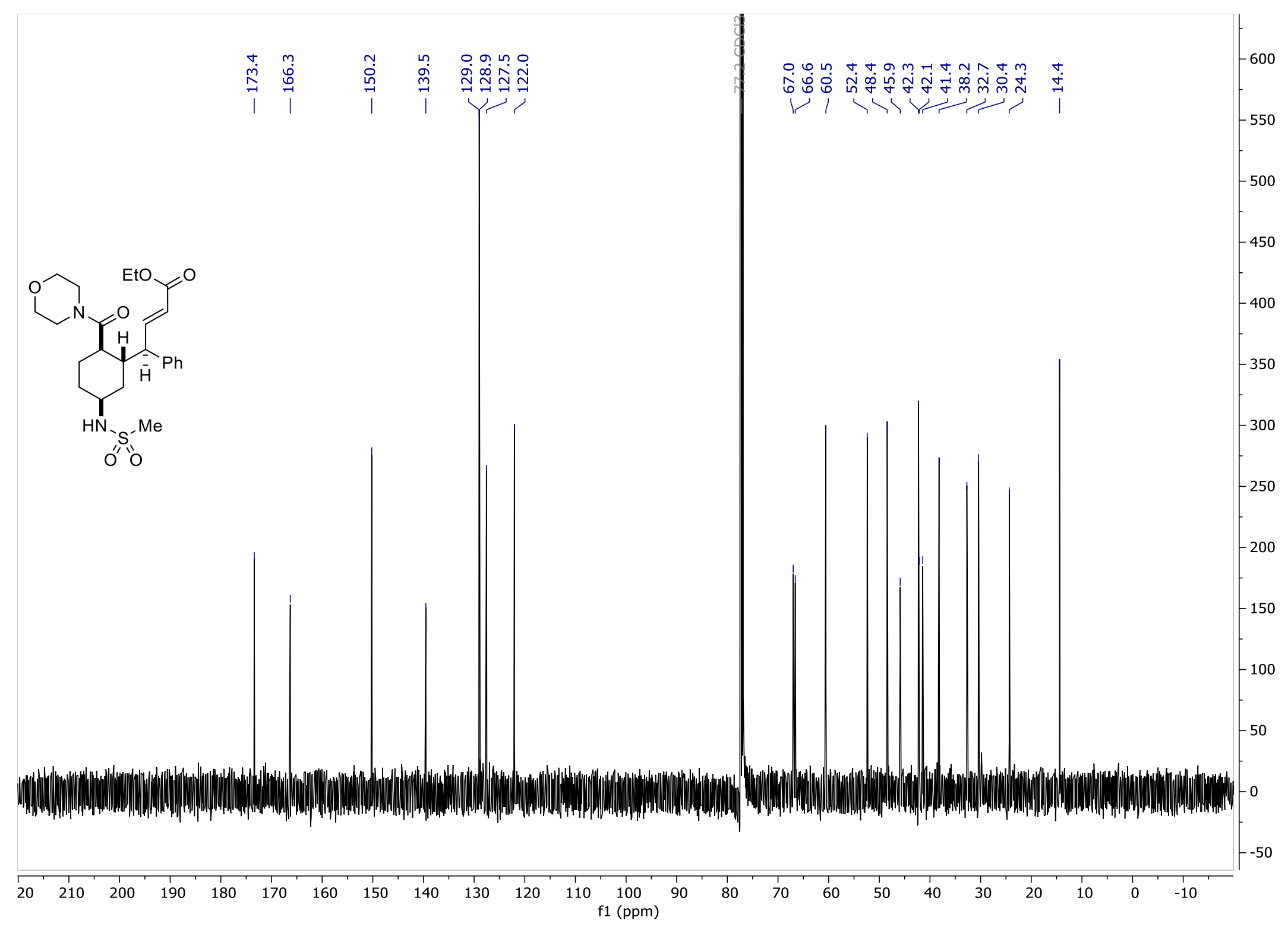

